Growth monitoring during pulsed laser deposition

\title{
of oxides using
}

atomic force microscopy

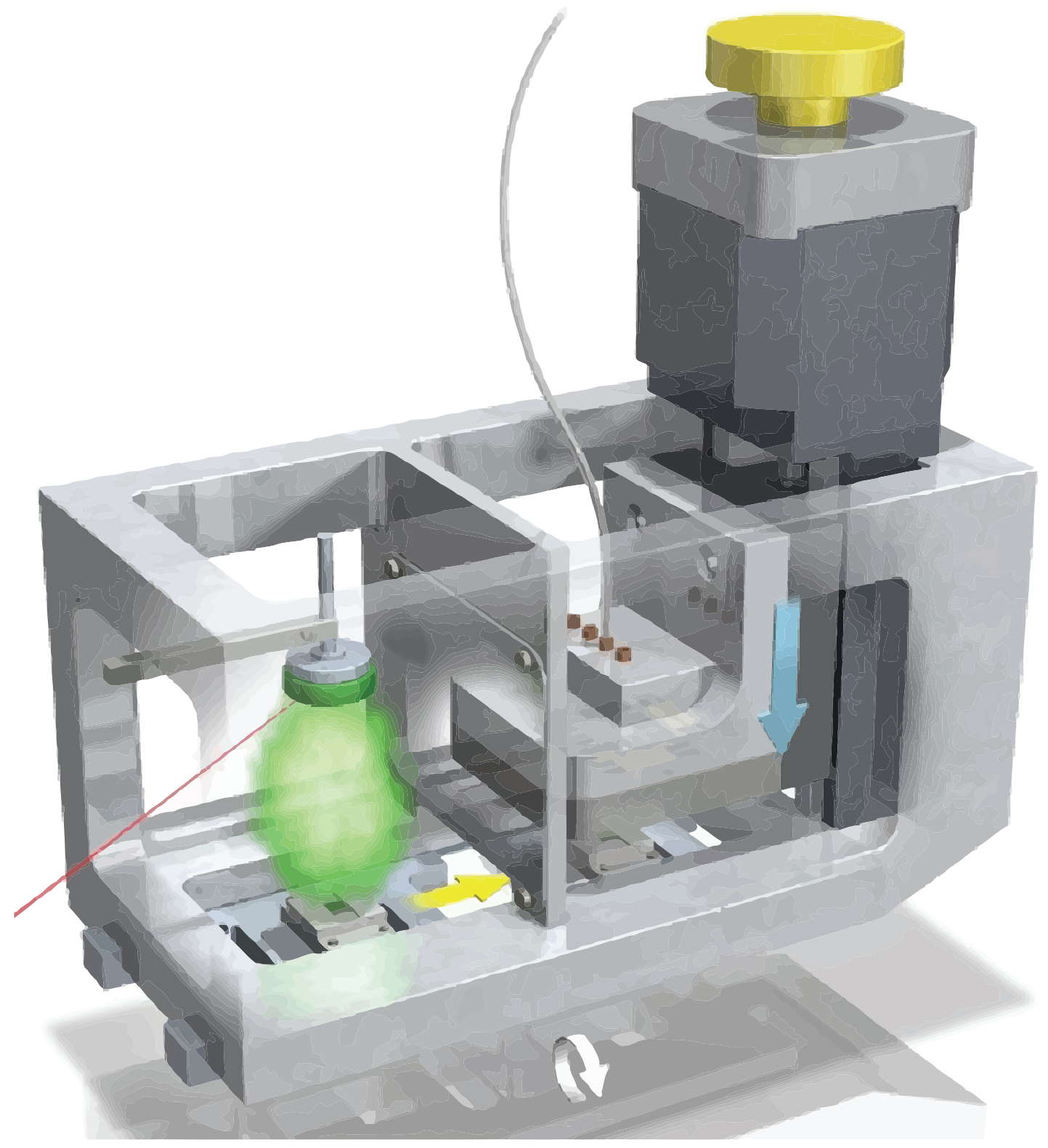

Werner Wessels 
Growth monitoring during pulsed laser deposition of oxides using atomic force microscopy 
Cover: Illustration of developed experimental setup.

\section{Ph.D. committee}

\section{Chairman and Secretary}

Prof. dr. ir. J.W.M. Hilgenkamp (University of Twente)

\section{Promotor}

Prof. dr. ing. A.J.H.M. Rijnders (University of Twente)

\section{Co-promotor}

Prof. dr. ir. G. Koster (University of Twente)

\section{Members}

Prof. dr. ir. B.J. Kooi (University of Groningen)

Prof. dr. J. Aarts (University of Leiden)

Prof. dr. ir. H.J.W. Zandvliet (University of Twente)

Prof. dr. ing. D.H.A. Blank (University of Twente)

\section{Referee}

Dr. G.J.C. van Baarle (Leiden Probe Microscopy B.V.)

The research described in this thesis was carried out within the Inorganic Materials Science group, Department of Science and Technology and the MESA+ institute for Nanotechnology at the University of Twente. This thesis is part of NanoNextNL, a micro and nanotechnology innovation consortium of the Government of the Netherlands and 130 partners from academia and industry. More information on www.nanonextnl.nl.

Growth monitoring during PLD of oxides using AFM

Ph.D. thesis, University of Twente, Enschede, The Netherlands

ISBN: 978-90-365-4219-7

DOI: 10.3990/1.9789036542197

Printed by: Gildeprint Drukkerijen, Enschede, The Netherlands

Author email: diekink@hotmail.com

(C) Werner Wessels, 2016 


\title{
GROWTH MONITORING DURING PULSED LASER DEPOSITION OF OXIDES USING ATOMIC FORCE MICROSCOPY
}

\section{ProefsChRIFT}

\author{
ter verkrijging van \\ de graad van doctor aan de Universiteit Twente, \\ op gezag van de rector magnificus, \\ Prof. dr. T.T.M. Palstra \\ volgens besluit van het College voor Promoties \\ in het openbaar te verdedigen \\ op vrijdag 9 december 2016 om 12:45 uur
}

door

Werner Alexander Wessels

Geboren op 4 december 1986

te Markelo 
Dit proefschrift is goedgekeurd door de promotor

Prof. dr. ing. A.J.H.M. Rijnders

en de co-promotor

Prof. dr. ir. G. Koster 


\section{Contents}

I Background \& Motivation 1

1 Growth monitoring during PLD of oxides using AFM 3

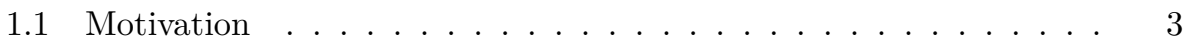

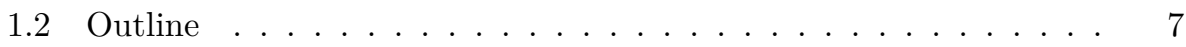

2 Growth monitoring during PVD of oxides 9

2.1 Introduction . . . . . . . . . . . . . . . . 9

2.2 Oxides . . . . . . . . . . . . . . . . . . 11

2.3 Physical vapor deposition . . . . . . . . . . . . . . . 14

2.4 Nucleation and growth . . . . . . . . . . . . . . . 19

2.5 Growth monitoring using diagnostic tools . . . . . . . . . . . 21

2.6 Kinetic Monte Carlo simulations . . . . . . . . . . . . . . . 25

2.7 AFM monitoring of growth kinetics during PLD . . . . . . . . . 27

2.8 Conclusions ......................... 42

II Instrumentation \& Methods 43

3 The development of an AFM to study oxide growth during PLD 45

3.1 Introduction . . . . . . . . . . . . . . . 46

3.2 AFM specifications . . . . . . . . . . . . . . . . . 48

3.3 Concept .......................... 50

3.4 Design .............................. 51

3.5 Performance...................... 62

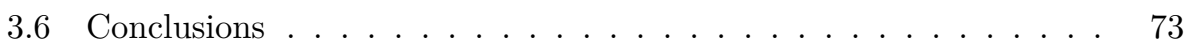

4 Fast side approach for AFM $\quad \mathbf{7 5}$

4.1 Introduction . . . . . . . . . . . . . . . . . 76

4.2 Specifications needed for AFM monitoring of PLD growth . . . . . 77

4.3 Side approach concept . . . . . . . . . . . . . 80 
4.4 Design ......................... 82

4.5 Performance...................... 84

4.6 Conclusions ......................... 92

III Experiments \& Models 93

5 Energetics of vicinal perovskite oxide surfaces $\quad 95$

5.1 Introduction . . . . . . . . . . . . . . . 96

5.2 Perovskite oxide surface energetics . . . . . . . . . . . . . . 98

5.3 Experiment . . . . . . . . . . . . . . . . . . . 102

5.4 Methods . . . . . . . . . . . . . . . . . . 104

5.5 Experimental results \& discussion . . . . . . . . . . . . . 105

5.6 Conclusions \& outlook . . . . . . . . . . . . . . . . . . . . . . 115

6 Sticking of volatile species in perovskite oxides 117

6.1 Introduction . . . . . . . . . . . . . . . . . 118

6.2 Sticking coefficient in perovskite oxides . . . . . . . . . . . . . . . . 119

6.3 Experiment . . . . . . . . . . . . . . . . 121

6.4 Methods . . . . . . . . . . . . . . . . . . 123

6.5 Kinetic Monte Carlo simulations . . . . . . . . . . . . . . . . 125

6.6 Experimental results . . . . . . . . . . . . . . . . . . 131

6.7 Discussion . . . . . . . . . . . . . . . . . . 136

6.8 Conclusions \& outlook . . . . . . . . . . . . . . . . . 138

$\begin{array}{ll}\text { Bibliography } & 141\end{array}$

$\begin{array}{ll}\text { Summary } & 162\end{array}$

$\begin{array}{ll}\text { Samenvatting } & 166\end{array}$

$\begin{array}{ll}\text { List of Publications } & 168\end{array}$

$\begin{array}{ll}\text { Curriculum Vitae } & 170\end{array}$ 


\section{Part I}

\section{Background \& Motivation}





\title{
Chapter 1
}

\section{Growth monitoring during pulsed laser deposition of oxides using atomic force microscopy}

\begin{abstract}
This chapter gives an introduction to growth monitoring during pulsed laser deposition (PLD) of oxides using atomic force microscopy (AFM). In material science, the growthstructure-property relation is studied. Understanding growth gives access to control and manipulation of material properties. Here, examples of diagnostic tools and studies regarding PLD oxide film growth are described. From there on, a motivation is given how in situ atomic force microscopy can have a major contribution to a deeper understanding of oxide thin film growth by PLD. This chapter ends by presenting the outline of this thesis.
\end{abstract}

\subsection{Motivation}

One of the meanings of growth is "an increase in size over a certain time of period", while monitoring means "observing, checking, or keeping a continuous record of a systems state" [1]. So, growth monitoring can be translated as the observation or continuous record of a size increase in a system over a certain time of period. 
From a materials science point of view, growth monitoring is described as the observation of the growth front of a material system. Many material scientists study artificially fabricated materials with reduced dimensions, such as thin films. Understanding and manipulating thin film growth down to the (sub)nanometer length scale enables the controlled fabrication of novel materials containing new atomic arrangements and accompanying functionalities.

Thin films are material layers with a thickness ranging from (sub)nanometer to several micrometers. These films can be grown on substrates using various deposition methods. In physical vapor deposition (PVD) techniques, material is typically evaporated from a target and subsequently deposited on a substrate surface. In general, nucleation and growth take place on the surface during deposition [2]. Nucleation is the stage where adatoms form islands on the surface. Once a critical nucleation density/size is reached, adatoms diffuse on or in between islands to form new nuclei centers or adatoms become attached to island edges. Understanding of nucleation and growth and the growth front gives access to atomic growth control of thin films.

A fascinating material class are the perovskite oxides due to their wealth in available physical properties, such as superconductivity, ferromagnetism, ferro- and dielectricity [3]. Perovskite oxides, with the $\mathrm{ABO}_{3}$ crystal structure, can be fabricated with a wide variety of $\mathrm{A}$ and $\mathrm{B}$ elements. The $\mathrm{B}$ cation is surrounded by a six-fold coordinated oxygen octahedra, where the latter is the main building block of this crystal structure. This material class, with similar lattice constants but various physical properties, is suited for fabrication of heteroepitaxial materials [4]. Heteroepitaxy refers to the preferred crystal structure ordering of a different film material with the underlying substrate.

From 1987 on, pulsed laser deposition (PLD) has become a popular PVD thin film growth technique to fabricate high quality (perovskite) oxide materials. The interest in PLD was induced by the discovery of high- $T_{\mathrm{C}}$ superconductors grown by PLD $[5,6]$. This technique is unique compared to other PVD techniques, such as molecular beam epitaxy (MBE) and magnetron sputtering due to the pulsed nature of flux [7]. Especially this feature has been used throughout this thesis.

Among the current in situ diagnostic tools, reflection high-energy electron diffraction (RHEED) is by far the most commonly applied technique for monitoring in operando the oxide thin film growth. The PVD growth of oxides was monitored by RHEED only during (laser-)MBE up to the end at the nineties [9].

"I could not stop looking at the images. It was like entering a new world. This appeared to me as the unsurpassable highlight of my scientific career. Quote Nobel Prize winner Gerd Binnig (one of the inventors of the atomic force microscope), December 1986. 


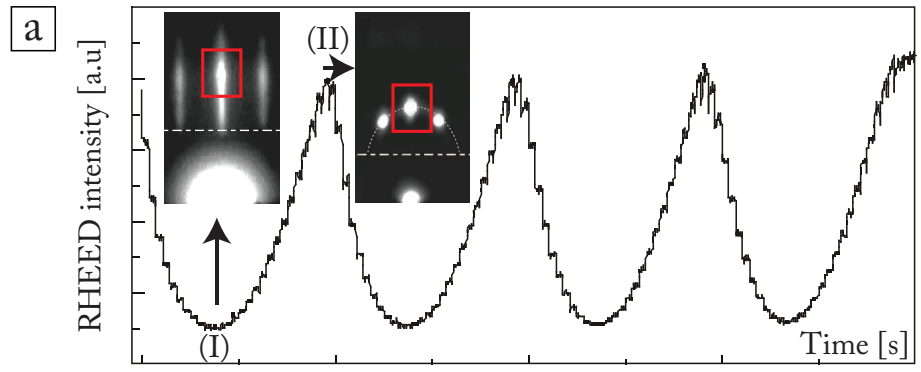

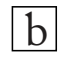

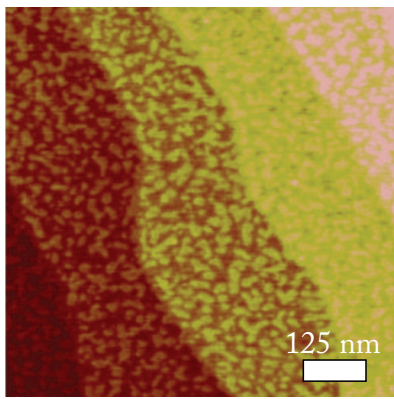

(I)

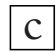

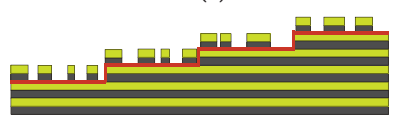

$\mathrm{AO}$

(I) $\square \mathrm{BO}_{2}$

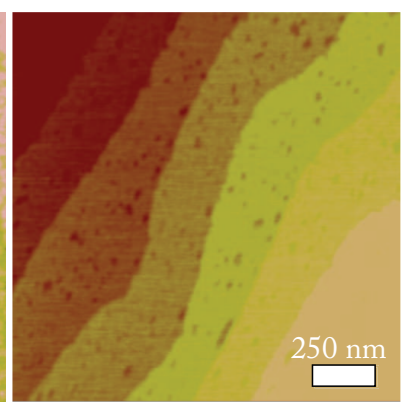

(II)

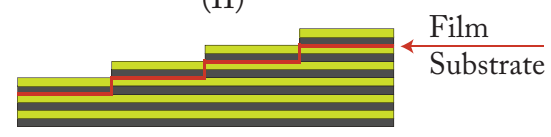

$\mathrm{AO} \quad(\mathrm{II}) \quad \square \mathrm{BO}_{2}$

Fig. 1.1 Growth monitoring of perovskite oxides $\left(\mathrm{ABO}_{3}\right)$ thin films deposited by PLD, a) RHEED specular spot (spot surrounded by red box) intensity monitoring during 2D / layer-by-layer growth with in the inset the corresponding RHEED diffraction pattern, b) typical ex situ AFM images of the surface topography after deposition is stopped at the position of the indicating arrow, c) illustration of the $\mathrm{ABO}_{3}$ surface at the (I) minimum and (II) maximum of a RHEED oscillation. In situ AFM during PLD enables direct measurement of the growth kinetics by monitoring the growth front. Fig. included with permission from Rijnders [8].

At that time, RHEED could only be utilized during oxide deposition at (ultra) high vacuum (UHV) as RHEED is hampered by electron scattering at high oxygen background pressures. In the same period, PLD systems were modified such that they could operate at UHV, sometimes referred to as laser-MBE, enabling RHEED monitoring. Furthermore, low pressures of molecular oxygen [10, 11], $\mathrm{NO}_{2}[12,13], \mathrm{O}_{3}[14]$ and pulsed oxygen sources [15] were applied to incorporate oxygen in the growing film, while being able to monitor growth using a charge particle beam. However, in practice best perovskite oxide PLD growth was obtained at high oxygen background pressure, and therefore a diagnostic tool was required operating at this pressure condition. In 1997, Rijnders et. al designed a 
high-pressure RHEED-PLD system capable of operating up to pressures of $50 \mathrm{~Pa}$ using differential pumping [16]. They were able to monitor the growth of single perovskite oxide unit cell layers [17, 18]. Nowadays, more PLD systems become equipped with RHEED for in situ growth monitoring.

The use of high pressure RHEED-PLD systems led to many interesting observations in the field of perovskite oxides. It has for example been shown that an atomically flat single terminated substrate is a requirement for atomic growth control $[19,20]$. Next to that, Lee et al. found polarization enhancement by growing a superlattice of $\mathrm{BaTiO}_{3}, \mathrm{SrTiO}_{3}$ and $\mathrm{CaTiO}_{3}$, each of two unit cells thick, in a controlled manner [21]. Surprisingly, an extreme carrier mobility was observed at the $\mathrm{SrTiO}_{3} / \mathrm{LaAlO}_{3}$ interface, two wide band gap insulators, dependent on the exact atomic stacking at the mentioned interface [22]. These examples are just a small selection of growth studies, where RHEED was used to obtain improved or novel material properties having atomically flat surfaces/interfaces.

Although RHEED has become an established research tool to monitor growth of oxides in operando, it does not allow to measure the growth of local islands. No direct measurement of the local nucleation density and island radius over time is possible, as the surface reflectivity signal is averaged over a large surface area ${ }^{1}[18]$. In order to study growth kinetics, it would be beneficial to acquire locally growth of islands and the nucleation density. This issue can be overcome by real-space surface monitoring, having high spatial resolution in the order of (sub)nanometers. Examples of real-space growth studies have been demonstrated for metals and semiconductors $[23,24,25]$.

Several real-space diagnostic tools are available, each having their own advantages and disadvantages. Scanning probe microscopes (SPM) are the most suitable techniques to monitor growth kinetics as they visualize the surface morphology with (sub)nanometer spatial resolution [26, 27, 28, 29]. SPM enables local monitoring of the growth front over time and therefore growth of islands can be measured [30]. Most perovskite oxides are insulators or have a large band gap, which limits monitoring by utilizing scanning tunneling microscopy (STM), as it is based on a tunneling current flowing between tip and sample [31, 32].

A first design and demonstration of a conventional atomic force microscope (AFM) operating at metal-oxide PLD conditions has been reported [33, 34]. Until recently, the main drawback of AFM was the sample throughput, since real-time monitoring the PLD growth kinetics requires high-speed AFM instrumentation. Conventional AFM's are slow due to the low bandwidth of the cantilever, AFM scanner,

\footnotetext{
${ }^{1}$ RHEED beam $($ size $\approx 250 \mu \mathrm{m})$ under grazing angle of $0.1-5^{\circ}$ results in a beam footprint on the sample surface of a few $\mathrm{mm}$.
} 
AFM electronics and optical detection systems. Many efforts have been made to increase the bandwidth of these components such that the development of highspeed AFM's has been accomplished [35, 36, 37, 38, 39, 40]. These developments could enable monitoring of growth kinetics during PLD using AFM.

In conclusion, RHEED has been mostly applied as a diagnostic tool to monitor oxide thin film growth during PLD. However, in situ AFM during PLD opens the opportunity for local measurement of island growth and nucleation density, which is not possible with RHEED. If real-time AFM monitoring the as-grown surface during PLD can be realized, a massive boost can be given to research on the growth of for example (perovskite) oxides and therefore understanding of heteroepitaxial oxides. Throughout this thesis, a new AFM design, containing a high resonance frequency AFM scanner and high bandwidth SPM electronics, is demonstrated with focus on growth monitoring during PLD of oxides.

\subsection{Outline}

This thesis describes over four years of research and development of an AFM to monitor oxide thin film growth during PLD. The objective is to gain knowledge by real-space observation of initial thin film growth during PLD in order to understand the growth-structure-property relation of (perovskite) oxides in a wider perspective. The described work is subdivided in three parts with each two chapters treating the state-of-the-art on growth monitoring during PVD of oxides, the developed instrumentation and methods and the conducted experimental studies.

In the first part of this thesis, Ch. 1 and 2, the motivation, outline and background of this work are described. A general introduction, motivation and outline are given in Ch. 1. Ch. 2 gives a comprehensive overview of the state-of-the-art on growth monitoring during PVD of oxides. This chapter is mainly devoted to the background on growth monitoring and diagnostic tools, proposed models for nucleation and growth during PVD growth, PVD techniques, such as PLD and MBE, and (perovskite) oxides. Thin film growth during PVD is typically real-time monitored using reciprocal diagnostic tools. However, in situ AFM can monitor thin film growth at the local scale in contrast to reciprocal diagnostic tools.

The second part, which covers Ch. 3 and 4, describes the instrumentation and methods used in this work. In Ch. 3, the developed experimental setup is presented, which is used to carry out this research. Here, an in situ AFM, containing a high resonance frequency scanner and high-speed AFM electronics, is demonstrated. The concept, specifications, design and performance of the instrument are 
presented. It is shown that the developed AFM can operate at temperatures of room temperature $-700{ }^{\circ} \mathrm{C}$ and pressures ranging from $10^{-6}-1 \mathrm{mbar} \mathrm{O}_{2}$, which are typical PLD conditions used for oxide thin film growth. Furthermore, the performance in terms of AFM scan speed is shown and discussed. Ch. 4 proposes a fast tip-sample approach method for AFM. The commonly used tip-sample approach of AFM is a tedious procedure resulting in low sample throughput hiding relevant information in the growth front e.g. the nucleation density and island radius. Here, the side approach method is tested using a faster sample transfer showing a reduced tip-sample approach time. Furthermore, it is demonstrated that the side approach can be applied monitoring the same surface location required for studying local nucleation and growth phenomena.

Thereafter, two experimental studies which are described in Ch. 5 and 6 are presented in the third part of this thesis. Ch. 5 is concerned with a perovskite oxide vicinal surface study to extract several energetic parameters, such as the nearest neighbor energy. The nearest neighbor energy is an important parameter in kinetic Monte Carlo (KMC) simulations. In literature, a wide range of nearest neighbor energy values are used to simulate perovskite oxide thin film growth. A similar nearest neighbor energy is found for perovskite oxides. The determination of the nearest neighbor energy is a step forward in avoiding iterative simulation processes of perovskite oxide thin film growth. The final chapter, Ch. 6 describes a growth study with the aim to increase sticking of volatile species. In both growth experiments and KMC simulations, temperature modulation during pulsed laser deposition (TM-PLD) has been applied as method. The results reveal that the step density modulation during $2 \mathrm{D}$ growth can be significantly increased compared to deposition at constant temperature. The increased modulation in step density should enhance the probability of sticking. In experiments, an increased sticking coefficient is observed for $\mathrm{Pb}$ in $\mathrm{PbTiO}_{3}$ after using TM-PLD. 


\title{
Chapter 2
}

\section{Growth monitoring during physical vapor deposition of oxides}

\begin{abstract}
This chapter gives a comprehensive overview of growth monitoring during physical vapor deposition of oxides. First, the broad spectrum of physical properties of (perovskite) oxides and their reported values are discussed. Then, the basic principles of the physical vapor deposition techniques pulsed laser deposition and molecular beam epitaxy are described. Several examples are shown of diagnostic tools to monitor thin film growth. The chapter continues with the background and theory in literature to describe nucleation and growth during deposition. In the subsequent section, an in situ atomic force microscopy is proposed as an complementary diagnostic tool to study the initial growth of complex oxides during pulsed laser deposition. Thereafter, the development of high-speed atomic force microscopes will be addressed, which enables the opportunity to monitor in real-space oxide growth kinetic processes.
\end{abstract}

\subsection{Introduction}

The word "oxide" refers to an extremely fascinating material class having a wealth of astonishing physical properties [3]. Besides the wide range of oxide properties in bulk, reducing the thickness down to the atomic scale even opens more phenomena, 
such as a $2 \mathrm{D}$ electron gas (2DEG) at the $\mathrm{LaAlO}_{3} / \mathrm{SrTiO}_{3}$ interface [22]. The variety in physical properties potentially can lead to novel and/or replacement of current electronic devices enabling improved and novel applications for energy harvesting, thermal energy conversion, novel sensors etc. [41]. Analogue to the semiconductor industry, epitaxial strain, doping and confined thickness can be applied to oxides [7, 42].

Functional oxide thin films can be fabricated using various PVD technologies including pulsed laser deposition (PLD) [6, 22, 43, 44, 45], reactive-molecular beam epitaxy (MBE) [46, 47, 48, 49], high-pressure [50, 51, 52] and off-axis sputtering $[53,54,55]$. These PVD techniques enable fabrication of high-quality epitaxial oxide films grown with (sub)nanometer/thickness control and superlattices, abrupt interfaces or construction of new oxide phases can be created. Atomically flat surfaces and interfaces are a requirement for optimization of these oxide heterostructures. This requires in situ growth monitoring in order to understand and control the fabrication of novel functional materials $[56,57]$.

Several diagnostic tools have been adapted to PVD techniques for in situ growth monitoring. Reflection high-energy electron diffraction (RHEED) has been utilized in both MBE and PLD to monitor the growth of oxide thin films [9, 16], the particular growth mode and energy activation barrier for diffusion [10, 17, 18, 58]. Other in situ diagnostic tools, such as surface x-ray diffraction (SXRD), are used to monitor the nucleation density, coverage and subsequent growth of islands in real-time i.e. during homoepitaxial $\mathrm{SrTiO}_{3}$ growth $[59,60]$. Both diagnostic tools are limited in their applicability as only monocrystalline materials can be studied. Once the growth is polycrystalline, growth understanding is lacking as the diffraction signal becomes diffusive. Real-space diagnostic tools, such as scanning probe microscopy (SPM), can overcome this issue as growth can be studied independent of the crystalline state.

Atomic force microscopy (AFM) has the advantage that it allows monitoring of critical PLD growth parameters, such as nucleation density and island radius evolution over time, independent of the crystalline state. Broekmaat et al. showed that AFM imaging can be performed at pressures ranging from $10^{-5}-1$ mbar $\mathrm{O}_{2}$ and temperatures of $500-750{ }^{\circ} \mathrm{C}$ [34]. However, typically the maximum AFM acquisition rate is low (in the order of a few min/frame), which makes it unsuitable to follow growing islands. In last decade, a lot of progress has been made in increasing the AFM acquisition rate up to several frames/s in air and liquids [38, 39]. Recent developments in AFM instrumentation enable the possibility to achieve AFM acquisition rates of frames/s under typical metal-oxide PLD conditions $[61,62,63,64,65,66]$. 
In this chapter, the state-of-the-art on growth monitoring during deposition of oxides using PLD and MBE is presented. First, the diversity in oxide properties are described. In the subsequent section, PVD techniques, such as MBE and PLD and their potential to grow films with atomically flat surfaces and interfaces are discussed. Several growth models have been proposed to describe the thin film growth process which will be presented in the next section. Thereafter, in situ reciprocal diagnostic tools are presented, which have been adapted to PVD techniques to enable monitoring and growth control. AFM can overcome the limitations of reciprocal diagnostic tools discussed in the subsequent section.

\subsection{Oxides}

\subsubsection{Metal-Oxides}

Many metal-oxides exist in nature, since the free energy state of most of the metals in the periodic table is lower in an oxidized state. Other metal-oxides are artificially fabricated [67]. Among the metal-oxides, several crystal structures exist, such as rock salt $(\mathrm{MO})$, rutile or fluorite $\left(\mathrm{MO}_{2}\right)$, antifluorite $\left(\mathrm{M}_{2} \mathrm{O}\right)$, corundum $\left(\mathrm{M}_{2} \mathrm{O}_{3}\right)$ and perovskite $\left(\mathrm{ABO}_{3}, \mathrm{~A}\right.$ and $\mathrm{B}$ are usually a rare-earth and transition metal ion, respectively).

Tab. 2.1 gives an overview of properties with their reported value adapted from Ref. [42]. Schlom et al. listed metal-oxides based on exceptional properties in its category. Most of the reported exceptional properties of metal-oxides are found among the perovskite oxide material class, see Tab. 2.1. The wealth in perovskite oxide properties makes them very popular to study. Moreover, the perovskite oxide material class is interesting for applications in next generation devices, such

\begin{tabular}{cccc}
\hline Property & Value & Oxide & References \\
\hline Piezoelectricity & $d_{33}=2500 p C / N$ & PZNO-PTO & {$[68]$} \\
Ferroelectricity & $P_{S}=105 \mu C / \mathrm{cm}^{2}$ & $\mathrm{PbZr}_{0.2} \mathrm{Ti}_{0.8} \mathrm{O}_{3}$ & {$[69]$} \\
Ferromagnetism & $M_{S}=6.9 \mu_{B} / E u$ & $\mathrm{EuO}$ & {$[70]$} \\
Colossal magnetoresistance & $\Delta R / R_{H}>10^{11}$ & $\mathrm{PSCMO}$ & {$[71]$} \\
Metal-insulator transition & $\Delta R / R_{T l o w}>10^{13}$ & $\mathrm{EuO}$ & {$[72]$} \\
Spinpolarization & $P>98 \%$ & $\mathrm{CrO}_{2}$ & {$[73]$} \\
\hline
\end{tabular}

Tab. 2.1 Examples of metal-oxide properties with their reported values adapted from Ref. [42]. Abbreviations: PZNO-PTO $=\mathrm{PbZn}_{1 / 3} \mathrm{Nb}_{2 / 3} \mathrm{O}_{3}-\mathrm{PbTiO}_{3}$ and $\mathrm{PSCMO}=\mathrm{Pr}_{0.7} \mathrm{Sr}_{0.07} \mathrm{Ca}_{0.26} \mathrm{MnO}_{3-x}$. 


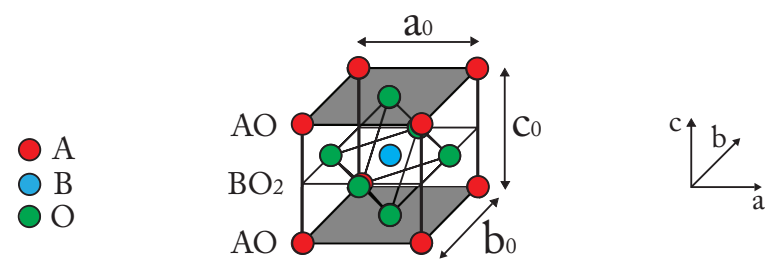

Fig. 2.1 Schematic presentation of the perovskite oxide $\mathrm{ABO}_{3}$ unit cell.

as solar-cells, micro-electromechanical systems (MEMS) and organic light-emitted diodes (OLED's) [7].

\subsubsection{Perovskite oxides}

The perovskite oxide crystal structure is named after Lev Perovski after the discovery of $\mathrm{CaTiO}_{3}$ by Gustav Rose in 1839. The $\mathrm{ABO}_{3}$ crystal structure of perovskite oxides can accommodate around 30 elements and about half of the periodic table on position A and B, respectively [42]. Most perovskite oxide crystal structures are not perfect cubic resulting in a slight distortion of the $\mathrm{BO}_{6}$ octahedra. The oxygen ions are relative large in size compared to the metal cations and the crystal lattice parameters of perovkites are determined by the oxygen backbone. Therefore, the lattice parameters of perovskite oxides are quite similar. In Tab. 2.2, the lattice parameters of many perovskite oxides and perovskite oxide-related phases are shown.

\subsubsection{Perovskite oxide epitaxy}

Epitaxy is a Greek word meaning "in ordered manner" or "arrangement". In thin film deposition, it means the ordering of the crystalline structure of a film with an underlying substrate. Two forms of epitaxy are known, namely homoepitaxy and heteroepitaxy. Homoepitaxy refers to an arranged film on a substrate where both exist of the exact same material, while heteroepitaxy refers to the arrangement of a different film and substrate material. The structural compatibility comes from the fact that they exhibit a small range of in-plane lattice parameters $a_{0}$ ranging between $0.371-0.428 \mathrm{~nm}$, see Tab 2.2, while having a similar $\mathrm{ABO}_{3}$ crystal structure. The similar lattice constants makes perovskite oxides suitable for fabrication of heteroepitaxial structures limiting effects occurring due to large lattice mismatch between film and substrate. Perovskite oxide heterostructures can be fabricated combining properties of different materials or novel properties 


\begin{tabular}{|c|c|c|}
\hline Material & $a_{0}[\mathrm{~nm}]$ & Reference \\
\hline $\mathrm{YAlO}_{3}$ & 0.371 & {$[74]$} \\
\hline $\mathrm{LaSrAlO}_{3}$ & 0.375 & {$[74]$} \\
\hline $\mathrm{LaAlO}_{3}$ & 0.382 & {$[74]$} \\
\hline $\mathrm{CaTiO}_{3}$ & 0.383 & {$[75]$} \\
\hline $\mathrm{Bi}_{2} \mathrm{Sr}_{2} \mathrm{CuO}_{6}$ & 0.383 & {$[76]$} \\
\hline $\mathrm{Bi}_{4} \mathrm{Ti}_{3} \mathrm{O}_{12}$ & 0.384 & {$[77]$} \\
\hline $\mathrm{LaSrGaO}_{4}$ & 0.384 & [78] \\
\hline $\mathrm{YBa}_{2} \mathrm{Cu}_{3} \mathrm{O}_{7}$ & 0.385 & {$[79]$} \\
\hline $\mathrm{NdGaO}_{3}$ & 0.386 & {$[74]$} \\
\hline $\mathrm{LaSrTaO}_{3}$ & 0.387 & {$[74]$} \\
\hline $\mathrm{BiCrO}_{3}$ & 0.390 & {$[80]$} \\
\hline $\mathrm{LaGaO}_{3}$ & 0.390 & [81] \\
\hline $\mathrm{EuTiO}_{3}$ & 0.391 & {$[82]$} \\
\hline $\mathrm{SrTiO}_{3}$ & 0.391 & {$[74]$} \\
\hline $\mathrm{SrBi}_{2} \mathrm{Ta}_{2} \mathrm{O}_{9}$ & 0.391 & {$[83]$} \\
\hline $\mathrm{DyScO}_{3}$ & 0.394 & {$[74]$} \\
\hline $\mathrm{TbScO}_{3}$ & 0.395 & {$[84]$} \\
\hline $\mathrm{BiFeO}_{3}$ & 0.396 & {$[85]$} \\
\hline $\mathrm{GdScO}_{3}$ & 0.396 & {$[74]$} \\
\hline $\mathrm{SmScO}_{3}$ & 0.399 & {$[86]$} \\
\hline $\mathrm{KTaO}_{3}$ & 0.399 & {$[87]$} \\
\hline $\mathrm{BaTiO}_{3}$ & 0.399 & {$[75]$} \\
\hline $\mathrm{NdScO}_{3}$ & 0.401 & {$[86]$} \\
\hline $\operatorname{PbZr}_{x} \mathrm{Ti}_{1-x} \mathrm{O}_{3}$ & 0.404 & [88] \\
\hline $\mathrm{BiScO}_{3}$ & 0.414 & [89] \\
\hline$(\mathrm{Ba}, \mathrm{K}) \mathrm{BiO}_{3}$ & $0.426-0.428$ & {$[90]$} \\
\hline
\end{tabular}

Tab. 2.2 Pseudocubic or pseudotetragonal a-axis lattice parameter $a_{0}$ of different perovskite oxide and perovskite oxide-related phases. 


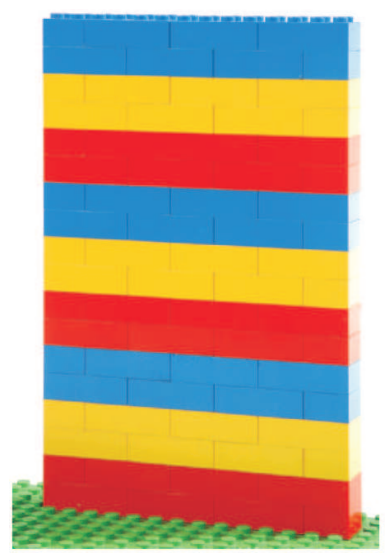

Fig. 2.2 LEGO $^{\mathrm{TM}}$ version representing a superlattice structure. Base represents the substrate. Fig. included with permission from Rijnders and Blank (2005) [4].

appear i.e. at the interface between two materials [22]. As an example, Lee et al. built such perovskite oxide superlattices containing a repetitive structure of two monolayers $\mathrm{BaTiO}_{3}, \mathrm{SrTiO}_{3}$ and $\mathrm{CaTiO}_{3}$ showing a polarization enhancement [21]. Rijnders and Blank (2005) used the analogy of the fabrication of perovskite oxide single layers, heterostructures and superlattices (repetitive structure of several layers) with building LEGO on the atomic scale [4].

\subsection{Physical vapor deposition}

Oxide thin films can be grown using a variety of physical vapor deposition (PVD) techniques. In this section, the two commonly used PVD methods and their characteristic properties to synthesize oxide thin films are shown.

\subsubsection{Physical vapor deposition}

The physical vapor deposition (PVD) method refers to techniques producing a source gas by evaporation, sputtering or other non-chemical methods in a vacuum. In the case of evaporation, the material from the target is transferred into a vapor moving to a substrate surface forming a thin film by condensation. Evaporation is used in several deposition techniques from resistive wire heating to UHV techniques, such as molecular beam epitaxy (MBE) (during evaporation, a target is heated to overcome the binding energy of atoms until they vaporize). The second type of producing a vapor, called sputtering, is the removal of target atoms 
into a vapor source by momentum transfer of accelerating ions from the background gas (background ions are accelerated by applying an electric field). Atoms can be removed only when the energy of accelerating ions is a few times larger than the binding energy of the target atoms. Most oxides are sputtered applying a radio frequency $(\mathrm{RF})$ field reducing surface charging of an electrically insulating material. Laser ablation is quite similar to evaporation, but the energy is supplied by a locally heated laser spot instead of heating the entire material. The PVD technique based on laser ablation is known as pulsed laser deposition (PLD).

Two examples of widely used deposition techniques for epitaxial growth of complex oxide thin films are MBE and $\mathrm{PLD}^{1}$. The conditions in which an oxide thin film is deposited are completely different. In MBE, oxide thin films are deposited at an UHV background pressure range of $10^{-9}-10^{-4}$ mbar, while in PLD oxide thin films are typically deposited at oxygen pressures ranging from $10^{-6}-1$ mbar pure $\mathrm{O}_{2}$. Another main difference between MBE and PLD is the way stoichiometric transfer is achieved. In PLD, the target contains the same stoichiometry as later on obtained in the film, where oxidation takes place by interaction between plasma plume and oxygen background gas. In MBE, a target with a single element is continuously evaporated forming a so-called molecular beam and oxidation takes place at the surface.

\subsubsection{Molecular beam epitaxy}

MBE is a PVD technique in which thermal beams of atoms or molecules react with a surface to form a crystalline film. A single-crystalline substrate is positioned in the center of the MBE vacuum chamber. The substrate is heated to the growth temperature to achieve the desired phase of the thin film. Molecular beams are deposited from different effusion cells. Every molecular beam is evaporated from a single effusion cell as multicomponent mixtures rarely evaporate congruently [42]. Shutters are installed in front of every molecular beam to control the deposition interval. Both the shutter time and temperature are parameters to control the layering of heterostructures. This control enables the growth of layers down to a single unit cell thick.

Elemental species in MBE are oxidized after the species reach the substrate. The long mean free path of species should not be destroyed and therefore the pressure (dependent on the oxide material) during oxide deposition is limited to $P \leq 10^{-4}$ mbar. Molecular oxygen has been applied during MBE in order to easily

\footnotetext{
${ }^{1}$ In essence, MBE and PLD are different, but both PVD techniques are unique as they allow fabrication of thin films controlling the film thickness with the precision of a single monolayer.
} 
oxidize metal species, while higher activity oxidants are used for oxidizing species e.g. $\mathrm{Bi}, \mathrm{Pb}$ and $\mathrm{Cu}[42]$. To prevent inadequate composition control, atomic absorption spectroscopy (AA) has been adapted allowing flux measurements down to an error lower than 1\% [91]. A retractable quartz crystal microbalance provides an absolute in situ flux measurement at the substrate position.

MBE was developed to deposit GaAs and (Al,Ga)As [92]. This PVD technique allows controlled layering of monolayers in combination with its growth diagnostics and fabrication of thin films has been expanded to other classes of materials, such as metals and oxides [93]. Besides molecular beams emanating by heated crucibles of individual elements, molecular beams of gasses are introduced during oxide deposition. This variant of MBE is called reactive MBE [42]. The growth of oxide heterostructures using reactive MBE started around 30 years ago. In 1985, Betts and Pitt reported on epitaxial growth of $\mathrm{LiNbO}_{3}$ films [94]. It turned out that $\mathrm{LiNbO}_{3}$ was more difficult to grow compared to several oxides deposited afterwards [93]. Since the discovery of high temperature superconductivity [5], oxide MBE has been used to grow oxides other than $\mathrm{LiNbO}_{3}$, such as superconductors $[46,47,95,96,97,98]$, ferroelectrics $[49,94,99,100,101,102$, 103], ferromagnets [104, 105, 106, 107, 108, 109], magnetoelectrics [106], multiferroics $[110,111,112,113,114]$ and superlattices $[100,115]$. The first integration of an oxide $\left(\mathrm{SrTiO}_{3}\right)$ on $\mathrm{Si}$ was reported in 1998 [101]. Schlom (pioneer in the oxide MBE field) concluded based on literature that epitaxial $\mathrm{SrTiO}_{3}$ can be grown on Si with a narrow full width at half maximum (FWHM) of $0.008^{\circ}$ in its rocking curve, smaller than $0.068^{\circ}$ achieved by other deposition techniques [93]. It has to be noted that not every oxide is stable on Si [116]. Besides oxide MBE deposition on $\mathrm{Si}(001)[99,107,117]$, oxides have been grown on several other semiconductors, namely $\operatorname{Ge}(001)$ [118], $\operatorname{GaAs}(001)$ [119] and $\operatorname{GaN}(0001)$ [120, 121].

\subsubsection{Pulsed laser deposition}

Before 1986, pulsed laser deposition (PLD) was a rarely used and a somewhat exotic PVD method in thin film laboratories. In the beginning of the sixties, Breech and Cross succeeded to vaporize atoms from a solid surface using a ruby laser. A few years later, Smith and Turner published the first vacuum PLD (using a ruby laser) growth of different solid compounds onto a substrate having stoichiometric transfer [122]. Only a few achievements are reported in the following decades, such as pulsed laser evaporation of powders leading to the thin film growth of $\mathrm{SrTiO}_{3}$ and $\mathrm{BaTiO}_{3}[123]$ and i.e. inter-metallic materials were fabricated using a pulsed laser beam [124]. In 1983, superconductivity in pulsed laser evaporated $\mathrm{BaPb}_{1-\mathrm{x}} \mathrm{BixO}_{3}$ films using a post heat treatment was reported by Zaitsev and their 
co-workers [125]. The breakthrough for PLD came by the discovery of the high$T_{\mathrm{c}}$ (above boiling point nitrogen) superconducting thin films of $\mathrm{LaBaCuO}[5]$ and $\mathrm{YBaCuO}[6]$ in the period $1986-1987^{2}$. This discovery started massive research efforts with focus on growing high- $\mathrm{T}_{c}$ superconducting cuprates and different other complex oxide phases and materials.

In the nineties, PLD became more established and 2D growth was monitored using reflection high-energy electron diffraction (RHEED) at UHV [9]. PLD at UHV is called "laser-MBE", but MBE in this term is questionable as the plasma plume contains ions, electrons and neutrals. After the introduction of high-pressure RHEED in 1997 [16], several studies focused on PLD growth of oxides, such as $\mathrm{SrRuO}_{3}$ [18] and superlattices of $(\mathrm{Ba}, \mathrm{Sr}, \mathrm{Ca}) \mathrm{CuO}_{2}$ [17]. A few years later, Eres et al. presented an in situ time-resolved surface x-ray diffraction (SXRD) study providing direct physical insight beyond RHEED. In 2004, a 2DEG has been found between the interface of $\mathrm{SrTiO}_{3}-\mathrm{LaAlO}_{3}$ grown in a controlled manner using PLD [22]. In this period, production related issues, such as large-area scale-up have been addressed. Nowadays, companies i.e. Twente Solid State Technology (TSST) and SolMates explore the possibilities of integrating oxides on 4 - 8 inch silicon wafers using large-area PLD techniques [7].

The key component of a PLD setup is the pulsed laser. In the field of oxides, mostly an UV laser is used due to the high absorption of laser energy ${ }^{3}$. Material is ablated from the target forming a vapor. Due to the pressure gradient, the vapor called "plasma plume" moves towards the sample surface. Particles with tunable kinetic energies penetrate typically through an oxygen pressure in the range of $10^{-6}-1$ mbar $\mathrm{O}_{2}$. Typical kinetic energies of species during oxide PLD varies in a range of few $\mathrm{eV}$ - several hundred $\mathrm{eV}$ [18]. Particles in the plasma plume interact with i.e. the oxygen molecules in the background gas resulting in oxydation of the ablated species and reduced kinetic energy $E_{\text {kin }}$. Upon arrival of the species at the substrate surface, absorption takes place followed by diffusion and sticking/desorption. The adatom diffusion, absorption and desorption probability are controllable parameters to influence the PLD thin film growth behavior.

PLD has some unique features, namely 1 ) oxidation of species before reaching the substrate, 2) a high deposition rate $R$ and 3) deposition and growth are separated in time $t$. Recently, it has been found that surface diffusion is heavily influenced by oxidation [126]. Within the deposition pulse, deposition rates $R$ of $10^{2}-10^{5} \mathrm{~nm} / \mathrm{s}$ can be achieved, while other PVD techniques have typical deposition rates of

\footnotetext{
${ }^{2}$ High- $T_{\mathrm{c}}$ superconductors are materials that behave as superconductors at temperatures typically higher than the liquid nitrogen temperature $(77 \mathrm{~K})$.

${ }^{3} \mathrm{~A}$ pulsed excimer ( $\mathrm{ArF}, \mathrm{KrF}$ ) laser is commonly used to deposit oxides with a repetition rate of $0.1-100 \mathrm{~Hz}$.
} 


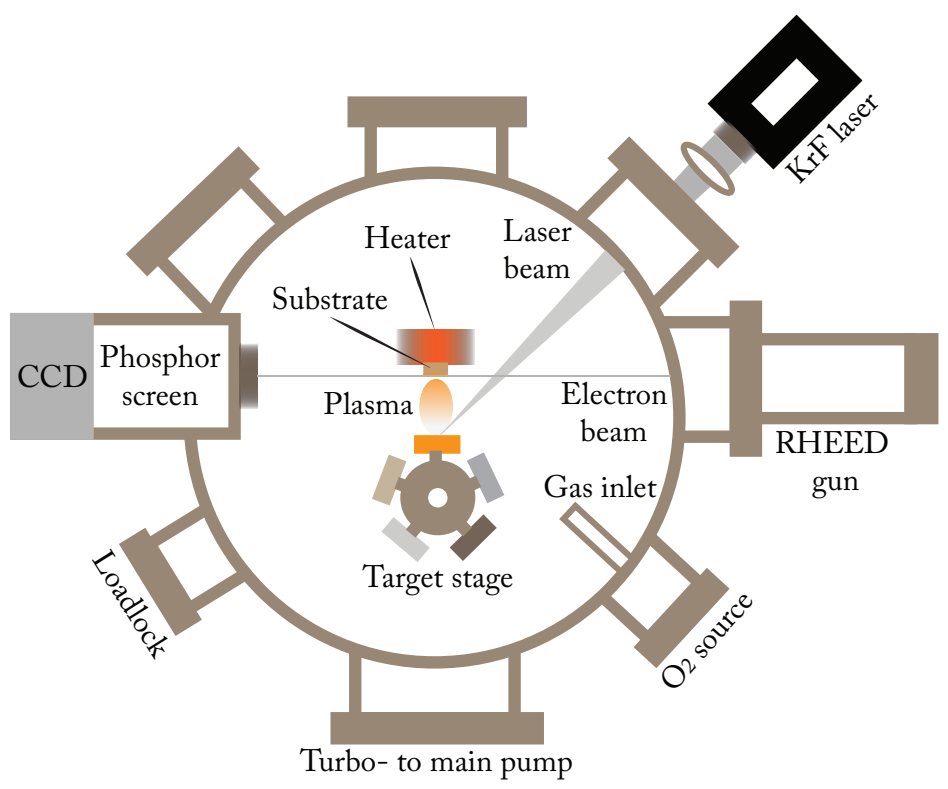

Fig. 2.3 Typical PLD setup in 2016 .

$10^{-2}-10^{-1} \mathrm{~nm} / \mathrm{s}[18]$. The high $R$ of PLD induces a very high degree of the supersaturation $\Delta \mu$, which is given by:

$$
\Delta \mu=k_{\mathrm{B}} T \ln \left(\frac{R}{R_{0}}\right)
$$

where, $k_{\mathrm{B}}$ is the Boltzmann constant, $T$ is the deposition temperature, $R$ is the actual deposition rate and $R_{0}$ the equilibrium rate. This high degree of supersaturation $\Delta \mu$ induces on its turn a high nucleation density with small nucleation radius [2]. In contrast to other PVD techniques, the PLD deposition flux is not constant over time causing separation of deposition and growth of islands in time $t$. The majority of the studies argue that this is the case [7, 18, 44], with the exception of Christen and Eres [127].

Nowadays, a typical PLD setup looks like the schematic illustration in Fig. 2.3. For fabrication of oxide thin films, a $\mathrm{KrF}$ excimer laser is typically utilized with a pulse energy of several hundreds of $\mathrm{mJ}$ and a pulse width of $25 \mathrm{~ns}$. A mask is placed in the optical beam to select only a homogeneous part of the laser beam. Mostly, the laser beam (guided by an optical focal lens) is focused at an angle of $45^{\circ}$ on the target. The energy density of the laser beam is adjustable by the mask size, demagnification and laser energy. A target holder capable of holding several targets and a heater sample holder with heater holder are mounted both on a computer controlled XYZ rotational stage (the heater holder is guided through 
a loadlock without breaking the vacuum level ranging from $10^{-8}-10^{-6}$ mbar for different PLD systems.

\subsection{Nucleation and growth}

In this section, the general theory behind nucleation and growth of oxide thin films during PVD techniques, such as MBE and PLD is described.

\subsubsection{Thermodynamic equilibrium}

In thermodynamic equilibrium, three growth modes are known describing crystal growth, namely Frank-van der Merwe growth [128], Volmer-Weber growth [2] and Stranski-Krastanov growth [2]. The different growth modes are explained by the surface energies of the film $\left(\gamma_{\mathrm{F}}\right)$ and substrate $\left(\gamma_{\mathrm{S}}\right)$ and interfacial tension between film and substrate $\left(\gamma_{\mathrm{I}}\right)$. Frank-van der Merwe growth occurs when a film wets the substrate $\left(\gamma_{\mathrm{S}} \geq \gamma_{\mathrm{F}}+\gamma_{\mathrm{I}}\right)$ and the preferred growth direction is in-plane. In the Volmer-Weber growth mode, the film does not wet on the substrate $\left(\gamma_{\mathrm{S}}<\gamma_{\mathrm{F}}+\gamma_{\mathrm{I}}\right)$. Thermodynamic factors, such as misfit strain can induce a growth mode transition from Frank-van der Merwe to Volmer-Weber, so-called Stranski-Krastanov growth mode. At the critical thickness of the film, the misfit strain and interfacial energy become too large resulting in defect formation and change in growth mode.

\subsubsection{Growth kinetics}

The growth front evolution of thin films during PVD, such as MBE and PLD is assumed to be dominated by growth kinetics (instead of thermodynamics) [127]. For example, homoepitaxial PLD growth experiments revealed that kinetic parameters determine the growth mode [17]. The interlayer mass transport is limited by the adatom diffusion length $l_{\mathrm{D}}$ on the surface and/or kinetic step energy barrier. Due to a limited $l_{\mathrm{D}}$, roughening of the surface occurs in practice after the deposition of a monolayer (ML) (therefore, damping of the maximum RHEED intensity is observed after every subsequent grown ML, see Fig. 2.5).

The diffusion length $l_{\mathrm{D}}$ scales with diffusion time $\tau_{\mathrm{D}}$ and the diffusion coefficient $D_{\mathrm{S}}$ according to $l_{\mathrm{D}}=\sqrt{D_{\mathrm{S}} \tau_{\mathrm{D}}}$, where $D_{\mathrm{S}}$ is given by:

$$
D_{\mathrm{S}}=D_{0} e^{\frac{E_{\mathrm{A}}}{k_{\mathrm{B}} T}}
$$


The adatom diffusion length $l_{\mathrm{D}}$ depends on both temperature $T$ and the activation energy barrier for diffusion $E_{\mathrm{A}}$. Depending on the nucleation density/island radii and diffusion length $l_{\mathrm{D}}$, several growth modes exist (see Figs. 2.4 and 2.5), which are described below.

\section{D / Step-flow growth}

The 1D (step-flow) growth mode only occurs on vicinal (oxide) surfaces. Typically, oxide surfaces have a certain miscut angle containing unit cell height steps with a characteristic average terrace width $\langle L\rangle$ between the surface steps, see Ch. 5 . If $l_{\mathrm{D}} \geq\langle L\rangle$, adatoms diffuse towards step-edges and attach to it. Therefore, adatoms nucleate at step-edges and limited nucleation on terraces takes place or nucleation centers are absent. As a result, steps will effectively move [127].

Due to the spread in kinetic energy of arriving species in PLD (the spread is small in $\mathrm{MBE}$ ), an effective $\left\langle D_{\mathrm{S}}\right\rangle$ has to be considered [127]. In a different approach, the effective $\left\langle D_{\mathrm{S}}>\right.$ was used as parameter to predict whether $1 \mathrm{D}$ growth can be expected. This type of growth is expected when:

$$
F<2 N_{\mathrm{p}}<D_{\mathrm{S}}>/<L>^{2}
$$

assuming that the time between laser pulses is larger than the diffusion time $\tau_{\mathrm{D}}$, where $F$ is the average deposition flux per pulse, $N_{\mathrm{p}}$ is the amount of material per pulse [129]. From this it can be concluded, that the existence of $1 \mathrm{D}$ growth depends on average adatom diffusivity, deposition rate and the miscut of the substrate [18]. For MBE, the expression is somewhat different, namely $F<2 D_{\mathrm{S}} a_{0}^{2} /<L>^{4}$, where $F$ has the unit monolayer per second and $a_{0}$ is the in-plane lattice constant [129]. As an example, Rijnders et al. showed that $\mathrm{SrRuO}_{3}$ grows $1 \mathrm{D}$ after a few monolayers deposited on $\mathrm{SrTiO}_{3}(001)$ using PLD [8].

\section{D / layer-by-layer growth}

The 2D (layer-by-layer) growth mode differs from 1D as $l_{\mathrm{D}}<<L>$ and therefore nucleation will take place on a terrace/surface. Nucleation takes place when atoms meet and form clusters on a surface. The characteristic distance between the nucleation of islands is here defined as $\rho$. This value depends on both the flux $F$ and diffusivity coefficient of adatoms $D_{\mathrm{S}}[127]$. After nucleation, islands grow in the period $2 r_{\text {island }}<\rho$, where $2 r_{\text {island }}$ is the island diameter. Under the condition $2 r_{\text {island }} \approx \rho$, islands coalescence and then second layer nucleation takes place. This 


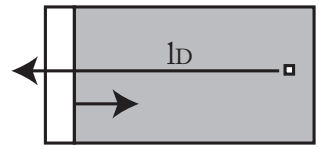

$1 \mathrm{D}$

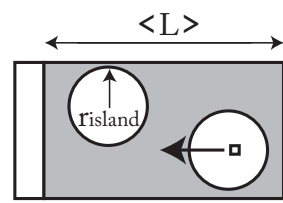

$2 \mathrm{D}$

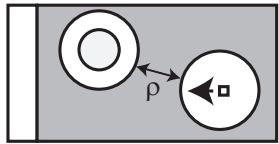

$3 \mathrm{D}$

Fig. 2.4 Schematic illustration of kinetic growth modes.

description represents a perfect $2 \mathrm{D}$ growth of thin films. However, perfect layer-bylayer growth will not be observed in experiments [130]. $\mathrm{SrTiO}_{3}$ homoepitaxy is an example of a model system growing 2D in a wide range of PVD settings $[17,131]$.

\section{D / island growth}

In the multilevel (island growth) growth mode, the diffusion length $l_{\mathrm{D}}<<\rho$ and/or kinetic step energy barrier is too high such that 3D islands are formed at the surface. Here, second layer nucleation starts far before islands coalescence as $l_{\mathrm{D}}$ is small. As a consequence, $l_{\mathrm{D}}$ is rapidly smaller than the island radius $r_{\text {island }}$ and results in limited interlayer mass transport. The probability for second layer nucleation increases rapidly and leads to multiple level growth [127]. In practice, this growth mode will always be in between the described extreme $2 \mathrm{D}$ or $3 \mathrm{D}$ growth modes [18].

\subsection{Growth monitoring using diagnostic tools}

In this section, the most commonly used growth monitoring tools during PVD are described. Many PVD techniques are equipped with reflection high-energy electron diffraction or surface x-ray diffraction in order to monitor oxide growth kinetics.

\subsubsection{RHEED}

Reflection high-energy electron diffraction (RHEED) is a reciprocal diagnostic tool requiring minimal hardware. It is utilized in the field of surface science because of its high surface sensitivity under grazing angles. A high-energy electron beam $(10-50 \mathrm{keV})$ is focused on a surface under a grazing angle of only $0.1-5^{\circ}$. Due to the grazing incidence angle, electrons interact with the topmost layer of only 1 - $2 \mathrm{~nm}$ at the surface (therefore it's surface sensitive). On a phosphorus screen, 
scattered electrons are gathered showing a diffraction pattern characteristic for the crystal structure and containing information about the surface morphology.

Often RHEED is utilized as it allows monitoring of the sample surface during a deposition experiment and operates at a wide range of background pressures (UHV - $50 \mathrm{~Pa}$ ) [16, 56]. This diagnostic tool is popular as in combination with PVD techniques intensity oscillations can be observed of the specular intensity during deposition indicative for a layer-by-layer growth front [56]. Material deposited on an atomically flat surface leads to roughening and a decrease in RHEED specular intensity (see Fig. 2.5), while up to the completion of full layer the surface becomes smoother and therefore the RHEED specular intensity increases all influenced by nucleation, growth and coalescence of islands. RHEED can be used as a tool to monitor the film thickness during 2D growth as an oscillation period corresponds to the growth of a single crystal layer. Generally, it is assumed that the maximum of RHEED specular intensity oscillations refers to a completely grown monolayer.

A model to explain the oscillations in RHEED intensity is the step density model. For both MBE and PLD, the step density model is applied to describe the relation between the specular intensity and density of steps on the surface. The idea behind the model is that every step acts as a diffusive scatterer and therefore the RHEED intensity decreases. Stoyanov and Michailov assumed that the nucleation of islands takes place at $t=t_{\mathrm{ML}}$ (the period for one unit-cell layer coverage), then the step density $S(\mathrm{t})$ as function of coverage $\theta$ is described by [132]:

$$
S(t)=2 \sqrt{\pi N_{0}}(1-\theta) \sqrt{-\ln (1-\theta)}
$$

where $N_{0}$ is the initial number of nuclei at $t=t_{\mathrm{ML}}$. In $2 \mathrm{D}$ growth during PLD, $\theta \propto t$ and a maximum $S$ is measured at $\theta=0.39$, while at constant nucleation and step propagation a maximum $S$ is expected at $\theta=0.5$ [18]. The RHEED intensity $I_{\text {RHEED }}$ is approximately given by:

$$
I_{\text {RHEED }} \propto 1-\frac{S(t)}{S_{\max }}
$$

where $S_{\max }$ is the maximum step density. During PLD, not only surface steps contribute to $I_{\text {RHEED }}$. The relaxation behavior in the RHEED intensity after a deposition pulse can be described by the diffusive atoms, which act as individual scatters [18]. Several studies used this approach to study kinetic factors important in both 2D and 1D growth [8, 17, 18, 44, 58]. These studies evaluated relaxation times from RHEED specular intensity fitting between deposition pulses 


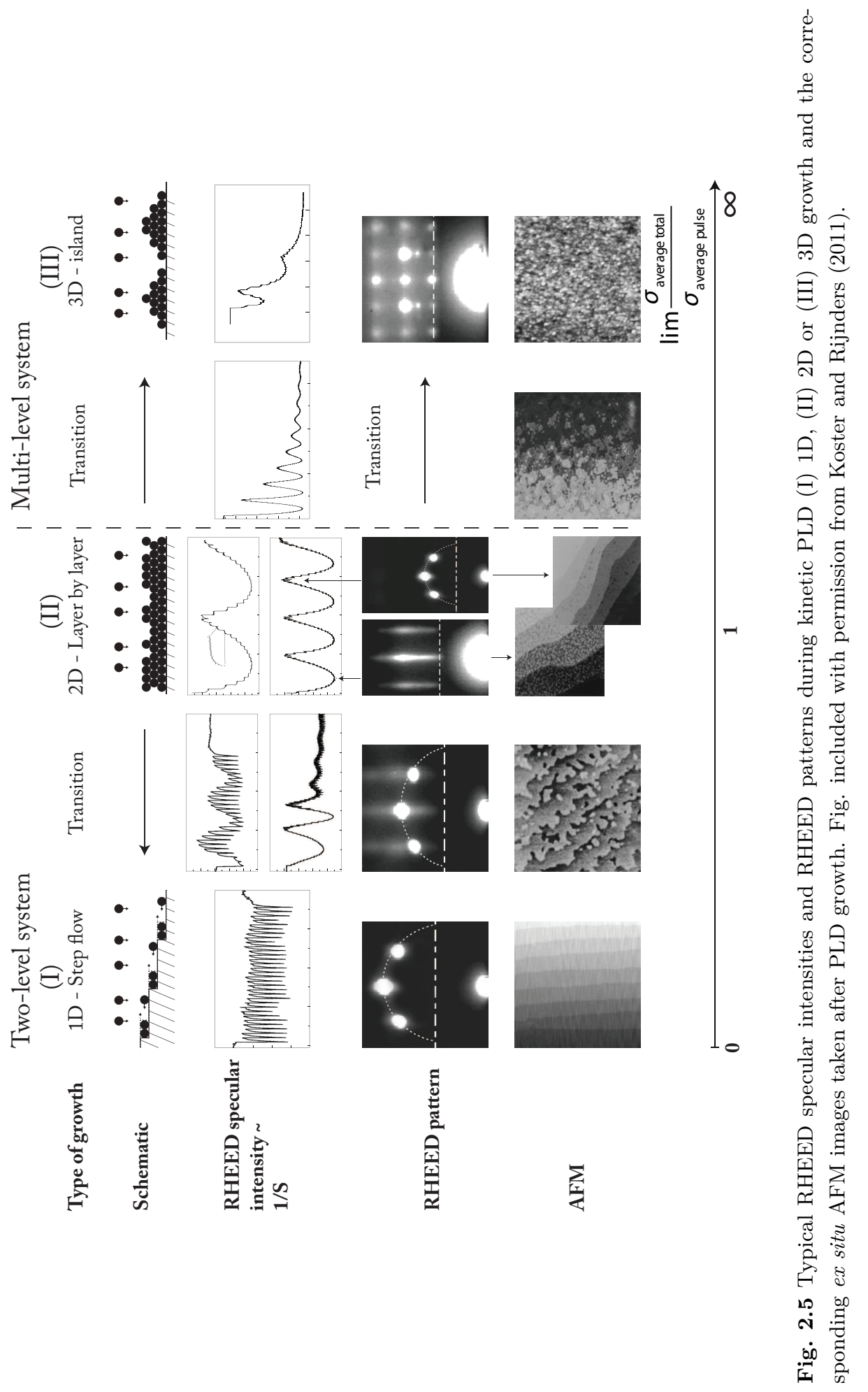


as a function of coverage, temperature and deposition pressure. Assuming 2D growth, relaxation times are extracted by using the function in Eq. 2.6:

$$
I_{\mathrm{RHEED}} \approx I_{0}\left[1-e^{-\frac{t}{\tau}}\right]
$$

where $\tau$ is the extracted relaxation time. A more detailed description and examples can be found in Refs. [8, 17, 18, 44, 58].

RHEED has been utilized in PVD techniques, such as PLD [16], MBE [56] and (RF) sputtering [133] and is often used to monitor the type of growth mode and number of oxide grown layers during $2 \mathrm{D}$ growth [18, 21, 22, 134, 135]. The surface sensitivity enables monitoring of oxide initial layer growth, but also of subsequent layers $[9,18]$. Moreover, RHEED was used as diagnostic tool to study oxide growth manipulation $[44,58]$, oxide growth kinetics $[10,18]$ and qualitative determination of perovskite oxide surface termination [17].

\subsubsection{SXRD}

The dynamic scattering effects during RHEED, due to strong interaction of electrons with the surface atoms, makes it hard to describe growth kinetics by kinematic theories. Mostly, RHEED is performed to measure the growth of single monolayers, but rarely it is used to qualitative describe growth kinetics. Other sources, such as X-rays are not applicable in surface studies due to the large penetration depth and low signal intensity. The latter is overcome by the third generation synchrotron as an X-ray intensity of $10^{12}$ photons/s is available nowadays [127]. The kinematic analysis is a motivation to use synchrotron x-ray diffraction as a tool for monitoring thin film growth. In contrast to RHEED, surface x-ray diffraction (SXRD) enables the quantitative measurement of the surface coverage directly from the measured single scattering intensity.

The formula to describe the SXRD scattering intensity is given by:

$$
I_{\mathrm{SXRD}} \propto\left|F_{\mathrm{c}}(\mathrm{HKL})\right|^{2} /\left[4 \cdot \sin ^{2}(\pi \mathrm{L})\right]
$$

where $\mathrm{I}_{\mathrm{SXRD}}$ is the scattering intensity, $F_{\mathrm{c}}$ the structure factor containing all atomic coordinates. The so-called crystal truncation rods (CTR) are the scattering intensity rods described by Eq. 2.7, with $\left|F_{\mathrm{CTR}}(\mathrm{L})\right|^{2}=1 /\left[4 \cdot \sin ^{2}(\pi \mathrm{L})\right]$. CTR are used in measurements providing information about growth kinetics. They are sensitive to both vertical and lateral surface structure. The specular rod intensity 
(vertical) describes the layer filling during growth, where the diffusive scattering (lateral) contains information about the lateral island distribution. More detailed descriptions can be found in reviews by Robinson [136], Vlieg [137] and Saldin and Shneerson [138].

SXRD has been applied in both MBE and PLD in order to monitor real-time growth kinetics during PVD [59, 60, 136, 139]. A typical setup contains a MBE/PLD chamber, where the diffractometer is integrated such that the sample can be positioned regarding to a fixed incident X-ray beam. In some setups, Be windows are installed resulting in exit X-rays with minimal attenuation and scattering. Most setups vary in chamber configuration, the geometry of the diffractometer and detection scheme. SXRD is often adapted as complementary diagnostic tool besides RHEED [140].

A few SXRD studies have been performed to extend the understanding about the pulsed nature of PLD. The general accepted picture of PLD is the separation between growth and deposition in time $t$. However, there is still some controversy in literature as SXRD studies suggest that both crystallization and most of the interlayer transport occur in the $\mu$ s time scale during deposition instead of directly after deposition $[141,142,143]$. Sullivan et al. showed that the maximum RHEED intensity does not match with the maximum coverage of a single monolayer during $\mathrm{SrTiO}_{3}$ homoepitaxial PLD growth [140]. Besides that, Larsson et al. chopped continuous molecular beams to study the influence of flux modulation on the growth [144]. The effect of flux modulation on the growth during MBE is minimal suggesting that a pulsed thermal beam is not the most important requirement for producing smoother films.

\subsection{Kinetic Monte Carlo simulations}

Weeks and Gilmer introduced a solid-on-solid (SOS) model, which has been frequently utilized to predict thin film growth [145]. The mobility of adatoms on surfaces is often described by SOS based kinetic Monte Carlo (KMC) type models based on activated processes i.e. deposition, evaporation and diffusion ${ }^{4}$. In the KMC model, adatom diffusion is simulated by simultaneously calculating the hopping rates $\left(k_{\text {hopping }}\right)$ for all adatoms on a simulated two-dimensional grid or lattice. Adatoms are only allowed to diffuse in-plane in the KMC model. In thin film growth it is quite reliable due to the $2 \mathrm{D}$ structure of thin films. In the KMC simulations, perfect sticking is assumed resulting i.e. in no re-evaporation.

\footnotetext{
${ }^{4}$ For perovskite oxides, it is assumed that $\mathrm{ABO}_{3}$ unit cells are deposited and diffuse over a simple cubic crystal lattice with the grid size of an $\mathrm{ABO}_{3}$ unit cell.
} 


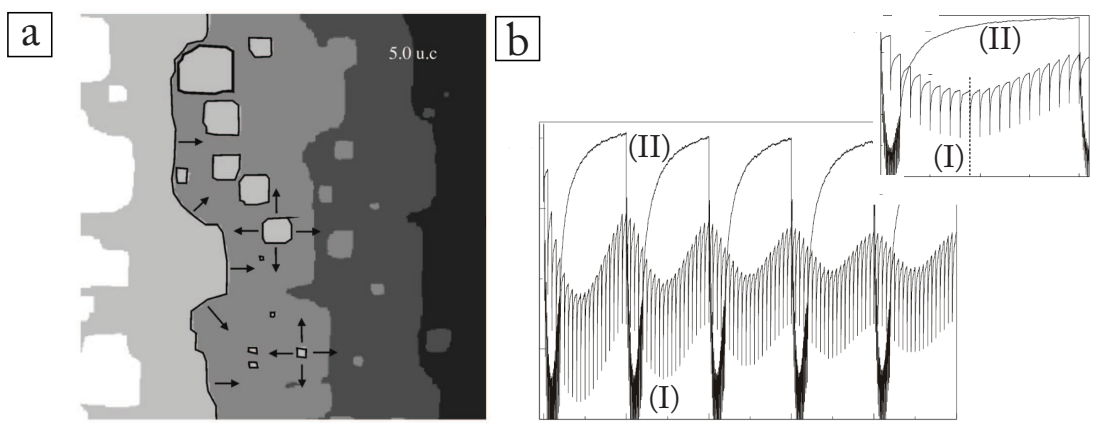

Fig. 2.6 KMC simulation of perovskite oxide thin film growth, a) KMC simulated surface topography image after five unit cells of deposited $\mathrm{SrRuO}_{3}$ on $\mathrm{SrTiO}_{3}(001)$, b) KMC simulated RHEED specular intensity of (I) $\mathrm{SrTiO}_{3}$ homoepitaxial PLD growth and of (II) $\mathrm{SrTiO}_{3}$ homoepitaxial pulsed laser interval deposition (PLID) growth. Inset shows a zoom-in on the RHEED specular intensity during both PLD and PLID growth of a single monolayer.

Calculating the hopping rate $k_{\text {hopping }}$ for adatoms is performed according to:

$$
k_{\text {hopping }}=k_{0} \cdot e^{\frac{E_{\mathrm{D}}+n \cdot E_{\mathrm{N}}}{k_{\mathrm{B}} T}}
$$

where, $k_{0}$ represents the attempt frequency for atomic processes, $E_{\mathrm{D}}$ is the energy barrier on the surface, $E_{\mathrm{N}}$ is the nearest neighbor energy and $n$ the number of neighbors $(0,1,2,3,4)$. The activation barrier energy for hopping has the form of $E_{\mathrm{D}}+n \cdot E_{\mathrm{N}}$ and scales with the number of neighbor (atomistic) interactions $n$. The RHEED signal $I_{\text {RHEED }}$ scales with the step density of a surface according to Eq. 2.5. The step density model for an $l \times l$ matrix in KMC simulations is calculated according to [146]:

$$
S=\frac{1}{l^{2}} \sum_{i, j}\left|h_{i, j}-h_{i, j+1}\right| \cos (\Phi)+\left|h_{i, j}-h_{i+1, j}\right| \sin (\Phi)
$$

where $S$ is the step density, $h_{i, j}$ the step heights on the surface and $\Phi$ the azimuthal angle of the electron beam with respect to the principal directions of the matrix.

During several decades, faster Monte Carlo algorithms have been written in order to minimize the simulation time [147]. Furthermore, algorithms have been developed to simulate $3 \mathrm{D}$ growth of i.e. $\mathrm{SrRuO}_{3}$ nanowires, while usually only inplane diffusion is considered [148]. The main algorithm difference between KMC 
simulations of PLD and MBE is the way new deposited material is added on the simulated surface. In PLD, the material on the surface is added in a zero duration "pulsed" manner (dependent on the deposition frequency), while during MBE a continuous flow of new material is added on the surface.

Many studies have been reported to explain oxide thin film growth using KMC simulations [18, 58, 148, 149, 150, 151, 152, 153, 154]. The RHEED intensity is simulated to elucidate the experimental obtained RHEED specular intensity during PVD of oxides. These studies have in common that the KMC surface topography images reveal information about growing islands and other microscopic events, which is impossible with RHEED. Therefore, it will be beneficial to use real-space diagnostic tools during PVD thin film growth [25].

\subsection{AFM monitoring of growth kinetics during PLD}

This section starts with an overview of real-space diagnostic tools available for growth monitoring and motivates why AFM has been selected. Thereafter, the basics of AFM, the use of AFM during PLD and high-speed AFM under oxide PLD conditions are discussed.

\subsubsection{Real-space diagnostics of oxide PLD growth}

In PLD, the growth front evolution depends on growth kinetics on the surface and on its turn by the nucleation density and island radius evolution over time. For example, tracking of the island radius over time, is a measure for the lateral growth speed of islands and is therefore a measure for the diffusion length of adatoms. On the other hand, monitoring the nucleation density gives an estimate of the supersaturation in PLD and its dependence on i.e sample temperature, deposition pressure and laser fluence etc. Real-space diagnostic tools have in common that the monitoring performance is independent on the material crystalline state. In addition, topographic features can be imaged down to atomic resolution.

Fig. 2.7 gives a schematic view of the PLD process. Just after the laser pulse, typically $25 \mathrm{~ns}$, the deposition pulse with a duration of several hundreds of $\mu \mathrm{s}$ takes place. In contrast to other deposition methods, growth can be studied in between these deposition pulses without being disturbed by new deposited material. During PLD, the high supersaturation induces a high nucleation density up to the critical nucleation density. In between the PLD deposition pulses, adatoms 


\begin{tabular}{c|ccccc}
\hline & SEM & TEM & LEEM & STM & AFM \\
\hline Oxide PLD conditions & - & - & - & - & + \\
Direct measurement in 3D & - & - & - & + & + \\
Non-destructive & - & - & - & + & + \\
(Sub)nanometer resolution & + & + & + & + & + \\
High temporal resolution & + & + & + & + & - \\
Electrically insulating film/substrate & + & + & + & - & + \\
\hline
\end{tabular}

Tab. 2.3 Suitability overview of real-space diagnostic tools to monitor oxide growth kinetic processes during PLD.

diffuse on an island to an island edge (lateral island growth) or form new nuclei centers on top of or in between islands (vertical island growth). Understanding the relation between the critical nucleation density, island radius with the growth front evolution would increase the understanding of oxide PLD thin film growth and thus tunability/control on (nano) functional materials.

A suitable microscopy technique to monitor the nucleation density and island
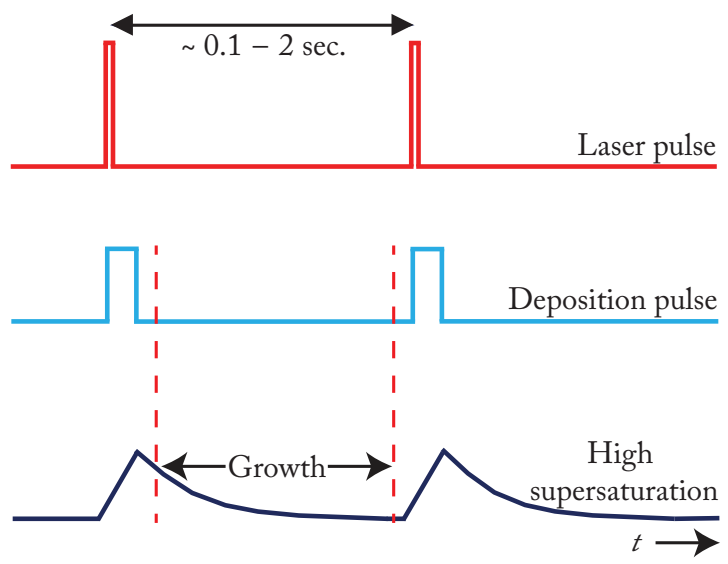

Fig. 2.7 Schematic view of PLD assuming that deposition and growth are separated in time $t$. The laser pulse, with a pulse width of typically $25 \mathrm{~ns}$, induces a plasma of ablated species existing for several hundreds of $\mu \mathrm{s}$. The supersaturated material is deposited on the substrate surface, whereafter growth takes place in between the deposition pulses. Monitoring the decay of the nucleation density and increase of island radius enables the measurement of growth kinetic parameters. 


\begin{tabular}{c|cc}
\hline Monitoring technique & RHEED & AFM \\
\hline Area & $>250 \mu \mathrm{m}$ & few $\mu \mathrm{m}$ \\
Parameter & Step density & Nucleation density \& radius \\
Ordering & Only crystalline & (Poly) crystalline \& amorphous \\
Space & Reciprocal & Real \\
Interpretation & "Indirect" & "Direct" \\
\hline
\end{tabular}

Tab. 2.4 Overview of (relative) characteristics of RHEED and AFM as diagnostic tools for growth monitoring during PLD.

radius during oxide PLD has to meet all the listed conditions: (1) operating at typical oxide PLD pressures (ranging from $10^{-6}-10^{-1}$ mbar $\mathrm{O}_{2}$ ) and temperatures (ranging from RT up to $850{ }^{\circ} \mathrm{C}$ ) (2) non-destructive for the sample surface (3) (sub)nanometer spatial resolution (4) high temporal resolution and (5) operating on electrically insulating films/substrates. Tab. 2.4 gives an overview of real-space diagnostics tools whether they meet the listed conditions. Real-space diagnostics tools, such as SEM, transmission electron microscopy (TEM), low-energy electron microscopy (LEEM) and scanning tunneling microscopy (STM) have been utilized to monitor a surface morphology, but so far none of them have been used under metal-oxide PLD conditions in contrast to atomic force microscopy (AFM) [33, 34]. The main differences between AFM and RHEED are that 1) the probing area of AFM is much smaller compared to RHEED and individual nanometer sized islands can be tracked, 2) The RHEED intensity provides an indirect measurement of the normalized step density $S / S_{\max }$, while AFM enables a direct measurement of the step density $S$, nucleation density $N_{\mathrm{s}}$ and radius $r_{\text {island }}$ evolution, 3) AFM operates with a performance independent of the crystalline state and is even applicable to amorphous materials, 4) real-space data gives a direct measure of length scales and 5) RHEED data is harder to interpreted due to dynamic scattering (complicating interpretation of RHEED patterns). AFM meets all listed conditions with exception of the temporal resolution (based on conventional AFM's). The typical time for an AFM to acquire an image is in the order of minutes, where characteristic times of oxide growth kinetic processes during PLD are in (sub)seconds [17, 18]. Recently, a lot of progress has been made to increase the temporal resolution of AFM, which subsequently will be discussed [39, 62, 63]. 


\subsubsection{AFM in general}

The atomic force microscope (AFM) belongs to the class of scanning probe microscopy (SPM) techniques. After the introduction in 1986, the invention of the AFM by Binnig et al. resulted in a new powerful microscopy tool for researchers to study materials independent of the band gap [30] as a follow up instrument on the earlier presented scanning tunneling microscope (STM) [31]. Nowadays, AFM has been utilized in various fields to study many processes and mechanisms at (sub)nanometer spatial resolution [39, 155, 156]. AFM enables surface mapping at high spatial resolution of properties, such as the surface topography, in liquid, air and even under vacuum conditions. In addition to imaging, AFM has been used to measure mechanical properties using force spectroscopy and i.e. electrical and magnetic surface properties have been measured.

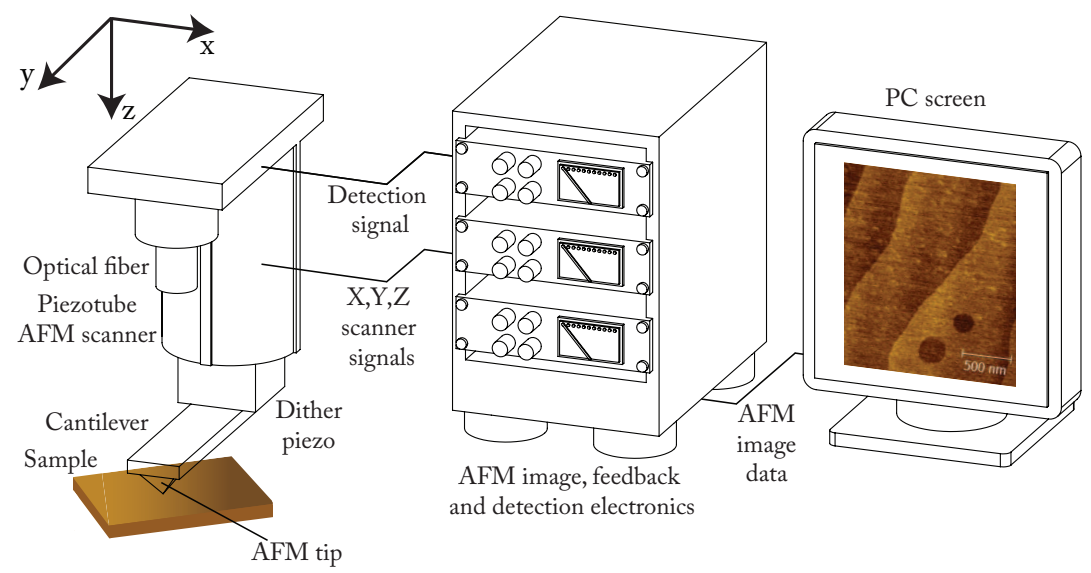

Fig. 2.8 The concept of AFM.

The basic AFM instrumentation is presented in Fig. 2.8. A sharp tip mounted on an elastically suspended probe, which is called the cantilever, is brought in close vicinity of the sample surface such that there acts an interaction force between tip and sample $F_{\text {ts }}$. The force on the tip results in a vertical probe displacement of the cantilever beam. The sharp tip and high force sensitivity of the beam allow the measurement of small forces enabling sub-nanometer resolution. Deflection of the cantilever beam can be measured by various detection methods, such as optical beam deflection (OBD) and interferometry. In Fig. 2.8, the interferometry detection is illustrated. An optical fiber is aligned above the cantilever in order to detect the cantilever motion properties. Light is emitted from a laser through the fiber onto the cantilever top surface. From the top surface the reflected wave from the cantilever is fed into the optical fiber together with the reflected wave from 
the fiber glass-air interface. Both reflected waves are detected by a photodetector in the interferometer. A sinusoidal signal from the photodetector is measured by a deflection (contact mode), amplitude $A_{\text {osc }}$ (tapping modulation, TM-AFM) or frequency shift $\Delta f$ (frequency modulation, FM-AFM) detector to measure one of these modulating cantilever properties. This signal is fed into the height feedback to keep the distance $d$ (and therefore $F_{\text {ts }}$ ) between AFM tip and sample constant. The minimum detectable force gradient $\partial F_{t s m i n}$ in both TM-AFM and FM-AFM is given by the subsequent equation:

$$
\partial F_{t s m i n}=\sqrt{\frac{2 k_{c} k_{B} T_{c} B_{c}}{f_{0} Q_{c}<A_{o s c}^{2}>}}
$$

where $k_{\mathrm{c}}$ is the cantilever spring constant, $k_{\mathrm{B}} T_{\mathrm{c}}$ the thermal energy, $B_{\mathrm{c}}$ the measurement bandwidth, $f_{0}$ the cantilever resonance frequency, $Q_{\mathrm{c}}$ the cantilever quality factor and $\left\langle A_{\mathrm{osc}}{ }^{2}\right\rangle$ the mean square cantilever amplitude. From Eq. 2.10, it can be seen that an increase in $Q_{\mathrm{c}}$ results in an increase in force gradient sensitivity.

In AFM, the average tip-sample interaction force $<F_{\text {ts }}>$ increases usually when the distance $d$ between AFM tip and sample surface becomes smaller [157]. San Paulo and Garcia derived an equation showing a relation between $\left\langle F_{\text {ts }}>\right.$ and the cantilever oscillation amplitude $A_{\mathrm{osc}}$ by taking into account that attractive and repulsive forces are only significant near the lower turning point of the oscillation in TM-AFM [158]:

$$
A_{\mathrm{osc}} \approx A_{0} \sqrt{\left(1-4\left(\frac{<F_{\mathrm{ts}}>}{F_{0}}\right)^{2}\right.}
$$

where, $A_{0}$ is the driving amplitude and $F_{0}$ is the amplitude of driving force. Typically, a small $A_{\mathrm{osc}}=0.1-10 \mathrm{~nm}$ is used during FM-AFM imaging. An approximation of the relation between $\Delta f$ and the $F_{\text {ts }}$ is given for small $A_{\text {osc }}$ according to [157]:

$$
\Delta f=-\frac{f_{0}}{2 k_{c}} \frac{\partial F_{t s}}{\partial d}
$$

where $f_{0}$ is the cantilever resonance frequency, $k_{c}$ is the cantilever spring constant. Qualitative force-distance curves can be made by measuring $\Delta f$ as function of distance $d$. Both Eqs. 2.11 and 2.12 show that the cantilever oscillation properties $A_{\mathrm{osc}}$ and $\Delta f$ are related to $<F_{\mathrm{ts}}>$ or $\partial F_{\mathrm{ts}} / \partial d$, respectively.

The typical modus operandi of an AFM is raster-scanning the AFM tip on the sample surface while monitoring $F_{\text {ts }}$ (either in TM-AFM or FM-AFM) and keep 
$F_{\text {ts }}$ constant using the PID controller. Scanning of the AFM tip is controlled by the piezotube ${ }^{5}$ (separated piezoelements in the X,Y,Z direction). In AFM, the raster scanning is generated by applying a sawtooth signal in the $\mathrm{X}$-direction and a voltage ramp in the $\mathrm{Y}$-direction.

Two AFM scanning principles can be used, namely constant force and constant height mode. During constant force (closed loop) mode, the AFM feedback regulates the voltage over the Z-piezo to adjust distance $d$ to maintain $F_{\text {ts. }}$. The position of the Z-piezo is acquired by the electronics as a function of the $\mathrm{X}$ and $\mathrm{Y}$ scanner position and translated to a topographical image represented on the $\mathrm{PC}$ screen. In constant height (open loop) AFM mode, the position of the Z-piezo is kept constant during raster scanning. Then one of the modulating properties is a measure for the sample surface topography. Constant force mode gives a better Z-resolution, while constant height enables faster AFM acquisition rates [37]. High resolution AFM is only possible if the AFM setup has a sufficiently low vibrational level. In general, this is achieved by an as rigid as possible AFM construction and the use of active and passive damping systems.

\subsubsection{In situ AFM under oxide PLD conditions}

Only a single work has been reported of an AFM operating at typical oxide PLD conditions [33]. This work is mainly focused on the applicability of AFM on $\mathrm{ABO}_{3}$ crystalline surfaces under oxide PLD conditions and imaging during PLD. To prevent an AFM scanner breakdown of the piezotube scanner, Broekmaat et al. placed a macor tube as thermal insulator on top of the piezotube scanner to reduce heating of the piezoelements. Furthermore, a high temperature sample stage has been designed to prevent warming of the piezotube scanner by minimizing the required heater input power to reach a certain $\mathrm{ABO}_{3}$ sample temperature [34]. They reported that $\mathrm{SrTiO}_{3}(001)$ can be imaged up to at least $750{ }^{\circ} \mathrm{C}$ and at $10^{-5}$ $10^{-1}$ mbar $\mathrm{O}_{2}$ by a proper scanner and heater design [34]. However, they observed an AFM instability (under PLD growth conditions) between AFM tip and sample surface of i.e. $\mathrm{SrRuO}_{3}$, a widely used electrode material in $\mathrm{ABO}_{3}$ heterostructures. Switching from TM-AFM to contact mode (CM) AFM revealed that blobs have been formed on the surface after so-called "neck formation", where earlier blobs were observed by Kuipers et al. during STM at elevated temperatures [159].

An AFM scanning instability is reported for oxides at elevated temperatures ${ }^{6}$,

\footnotetext{
${ }^{5}$ Piezoelectric materials can contract or expand by applying a voltage over the material.

${ }^{6}$ In this work, neck formation has been observed on mixed termination $\mathrm{SrTiO}_{3}$ at $T=600{ }^{\circ} \mathrm{C}$ and $\mathrm{PO}_{2}=10^{-1} \mathrm{mbar}_{2}$. It is explained by the fact that a neck is formed with $\mathrm{SrO}$ as $\mathrm{SrRuO}_{3}$ has a $\mathrm{SrO}$ terminating layer, see Ch. 3 .
} 

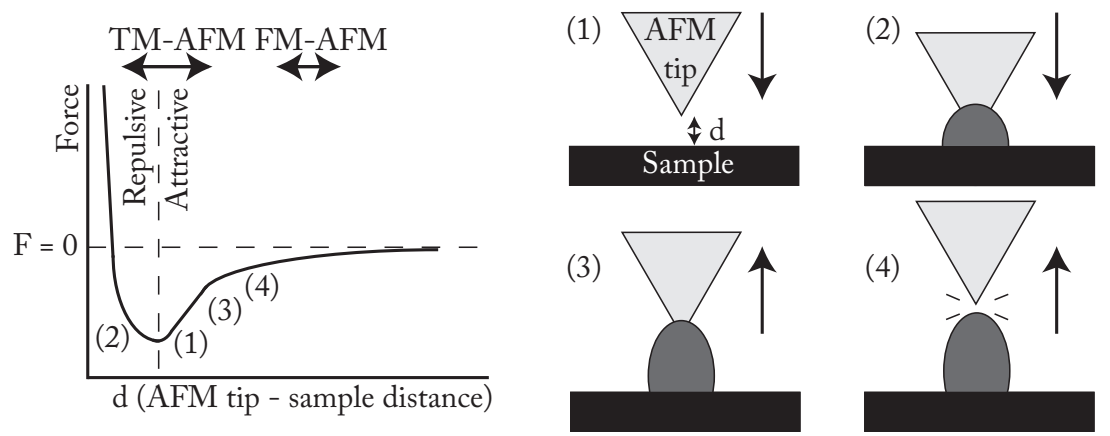

Fig. 2.9 Schematic illustration of operation distance $d$ between AFM tip and sample surface in TM-AFM and FM-AFM. The probability of neck formation shows a dependence on the distance $d$ between AFM tip and sample [33, 159]. (1) Approach of the tip, (2) just after the jump into contact, (3) growth of a neck and (4) breaking of the neck.

such as $\mathrm{SrRuO}_{3}\left(\geq 500-600{ }^{\circ} \mathrm{C}\right)$, undoped ITO $\left(\geq 300-400{ }^{\circ} \mathrm{C}\right)$ using a Si AFM tip [33]. Changing the AFM tip material from a bare Si tip to a Pt coated Si tip resulted in an AFM instability temperature above $400{ }^{\circ} \mathrm{C}$ on $\mathrm{SrTiO}_{3}(001)$, while with the bare Si tip it could be imaged up to $750{ }^{\circ} \mathrm{C}$ at $10^{-2}$ mbar $\mathrm{O}_{2}$ [33]. For metals, an STM instability is observed with a $\mathrm{W}$ tip on a $\mathrm{Pb}(110)$ surface at a temperature of $45^{\circ} \mathrm{C}$ [159]. Thereby, Broekmaat et al. used a conventional AFM with a low AFM feedback bandwidth and it is claimed that slow AFM tip velocity increases the probability of neck formation [33].

Several recommendations are given in order to reduce/prevent neck formation on $\mathrm{ABO}_{3}$ surfaces under oxide PLD conditions. The first recommendation is the use of FM-AFM (attractive force regime) instead of TM-AFM (intermittent force regime). The attractive interaction strength between AFM tip and sample is smaller compared to the intermittent regime as the distance between tip and sample is larger, see Fig. 2.9. Therefore, it is expected that the probability to form a neck will decrease. The operation of FM-AFM under oxide PLD conditions compared to TM-AFM is more complicated. During TM-AFM, $A_{\mathrm{osc}}$ is by far the most critical parameter to tune, but in FM-AFM more parameters have to be optimized, such as $A_{\text {osc }}, \Delta f$ and $\tau_{\text {demod }}[155]$.

Second recommendation is the selection of a suitable tip material/coating. Changing the tip coating and/or film/substrate material affects the conditions at which an AFM operation stability is achieved. Therefore, more understanding has to be gathered about the phenomena (chemical reactions, surface energies), which play a role during AFM imaging under metal-oxide PLD conditions. The third recommendation is the increase of the vertical and lateral tip velocity. Increasing the tip 
velocity will reduce the probability to form a neck. It is not only a requirement to prevent neck formation, but also for monitoring of oxide growth kinetic processes occurring at characteristic relaxation times $\tau$ [17]. Higher AFM tip velocities can be achieved by the development of high-speed AFM instrumentation.

\subsubsection{High-speed AFM under PLD conditions}

High-speed AFM (HS-AFM) instrumentation is developed in order to visualize nano-scale dynamics (timescale $\approx$ hundreds of $\mathrm{ms}$ ) $[35,39,62,64,63,160]$. A lot of progress is made to speed up the AFM acquisition rate. Both the development of HS-AFM instrumentation and the HS-AFM operation under metal-oxide PLD conditions are discussed.

\section{Instrumentation for HS-AFM}

The AFM instrumentation consists mainly of four devices namely the cantilever, AFM detection system, an AFM scanner (head) and AFM electronics. All these components are related to the time delays involved in the feedback loop bandwidth described in Eq. 2.18 and Fig. 2.11. To extend the maximum AFM acquisition rate $R_{\max }$, many groups focused on increase of the cantilever resonance frequency $f_{0}[36,167,168]$, the scanner resonance frequency $f_{\mathrm{s}}[40,61]$ and increasing the bandwidth of the detection system $[167,169,170]$ and AFM electronics [35, 171]. Several research groups demonstrated the possibility of increasing the AFM acquisition rate $R_{\max }$ towards frames/s, see Tab. 2.5.

One way to increase $R_{\max }$ is reducing the cantilever size and therefore increasing $f_{0}$. The resonance frequency $f_{0}$ of a rectangular cantilever depends on thickness $d_{\mathrm{c}}$, width $w_{\mathrm{c}}$ and length $L_{\mathrm{c}}$ according to [39]:

$$
f_{0} \sim \frac{d_{\mathrm{c}}}{L_{\mathrm{c}}^{2}}
$$

and,

$$
k_{\mathrm{c}} \sim w_{c}\left(\frac{d_{c}}{L_{c}}\right)^{3}
$$

Typically, $d_{\mathrm{c}}, w_{\mathrm{c}}$ and $L_{\mathrm{c}}$ of cantilevers are reduced to increase $f_{0}$ (keeping a similar $k_{\mathrm{c}}$ ) and therefore the AFM feedback bandwidth $f_{\mathrm{B}}$. For example, low stress SiN composite cantilevers with a length of $10 \mu \mathrm{m}$ and $f_{0}$ up to $2 \mathrm{MHz}$ are commercial 


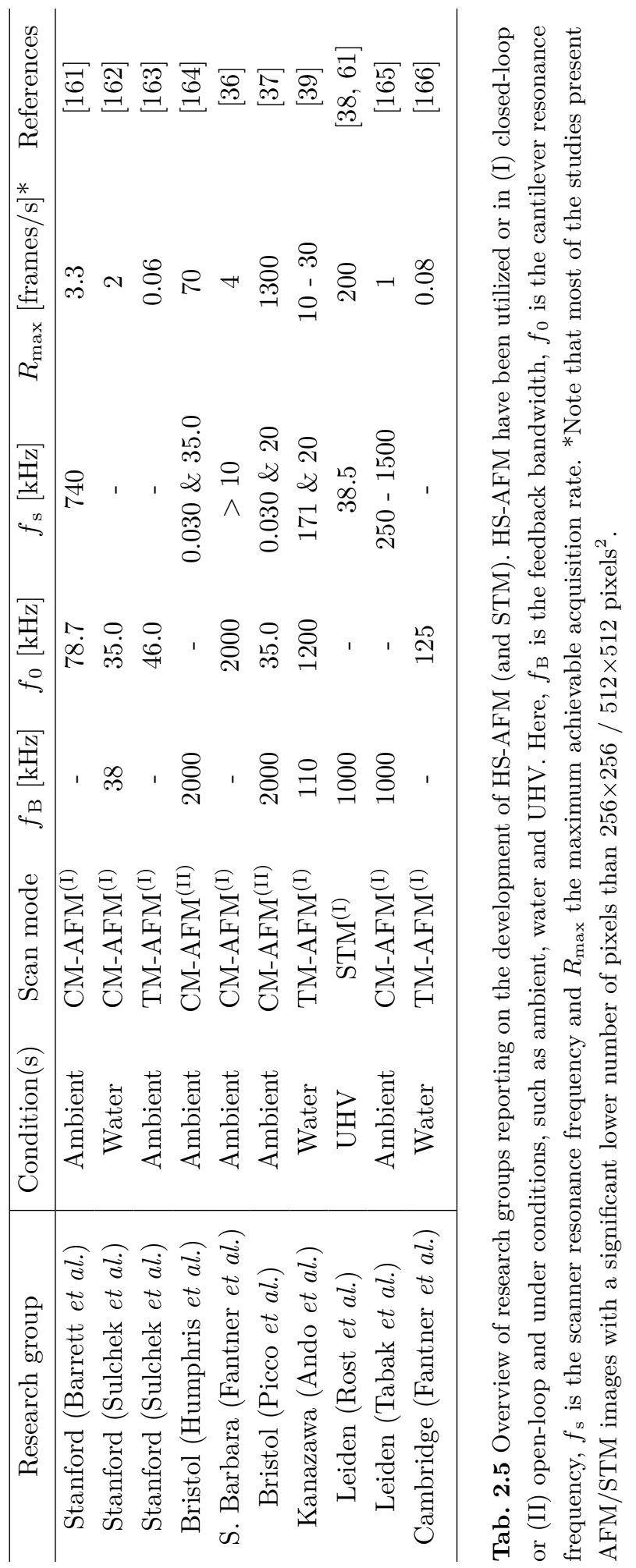



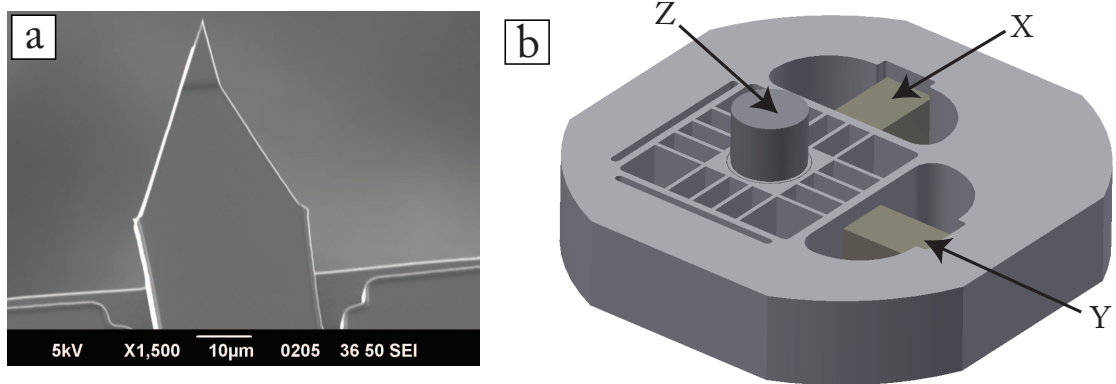

Fig. 2.10 Examples of mechanical AFM instrumentation to increase the maximum achievable acquisition rate $R_{\max }$, a) SEM image of an AC55TN high resonance frequency (small) cantilever of Olympus having a $f_{0}=1.6 \mathrm{MHz}($ scale $=10 \mu \mathrm{m})$, b) drawing of a flexure scanner design. AFM flexure scanners have been developed to extend $f_{\mathrm{s}}$.

available [36]. Si cantilevers containing a $f_{0}=2-5 \mathrm{MHz}$ were developed by several research groups $[62,172,173]$. Another way to increase the bandwidth (BW) of an AFM cantilever (BW $\left.\sim \frac{f_{0}}{Q_{c}}\right)$ is increasing the cantilever damping by selecting a different cantilever material and mimic a high $Q_{\mathrm{c}}$ factor [66]. Moreover, $R_{\max }$ has been increased by phase shifting the excitation signal using methods, such as $Q_{\mathrm{c}}$ control $[163,174,175,176]$. Jaafar et al. report that switching the AFM feedback on the drive-amplitude signal (DAM-AFM) (instead of cantilever oscillation amplitude $A_{\text {osc }}$ signal) reduces the cantilever response time in vacuum as $\tau_{\text {cDAM }} \approx \tau_{\text {cFM }}[177]$.

Besides achieving a higher $R_{\max }$, cantilevers with a higher $f_{0}$ (higher spring constant $k_{\mathrm{c}}$ ) have a lower total noise density $\sqrt{k_{\mathrm{B}} T / k_{\mathrm{c}}}[178]$. The total thermal noise is distributed over frequencies up to slightly above $f_{0}$ and therefore thermal noise is lower for higher $f_{0}$ cantilevers [167]. It has to be taken into account that cantilevers with $w_{\mathrm{c}} \leq 2 \mu \mathrm{m}$ reach the optical diffraction limit to align the optical beam on the cantilever using beam deflection detection method [39].

To detect oscillation properties of small $\mathrm{MHz}$ frequency cantilevers, the detector bandwidth should be above $f_{0}$ and the optical beam diameter should be smaller than the cantilever dimensions. Schäffer et al. were one of the first designers of an optical beam deflection (OBD) detector for small cantilevers [179]. In this design, single lenses are used for focusing the incident beam on the cantilever and reflective beam on the OBD detector. Later on, Ando et al. replaced the single lens by an objective lens (working distance $=8 \mathrm{~mm}$ ) and used a focused spot of 3 - $4 \mu \mathrm{m}$ in diameter [169]. A four-segment Si PIN photodiode (3 pF, $40 \mathrm{MHz})$ and a fast amplifier/signal conditioner $(\approx 20 \mathrm{MHz})$ were integrated in a photosensor to detect the deflection of $\mathrm{MHz}$ frequency cantilevers [39]. Hoogenboom et al. 
developed a Fabry-Perot interferometer for micrometer-sized cantilevers [170]. The interferometer has a spot size of only $3 \mu \mathrm{m}$ in diameter and the photodetector had a noise floor of $1 \mathrm{fm} / \sqrt{\mathrm{Hz}}$ at $1 \mathrm{MHz}$. The signal coming from the photodetector is fed into a RMS-to-DC converter, which detects the cantilever oscillation amplitude $A_{\text {osc }}$ (DC output voltage is a measure for $A_{\text {osc }}$ ) or a phase-locked-loop (PLL) outputs a DC signal as measure for the frequency shift $\Delta f$.

Detecting the cantilever oscillation amplitude costs at least half an oscillation period according to $\tau_{\mathrm{m}}=1 / 2 f_{0}$ [39]. Most amplitude detectors (RMS-to-DC converters) require several oscillation cycles to output an accurate RMS value. Groups introduced a $10 \mathrm{MHz}$ peak-hold method (also called peak-detector) and reduced the required cycles to only half a cycle [169] and developed a Fourier method for fast amplitude/frequency shift detection [180]. Recently, Karvinen et al. have become inspired by the RF and microwave circuit design and they introduced a phase shift of $90^{\circ}$ to the input signal resulting in canceling of the $2 \omega_{0}$ component and no filtering was required (and increase of measurement bandwidth) [181]. Nowadays, PLL demodulator bandwidths are achieved of $0.2-5 \mathrm{MHz}$ by the company Zurich Instruments and the research group of Fantner [65, 182]. Next to that, other high bandwidth electronics is needed for acquiring and applying higher frequency AFM scanner signals.

Several groups developed high bandwidth AFM electronics [35, 164, 169, 171, 183]. Rost et al. developed a video-rate ( $\geq 25$ frames/s) SPM controller enabling (realtime) monitoring of thin film growth [25, 184] and catalysis [185, 186]. The videorate controller existed of an $\mathrm{MHz}$ bandwidth analog feedback electronics and a piezodriver to reach a tip speed of $0.3 \mathrm{~m} / \mathrm{s}$, while obtaining atomic resolution [35]. In the field of biomolecule studies, Ando and coworkers are leading inventors of HS-AFM [39]. After years of pushing the AFM speed limits, Ando et al. published high-speed tapping mode AFM (HS-TM-AFM) observations of a walking myosin $\mathrm{V}$ biomolecule occurring in hundreds of ms [187]. They designed a dynamic high bandwidth $(70 \mathrm{kHz})$ PID controller to optimize the PID settings continuously during AFM imaging [188]. A dynamic PID controller reduced the feedback saturation and there is a limited bandwidth dependence on cantilever amplitude. Furthermore, it reduced the parachuting time $\tau_{\mathrm{p}}$ as the gain should be larger at downhill regions compared to uphill regions [39]. Picco et al. achieved one of the highest reported $\mathrm{AFM}$ acquisition rates $(\approx 1000$ frames/s) with their developed instrumentation by using contact mode AFM in open loop [37]. In that case, the AFM scan speed is mainly limited by the mechanical microscope/scanner.

Piezotube scanners have low resonance frequencies around several hundreds of $\mathrm{Hz}$ due to the elongated tube shape [38]. Other scanner designs have been proposed 
in literature in order to extend $f_{\mathrm{s}}$. Conical piezotubes have a triangular aspect of shape and therefore a higher stiffness design and higher $f_{\mathrm{s}}$ compared to conventional piezotubes $[38,61]$. A first resonance $f_{\mathrm{s}}$ has been found at $45 \mathrm{kHz}$ in Ref. [61]. Flexure hinges (see Fig. 2.10 b)) are designed with the desire to increase stiffness and decoupling of the X, Y and Z [39, 40, 62, 189]. Dependent on the required scan range, the first resonance $f_{\mathrm{s}}$ is typically found at tens of $\mathrm{kHz}$. A MEMS design with $f_{\mathrm{s}}=1.5 \mathrm{MHz}$ is presented as a Z-scanner, which has a mass of only $10^{-11} \mathrm{~kg}[165]$.

\section{Feedback operation of HS-AFM}

HS-AFM is defined as AFM imaging with a frame rate beyond 10 frames/s having $256 \times 256$ pixels $^{2}[38,171]$. Conventional AFM's were slow due to the low feedback bandwidth and therefore it takes a few minutes to record a single AFM image [38]. The AFM feedback bandwidth depends on the bandwidths of instrumentation, such as the AFM scanner, AFM electronics, AFM cantilevers and (optical) detection system. In order to achieve an increase in temporal AFM resolution, the bandwidths of these components have to be significantly increased [36]. All timeconstants of the feedback loop have to be as low as possible, see Fig. 2.11. The AFM feedback can be considered as a chain, which is just as strong as the weakest link [190].

As an example, from the AFM image settings and sample surface topography, the required AFM feedback bandwidth $f_{B}$ can be calculated. Suppose that an AFM image is recorded in time $t_{\mathrm{AFM}}$, over a $W \times W$ scan range with $N$ scan lines. The scan velocity $V_{\mathrm{s}}$ in the x-direction is then given by $V_{\mathrm{s}}=2 \times W \times N / t_{\mathrm{AFM}}$ [39]. In the case a sinusoidal shape sample surface (periodicity $=\lambda_{\text {surf }}$ ) is assumed, the feedback operation frequency $f=V_{\mathrm{s}} / \lambda_{\text {surf }}$ in order to maintain the tip-sample distance. Therefore, the feedback bandwidth $f_{\mathrm{B}}$ has to be equal to or larger than $f$ according to:

$$
f_{\mathrm{B}} \geq \frac{2 W N}{\lambda_{\mathrm{surf}} t_{\mathrm{AFM}}}
$$

The expression in Eq. 2.15 describes the relation between the AFM acquisition time $t_{\mathrm{AFM}}$ and feedback bandwidth $f_{\mathrm{B}}$. From Eq. 2.15, it becomes clear that the required $f_{\mathrm{B}}$ is related to spatial frequency in the surface morphology. From this the maximum achievable AFM imaging rate $R_{\max }\left(1 / t_{\mathrm{AFM}}\right)$ can be calculated: 


$$
R_{\max }=\frac{\lambda_{\text {surf }} f_{\mathrm{B}}}{2 W N}
$$

A $f_{\mathrm{B}}=110 \mathrm{kHz}$ is reported by Ando et al. and is approximately three orders of magnitude higher than conventional AFM's [63]. Assuming a $\lambda_{\text {surf }}=100 \mathrm{~nm}$, $W=1 \mu \mathrm{m}$ and $N=512$ lines results in maximum achievable AFM acquisition rate $R_{\max }=10$ frames $/ \mathrm{s}$.

\section{TM-HS-AFM under metal-oxide PLD conditions}

Dynamic mode AFM is preferred instead of contact mode AFM under metaloxide PLD conditions as contact mode is more sample destructive due to large shear forces [33]. TM-AFM is easier in use compared to FM-AFM as less parameters need to be adjusted. TM-AFM was proven as AFM scan mode to image $\mathrm{SrTiO}_{3}(001)$ at $T=750{ }^{\circ} \mathrm{C}$ and $\mathrm{PO}_{2}=10^{-2}$ mbar $\mathrm{O}_{2}$ [34]. However, TM-HS-AFM under metal-oxide PLD conditions requires an increase in feedback bandwidth $f_{\mathrm{B}}$. Ando et al. presented an approximation of $f_{\mathrm{B}}$ dependent on various factors under ambient conditions [39]. This approximation can be applied for TM-HS-AFM under metal-oxide PLD conditions as well.

First of all, there is always a phase delay $\phi(\omega)$ in open-loop. A relation of $\varphi(\omega)=2 \phi(\omega)(\varphi(\omega)$ is phase delay closed-loop) is given by satisfactory of the feedback performance and the feedback gain is maintained at $\approx 1$ (a detailed description can be found in Ref. [39]). The open-loop phase delay is described by $\phi(\omega)=2 \pi f \Delta \tau$, where $\Delta \tau$ is the total time delay in open-loop. In Fig. 2.11, the main time delays in TM-AFM are indicated, where $\tau_{\text {cTM }}$ is the cantilever response time in TM-AFM, $\tau_{\mathrm{m}}$ is the reading time of the cantilever oscillation amplitude (reading of the cantilever oscillation amplitude needs at least a time of $\tau_{\mathrm{m}}=\frac{1}{2 f_{0}}$ ), $\tau_{\text {I }}$ is the integral time of error signals in the feedback controller, $\tau_{\mathrm{z}}$ is the $\mathrm{z}$-scanner response time and $\tau_{\mathrm{p}}$ is the parachuting time. Parachuting is here described as that the cantilever tip completely detaches from the sample surface at a steeply sample inclined region and then time elapse until it lands on the surface again [39].

The cantilever and z-scanner behave as second-order resonant systems [62]. As a result, the cantilever response time $\tau_{\text {cTM }}$ (in TM-AFM) is expressed as:

$$
\tau_{\mathrm{cTM}}=\frac{Q_{\mathrm{c}}}{\pi f_{0}}
$$

where $Q_{\mathrm{c}}$ is the cantilever $Q$ factor. The z-scanner response time is given by $\tau_{\mathrm{z}}=\frac{Q_{\mathrm{S}}}{\pi f_{\mathrm{S}}}$, where $Q_{\mathrm{s}}$ is the scanner $Q$ factor and $f_{\mathrm{s}}$ is the scanner resonance 


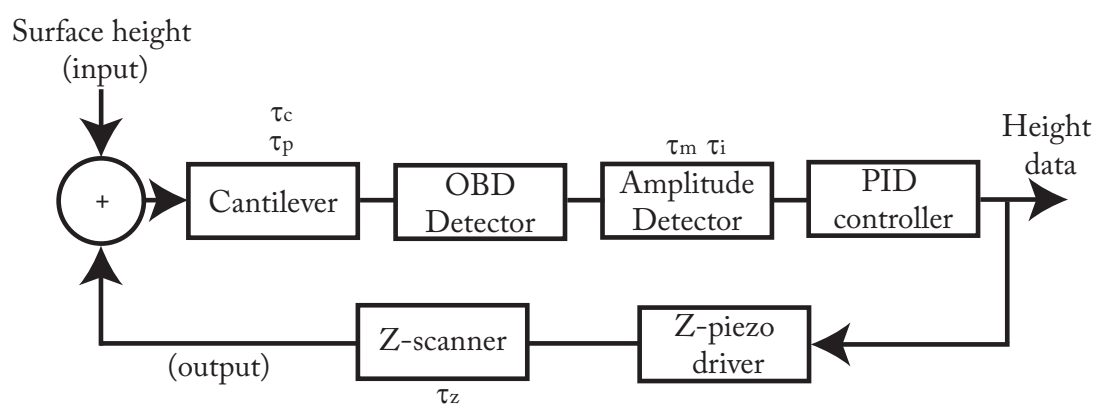

Fig. 2.11 Feedback loop of TM-AFM and the involved time delays. The height variation of the surface is taken as input for the feedback loop and the latter is controlling the $\mathrm{z}$ scanner as output. The most important time delays in the feedback loop are the cantilever response time $\tau_{\mathrm{cTM}}$, parachuting time of the cantilever $\tau_{\mathrm{p}}$, time required to measure the cantilever oscillation amplitude detection time $\tau_{\mathrm{m}}$, the integral time of error signal of the PID controller $\tau_{\mathrm{I}}$ and the z-scanner response $\tau_{\mathrm{z}}$.

frequency. An approximation of feedback bandwidth $f_{\mathrm{B}}$ is given in Eq. 2.18, the feedback frequency having a time delay of $\frac{\pi}{4}$ :

$$
f_{\mathrm{B}}=\alpha \frac{f_{0}}{8}\left(1+\frac{2 Q_{\mathrm{c}}}{\pi}+\frac{2 Q_{\mathrm{s}} f_{0}}{\pi f_{\mathrm{s}}}+2 f_{0}\left(\tau_{\mathrm{p}}+\tau_{\mathrm{I}}+\delta\right)\right)
$$

The cantilever resonance frequency $f_{0}$ is involved in the feedback delays $\tau_{\text {cTM }}$, $\tau_{\mathrm{cFM}}$ (cantilever response time in frequency-modulation AFM) and $\tau_{\mathrm{m}}$ (reading time of cantilever oscillation amplitude TM-AFM) and are inversely proportional to $f_{0}$. Guidelines for developing a TM-HS-AFM are presented [39]:

- Increase of cantilever resonance frequency $f_{0}$. This is the most important parameter to extend the feedback bandwidth $f_{\mathrm{B}}$.

- $Q$ factors of cantilever $Q_{\mathrm{c}}$ and z-scanner $Q_{\mathrm{s}}$ have to be lowered.

- Resonance frequency of z-scanner $f_{\mathrm{s}}$ should be high. Preferably above $f_{0}$.

- An additional phase compensator that can compensate for the phase delay.

- The free oscillation peak-to-peak amplitude of the cantilever should be a few times larger than the maximum height of topographic features.

- Avoid or shorten parachuting time $\tau_{\mathrm{p}}$.

- Electronic systems should have bandwidths preferably in the order of $\mathrm{MHz}$. 
Increasing the scanner and cantilever resonance frequencies $\left(f_{\mathrm{s}}\right.$ and $f_{0}$, respectively) has the highest priority [39]. Under metal-oxide PLD conditions, another priority is facing a high $\tau_{\text {cTM }}$ due to the high $Q_{\mathrm{c}}$. The cantilever $Q_{\mathrm{c}}$ can be $\geq 10^{4}$ under metal-oxide PLD conditions, while in ambient it is $\approx 10^{2}$ [178]. Assuming a $f_{0}=190 \mathrm{kHz}$ results in $\tau_{\mathrm{cTM}} \geq 17 \mathrm{~ms}$ and $\tau_{\mathrm{cTM}}=0.17 \mathrm{~ms}$ in vacuum and ambient, respectively.

\section{FM-HS-AFM under metal-oxide PLD conditions}

An alternative dynamic AFM scan mode is frequency-modulation (FM) AFM [178]. In FM-AFM, the cantilever response time $\tau_{\mathrm{cFM}}$ is similar under vacuum and ambient conditions. This AFM scan mode uses almost the same feedback scheme as presented in Fig. 2.11. The difference in feedback scheme is the replacement of the amplitude detector by a PLL to measure the frequency shift $\Delta f$. Therefore, the AFM feedback is performed at a constant $\Delta f$ setpoint instead of a constant A. FM-AFM is typically used in vacuum to circumvent the high cantilever response time $\tau_{\mathrm{cFM}}$. The cantilever response time for FM-AFM $\tau_{\mathrm{cFM}}$ in vacuum is independent of the cantilever $Q_{\mathrm{c}}$ :

$$
\tau_{\mathrm{cFM}}=\frac{1}{\pi f_{0}}
$$

which makes it more suited at UHV pressures. The cantilever response time in FM-AFM $\tau_{\text {cFM }}=1.7 \mu \mathrm{s}$, using a $f_{0}=190 \mathrm{kHz}$. FM-AFM is more difficult to operate as more parameters have to be optimized during AFM imaging, such as $A, \Delta f, \tau_{\mathrm{PLL}}$ (time-constant PLL) [155]. Furthermore, the PLL demodulator bandwidth was low until recently and therefore was limiting the maximum AFM acquisition rate $R_{\max }$ during FM-AFM. In addition, typically a small full-width half maximum (FWHM) of the amplitude vs. frequency curve (and therefore high $Q_{\mathrm{c}}$ ) is used to obtain high resolution AFM images. In order to image with HSFM-AFM under metal-oxide PLD conditions, the PLL demodulator bandwidth has to be larger than the required pixel rate. In general, ex situ AFM images contain $512 \times 512$ pixels $^{2}$ when recording perovskite oxide surface topographies. A PLL demodulator bandwidth of $263 \mathrm{kHz}$ is required when AFM images are to be acquired with one frame/s. 


\subsection{Conclusions}

PVD techniques, such as MBE and PLD have been successfully utilized in growing functional oxide materials. Especially, perovskite oxide materials are interesting due to the wide range in properties and their small variety in in-plane lattice parameter. MBE and PLD are popular PVD techniques as they allow growing oxide films with atomically flat surfaces and interfaces. In order to extract the growth behavior, in situ diagnostic tools, such as RHEED and SXRD have been adapted on both MBE and PLD. These diagnostic tools reveal the growth speed and growth front evolution only if the film material is nearly perfect crystalline.

AFM has the advantage that it can monitor the growth front evolution independent of the film crystalline state. Furthermore, topographic features with (sub)nanometer resolution can be imaged, while this is not feasible for reciprocal diagnostic tools. However, AFM on the same location as PVD results in hindering of deposition and most likely tip contamination. As deposition and growth in PLD are assumed to be separated in time, it allows to geometrically separate the AFM and PLD target in location. Therefore, growth can be monitored in between deposition pulses. Previously, it has been shown that perovskite oxide surfaces can be imaged using AFM under typical metal-oxide PLD conditions, but the maximum achievable acquisition rate is low. Recently, a lot of progress has been made to extend the maximum achievable AFM acquisition rate to several frames/s enabling the opportunity to monitor the oxide PLD growth kinetics with characteristic times in the order of (sub)seconds. 


\section{Part II}

Instrumentation \& Methods 



\title{
Chapter 3
}

\section{The development of an \\ atomic force microscope to study oxide growth during pulsed laser deposition}

\begin{abstract}
An atomic force microscope (AFM) has been developed to monitor the oxide growth front during pulsed laser deposition (PLD). The AFM scanner and PLD target are integrated in a single support frame such that microscopy can be utilized after subsequent deposition pulses. A sample transfer stage has been developed to transfer the sample back and forth between AFM and PLD. Microscopy can be performed from room temperature (RT) up to $700{ }^{\circ} \mathrm{C}$ and at pressures ranging from a base pressure of $10^{-6}$ mbar up to 1 mbar using gasses, such as $\mathrm{O}_{2}$ and Ar. The AFM performance is demonstrated by resolving $0.4 \mathrm{~nm}$ unit cell height $\mathrm{SrTiO}_{3}(001)$ surface steps and the surface topography of a $\mathrm{BiFeO}_{3}$ film under typical oxide PLD growth conditions.
\end{abstract}

W.A. Wessels, T.R.J. Bollmann, G. Koster and G. Rijnders, Real-time monitoring of thin film growth, NEVACBLAD 53, 6 (2015). 


\subsection{Introduction}

Atomic force microscopy (AFM) has been utilized in several research fields to study surfaces under different conditions, such as vacuum, air and liquids, since its invention in 1986 [30]. This popular research instrument enables probing of surfaces with (sub)nanometer resolution in real-space. Example of an AFM application is the imaging of a surface morphology after thin film deposition. Also in the field of oxide surfaces, films are imaged after deposition by applying ex situ AFM in air and/or in situ AFM at ultra high vacuum (UHV) [17, 18].

In pulsed laser deposition (PLD), deposition and growth are separated in time allowing monitoring of film growth without disturbing the deposition of new material (see Fig. 2.7 and Ch. 2) [33]. Nowadays, reflection high-energy electron diffraction (RHEED) is often utilized for real-time monitoring of oxide thin film growth [18]. However, the diffraction signal contains information especially about the growth mode, but information about e.g. ripening and defect formation is lacking. Studies reveal that real-space surface monitoring during other deposition methods results in increased thin film growth understanding, especially about these effects [25, 184].

Under oxide PLD conditions, AFM is not straightforward as oxide thin films are grown at elevated temperatures (typically $T=500-850{ }^{\circ} \mathrm{C}$ ) and at background pressures of $\left(10^{-6}-1\right.$ mbar) $\mathrm{O}_{2}[17,18,126,191]$. Some AFM considerations have to be made to image an oxide surface under oxide PLD conditions, namely thermal stabilization of the setup and it should operate at a wide range of oxygen background pressures. The stabilization time is the time required before a setup is in a working thermal equilibrium during sample heating [33]. This time depends on the stabilization time of 1) cantilever resonance frequency, 2) heating stage and (3) mechanical setup. Next to that, the breakdown voltage of piezoelectric elements, typically used for cantilever positioning by the AFM scanner, varies with operating (oxygen) background pressure and separation distance between its electrodes according to Paschen's law [192].

A first AFM design and demonstration under typical oxide PLD conditions is reported $[33,34]$. Their developed AFM based on a conventional (EBL2\#) piezotube scanner with a thermally insulating macor tube between piezotube and cantilever holder unit. The macor tube was placed between the piezotube and cantilever holder unit to prevent heating of the scanner piezo elements and therefore avoiding piezo depolarization. Interferometric detection was used to measure cantilever deflection as beam deflection is unpractical under oxide PLD conditions: (1) the AFM scanner has a more compact design and (2) no adjustment of mirrors 
needed. Contact mode (CM) and Tapping mode (TM) AFM were performed on $\mathrm{SrTiO}_{3}(001)$ and $\mathrm{DyScO}_{3}(110)$ substrates at controlled vacuum pressures $\left(10^{-5}\right.$ $10^{-1}$ mbar $\mathrm{O}_{2}$ ) and up to $T=750{ }^{\circ} \mathrm{C}$, which are typical PLD conditions to grow perovskite oxides, such as $\mathrm{SrRuO}_{3}, \mathrm{SrTiO}_{3}$ and $\mathrm{BiFeO}_{3}[18,126,191]$.

However, an attempt to image a $\mathrm{SrRuO}_{3}(001)$ thin film above $T=500{ }^{\circ} \mathrm{C}$ at $10^{-2}$ mbar $\mathrm{O}_{2}$ revealed that a neck was formed between a Si AFM tip and $\mathrm{SrRuO}_{3}$ thin film surface [159]. Neck formation causes an AFM operation instability and therefore it was not possible to image $\mathrm{SrRuO}_{3}(001)$ under oxide PLD conditions. When $\mathrm{Pt} / \mathrm{Ir}$-coated Si tips were used, it turned out that $\mathrm{SrTiO}_{3}(001)$ and $\mathrm{DyScO}_{3}(110)$ could only be imaged up to $T=500{ }^{\circ} \mathrm{C}$ using these AFM tips. This study concluded that neck formation can be circumvented by (1) selecting a suitable tipsample combination, (2) frequency-modulation (FM) AFM instead of TM-AFM or (3) increase of AFM tip velocities during raster scanning, see Ch. 2 [33, 39]. The latter is also a requirement in order to monitor oxide growth kinetic processes during PLD using AFM.

The acquisition rate of the reported AFM operating at PLD conditions is low due to the low bandwidth of its feedback loop [33,34]. When trying to speed up the AFM acquisition rate, one has to extend the scanner resonance frequency (to avoid excitations) and to increase the AFM electronic feedback bandwidth [39]. Piezotube resonance frequencies are determined to be $300-400 \mathrm{~Hz}$ in XY and $1.3 \mathrm{kHz}$ in $\mathrm{Z}^{1}$, while the lowest resonance frequency is in the order of tens of $\mathrm{kHz}$ for conical or flexure based scanners [38, 40]. Furthermore, AFM electronics have been developed to extend the acquisition rate to several frames per second [35, 188].

In this chapter, the development of an in situ AFM to monitor the oxide growth front under PLD conditions is described. The design of the in situ AFM is based on an AFM flexure scanner, typically used in the field of high-speed AFM (HS-AFM), combined with high bandwidth scanning probe microscopy (SPM) electronics from Leiden Probe Microscopy B.V. [35]. First, the specifications and concept of the instrumentation are described. Then, the instrument design is presented followed by the instrument performance and conclusions regarding the instrument performance.

\footnotetext{
${ }^{1}$ Piezotube resonances of the AFM operating at PLD conditions were analyzed in this work.
} 


\subsection{AFM specifications}

In general, perovskite oxides $\left(\mathrm{ABO}_{3}\right)$ are deposited at pressures ranging from $10^{-6}$ mbar up to 1 mbar $\mathrm{O}_{2}$ and sample temperatures from room temperature (RT) up to around $850^{\circ} \mathrm{C}[17,18,126,127,191]$. Typically, AFM's are not capable of working under these conditions. Piezo's typically operate up to temperatures around its Curie temperature $T_{\mathrm{C}}$ and depolarize at higher temperatures. Often $\mathrm{PZT}^{2}$ is used as piezoelectric material due to their large displacement vs. voltage characteristic and has a $T_{\mathrm{C}}=150{ }^{\circ} \mathrm{C}$. Other type of piezoelectric materials, such as $\mathrm{LiNbO}_{3}$ have a $T_{\mathrm{C}}>150{ }^{\circ} \mathrm{C}$, although they are less suited as the displacement is orders of magnitude lower compared to PZT at the same applied voltage.

A Si cantilever becomes $\pm 90{ }^{\circ} \mathrm{C}$ in close vicinity with a $2 \times 2 \mathrm{~mm}^{2} \mathrm{SrTiO}_{3}$ sample at $\mathrm{T}=750{ }^{\circ} \mathrm{C}$ and $\mathrm{PO}_{2}=10^{-2}$ mbar $\mathrm{O}_{2}$ [33]. Si cantilevers are temperature resistant up to $900{ }^{\circ} \mathrm{C}$ in an oxygen environment, while $\mathrm{Si}_{3} \mathrm{~N}_{4}$ cantilevers deform under these conditions and metal reflex coated cantilevers start to bend due to the difference in thermal expansion coefficient between silicon and metal coating [33]. Besides that, cantilever properties change by an increasing temperature such that it results in a negative frequency shift $\Delta f$.

As $\mathrm{ABO}_{3}$ samples during PLD are heated to elevated temperatures, thermal drift in the system plays an important role to be able to follow growth at the same surface location using AFM. Thermal drift arises from different thermal expansion coefficients of different materials during heating up of the instrumentation. Typically, $0.5 \times 0.5 \mathrm{\mu m}^{2}$ up to $5 \times 5 \mathrm{\mu m}^{2}$ ex situ AFM image sizes are used to visualize $\mathrm{ABO}_{3}$ islands after deposition is stopped [17, 18, 33]. To follow the growth of $\mathrm{ABO}_{3}$ islands on $\mu \mathrm{m}$ length scales over hours, thermal drift should be a small fraction of such typical AFM image sizes. As deposition and growth are separated in time, a sample transfer stage can be applied to transfer a $\mathrm{ABO}_{3}$ sample back and forth in order to prevent hindering of deposition on an as-grown surface. In this case, the sample transfer stage repositioning repeatability should be a small fraction of the AFM image size to visualize / track growth of specific islands. The latter will be more extensively discussed in Ch. 4 .

An AFM scanner operating at PLD pressures should be designed such that an piezo electrical breakdown is prevented. At oxygen PLD pressures of $10^{-6}-1$ mbar $\mathrm{O}_{2}$, the piezo breakdown voltage varies by a change in piezo potential offset. This breakdown voltage is called spark-potential and depends on background gas, background pressure $P$, electrode material and separation distance between electrodes

\footnotetext{
${ }^{2}$ Abbreviation PZT stands for $\mathrm{PbZ}_{1-\mathrm{x}} \mathrm{Ti}_{\mathrm{x}} \mathrm{O}_{3}(0 \leq \mathrm{x} \leq 1)$, which belongs to the perovskite oxide material class.
} 
d. Typically, a voltage range of $330 \mathrm{~V}$ is applied over piezo's in an piezotube AFM scanner. The breakdown voltage of a piezotube is equal to $330 \mathrm{~V}$ having a $P \cdot d$ value of $10^{-4} \mathrm{~cm} \cdot \mathrm{atm}$. For conventional piezotubes, discharge issues at oxide PLD conditions have not been observed [33].

To be able to discriminate individual perovskite oxide $\left(\mathrm{ABO}_{3}\right)$ surface steps, the total noise level should not exceed $0.1 \mathrm{~nm}$, which is a fraction of a typically half or one $\mathrm{ABO}_{3}$ unit step of around $0.2 \mathrm{~nm}$ and $0.4 \mathrm{~nm}$, respectively [19]. Both mechanical and electrical noise contribute to the total AFM noise level. Mechanical noise depends on the vibrational level of the system and the mechanical stiffness between AFM tip and sample surface. The electronic noise level is determined by the noise of several electrical components, such as the AFM feedback electronics, low-noise scan generator, data acquisition system and photodetector. Usually, noise of electrical components increases with bandwidth $f_{\mathrm{bw}}$ proportional to $\sqrt{f_{\mathrm{bw}}}[35,39]$.

Growing $\mathrm{ABO}_{3}$ islands can be monitored when the lateral AFM resolution is smaller than typical lateral $\mathrm{ABO}_{3}$ island sizes during PLD. Lateral $\mathrm{ABO}_{3}$ island sizes have been measured using ex situ AFM after deposition is stopped at the minimum RHEED specular intensity. These studies have found $\mathrm{ABO}_{3}$ lateral island sizes varying from 100 - $500 \mathrm{~nm}[17,18,33]$. However, $\mathrm{ABO}_{3}$ lateral island sizes are expected to be even smaller down to a few unit cells at the early growth stage of a monolayer [193]. The minimum island size that can be measured with AFM is limited by the lateral AFM resolution and on its turn by the AFM tip apex size.

A summary of instrument specifications is described below:

- $\mathrm{O}_{2}$ pressure ranging from $10^{-6}$ mbar up to 1 mbar.

- $\mathrm{ABO}_{3}$ sample temperature ranging from $\mathrm{RT}$ up to $850{ }^{\circ} \mathrm{C}$.

- Vibrational level $<0.1 \mathrm{~nm}$ in $\mathrm{X}, \mathrm{Y}$ and $\mathrm{Z}$ direction.

- Electronic noise floor $<0.1 \mathrm{~nm}$.

- AFM height resolution of $0.1 \mathrm{~nm}$ on perovskite oxide surfaces. Lateral AFM resolution $\approx 10 \mathrm{~nm}$.

- Thermal drift in-plane $\leq 20 \mathrm{~nm} / \mathrm{min}$ and out-of-plane $\leq \pm 700 \mathrm{~nm}$ (Z-range) for one hour AFM imaging.

- $\mathrm{AFM}$ acquisition rate $\geq$ one frame/s $(512 \times 512$ pixels $)$. 


\subsection{Concept}

Fig. 3.1 shows a schematic illustration of a concept of AFM during PLD. In a support frame, an AFM scanner and PLD target are installed and a sample transfer stage is included. The AFM scanner is positioned geometrically separated from the PLD position for a main reason: It prevents blocking of deposition on the asgrown surface and unwanted AFM tip contamination. As deposition and growth in PLD are separated in time, growth can be studied in between subsequent pulses using this concept. A plasma screen between AFM and PLD should prevent any PLD plasma contamination on the AFM scanner.

In this concept, a sample is transferred back and forth between AFM and PLD, where the sample transfer is based on linear motion. A sample heater is mounted on top of a linear motion slider. The concept is such that the side approach can be applied to reduce the time delay $\tau_{\text {approach }}$ between PLD and AFM monitoring of the sample surface, see Ch. 4 [194].

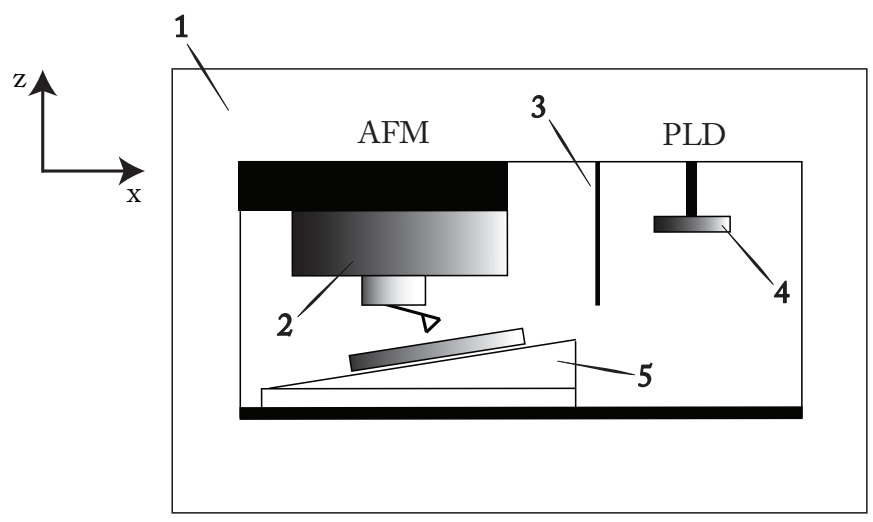

Fig. 3.1 Conceptual drawing of PLD combined with in situ AFM. AFM and PLD are geometrically in position separated to prevent hindering of deposition and tip contamination. A fast transfer stage is moving a sample back and forth between AFM and PLD. 1) support frame, 2) AFM scanner, 3) plasma screen, 4) PLD target and 5) sample/heater transfer stage. 


\subsection{Design}

In this section, the general architecture of the designed in situ AFM in a PLD vacuum setup is described together with a more detailed description of all individual parts.

\subsubsection{PLD chamber and peripheral equipment}

Fig. 3.2 gives a schematic representation of the designed PLD chamber and peripheral equipment. The PLD chamber (dimensions: length $\times$ width $\times$ height; $4600 \times 3950 \times 6900 \mathrm{~mm}^{3}$ ) is a single vacuum system, which differs in shape compared to traditional cylindrical chambers. A low $10^{-7}$ mbar base pressure is reached after a bakeout at $120{ }^{\circ} \mathrm{C}$ for $24 \mathrm{~h}^{3}$. In the designed PLD system ${ }^{4}$, the base pressure is limited by O-rings of the vacuumdoor, valve on entrance flange PLD excimer laser and VAT valve to a vacuum level of $10^{-8}$ mbar. Typical PLD background pressures of $10^{-6}-1$ mbar $\mathrm{O}_{2}$ are measured by almost closing the VAT valve and varying the oxygen flow between $0-100 \mathrm{ml} / \mathrm{min}$. The vacuumchamber is a square-like box design such that a vacuum chamber door is used to open up the system for accessibility to install the AFM-PLD assembly. The vacuumdoor has a DN100CF flange viewport window, revolves by a shaft and can be closed by two closing knobs. Four airpod damping units are placed on an Al profile system ${ }^{5}$.

Matted corks are placed underneath the Al profile system to avoid system resonances $(5 \mathrm{~Hz}, 11 \mathrm{~Hz}$ and $22 \mathrm{~Hz})$ validated by measuring the transfer function in $\mathrm{x}-$ and y-direction according to Segerink et al. [195]. A homebuilt passive damping stage is connected to both the airpods (reduces vibrational level, especially in the z-direction) and vacuumchamber. The PLD chamber houses an Al tube as connection between an AFM-PLD frame and an active damping stage. The active damping stage is mounted as a lid on this PLD chamber. This damping stage ensures good vibration isolation and consists of two parallel Halcyonics MOD2-S active damping control elements (operational from $10-100 \mathrm{~Hz}$ ).

On top of the active damping stage, a five-way cross has been assembled. Two DN100CF flanges with each two sub-D 25 pins electrical feedtroughs have been installed on this cross. These feedtroughs are needed to send and receive signals from and to the AFM scanner, coarse approach steppermotor, slider piezomotor

\footnotetext{
${ }^{3}$ Bakeout can be performed up to $150{ }^{\circ} \mathrm{C}$ and the maximum temperature is limited by the active damping control elements.

${ }^{4}$ A helium leak rate was measured of $3.5 \times 10^{-10} \mathrm{mbar} \cdot \mathrm{l} / \mathrm{s}$ directly after PLD chamber assembly by the factory VDL Enabling Technologies Group Almelo BV.

${ }^{5} \mathrm{Al}$ profiles are ordered from Boikon.
} 


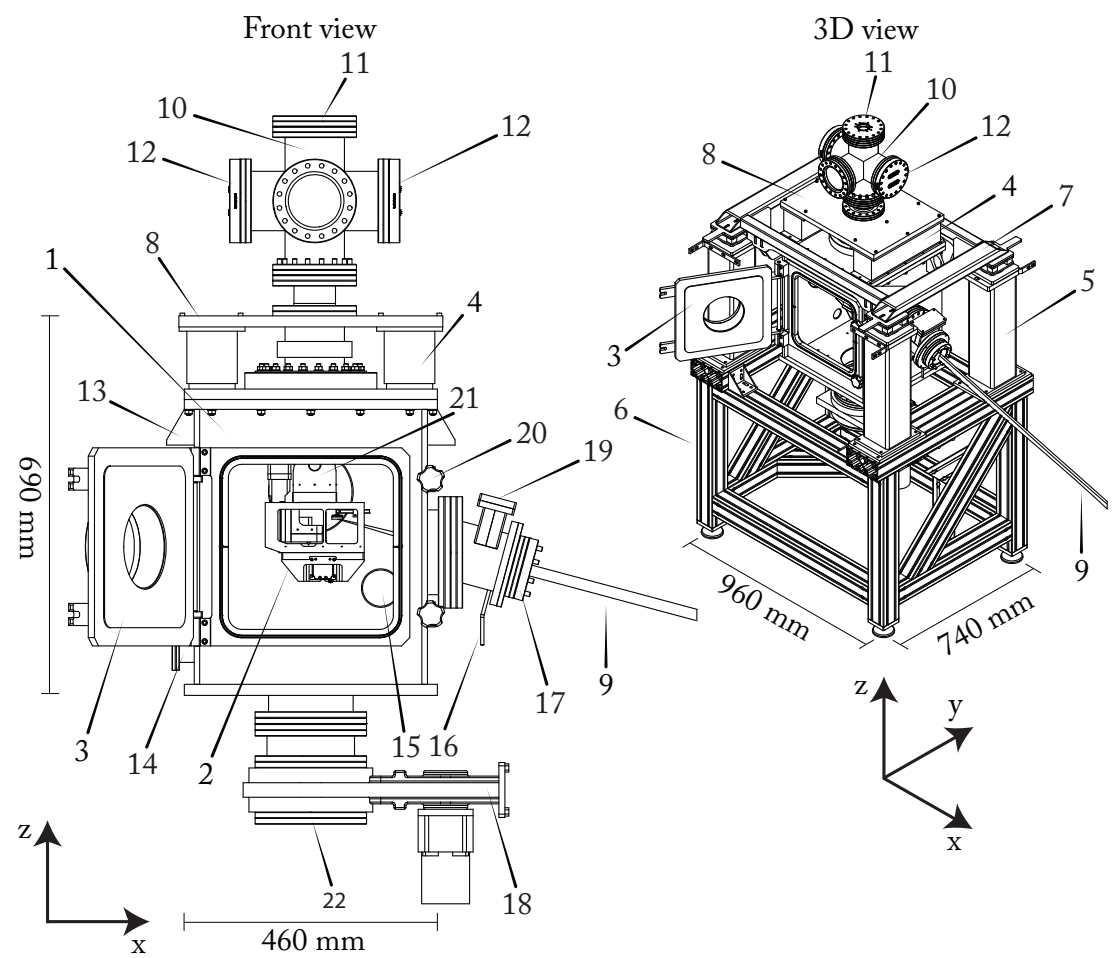

Fig. 3.2 Schematic drawing of PLD vacuum setup and peripheral equipment. 1) PLD vacuum setup, 2) AFM-PLD frame, 3) door PLD chamber, 4) Halcyonics MOD2-S active damping control element, 5) air damped pods, 6) Al profile system, 7) passive damping stage, 8) active damping stage, 9) incoming excimer laser light $\mathrm{KrF}-\mathrm{ArF}$ wavelength $248 \mathrm{~nm}, 10)$ DN100CF five-way cross, 11) fiber feedtrough, 12) electrical feedtrough, 13) mounting unit PLD chamber - passive damping stage, 14) DN40CF flange with Pfeiffer vacuum compact full range pressure sensor $\left(5 \cdot 10^{-9}-1000\right.$ mbar), 15) DN40CF flange reduced to DN16CF with MKS type 626A baratron pressure sensor full-scale pressure/vacuum ranges as low as 0.02 mbar, 16) gasinlet $\mathrm{O}_{2}$, Ar and $\mathrm{N}_{2}, 17$ ) DN63CF flange with quartz glass type 420GSG063, 18) VAT valve with adaptive pressure controller PM$5,19)$ valve to measure laser energy behind window, 20) closing unit door PLD chamber, 21) Al tube which works as cable feedtrough and it connects the active damping stage with the $\mathrm{Al}$ frame, 22) DN200CF flange for a turbopump. The turbo pump is a Pfeiffer turbomolecular pump D-35614 Assler S(N $\left.\mathrm{N}_{2}\right) 60$ l/s with Pfeiffer TC600 controller. 


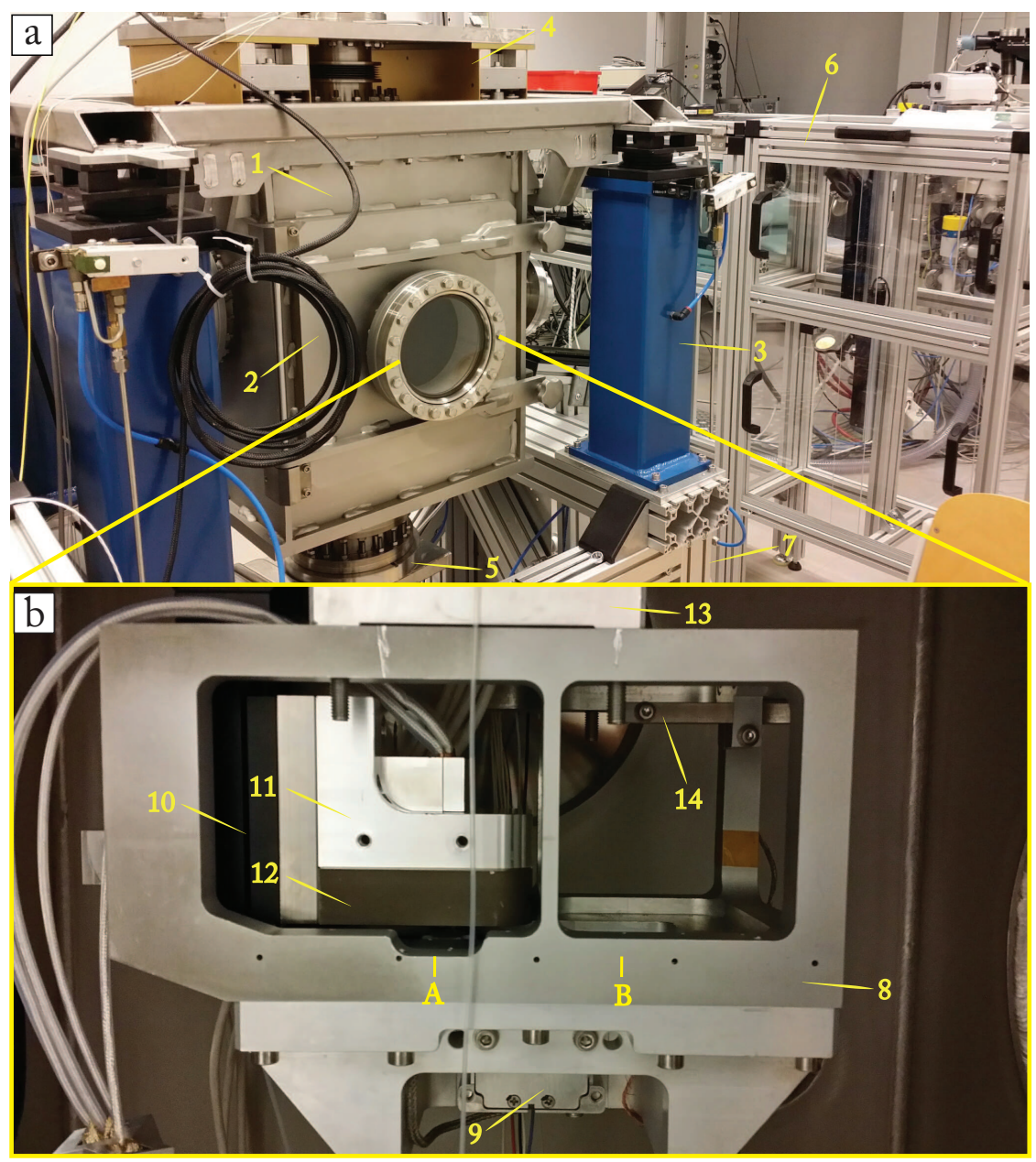

Fig. 3.3 Photographs of a) AFM-PLD setup and b) Al AFM-PLD frame with installed AFM flexure scanner. 1) PLD vacuum setup, 2) PLD chamber door, 3) air damped pods, 4) Halcyonics MOD2-S active damping control element, 5) VAT valve with adaptive pressure controller PM-5, 6) optical rail guiding ( $\mathrm{KrF} \lambda=248 \mathrm{~nm}$ wavelength) laser beam, 7) Al profile system, 8) AFM-PLD Al frame, 9) HR4 vacuum motor, 10) coarse approach stage, 11) mounting bracket, 12) AFM flexure scanner, 13) connection Al tube and the AFM-PLD Al frame, 14) PLD target holder (no PLD target). The AFM position and PLD position are indicated by A and B, respectively. 
drive and readout and heater wires. On top of this cross, an APC-DIN optical feedtrough has been mounted to feed and read the optical signal for detecting the cantilever response through. Three DN40CF flange ports are accessible, where two are used with a DN40CF - DN16CF reducer for a MKS type 626A baratron pressure sensor full-scale pressure/vacuum ranges (capable to measure more accurate typical PLD pressures) and the other is connected with a Pfeiffer vacuum compact full range pressure sensor, where the latter is more accurate at high vacuum pressures $\left(10^{-7}-10^{-3}\right.$ mbar $)$. On the back side of this PLD chamber, there is a DN63CF flange available for optional equipment.

A KrF excimer laser (LXPRO ${ }^{T M}$ from Coherent, Inc) with optical rail is integrated such that a $248 \mathrm{~nm}$ pulsed laser beam with a typical pulse duration of $25 \mathrm{~ns}$ is focused on the PLD target. As an entrance flange for the PLD excimer laser light, a $15^{\circ}$ flange is designed for a $15^{\circ}$ incidence of a laser pulse on the PLD target. The flange is integrated with a quartz glass type 420GSG063 window, a valve for measuring laser intensity behind the window and a gasinlet for $\mathrm{O}_{2}$, Ar and $\mathrm{N}_{2}$ gasses. A gas manifold has been designed to separately let $\mathrm{N}_{2}, \mathrm{O}_{2}$ or Ar into PLD chamber via its gasinlet. Brooks Instruments flow meters type Brooks 5850TR (0 - $100 \mathrm{ml}) \mathrm{O}_{2}$ and Ar are integrated in the manifold to control gas flow of $\mathrm{O}_{2}$ and Ar, respectively.

At the PLD chamber bottom side, a DN200CF flange is connected to a VAT valve typically used for automatic pressure control. On the other side of the VAT valve, a DN200CF - DN63CF reducer has been assembled for connection with a Pfeiffer turbomolecular pump D-35614 Assler S( $\left.\mathrm{N}_{2}\right) 60$ l/s (turbomolecular pump is controlled by a Pfeiffer TC600 controller). Vibrational level characterization is performed using three accelerometers from OCEANO Sensor ${ }^{6}$, type TR1BCN, mounted on three directional fases of a small $30 \times 30 \times 30 \mathrm{~mm}^{3} \mathrm{Al}$ cube, where the latter is screwed onto the $\mathrm{Al}$ support frame. Triggering of PLD laser pulses is controlled by a TSST140103 triggerboard of Twente Solid State Technology (TSST).

\subsubsection{AFM-PLD support frame}

Fig. 3.4, schematically illustrates the AFM-PLD Al frame (frame dimensions: length $\times$ width $\times$ height; $180 \times 100 \times 144 \mathrm{~mm}^{3}$ ). This Al frame, exhibiting a robust and high stiffness design, is attached with an $\mathrm{Al}$ connection tube to an active damping stage. This damping stage has the functionality to lower the vibrational level in the mechanical loop of the AFM. The chosen shape and size of the support

\footnotetext{
${ }^{6}$ Oceana Sensor, 1632 Corporate Landing Parkway, Virgina Beach, VA 23454.
} 


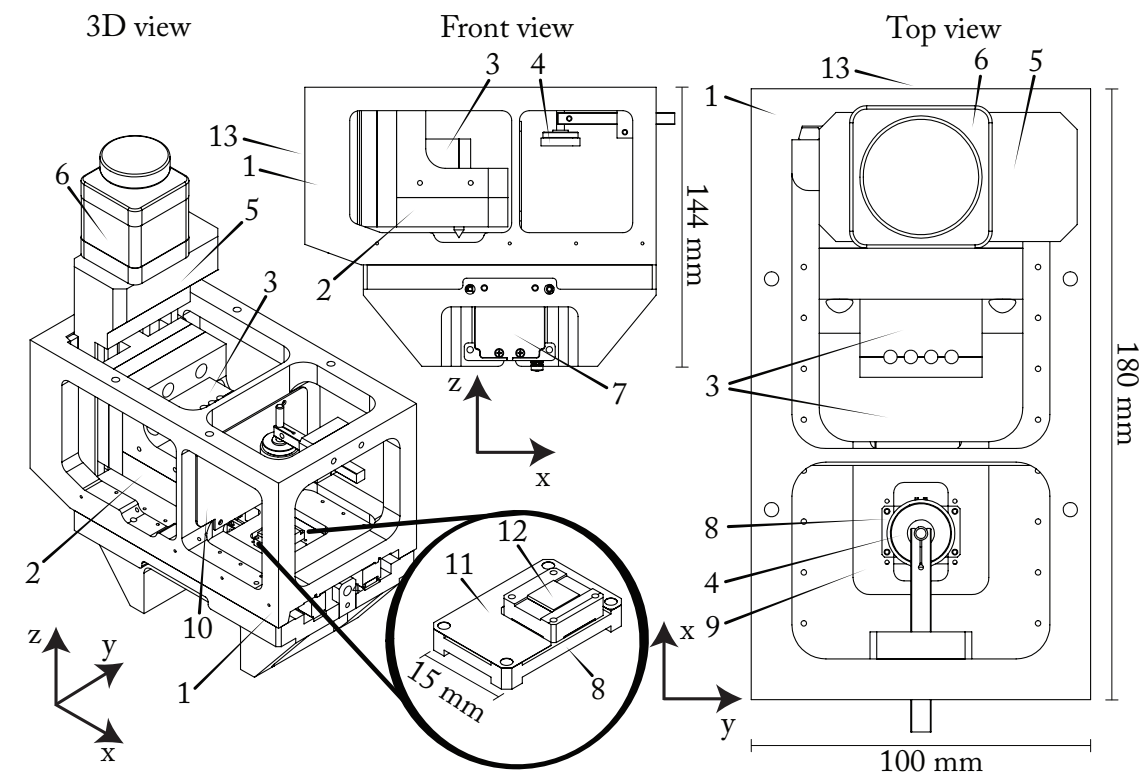

Fig. 3.4 Schematic drawing of support frame including AFM and PLD. 1) Al frame, 2) AFM flexure scanner, 3) mounting bracket, 4) PLD target 5) coarse approach stage, 6) Vexta 5 phase stepper motor PK545-B, 7) HR4 nanomotion ultrasonic piezomotor, 8) macor heater stage, 9) sample transfer stage, 10) plasma screen, 11) two Kamet HDA pt200 RTD's connected in serie, 12) $5 \times 5 \mathrm{~mm}^{2}$ sample on top of an $5 \times 5 \mathrm{~mm}^{2}$ Pt plate and 13) location of screwed cube for integration of acceleration level sensors.

frame is based on required sizes and shape of the AFM scanner, PLD plasma and a mechanical stiffness such that the required vibrational level is not exceeded. The Al frame consist of two assembled Al frames, where the top frame is used for integration of both the AFM scanner and PLD target. The bottom frame has the functionality to integrate a sample transfer stage enabling back and forth sample transfer between AFM and PLD.

A HR4 nanomotion ultrasonic vacuum piezomotor with low vibrational level, a repositioning resolution within $20 \mathrm{~nm}$ and a maximum speed of $244 \mathrm{~mm} / \mathrm{s}$ is installed in the bottom frame in order to propel the sample transfer stage. The bottom frame contains an $94 \mathrm{~g} \mathrm{Al}$ stage to ensure low mass for sample transfer and therefore fast acceleration. Two strips are placed on the bottom side of the Al stage with vacuum-compatible glue, where one ceramic strip is used as piezomotor friction bar and a Renishaw RGS-20 gold strip for position readout by a Renishaw RGH25F UHV optical encoder (resolution of $5 \mathrm{~nm}$ ). A heater can be mounted on top of the Al stage (using four M1.6 screws). 
An Owis LM60 linear coarse approach stage with on top a Vexta 5 phase PK545B stepper motor (having a step resolution of $100 \mathrm{~nm} / \mathrm{step}$ ) is screwed in the top frame. A homebuilt Al mounting bracket has been designed as connection unit between coarse approach and AFM scanner. The PLD target holder is made out of $\mathrm{Al}$ with two screws to adjust its position. The target can be adjusted in height and position along the sample transfer direction. In the direction perpendicular to the sample transfer, the target position is fixed. In this design, the distance between the AFM tip and PLD target center is $57 \mathrm{~mm}$ (distance center PLD target to plasmascreen is $25 \mathrm{~mm}$ ). During sample and cantilever replacement, the Al plasma screen has to be removed to unscrew and uninstall the AFM flexure scanner with mounting bracket. A plasma screen can be mounted to prevent plasma contamination onto the AFM flexure scanner.

\subsubsection{AFM flexure scanner}

The AFM scanner is based on a flexure, see Fig. 3.5, which is often used in the field of HS-AFM [62]. Flexure scanners are used as an alternative to commonly used low resonance frequency piezotubes to extend the mechanical scanner bandwidth from $\mathrm{Hz}$ to the $\mathrm{kHz}$ range [40]. In addition, stacked piezo's integrated in a flexure results in less cross-coupling, hysteresis and creep as compared to generally used piezotubes. The AFM flexure in this work has been made out of Ti6Al4V to achieve high stiffness, high resonance frequency and low thermal expansion in order to minimize thermal drift. A calculated lowest resonance of the designed flexure scanner is found of $\approx 19 \mathrm{kHz}$ in XY-direction ${ }^{7}$. The maximum achievable linerate is therefore between $0.19-1.9 \mathrm{kHz}$ based on this scanner design. This has been calculated according to $1 / 100$ th $-1 / 10$ th of its lowest resonance frequency [40].

Double XY stacked PI PL-088.30 piezo actuators from PI company are used to achieve the required scan range. Standard scan range of these piezo's is $4 \mu \mathrm{m}$, but strongly reduces to $1.4 \mathrm{\mu m}$ due to a high preload. The Z-flexure is aligned nearly in the center of XY-flexure and can be positioned by the double stacked XYactuators in the XY-plane. For Z-actuation and sensing, a PI PD080.30 piezoring is located underneath the cantilever stage with $1.4 \mu \mathrm{m}$ effective scan range. A counterbalance PI PD080.30 piezo has been integrated in the Z-flexure to counter momentum generated by center of mass movement of the Z-piezo. The whole cantilever unit is displaced by this Z-piezo such that the tip on cantilever chip is effectively moving towards sample surface. This cantilever unit exists of two parts. The bottom part is screwed on top of Z-flexure and is milled under $7^{\circ}$. $\mathrm{A} \mathrm{ZrO}_{2}$ ferrule $(\oslash=125 \mu \mathrm{m}$ cladding together with a $\oslash=9 \mu \mathrm{m}$ fiber inside) is glued into

\footnotetext{
${ }^{7}$ Simulated using finite element analysis (FEA) with Autodesk ${ }^{\circledR}$ Invertor ${ }^{\circledR}$.
} 


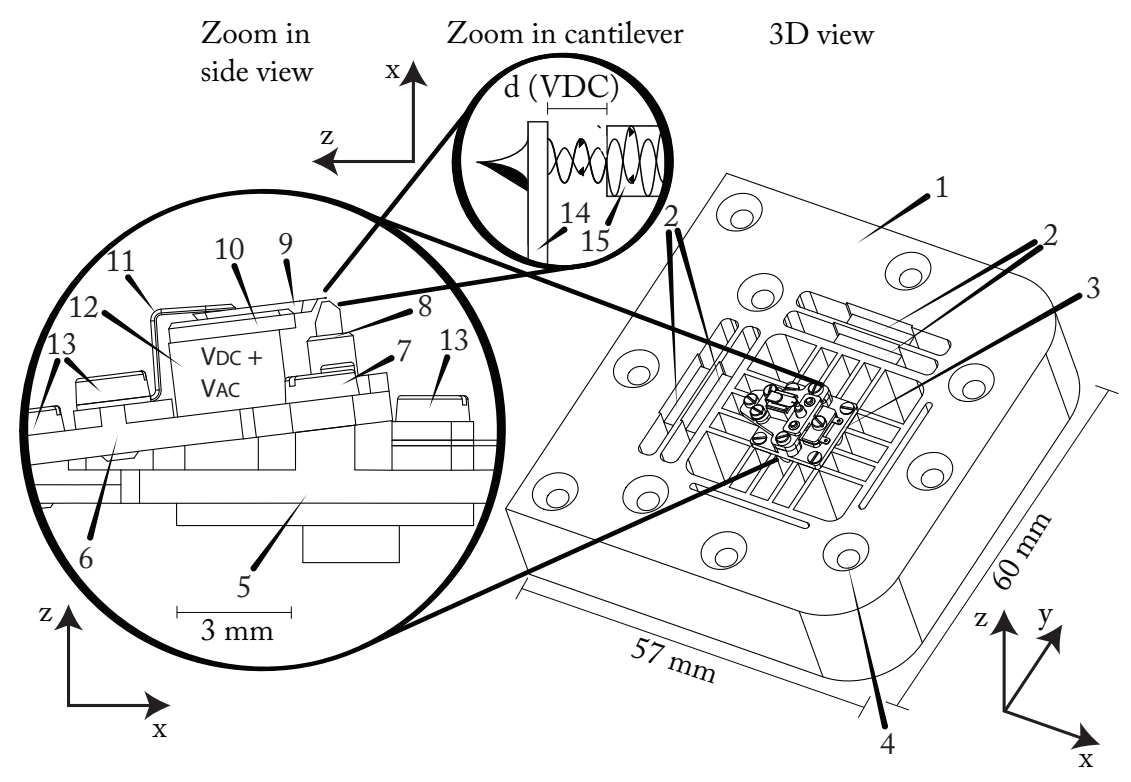

Fig. 3.5 Schematic drawing of high resonance frequency AFM flexure scanner 1) Ti6Al4V flexure, 2) stacked XY actuators PI PL-088.30 piezo's, 3) cantilever stage, 4) screw holes, $5)$ connection plate between cantilever base plate and flexure, 6) cantilever base plate $7^{\circ}$ angle with respect to connection base plate 7) CuBe electrical contacts dither piezo, 8) $\mathrm{ZrO}_{2}$ ferrule with $125 \mu \mathrm{m}$ fiber cladding inside, 9) cantilever chip, 10) chip holder, 11) cantilever clamping spring, 12) dither piezo - PI PL033.30 piezo stack, 13) M1 screws, 14) cantilever, 15) optical fiber end. Distance between fiber end and cantilever $\approx 40-50 \mu \mathrm{m}$, where the optimal interference working point distance $\mathrm{d}\left(V_{\mathrm{DC}}\right)$ in the cavity can be tuned with a DC voltage $V_{\mathrm{DC}}$ applied to the dither piezo.

the small hole in the bottom part. On top of the bottom part of the cantilever stage, a top part unit is placed existing of a flat plate with on its top a PI PL033.30 piezo stack (dither piezo), cantilever spring and $\mathrm{CuBe}$ dither piezo contacts.

Optical interference is used instead of optical beam deflection to detect cantilever modulating properties. Beam deflection requires use and adjustment of optical instrumentation, such as mirrors and is unpractical under PLD conditions, where space is lacking. Therefore, usage of interferometric detection results in a more compact design. A laser beam (wavelength $\lambda=790 \mathrm{~nm}$, interference period $\lambda / 2$ $=395 \mathrm{~nm}$ ) is reflected from the cantilever top plane and fiber-air interface, where the cantilever and fiber are separated by $\approx 40-50 \mu \mathrm{m}$. A reference wave is reflected by the planar end of the fiber (glass-air interface). The detected wave is reflected by the top face of the cantilever. Both light waves interfere on an $400 \mathrm{kHz}$ photodiode after both are transmitted through a fiber into the Surface Imaging Systems 


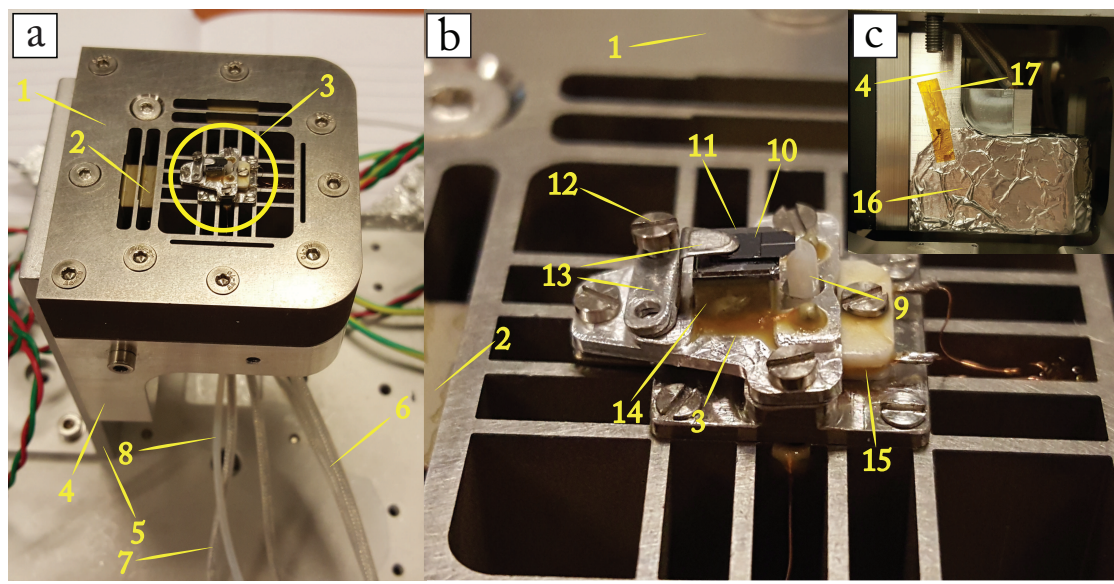

Fig. 3.6 Photographs of a) AFM flexure scanner, b) a zoom-in of AFM flexure scanner and of c) AFM flexure scanner with Al foil radiation shield. 1) AFM flexure scanner, 2) stacked XY actuators PI PL-0.88.30 piezo's, 3) cantilever stage, 4) mounting bracket, 5) AFM flexure holder unit to exchange cantilever, 6) cables $\mathrm{X}, \mathrm{Y}$ and $\mathrm{Z}$ piezo actuators, 7) cable dither piezo, 8) fiber protective housing, 9) ferrule, 10) cantilever chip, 11) cantilever alignment chip, 12) M1 screw cantilever spring, 13) cantilever spring, 14) dither PI PL033.30 piezo stack actuator, 15) CuBe electrical contacts dither piezo, 16) radiation shield of $\mathrm{Al}$ foil, 17) kapton tape.

$\mathrm{GmbH}$ (SIS) electronics ${ }^{8}$. A 10/90 splitter has a functionality to compensate large intensity differences between $\mathrm{a} \approx 96 \%$ reference and $\approx 4 \%$ detection intensity. This interferometer signal depends on the phase difference $\Delta \varphi_{\text {int }}$ caused by optical path length difference $d\left(V_{\text {DC }}\right)$ between a reference and a detected wave. If $A_{\text {int }}$ and $B_{\text {int }}$ are amplitudes of a reflected and detected wave $\mathbf{A}$ and $\mathbf{B}$ and $\Delta \varphi_{\text {int }}$ is their phase difference equal to $\frac{4 \pi}{\lambda}\left(d_{V_{\mathrm{DC}}}+d_{V_{\mathrm{AC}}}\right)$, then they add up by superposition to a total intensity (I) according to Eq. 3.1:

$$
I \sim|\mathbf{A}+\mathbf{B}|^{2}=A_{\mathrm{int}}^{2}+B_{\mathrm{int}}^{2}+2 \cdot A_{\mathrm{int}} \cdot B_{\mathrm{int}} \cdot \cos \Delta \varphi_{\mathrm{int}}
$$

The optimal interference working point distance of the cavity can be adjusted by the phase difference $\Delta \varphi_{\text {int }}$ between reference and detection wave. Tuning of $\Delta \varphi_{\text {int }}$ is done by applying a DC voltage $\left(V_{\mathrm{DC}}=5.9-59.6 \mathrm{~V}\right)$ over the dither piezo $($ measured piezoelectric coefficient $=11.8 \mathrm{~nm} / \mathrm{V})$ to regulate the distance $d\left(V_{\mathrm{DC}}\right)$ between the cantilever top face and fiber end. On top of $V_{\mathrm{DC}}$, an AC voltage

\footnotetext{
${ }^{8}$ Company is acquired by Bruker.
} 
(typically $V_{\mathrm{AC}}=1-1000 \mathrm{mV}$ ) is applied to oscillate a cantilever at its resonance frequency $f_{0}$ in dynamic mode AFM.

\subsubsection{AFM electronics}

Two AFM scanning modes are demonstrated namely Tapping-Mode (TM) also called Amplitude-Modulation (AM) and true Non-Contact (NC) mode also known as Frequency-Modulation (FM). There is a main difference between both AFM scanning modes. During TM-AFM, the cantilever amplitude $A_{\text {osc }}$ is feed into the AFM height feedback, while during FM-AFM the frequency shift $\Delta f$ is used for height feedback. Typically, $A_{\text {osc }}$ is measured by taking an RMS $A_{\text {osc }}$ of an modulating wave signal, where $\Delta f$ is measured using a phase locked loop (PLL) demodulator $[62,157]$. Most electronic AFM components are applicable for both AFM scanning modes except the components for either measuring $A_{\text {osc }}$ or $\Delta f$. Here, $A_{\text {osc }}$ is detected from a modulating interferometer signal using a $10 \mathrm{MHz}$ bandwidth peak / phase detector described by Ando et al. [39] and $\Delta f$ is detected from a modulating interferometer signal using an $50 \mathrm{kHz}$ Zurich Instruments (ZI) PLL demodulator.

An SIS electronics box is adapted as detection optics / electronics for measuring the modulating cantilever properties during AFM (originally, this AFM electronics was used as electronics for scanning, interferometric detection and topographical height feedback). Here, the SIS electronics has two functionalities, feedback on the (optimal) interference working point by controlling $V_{\mathrm{DC}}$ and the modulating cantilever signal is acquired (from the BNC interferometer out).

The interferometer out is integrated in an electrical circuit such that the properties $A_{\text {osc }}$ or $\Delta f$ from the cantilever can be detected. After measuring $A_{\text {osc }}$ or $\Delta f$, the signal is used as input for topographic feedback $(0-10 \mathrm{~V})$ by the AFM electronics. Scanning and topographic feedback is performed using commercial available highspeed SPM electronics from Leiden Probe Microscopy B.V. ${ }^{9}$ [35]. The FM-AFM mode in this work is in Constant-Excitation (CE) FM-AFM as there is only a feedback used on $\Delta f[196]$.

In both AFM-modes, a cantilever is driven at its $f_{0}=140-190 \mathrm{kHz}$ with an $A_{\text {osc }}=5-120 \mathrm{~nm}$ (calibrated by the SIS interferometer) using a signal generator of a ZI HF2LI. A laser beam $(\lambda=790 \mathrm{~nm})$ from a laser inside the SIS interferometer is emitted to the cantilever. Its reflection from the surface and from the glassfiber interface causes interference, where the interference intensity is detected by a $400 \mathrm{kHz}$ bandwidth photodetector. A photodetector noisefloor of $38 \frac{\mathrm{fm}}{\sqrt{\mathrm{Hz}}}$ is

\footnotetext{
${ }^{9}$ Video-rate SPM controller
} 
determined from frequency analyzer measurements.

\subsubsection{Heater}

Different type of heaters i.e. resistive, radiative and laser heating have been applied during PLD of oxides with each their own advantage. Commonly used PLD heaters have a main disadvantage, namely a relative large input power (tens of Watts) is needed to heat an $5 \times 5 \mathrm{~mm}^{2} \mathrm{ABO}_{3}$ sample to PLD temperatures of $600-850{ }^{\circ} \mathrm{C}$. This results in a large heat radiation power and therefore warming of both the cantilever and AFM scanner. Besides that, thermal heat loss has to be minimized in order to extend the sample temperature avoiding scanner piezo depolarization [33].

Several research groups developed low input power heater stages to extend the sample temperature during SPM [197, 198, 199]. Potentially, these heaters are suitable for heating $\mathrm{ABO}_{3}$ samples up to elevated temperatures, but many have a disadvantage crucial for a specific experimental purpose. For example, direct current heaters are not applicable as most $\mathrm{ABO}_{3}$ substrates are insulators. Commercial available heaters, such as Boralectric heating elements are not designed for heating $5 \times 5 \mathrm{~mm}^{2}$ samples as they are only applied to much larger sample area's and rarely custom made.

A PLD heater for $2 \times 2 \mathrm{~mm}^{2}$ samples with an input power of below 2 Watts under metal-oxide PLD conditions has been reported [34]. However, this high temperature stage is only applicable for sample sizes $\leq 2 \times 2 \mathrm{~mm}^{2}$, where $5 \times 5 \mathrm{~mm}^{2} \mathrm{ABO}_{3}$ samples are commonly used in materials science laboratories. A homebuilt heater has been designed to heat $5 \times 5 \mathrm{~mm}^{2} \mathrm{ABO}_{3}$ samples up to typical PLD temperatures, see Fig. 3.7. In addition, here it has been proven that the designed heater is sufficient rigid.

Here, macor has been used as thermal insulator (thermal conductivity $\lambda_{\mathrm{c}}=1.46$ $\mathrm{W} / \mathrm{m} \cdot \mathrm{K}$ ) to reduce thermal loss by heat conduction to the transfer stage and therefore resulting in heating up of the support frame. Furthermore, macor has a thermal expansion coefficient $\alpha_{\text {lin }}=1.23 \times 10^{-7} \mathrm{~K}^{-1}$ and can operate up to a maximum temperature $T_{\max }=1000{ }^{\circ} \mathrm{C}$ (continuous at $800{ }^{\circ} \mathrm{C}$ ). In spite of its high thermal emission coefficient $\epsilon \approx 0.9$, the thermal radiation is low as the temperature of the macor piece is expected to be significantly lower than the sample temperature.

A custom designed Mo spring is placed on top of the bottom macor part. This spring clamps two Kamet HDA pt200's to an $5 \times 5 \mathrm{~mm}^{2} \mathrm{Pt}$ sample plate. This Pt sample plate fits exactly in the top macor part. Four screws are used to clamp the 

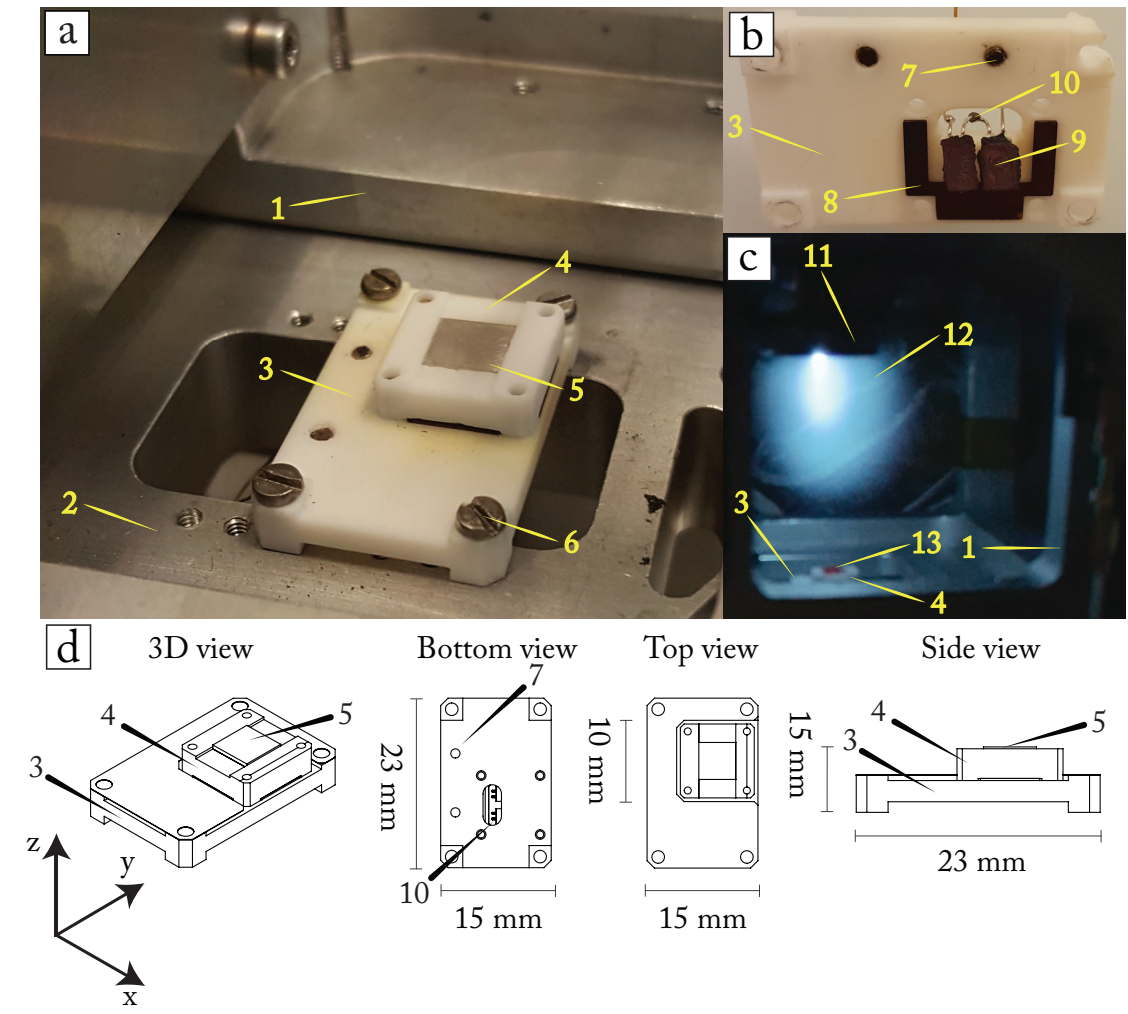

Side view

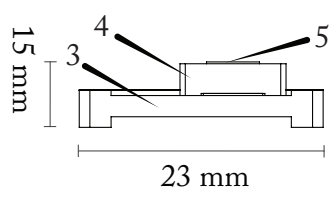

Fig. 3.7 Photographs of designed heater a) mounted on transfer stage, b) without macor top part, c) during $\mathrm{BiFeO}_{3}$ deposition at $T=650{ }^{\circ} \mathrm{C}$ and d) schematic drawing. 1) $\mathrm{Al}$ support frame, 2) transfer stage, 3) macor bottom part heater, 4) macor top part heater (top face shaved off $\left.\alpha=1^{\circ}, 5\right) 5 \times 5 \mathrm{~mm}^{2}$ Pt plate, 6) M1.6 screw, 7) screw to connect electrical wires, 8) molybedum spring for clamping pt-200 heater elements, 9) Pt paint

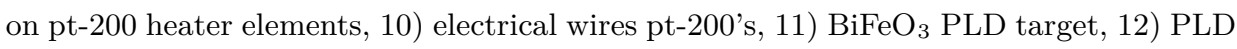
plasma, 13) heated $\mathrm{SrTiO}_{3}(001)$ substrate with as-grown $\mathrm{BiFeO}_{3}$ film on top.

top macor part on the bottom macor part. By screwing this top macor part on the bottom macor part, a force is load on the Mo spring to clamp the pt-200's. The two Kamet HDA pt200's are connected in serial by using spot welding to reduce contact resistance.

A Delta ES 150-0300-0.45 is used as power supply and current read-out. To measure the serial pt-200's resistance, a Fluke 115 multimeter is placed in parallel to measure ohmic resistance over two serial pt-200's. Calibration of the sample temperature was done by measuring the sample plate with another pt-200 thermal resistor. The pt-200 temperature accuracy is $\pm 15{ }^{\circ} \mathrm{C}$ and its temperature is 
constant over time. Thermally conducting $\mathrm{Pt}_{\text {paint }}{ }^{10}$ (melting point $>850{ }^{\circ} \mathrm{C}$ ) is used instead of commonly used $\mathrm{Ag}$ paint due to the low sublimation temperature of $\mathrm{Ag} \approx 500{ }^{\circ} \mathrm{C}$ in vacuum [33]. Furthermore, the Pt paint improves the temperature homogeneity and has a lower temperature gradient between heating elements and $\mathrm{ABO}_{3}$ substrate.

\subsection{Performance}

The most important aspects of the AFM-PLD setup are presented. After demonstrating the vibrational level and PLD characteristics, the AFM imaging performance is demonstrated by imaging a $\mathrm{SrTiO}_{3}(001)$ substrate under different conditions, such as under vacuum and typical PLD conditions. Furthermore, the AFM imaging performance is demonstrated on a $\mathrm{BiFeO}_{3}$ film deposited on $\mathrm{SrTiO}_{3}(001)$ under its PLD growth conditions.

\subsubsection{Vibrational level and stage translation}

Fig. 3.8 shows the $\mathrm{X}, \mathrm{Y}, \mathrm{Z}$ acceleration levels of the AFM-PLD setup operating at $\mathrm{PO}_{2}=0.1$ mbar $\mathrm{O}_{2}$ and acceleration levels of a commercial AFM setup ${ }^{11}$ operating in air. Both systems are placed on a VC-G classified floor ${ }^{12}[195,200]$. The vibrational level sensors are positioned in the mechanical loop from tip to the sample, see Fig. 3.4. The passive damping system suppresses the higher frequencies $\geq 100 \mathrm{~Hz}$, whereas the active damping stage significantly decreases acceleration levels from $10-100 \mathrm{~Hz}$. Damping of the system by the airpods is mainly done in the Z-direction. At $\mathrm{PO}_{2}=0.1$ mbar $\mathrm{O}_{2}$, the pre- and turbopump, VAT Valve and mass flow controllers are running, but no significant increase in acceleration levels is observed. Acceleration levels of the AFM-PLD setup are similar to the commercial AFM, where in the AFM-PLD setup some system resonances are visible in the frequency range from $100-500 \mathrm{~Hz}$. Resonance peaks of 11, 22 and $32 \mathrm{~Hz}$ are only appearing in the frequency spectrum without active damping. Resonance frequencies of the Al support frame are found at 140, 190 and several peaks between $300-400 \mathrm{~Hz}$. These resonance peaks are indicated by arrows in Fig. 3.8.

During stage transfer experiments, the time domain signal was recorded in parallel to acceleration levels measurements. The vibrational decay time $\tau_{\text {delay }}$ of the me-

\footnotetext{
${ }^{10}$ SPI platinum paint, SPI supplies division of structure probe, Inc. P.O. Box 656, West Chester, PA 19381-0656 USA.

${ }^{11}$ Bruker Dimension Icon.

${ }^{12}$ Vibration criteria (VC) curves are a definition of floor vibrations to meet the required vibrational level of equipment.
} 

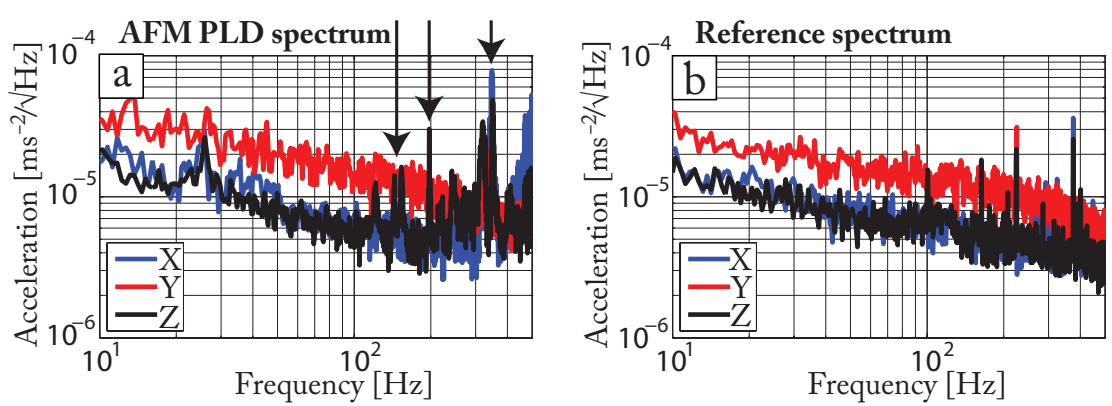

Fig. 3.8 Vibration spectra by measuring the acceleration levels of the, a) AFM-PLD setup at a typical oxygen PLD pressure of $10^{-1}$ mbar $\mathrm{O}_{2}$ and active damping halcyonics control elements turned on (system placed on a VC-G floor) and of a b) Bruker Dimension Icon AFM as reference system (specification AFM height resolution $<0.1 \mathrm{~nm}$ ). The peaks at $\approx 140,190 \mathrm{~Hz}$ and between $300-400 \mathrm{~Hz}$ (indicated by the arrows) are resonance frequencies of the $\mathrm{Al}$ support frame.

chanical AFM tip-sample loop is determined from the time domain signal from the moment that the transfer stage velocity is zero at the AFM position after sample transfer from PLD to AFM, for more details see Ch. 4. Using active damping, a $\tau_{\text {delay }}$ time is found of $0.4 \mathrm{~s}$, while without active damping $\tau_{\text {delay }}=1.0 \mathrm{~s}$. In the parallel acceleration level measurements, resonance peaks are observed at 140 and $280 \mathrm{~Hz}$ directly after stage deceleration. Back and forth sample transfer between AFM and PLD is possible in $0.5 \mathrm{~s}$ at the HR4 motor maximum acceleration and velocity.

Some unwanted resonance peaks were observed in the $\mathrm{X}, \mathrm{Y}$ transfer functions of the passive damping stage [195]. The X- and Y-acceleration transfer function showed several low resonance frequencies at 5, 11 and $22 \mathrm{~Hz}$. These harmonic $\mathrm{X}, \mathrm{Y}$ passive stage resonances are damped such that the acceleration levels were decreased by almost an order of magnitude by placing matted corks underneath the Al profile frame (initially, Al profile legs were used) ${ }^{13}$. Furthermore, four small Viton 009 O-rings are placed between the Al support frame and Al tube to strongly damp a resonance at $140 \mathrm{~Hz}$. Without Viton 009 O-rings, the resonance peak is appearing in the frequency spectrum only when active damping is utilized. During AFM measurements, the coarse approach steppermotor is switched off to prevent resonances at $50 \mathrm{~Hz}$ and its harmonic modes degrading the AFM performance.

13 The acceleration levels are decreased to $\approx 10^{-5} \frac{m}{s^{2} \sqrt{\mathrm{Hz}}}$ using active damping control units. Here, the acceleration levels were measured at $\mathrm{PO}_{2}=10^{-1}$ mbar $\mathrm{O}_{2}$, which is a typical PLD oxygen background pressure to deposit $\mathrm{ABO}_{3}$ thin films. 


\subsubsection{AFM - PLD characteristics}

The cantilever resonance frequency $\mathrm{f}_{0}$ and the cantilever quality $Q_{\text {c }}$ factor are nearly constant at pressures ranging from $10^{-6}-0.1$ mbar $\mathrm{O}_{2}$ [201]. However, $Q_{\mathrm{c}}$ increases from a few hundred to tens of thousands in the pressure regime 1 bar 0.1 mbar and therefore the FWHM of the amplitude vs frequency curve decreases strongly $\left(Q_{\mathrm{c}}=f_{0} / \mathrm{FWHM}\right)[33]$. Another influence on drift in $f_{0}$ is a change in cantilever temperature. AFM on a sample at elevated temperatures results in warming of the cantilever. The cantilever temperature increase results in a negative frequency shift as the cantilever expands and the Young's modulus of a silicon cantilever (without coating) decreases with temperature as:

$$
\Delta T_{\mathrm{c}}=\frac{2 \Delta f}{f_{0}\left(\alpha_{\text {lin }}-\alpha_{\mathrm{E}}\right)}
$$

where $\Delta T_{\mathrm{c}}$ is the temperature shift of the cantilever, $\Delta \mathrm{f}$ the cantilever resonance frequency shift in $\mathrm{Hz}, \mathrm{f}_{0}$ the cantilever resonance frequency in $\mathrm{Hz}, \alpha_{\text {lin }}$ the linear thermal expansion coefficient $\frac{p p m}{K}$ and $\alpha_{E}$ youngs modulus in $\frac{p p m}{K}$. According to equation 3.2 , the calculated frequency shift temperature coefficient of the used silicon cantilever is $-4.6 \mathrm{~Hz} / \mathrm{K}$. Here a $\Delta f=200 \mathrm{~Hz}$ is measured resulting in a cantilever temperature $\approx 70{ }^{\circ} \mathrm{C}\left(\approx \mathrm{RT}+\Delta T_{\mathrm{c}}\right)$ at a sample temperature $T=600{ }^{\circ} \mathrm{C}$ and $\mathrm{PO}_{2}=10^{-1}$ mbar $\mathrm{O}_{2}$.

Moreover, sample heating results in a cantilever stage temperature increase due to thermal radiation of the heater. This temperature increase affects the optimal interference working point distance $d\left(V_{\mathrm{DC}}\right)$ of the cavity by the different thermal expansion rates of the different materials. The expansion is in the order of several interference fringes (several hundreds of nanometers) under typical PLD conditions. This drift can be reduced by preventing warming up of the AFM flexure scanner. At $T=600{ }^{\circ} \mathrm{C}$, thermal radiation $\left(\sim T^{4}\right)$ dominates causing a temperature increase of the whole AFM scanner. In this work, a piece of Al foil (thermal emissivity $\epsilon=0.04$ ) was placed around the AFM scanner as radiation shield (see Fig. $3.6 \mathrm{c}$ )). After placing a radiation shield around the AFM scanner, the DC drift strongly decreased without exceeding a single interference fringe during warming of $\mathrm{SrTiO}_{3}(001)$ from RT up to $600{ }^{\circ} \mathrm{C}$ at a background pressure of $\mathrm{PO}_{2}=10^{-1}$ mbar $\mathrm{O}_{2}$.

Fig. 3.9 a) shows the heater input power versus sample temperature of a $\mathrm{SrTiO}_{3}(001)$ substrate measured in various oxygen pressures. The difference in required heater input power is negligible to reach the same sample temperature in the pressure regime $\mathrm{PO}_{2}=10^{-2}$ mbar $\mathrm{O}_{2}-10^{-6} \mathrm{mbar}_{2}$, whereas at $\mathrm{PO}_{2}=10^{-1}$ mbar $\mathrm{O}_{2}$ the 

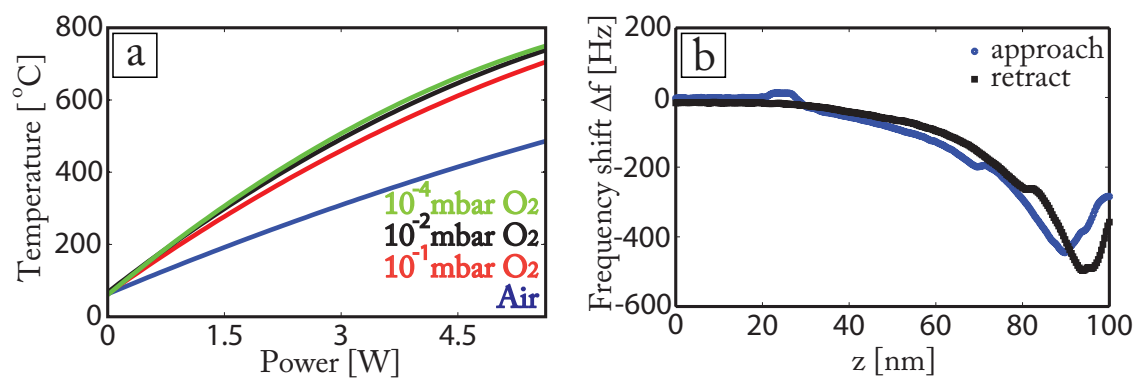

Fig. 3.9 Heater characteristics and force-distance curve. a) Input power vs. sample temperature in different pressures using an $5 \times 5 \mathrm{~mm}^{2} \quad \mathrm{SrTiO}_{3}(001)$ substrate, b) force-distance curve between $\mathrm{AFM}$ tip and $\mathrm{SrTiO}_{3}(001)$ substrate at RT and $\mathrm{PO}_{2}=10^{-1}$ mbar $\mathrm{O}_{2}$. Free oscillation amplitude is $120 \mathrm{~nm}$ of SSS Si cantilever (force spectroscopy can be used to study neck formation between tip and sample under PLD conditions [33]).

heater input power $(>1 \mathrm{~W})$ to reach the same sample temperature $\left(T>200{ }^{\circ} \mathrm{C}\right)$ starts to deviate significantly. Increasing the pressure up to 1 bar results in a much larger required input power to heat a sample to the same temperature compared to vacuum conditions. For example, a sample temperature of $400{ }^{\circ} \mathrm{C}$ requires a heater input power of $2.4,2.6$ and $4.5 \mathrm{~W}$ at $10^{-2}$ mbar $\mathrm{O}_{2}, 10^{-1} \mathrm{mbar}_{2}$ and in air, respectively. At $10^{-1}$ mbar $\mathrm{O}_{2}-10^{-6}$ mbar $\mathrm{O}_{2}$, the thermal heat loss contribution of convection is small [33]. The difference in heater input power (and heat loss) is therefore contributed by a change in convection behavior at 1 atm $-10^{-2}$ mbar $\mathrm{O}_{2}$. The temperature gradient of $150{ }^{\circ} \mathrm{C}$ between pt-200's and the $\mathrm{SrTiO}_{3}(001)$ sample at $10^{-2}$ mbar $\mathrm{O}_{2}$ to $10^{-6}$ mbar $\mathrm{O}_{2}$ at PLD temperatures. The same temperature gradient is measured between Pt200 elements and a $\mathrm{SrTiO}_{3}(001)$ sample at $10^{-1} \mathrm{mbar}_{2}$ and $\mathrm{T}=600{ }^{\circ} \mathrm{C}$. Therefore, the maximum reachable $\mathrm{SrTiO}_{3}(001)$ substrate temperature is limited to $700{ }^{\circ} \mathrm{C}$ due to the temperature gradient of $150{ }^{\circ} \mathrm{C}$. From these experiments, it was found that the serial pt-200's gets a breakdown exceeding a resistance $R_{2 \mathrm{pt} 200}=1600 \Omega$ corresponding to an input power of $6.0 \mathrm{~W}$, which limit the maximum pt-200 temperature. When the AFM-PLD setup is temperature stabilized after 1.5 - $2 \mathrm{~h}$ with a sample temperature of $T=600{ }^{\circ} \mathrm{C}$ at $10^{-1}$ mbar $\mathrm{O}_{2}$, the scanner and support frame temperature $\approx 30-40{ }^{\circ} \mathrm{C}$, which is below the HR4 maximum operating temperature of $50{ }^{\circ} \mathrm{C}^{14}$.

An issue at elevated sample temperatures is neck formation between AFM tip

\footnotetext{
${ }^{14}$ Specs are only given for ambient.
} 
and sample surface [33, 159]. Previously, force spectroscopy has been used as a method to study neck formation [33]. Qualitative force-distance curves can be made as $\Delta f$ is proportional to the interaction force $F_{\mathrm{ts}}$ between AFM tip and $\mathrm{ABO}_{3}$ sample $[155,157]$. Here, $\Delta f$ from the HF2LI auxiliary output is fed into a LPM controller channel for read-out and the X-generator signal is connected to the Z-driver (a force-distance curve can be plotted by taking a line profile of an AFM image of that specific channel). Fig. $3.9 \mathrm{~b}$ ) shows a force-distance curve measured between a SSS Si AFM tip and $\mathrm{SrTiO}_{3}(001)$ surface at RT and $\mathrm{PO}_{2}=10^{-1}$ mbar $\mathrm{O}_{2}$. The measured force-distance curve shows a qualitative agreement with force-distance curves reported in previous work [33].

It should be noted that the cantilever temperature shows a strong dependence on its distance from the heated sample surface. Therefore, a shift in $f_{0}$ is observed during the tip-sample approach. Once the cantilever amplitude is below the amplitude setpoint during tip-sample approach, it is assumed that the AFM tip is in close vicinity of the substrate surface. From the SPM electronics channel read-out, it was deduced that the SSS cantilever oscillation amplitude $A_{\text {osc }}$ decreases in a time frame of $\approx 0.1 \mathrm{~s}$, when the tip comes in close vicinity of the sample surface at RT. The same experiment was repeated at $T=600{ }^{\circ} \mathrm{C}$ and $P O_{2}=10^{-1} \mathrm{mbar}$ $\mathrm{O}_{2}$ revealing that the $A_{\mathrm{osc}}$ is decreasing in a time frame of $\approx 10$ seconds before it is in close vicinity of the sample surface. In the latter case, $-\frac{d A_{\mathrm{osc}}}{d d_{\mathrm{tip} \rightarrow \mathrm{sur}}}$ increases gradually when the distance $d_{\mathrm{tip} \rightarrow \text { sur }}$ between AFM tip and sample surface becomes smaller. Here, the coarse approach stopped without having the AFM tip positioned on the $\mathrm{SrTiO}_{3}(001)$, since $A_{\text {osc }}$ crosses the setpoint $A_{\text {set }}$.

During thermal stabilization, a negative $f_{0}$ drift has been observed for a Si cantilever. Fig. 3.10 a) shows results of an experiment, where a $\operatorname{SrTiO}_{3}(001)$ substrate was heated from RT up to $T=600{ }^{\circ} \mathrm{C}$ at $P O_{2}=10^{-1}$ mbar $\mathrm{O}_{2}$ and at an AFM tip - substrate distance $=19 \mu \mathrm{m}$. Thereafter, the AFM tip was brought closer to the $\mathrm{SrTiO}_{3}(001)$ surface in $5 \mu \mathrm{m}$ steps and an amplitude vs. frequency curve was measured waiting for each step $30 \mathrm{~min}$. This was repeated up to the AFM tip was in close vicinity of the surface. In this experiment, an SSS Si cantilever $f_{0}=156.865 \mathrm{kHz}$ containing a $\mathrm{FWHM}=22 \mathrm{~Hz}$ was used.

In Fig. $3.10 \mathrm{a}$ ), the drift in $f_{0}$ is larger in case the AFM tip is closest to the heated surface. In addition, it can be seen that $f_{0}$ drift is larger than FWHM of the cantilever extracted from amplitude vs. frequency curve. Therefore, the cantilever amplitude gradually decreases during the coarse approach when kept the same selected $f_{0}$. To solve this issue, AR5 Si cantilevers are used as their $\mathrm{FWHM} \approx 200 \mathrm{~Hz}$ is an order of magnitude larger, see Fig. $3.10 \mathrm{~b}$ ). It turned out that a cantilever with a FWHM $=201 \mathrm{~Hz}$ could be successfully used to approach 

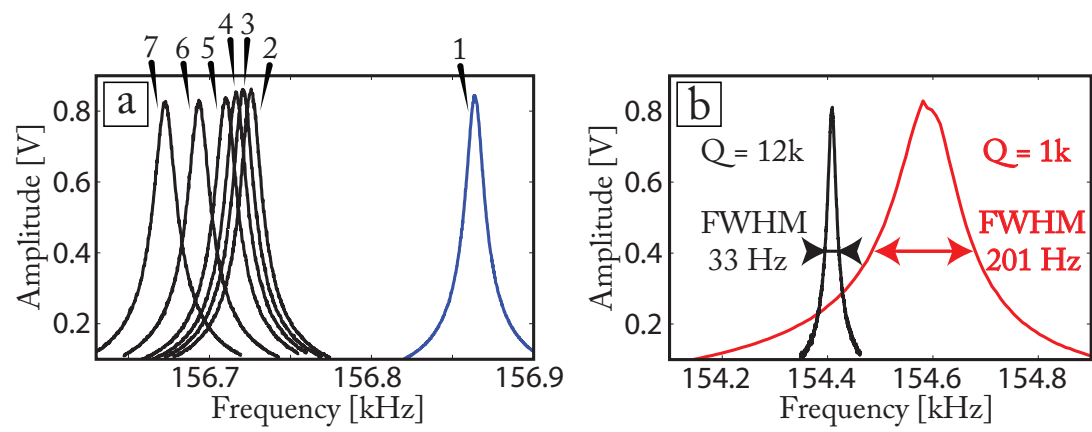

Fig. 3.10 Amplitude vs. frequency cantilever curves at $\mathrm{PO}_{2}=10^{-1} \mathrm{mbar}_{2}$, a) curves at different distances between AFM tip and $\mathrm{SrTiO}_{3}(001)$ surface $d_{\text {tip } \rightarrow \text { sur }}(1) \mathrm{RT},(2)$ after $2 \mathrm{~h}$ thermal stabilization at $600{ }^{\circ} \mathrm{C}$ at $d_{\text {tip } \rightarrow \text { sur }}=19 \mu \mathrm{m},(3)$ after $30 \mathrm{~min}$ at $600{ }^{\circ} \mathrm{C}$ at $d_{\mathrm{tip} \rightarrow \text { sur }}=14 \mu \mathrm{m},(4)$ after $30 \mathrm{~min}$ at $600{ }^{\circ} \mathrm{C}$ at $d_{\mathrm{tip} \rightarrow \text { sur }}=9 \mu \mathrm{m},(5)$ after $30 \mathrm{~min}$ at $600{ }^{\circ} \mathrm{C}$ at $d_{\text {tip } \rightarrow \text { sur }}=4 \mu \mathrm{m},(6)$ after $30 \mathrm{~min}$ at $600{ }^{\circ} \mathrm{C}$ at $d_{\text {tip } \rightarrow \text { sur }}=1 \mu \mathrm{m}$ and (7) after $30 \mathrm{~min}$ at $600{ }^{\circ} \mathrm{C}$ AFM tip on surface. Frequency shift $\Delta f \approx 200 \mathrm{~Hz}$ of the cantilever during warming up of the $\mathrm{SrTiO}_{3}(001)$ sample (1) from RT to (2) $600{ }^{\circ} \mathrm{C}$ and waiting for 30 min after approaching AFM tip to sample surface, b) curves of SSS $\left(Q_{\mathrm{c}}=12 \mathrm{k}\right)$ and $\operatorname{AR} 5\left(Q_{\mathrm{c}}=1 \mathrm{k}\right)$ Si cantilever at RT. The FWHM of the cantilever is an important parameter for the AFM operation stability and coarse approach.

the AFM tip in close vicinity of the sample surface and stable AFM imaging could be performed at $T=600{ }^{\circ} \mathrm{C}$ and $P O_{2}=10^{-1} \mathrm{mbar}_{2}$.

\subsubsection{AFM imaging}

Here, the AFM imaging performance in air, under PLD conditions and while growing a PLD thin film is demonstrated and discussed.

\section{AFM scan speed under air and PLD conditions}

First, TM-AFM measurements are performed to test the AFM scan speed performance in air $^{15}$, see Fig. 3.11. The scan speed is increased going the Fig. 3.11 from left to right illustrating the AFM performance by imaging $\mathrm{SrTiO}_{3}(001)$ substrate steps of $0.4 \mathrm{~nm}$. At conventional AFM scan speeds, with a $2 \mathrm{~Hz}$ line rate having an acquisition time of $256 \mathrm{~s} /$ frame, the subnanometer $\mathrm{SrTiO}_{3}$ substrate steps are clearly resolved. The $0.4 \mathrm{~nm} \mathrm{SrTiO}_{3}(001)$ steps are visible in the AFM height profile. From this profile, a peak-to-peak z-noise was found $<0.1 \mathrm{~nm}$ at a

15 The vibrational level is lower under vacuum conditions than in air as active damping control units only can be used under vacuum conditions. 


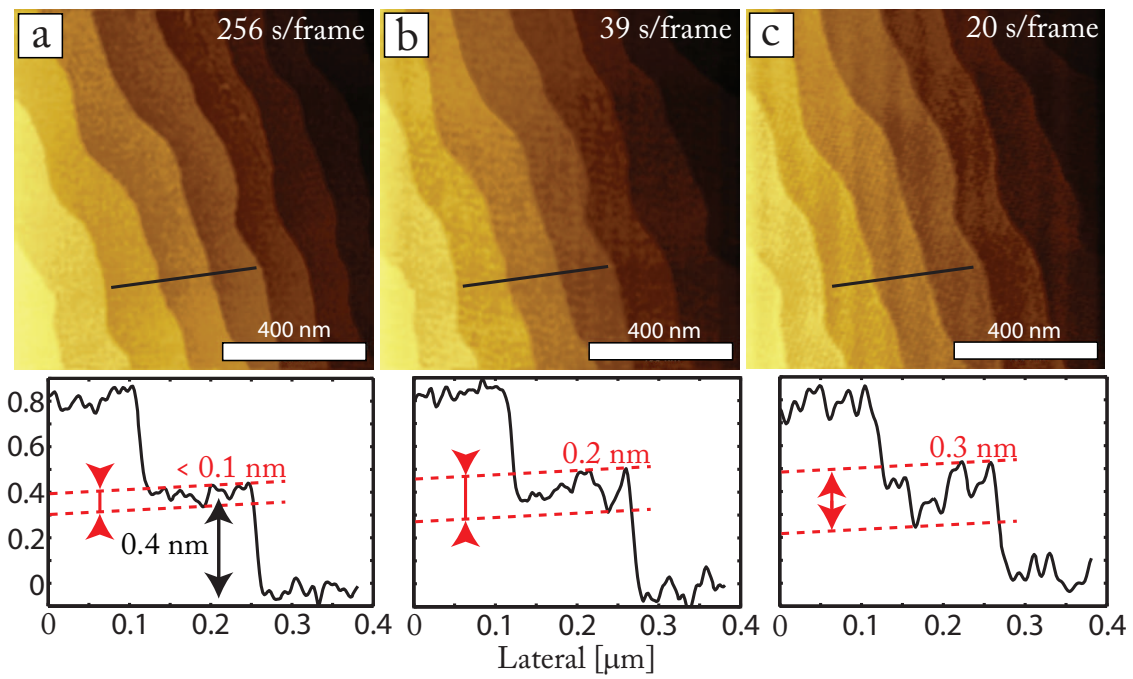

Fig. 3.11 TM-AFM $1 \times 1 \mu \mathrm{m}^{2} 512 \times 512$ pixels ${ }^{2}$ images of a $\mathrm{SrTiO}_{3}(001)$ substrate in air at RT with in the bottom panels the corresponding height profiles. The scan speed is increased from left right from $256 \mathrm{~s} /$ frame to $20 \mathrm{~s} /$ frame. The resolution decreases significantly at a scan speed of $20 \mathrm{~s} /$ frame due to the limitation of the cantilever resonance frequency $f_{0}$. The AFM flexure scanner is not the limiting factor in terms of speed. A resonance frequency of $1.6 \mathrm{kHz}$ appears caused by the mounting bracket connection with the coarse approach stage.

bandwidth of $2.0 \mathrm{kHz}$, see Fig. $3.11 \mathrm{a}$ ). The AFM resolution slightly decreases as the acquisition time is reduced to $39 \mathrm{~s} /$ frame (bandwidth $13.4 \mathrm{kHz}$ ) due to a pixel dwell time decrease. An increase in peak-to-peak z-noise has been observed for 39 $\mathrm{s} /$ frame compared to $256 \mathrm{~s} /$ frame. Furthermore, a smaller AFM acquisition time per frame of $20 \mathrm{~s} /$ frame (bandwidth $=26.2 \mathrm{kHz}$ ) results in a even larger increase of peak-to-peak z-noise. At this AFM image acquisition time, a peak-to-peak z-noise $\approx 0.3 \mathrm{~nm}$ has been measured. In addition, a resonance frequency of $1.6 \mathrm{kHz}$ is appearing, a vibrational mode of the mounting bracket connection with the coarse approach stage.

The increase in peak-to-peak z-noise can be attributed to two sources; (1) the electronic noise increases proportional to $\sqrt{f_{\mathrm{bw}}}$, whereas the LPM electronics has been proven as a suitable SPM electronics system for resolving surfaces with atomic resolution at acquisition rates up to 80 frames/s $(128 \times 128$ pixels, maximum tip velocity $=0.3 \mathrm{~mm} / \mathrm{s}$ ) on the one hand $[35,185,186]$. On the other hand, the SIS electronics bandwidth is limited to $400 \mathrm{kHz}$ due to its photodetector. Therefore, only cantilevers with a $f_{0}<400 \mathrm{kHz}$ are applicable. A reduced pixel dwell time results in less oscillations used to determine a RMS value for every single pixel. 
To reduce the AFM z-noise at low acquisition times per frame, a high bandwidth detection system needs to be developed with a peak-to-peak z-noise $<0.1 \mathrm{~nm}$ such that $\mathrm{MHz}$ frequency cantilevers can be used. In addition, (2) a mechanical resonance appearing from the mounting bracket / coarse approach connection increases the peak-to-peak noise in Fig. $3.11 \mathrm{c}$ ). A re-design of the mounting bracket with focus on more symmetric positions of its screw holes can reduce the vibrational strength and therefore extending the AFM scan speed without exceeding a peak-to-peak z-noise of $0.1 \mathrm{~nm}$.

Secondly, the AFM scan speed has been tested at $P_{2}=10^{-1} \mathrm{mbar}_{2}$. Step resolution is hardly visible after a decrease in acquisition time from $256 \mathrm{~s} /$ frame to $39 \mathrm{~s} /$ frame under these conditions. A significant increase of cantilever quality factor $Q_{\mathrm{c}}$ from several hundred in air to tens of thousand at $P O_{2}=10^{-1} \mathrm{mbar}$ $\mathrm{O}_{2}$ has been reported [33]. The transient time $\tau_{\mathrm{cTM}}$ is proportional to $Q_{\mathrm{c}}$ and therefore decreases the cantilever bandwidth $\frac{f_{0}}{Q_{c}}$. The maximum achievable AFM scan speed can be extended by both (1) using high frequency cantilevers in the order of $\mathrm{MHz}$ and (2) increase of detector bandwidth (in this system especially the bandwidth of the photodetector without noise increase). Furthermore, FMAFM is more favorable to extend the AFM scan speed and is typically applied under vacuum conditions. In FM-AFM mode, the transient time $\tau_{\text {cFM }}$ is nearly independent of $Q_{\mathrm{c}}$, but then the bandwidth of PLL demodulator is limiting the AFM scan speed. As mentioned in Ch. 2, a lot of progress has been made recently to extend the demodulator bandwidth enabling real-time FM-AFM [65, 182].

\section{AFM imaging under PLD conditions}

In situ AFM images shown in Fig. 3.12 are recorded under typical PLD conditions. In Fig. 3.12 a), an FM-AFM image is depicted of a $\mathrm{SrTiO}_{3}(001)$ substrate containing unit cell vacancy islands of $0.4 \mathrm{~nm}$ deep recorded in a background pressure of $10^{-6}$ mbar at RT [19]. In this FM-AFM image, the vertical resolution is similar to the $\mathrm{z}$-noise level $(<0.1 \mathrm{~nm})$ and unit cell $(0.4 \mathrm{~nm})$ deep vacancy islands are obtained. Lateral vacancy island sizes of $\approx 20 \mathrm{~nm}$ are visible and measured. In the lateral direction, the AFM resolution was determined to be $\approx 10 \mathrm{~nm}$ similar to the tip apex. Lateral AFM resolution is determined by measuring $\mathrm{SrTiO}_{3}(001)$ step-edge broadening [186]. In this pressure regime, the FM-AFM imaging mode is typically used due to the high $Q_{\mathrm{c}}$ for image stability reasons and lower transient time compared to TM-AFM $[157,178]$. In this work, TM-AFM imaging on a $\mathrm{SrTiO}_{3}(001)$ substrate at $10^{-6}$ mbar and RT has a similar lateral AFM resolution 


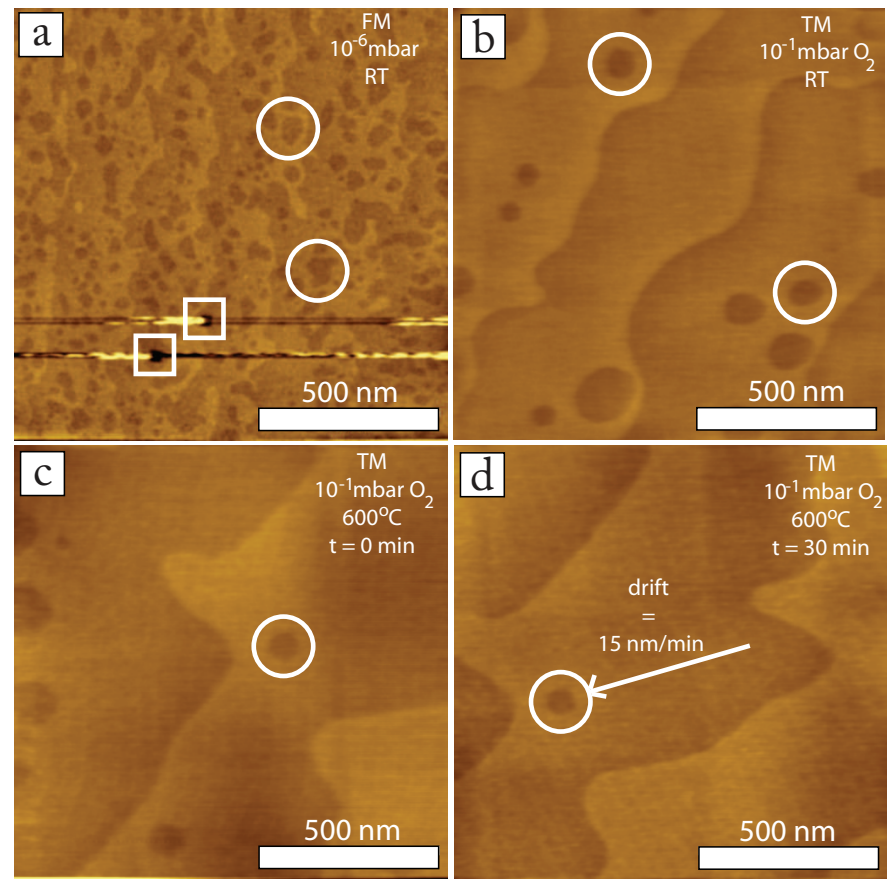

Fig. 3.12 In situ AFM $1.2 \times 1.2 \mu \mathrm{m}^{2}\left(512 * 512\right.$ pixels $\left.{ }^{2}\right)$ images of $\mathrm{SrTiO}_{3}(001)$ substrates under different conditions (and different miscut angles) recorded with an acquisition time of $256 \mathrm{~s} /$ frame. a) FM-AFM image, $\Delta f=-28 \mathrm{~Hz}, A_{\text {osc }}=10 \mathrm{~nm}$, at RT and $P=10^{-6}$ mbar, b) TM-AFM image, $A_{\text {osc }}=44 \mathrm{~nm}$, at RT and $P O_{2}=10^{-1}$ mbar, c) TM-AFM image, $A_{\mathrm{osc}}=44 \mathrm{~nm}$, at $\mathrm{T}=600{ }^{\circ} \mathrm{C}$ and $P O_{2}=10^{-1} \mathrm{mbar} \mathrm{O}_{2}$, d) TM-AFM image, $A_{\mathrm{osc}}=44 \mathrm{~nm}$, at $\mathrm{T}=600{ }^{\circ} \mathrm{C}$ and $P O_{2}=10^{-1}$ mbar $\mathrm{O}_{2}$ after $30 \mathrm{~min} \mathrm{AFM}$ imaging. Circle markers surround unit cell vacancy islands with a depth of $0.4 \mathrm{~nm}$ and square markers surround etch pits of several unit cells deep.

and operating stability compared to FM-AFM ${ }^{16}$.

In Fig. 3.12 b), a TM-AFM image is depicted of a $\mathrm{SrTiO}_{3}(001)$ substrate at $\mathrm{PO}_{2}=10^{-1}$ mbar $\mathrm{O}_{2}$, which is a typical PLD pressure to deposit perovskite oxide films ensuring good crystal quality, properties, stoichiometric transfer and $2 \mathrm{D}$ growth [126]. At $\mathrm{PO}_{2}=10^{-1}$ mbar $\mathrm{O}_{2}$, the free cantilever amplitude $A_{\mathrm{osc}}$ was set to $120 \mathrm{~nm}$ and is the most critical parameter for AFM imaging. The lateral AFM resolution on terraces is lowered compared to $10^{-6}$ mbar $\mathrm{O}_{2}$ as a $\mathrm{SrTiO}_{3}(001)$ step broadening is $\approx 20 \mathrm{~nm}$ at $P O_{2}=10^{-1} \mathrm{mbar}_{2}$. A smallest lateral vacancy island size of $\approx 34 \mathrm{~nm}$ was found in the AFM image in Fig. 3.12 b). An average in-plane drift of $2 \mathrm{~nm} / \mathrm{min}$ is measured over a period of $3.5 \mathrm{~h}$

${ }^{16}$ In FM-AFM and TM-AFM measurements at $10^{-6}$ mbar and a sample temperature $T=$ RT, SSS Si cantilevers of $190 \mathrm{kHz}$ were used with a tip apex of $\approx 10 \mathrm{~nm}$. 
under these conditions.

The same AFM settings have been used at $T=600{ }^{\circ} \mathrm{C}$ compared to RT. Due to thermal drift, a lower $f_{0}$ has been found, where $\Delta f$ is typically $-200 \mathrm{~Hz}$. Vacancy islands are still obtained at $P O_{2}=10^{-1}$ mbar $\mathrm{O}_{2}$ and $T=600{ }^{\circ} \mathrm{C}$. The AFM noise increase due to temperature is negligible based on the fact that $\mathrm{z}$-noise level and $\mathrm{SrTiO}_{3}(001)$ step broadening remained at $T=600{ }^{\circ} \mathrm{C}$ [33]. In Fig. $3.12 \mathrm{c}$ ), a minimum vacancy island of $40 \mathrm{~nm}$ in diameter was found. It was noticed that AR5 Si cantilevers operate more stable at $T=600{ }^{\circ} \mathrm{C}$ compared to SSS cantilevers due to an order of magnitude larger FWHM in amplitude vs. frequency curve, see Fig 3.10. Using only a power of 5 Watt, the sample temperature is $T=400{ }^{\circ} \mathrm{C}$ in air and $T=600{ }^{\circ} \mathrm{C}$ at $P O_{2}=10^{-1}$ mbar $\mathrm{O}_{2}$. Under these conditions, a drift of $9 \mathrm{~nm} / \mathrm{min}$ and $15 \mathrm{~nm} / \mathrm{min}$ is measured in-plane, respectively. After hours of stable AFM imaging under oxide PLD conditions, the z-piezo stayed within range $( \pm 700 \mathrm{~nm})$.

\section{AFM during and after PLD}

$\mathrm{BiFeO}_{3}$ has been deposited as prototypical $\mathrm{ABO}_{3}$ film using the described PLD system. This material has multiferroic properties and its growth and property relation is intensively studied [191, 202, 203, 204, 205]. It is reported that $\mathrm{BiFeO}_{3}$ tends to grow in several type of domains $\left(71^{\circ}\right.$ and $\left.109^{\circ}\right)$ by modifying the $\mathrm{SrTiO}_{3}(001)$ termination [191]. Dependent on the substrate termination, $\mathrm{BiFeO}_{3}$ grows either $1 \mathrm{D} / 2 \mathrm{D}$ or $3 \mathrm{D}$. Here, $\mathrm{BiFeO}_{3}$ films have been grown on $\mathrm{TiO}_{2}$ terminated $\mathrm{SrTiO}_{3}(001)$ substrates expecting $3 \mathrm{D}$ growth. $\mathrm{BiFeO}_{3}$ was deposited with a laser fluence of $2.0 \mathrm{~J} / \mathrm{cm}^{2}, \mathrm{PO}_{2}=0.3$ mbar $\mathrm{O}_{2}, \mathrm{~T}=600-670{ }^{\circ} \mathrm{C}$, repetition rate $f_{\text {rep }}=0.5 \mathrm{~Hz}$ all settings similar to previous work where only a target-substrate distance $^{17}$ of $45 \mathrm{~mm}$ is used instead of $55 \mathrm{~mm}$ [191].

Fig. 3.13 shows AFM images obtained after and during $\mathrm{BiFeO}_{3}$ deposition. In Fig. $3.13 \mathrm{a}$ ), an ex situ $\mathrm{AFM}$ image ${ }^{18}$ is depicted of $\mathrm{BiFeO}_{3}$ film after deposition of 1000 pulses at $670{ }^{\circ} \mathrm{C}^{19}$. This $\mathrm{BiFeO}_{3}$ film was deposited as a reference to reported literature [191]. It is reported that $\mathrm{BiFeO}_{3}$ grows $3 \mathrm{D}$ on $\mathrm{TiO}_{2}$ terminated $\mathrm{SrTiO}_{3}(001)$, which is similar to the results obtained here. Terraces with on top 3D islands are visible after $1000 \mathrm{PLD}$ pulses of $\mathrm{BiFeO}_{3}$ deposited on $\mathrm{TiO}_{2}$ terminated $\mathrm{SrTiO}_{3}(001)$. The $3 \mathrm{D} \mathrm{BiFeO}_{3}$ islands on top of the terraces suggest that next layer nucleation starts before a previous layer is completely covered. Island step

\footnotetext{
17 Target-substrate distance was here limited to $45 \mathrm{~mm}$ due to a irregular thickness of the rod on the $\mathrm{BiFeO}_{3}$ target holder.

18 After $\mathrm{BiFeO}_{3}$ deposition, ex situ AFM images were taken with a Bruker Dimension Icon.

19 The thickness of the $\mathrm{BiFeO}_{3}$ film $d_{\text {film }}$ is expected to be $d_{\text {film }} \approx 3-4 \mathrm{~nm}$.
} 

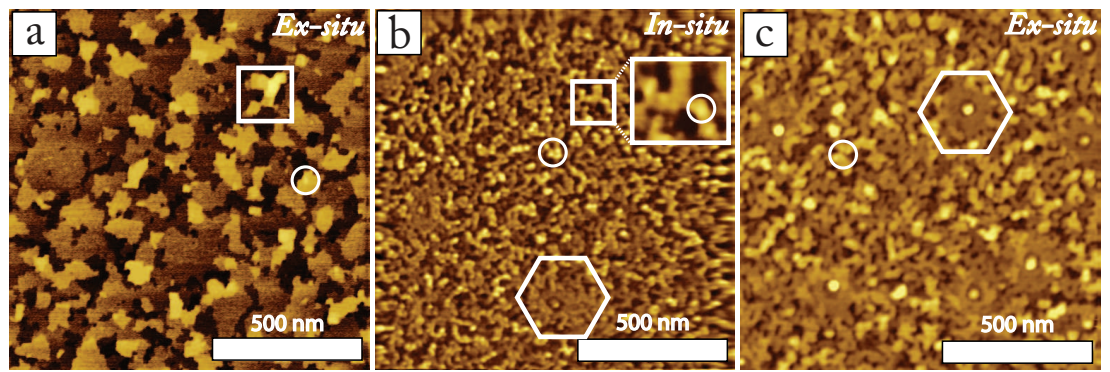

Fig. $\quad 3.13 \quad$ TM-AFM $\quad 1.2 \times 1.2 \quad \mu^{2} \quad\left(512 \times 512 \quad\right.$ pixels $\left.{ }^{2}\right)$ images, acquisition time $=256 \mathrm{~s} /$ frame, after 1000 pulses of $\mathrm{BiFeO}_{3}$ deposited on $\mathrm{SrTiO}_{3}(001)$ a) ex situ at RT and in air after deposition at $T=670{ }^{\circ} \mathrm{C}$, b) in situ at $T=600{ }^{\circ} \mathrm{C}$ and $P_{2}=0.3$ mbar $\mathrm{O}_{2}$ after 1000 pulses of $\mathrm{BiFeO}_{3}$ deposited on $\mathrm{SrTiO}_{3}(001)$, c) ex situ at RT and in air after deposition at $T=600{ }^{\circ} \mathrm{C}$. Square markers point to $3 \mathrm{D} \mathrm{BiFeO}_{3}$ islands, circle markers point to small $\mathrm{BiFeO}_{3}$ islands and polygon markers point to larger islands.

heights are found of $0.2 \mathrm{~nm}, 0.4 \mathrm{~nm}$ and its multiples up to a maximum peak-topeak height of $3 \mathrm{~nm}$. Islands of $25-30 \mathrm{~nm}$ in lateral size are obtained on top of random shaped islands. From these results, it is expected that $\mathrm{BiFeO}_{3}$ growth will continue 3D, similar to what has been reported [191].

In Fig. $3.13 \mathrm{~b}$ ), an in situ AFM image is presented, which was recorded at $T=600{ }^{\circ} \mathrm{C}$ and $\mathrm{PO}_{2}=0.3$ mbar $\mathrm{O}_{2}$ after 1000 pulses of $\mathrm{BiFeO}_{3}$. The AFM image was taken after $\mathrm{BiFeO}_{3}$ deposition, sample transfer from PLD to AFM and an AFM stabilization time of $2 \mathrm{~h}$ at the AFM position. After thermal stabilization, AFM was started with similar settings used for imaging of a $\operatorname{SrTiO}_{3}(001)$ substrate. It became clear that due to an increase in surface roughness after deposition, the integral gain of AFM electronic feedback had to be increased strongly to visualize the smallest $\mathrm{BiFeO}_{3}$ islands. Stable AFM imaging was continued for several hours on $\mathrm{BiFeO}_{3}$ without thermally drifting out of the Z-range. Thermal drift causes small distortions at AFM image edges. Smallest lateral $\mathrm{BiFeO}_{3}$ island sizes of 20 - $30 \mathrm{~nm}$ have been measured, similar to the results obtained with an $e x$ situ $\mathrm{AFM}$ on $\mathrm{BiFeO}_{3}$ after deposition at $T=670{ }^{\circ} \mathrm{C}$ [191]. Some of these small islands are surrounded by a white circle marker in Fig. 3.13. The same lateral island sizes have been found after a zoom-in from $1.2 \times 1.2 \mu^{2}$ to $0.8 \times 0.8 \mathrm{\mu m}^{2}$. In Fig. $3.13 \mathrm{~b}$ ), the square white marker representing a zoom-in of a $\mathrm{BiFeO}_{3}$ taken from an $0.8 \times 0.8 \mathrm{\mu m}^{2}$ AFM image.

Afterwards, the $\mathrm{BiFeO}_{3}$ film grown on $\mathrm{SrTiO}_{3}(001)$ at $T=600{ }^{\circ} \mathrm{C}$ had a cooldown of $\approx 15^{\circ} \mathrm{C} / \mathrm{min}$ in its deposition pressure of $\mathrm{PO}_{2}=0.3 \mathrm{mbar} \mathrm{O}_{2}$. Once the sample 
reached $\mathrm{RT}$, it was exposed to an maximum $\mathrm{O}_{2}$ flow up to atmospheric pressure. In Fig. $3.13 \mathrm{c}$ ), an ex-situ AFM image is depicted of $\mathrm{BiFeO}_{3}$ after $\mathrm{O}_{2}$ exposure up to atmospheric pressure. The AFM image in Fig. $3.13 \mathrm{c}$ ) is slightly different compared to Fig. $3.13 \mathrm{~b}$ ). One difference is that $\mathrm{BiFeO}_{3}$ islands of $\approx 20-30$ $\mathrm{nm}$ are hardly visible in ex situ AFM image. Both AFM images have in common that $\mathrm{BiFeO}_{3}$ islands within polygon markers are similar in size. Most likely, the AFM tip apex could be different as a Bruker $320 \mathrm{kHz}$ cantilever has been used during ex situ AFM. Moreover, $Q_{\mathrm{c}}$ decreases more than an order of magnitude from $\mathrm{PO}_{2}=10^{-1}$ mbar $\mathrm{O}_{2}$ up to atmospheric pressure.

This study reveals that $\mathrm{BiFeO}_{3}$ can be imaged using a Si AFM tip at $T=600{ }^{\circ} \mathrm{C}$ and $\mathrm{PO}_{2}=0.3$ mbar $\mathrm{O}_{2}$ after deposition on a $\mathrm{TiO}_{2}$ terminated $\mathrm{SrTiO}_{3}(001)$ substrate. Neck formation has not been observed using this tip-sample combination under these conditions. One of the ideas is to deposit $\mathrm{BiFeO}_{3}$ on mixed terminated $\mathrm{SrTiO}_{3}(001)$ in order to study growth (front evolution) differences on both $\mathrm{SrO}$ and $\mathrm{TiO}_{2}$ termination. However, AFM imaging (Si tip) on mixed terminated $\mathrm{SrTiO}_{3}$ substrates results in neck formation at $T=600{ }^{\circ} \mathrm{C}$ and $\mathrm{PO}_{2}=0.1$ mbar $\mathrm{O}_{2}$, while stable AFM imaging was achieved on a $\mathrm{TiO}_{2}$ deposited film on $\mathrm{TiO}_{2}$ terminated $\mathrm{SrTiO}_{3}(001)$ under the mentioned conditions. From the described measurements and the neck formation of a $\mathrm{Si}$ AFM tip with a $\mathrm{SrRuO}_{3}$ film ( $\mathrm{SrO}$ termination) at $T=600{ }^{\circ} \mathrm{C}$ and $\mathrm{PO}_{2}=0.1$ mbar $\mathrm{O}_{2}$, it seems plausible to argue that a Si AFM tip forms a neck if the surface contains a SrO top layer [8, 33]. For this tip-sample combination, an other tip material/tip coating needs to be selected.

\subsection{Conclusions}

The designed AFM integrated in a PLD system enables in situ oxide PLD growth studies on a microscopic scale. The following specifications have been fulfilled:

- The AFM operates at sample temperatures from RT up to $700{ }^{\circ} \mathrm{C}$ and at pressures ranging from $10^{-6}$ to 1 mbar $\mathrm{O}_{2}$.

- Subnanometer resolution is demonstrated on a $\mathrm{SrTiO}_{3}(001)$ substrate and on a $\mathrm{BiFeO}_{3}$ film deposited on $\mathrm{SrTiO}_{3}(001)$ at $\mathrm{T}=600{ }^{\circ} \mathrm{C}$ and $\mathrm{PO}_{2}=10^{-1} /$ $\mathrm{PO}_{2}=0.3$ mbar $\mathrm{O}_{2}$, respectively. A bare Si AFM tip was used in this experiment and no neck formation was obtained.

- A similar XYZ vibrational level has been measured under PLD conditions compared to a standard commercial available AFM having a $<0.1 \mathrm{~nm}$ height resolution. 
- AFM imaging on a PLD grown $\mathrm{BiFeO}_{3}$ film revealed that $\mathrm{BiFeO}_{3}$ islands can be measured at least down to lateral sizes of $20 \mathrm{~nm}$ at $T=600{ }^{\circ} \mathrm{C}$ and $\mathrm{PO}_{2}=0.3$ mbar $\mathrm{O}_{2}$.

- A thermal in-plane drift has been found of $15 \mathrm{~nm} / \mathrm{min}$ during AFM imaging at $P_{2}=10^{-1}$ mbar $\mathrm{O}_{2}$ and $T=600^{\circ} \mathrm{C}$.

- Stable AFM imaging is performed under metal-oxide PLD conditions for a few hours without exceeding the Z-piezo range of $\pm 700 \mathrm{~nm}$.

However, the maximum achievable line rate of the designed AFM is limited to few $\mathrm{Hz}$ under PLD conditions without exceeding a peak-to-peak z-noise of $0.1 \mathrm{~nm}$. In order to extend the AFM scan speed to monitor oxide growth kinetic processes under PLD conditions, the cantilever and detection bandwidth have to be increased to the order of $\mathrm{MHz}$ or FM-AFM needs to be applied with a PLL demodulator bandwidth in the order of several hundreds of $\mathrm{kHz}$. 


\title{
Chapter 4
}

\section{Fast side approach for atomic force microscopy}

\begin{abstract}
The commonly used tip-sample approach in atomic force microscopy (AFM) is a timeconsuming procedure resulting in low sample throughput needed for studying the growth front during pulsed laser deposition (PLD). Therefore, monitoring the nucleation density and growth of islands within subseconds after a deposition pulse requires a different tip-sample approach, decreasing the approach time. In this chapter, a fast tip-sample approach method is proposed in order to image a specific sample surface location after back and forth sample transfer between monitoring and deposition. This approach enables "quasi" real-time AFM monitoring of the growth kinetics during PLD thin film growth.
\end{abstract}

W.A. Wessels, J.J. Broekmaat, R.J.L. Beerends, G. Koster and G. Rijnders. Fast and gentle side approach for atomic force microscopy, Review of Scientific Instruments 84, 123704 (2013). 


\subsection{Introduction}

The latest developments in atomic force microscopy (AFM) [30] are aimed at decrease in acquisition time, which is defined here as the time to measure the surface topography with $256 \times 256$ pixels per frame [38], and to operate in dynamic mode instead of contact mode. The dynamic operating mode is considered non-destructive as compared to the contact operating mode and is therefore often the preferred imaging method. Currently, the time to capture a topographical image is reduced from minutes towards (sub)seconds in contact as well as dynamic operating modes [36, 37, 39, 61]. These developments have resulted in the first commercial instruments capturing images at a rate of several frames per second $[35]^{1}$. The realization of high-speed AFM's (HS-AFM) is an important step in enabling real-time monitoring of surface dynamics, such as growth kinetic processes. For example, real-time AFM monitoring would increase the understanding of oxide thin film growth during pulsed laser deposition (PLD).

AFM and PLD are preferably positioned at a geometrically different position to prevent significant tip degradation and hindering of deposition on the as-grown surface. The sample surface can be monitored after e.g (1) the temporarily AFM scanner transfer towards the sample surface between deposition pulses or (2) using a lateral sample transfer back and forth between AFM and PLD [33]. In this work, the back and forth sample transfer has been chosen based on lower inertia. Using a sample transfer mechanism, surface monitoring can be continued only after deposition pulses if (1) the sample is repositioned at the AFM position and (2) the AFM tip has approached the sample surface. Both procedures should be fast in order to monitor growth kinetics with characteric relaxation times of (sub)seconds [17, 18]. Regarding the sample transfer, some vacuum compatible motors $^{2}$ enable sample transfer times in the order of subseconds (over $\left.60 \mathrm{~mm}\right)^{3}$. However, the commonly used tip-sample approach is still the bottleneck as this tedious procedure takes (sub)minutes.

Among tip-sample approach methods, the proposed side approach is one of the few methods showing a significant decrease in tip-sample approach time [33, 206]. Its principle differs from the commonly used tip-sample approach as the sample is transferred lateral towards the AFM tip instead of e.g. perpendicular to the tip. The strength of the side approach can be attributed to the fact that the tip-sample

\footnotetext{
${ }^{1}$ Bruker Dimension Fastscan and Cypher S AFM.

${ }^{2}$ PI ultrasonic piezo motors and Nanomotion motors drive stages with a speed of $\approx 250 \mathrm{~mm} / \mathrm{s}$. These motors exhibit reposition repeatability errors of around hundred $\mathrm{nm}$.

${ }^{3}$ Vacuum compatible linear electro motors can drive stages even up to speeds of several $\mathrm{m} / \mathrm{s}$. The implementation has been considered. However, it uses dynamic instead of static repositioning.
} 
approach is part of the sample transfer. The side approach is suited as approach mechanism especially when (1) AFM and PLD are laterally in position separated and (2) the back and forth sample transfer is used.

Previously, a side approach time of $\approx 4 \mathrm{~s}$ is measured having a stage reposition repeatability error of $\pm 2 \mu \mathrm{m}$, where a belt drive was used as stage drive mechanism [33]. However, here the (side) approach is in the order of seconds, where the typical timescale of growth kinetic processes of oxides during PLD are in the order of (sub)seconds $[17,18]$. Another drawback, the reported reposition repeatability error is in the order of the typical size of the area of interest. This makes it nearly impossible to monitor local nucleation and growth phenomena at the same surface location.

The aim of this chapter is to demonstrate and test the performance of the side approach using a faster sample transfer and a better reposition repeatability as the side approach is one of the crucial steps in enabling real-time monitoring of the growth kinetics. First, the specifications and requirements of a tip-sample approach are described. Thereafter, the concept of the side approach is explained. A design to validate the performance is presented in the following section. After the design section, the performance of the side approach using a faster sample transfer is shown. The chapter ends by presenting the conclusions.

\subsection{Specifications needed for AFM monitoring of PLD growth}

Many oxide growth kinetic processes during PLD occur at timescales of (sub)seconds, confirmed by reflection high-energy electron diffraction (RHEED) measurements [17, 18]. The RHEED specular intensity is a measure of the surface step density, which can vary with coverage, nucleation density/radius and supersaturation. Besides that, the relaxation behavior of the RHEED intensity depends on the adatom density as well ${ }^{4}$. Therefore, the single pulse relaxation is characteristic for both the step density evolution and the adatom density decay after a deposition pulse shown in Fig. 4.1 [140]. The adatom density decays after a deposition pulse as adatoms diffuse and become attached to island edges. At coverages close to unity (coverage $\approx 0.7-1.0$ ), relaxation times are measured assuming most of the deposited material is deposited on top of the islands. The evolution of the step density is here neglected and only the timeconstant of diffusing adatoms is considered [18]. Relaxation times of $\tau=0.2-6.2 \mathrm{~s}$ are measured for homoepitaxial $\mathrm{SrTiO}_{3}$ growth,

\footnotetext{
${ }^{4}$ Diffusive adatoms act as diffusive scatterers for electrons.
} 


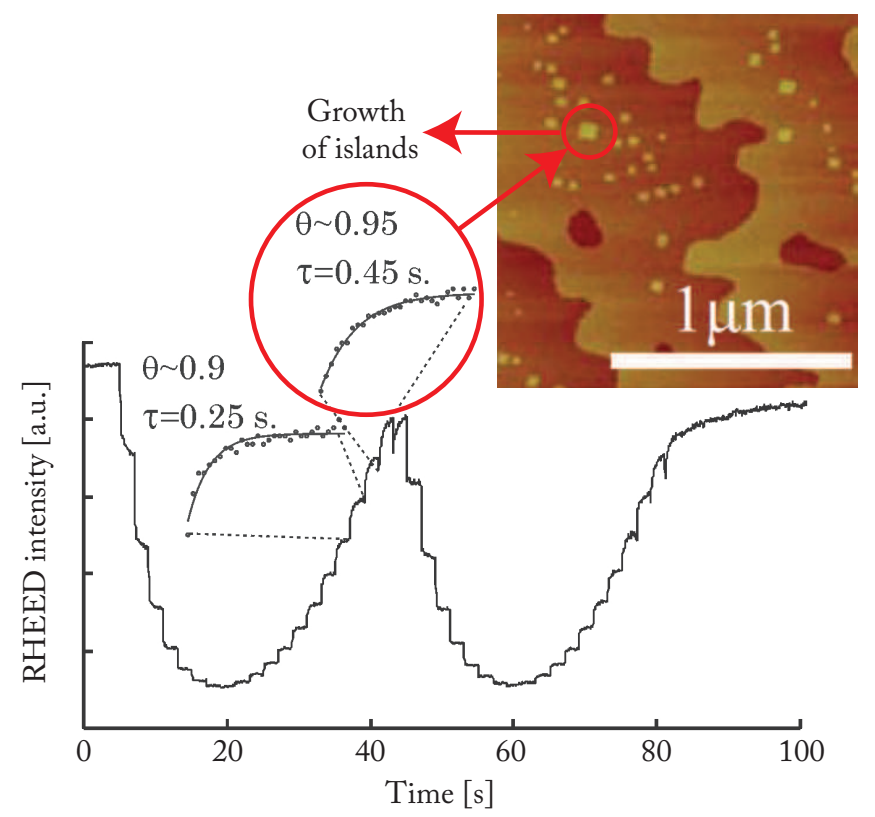

Fig. 4.1 RHEED growth monitoring during homoepitaxial $\mathrm{SrTiO}_{3} \mathrm{PLD}_{\text {growth. A }}$ complete $\mathrm{SrTiO}_{3}$ monolayer is grown every single RHEED oscillation (here $\approx 40 \mathrm{~s}$ ). The relaxation behavior of the RHEED intensity varies with both the evolution of the step density and the decay of the adatom density. The relaxation time $\tau$ can be extracted of a single deposition pulse, where $\tau$ is the timescale of e.g. the adatom density decay during oxide PLD $[17,18]$. The adatom density decays as adatoms diffuse and become attached to island edges resulting in lateral growth of islands. The growth front needs to be monitored in timescale $\tau$ using AFM in order to measure to (lateral) island growth. Figs. included with permission from Koster and Broekmaat $[17,33]$.

where $\tau$ varies with e.g. oxygen background pressure, growth mode and surface coverage [18].

To monitor/measure the growth of islands, many datapoints of the island radius are needed in a timescale similar to the relaxation time $\tau$. Therefore, monitoring the growth kinetics during PLD in real-time, such as growth of islands, has to start immediately after a deposition pulse. It implies that the tip-sample approach and sample transfer time from PLD to AFM should be at least within fractions of the relaxation time scale $\tau$ in order to extract growth parameters.

Small nuclei sizes are characteristic in PLD due to a high temporal supersaturation requiring high spatial resolution in AFM. Depending on the growth conditions and oxide film material, different average island areas have been measured ex situ after deposition stopped at the minimum RHEED intensity. Ex situ AFM images 
after deposition reveal that $\mathrm{SrRuO}_{3} 2 \mathrm{D}$ islands are relative large as compared to $2 \mathrm{D}$ islands of $\mathrm{SrTiO}_{3}[17,18,33]$. Island sizes of around $150 \mathrm{~nm}^{2}$ are found for $\mathrm{SrTiO}_{3}{ }^{5}$ and $500 \mathrm{~nm}^{2}$ for $\mathrm{SrRuO}_{3}{ }^{6}$. Nuclei sizes are expected to be significantly smaller [193]. In addition, pulsed laser interval deposition has been applied to manipulate the island density and island radius in order to control the growth of $\mathrm{SrTiO}_{3}$ and superlattices of $(\mathrm{Ba}, \mathrm{Sr}, \mathrm{Ca}) \mathrm{CuO}_{2}$ [44].

Other thin film growth related parameters, for example the critical nucleation density, can be measured by monitoring the nucleation density. The nucleation density increases after every deposition pulse up to a certain critical nucleation density, mainly determined by the supersaturation of the vapor, whereafter islands start to grow. As the nucleation density is assumed to be constant in between subsequent deposition pulses, it can be measured once in between deposition pulses requiring less ambitious specifications as compared to measuring the growth of islands.

AFM images of several micrometers in size are preferred regarding statistics, but small nuclei/islands can hardly be observed or less precise due to an increase in tip speed. On the other hand, submicron AFM image sizes reduce statistics, but enable the measurement of small nuclei/islands. Determination of an island size using AFM is limited e.g. by (1) the lateral AFM resolution and (2) the tip apex. AFM images of $\leq 2 \times 2 \mu^{2}$ in size with $256 \times 256$ pixels enable the measurement of $\geq 16 \mathrm{~nm}$ island sizes. However, the average tip apex of the cantilevers used in this thesis are around $10 \mathrm{~nm}$. Therefore, the limitation in lateral resolution $\approx 10 \mathrm{~nm}$ and therefore island sizes can be measured down to $\approx 10 \mathrm{~nm}$.

Studying local nucleation and growth phenomena requires monitoring of these events at the same surface location. This requires the reposition repeatability error of the sample transfer stage to be sufficient low to follow single nuclei/islands. The required reposition repeatability error depends on the size of the monitored area. Preferably, the error is as small as possible, but should at least not exceed $\approx 10 \%$ of size of the monitored area ${ }^{7}$, which is $\leq \pm 200 \mathrm{~nm}$ of a required $\leq 2 \times 2 \mathrm{\mu m}^{2}$ AFM image.

\footnotetext{
${ }^{5}$ Deposition temperature of $850{ }^{\circ} \mathrm{C}$ used for $\mathrm{SrTiO}_{3}$ deposition. Island area rapidly decreases using lower deposition temperatures.

6 This value is found for $\approx 0.5$ monolayer. An island area of $100 \mathrm{~nm}^{2}$ was measured for $\approx 1.5$ monolayer. A deposition temperature of $700{ }^{\circ} \mathrm{C}$ was used for deposition of $\mathrm{SrRuO}_{3}$.

${ }^{7}$ Assumption is made that without exceeding this value growth of specific islands can be followed over time.
} 
Below, a summary of the side approach specifications for AFM monitoring of PLD growth kinetic processes are summarized:

- $t_{\text {approach }}<\tau \approx$ (sub)seconds.

- Sample transfer stage reposition repeatability error $\leq \pm 200 \mathrm{~nm}$.

The total side approach time is defined as $t_{\text {approach, which is the time between }}$ sample transfer from PLD to AFM position and continue AFM imaging of the surface topography. In the following section, the concept of the side approach is described.

\subsection{Side approach concept}

Figs. 4.2 (a-c) schematically illustrate the side approach concept [206]. By definition, the z-axis is perpendicular to the sample transfer plane in the x-axis. Furthermore, the sample position at the AFM is defined as position A and the sample position at the PLD as position B. In Fig. 4.2 a), the sample is located at position A. The AFM tip and sample surface are brought once into contact by a scanner coarse approach ${ }^{8}$ in the z-direction to start imaging in one of the feedback-modes. The constant force feedback can be based on cantilever deflection, amplitude, phase or frequency modulation to maintain the force setpoint by adjusting the z-piezo deformation.

The AFM is raster-scanning the tip over the sample surface to reconstruct a topography image. Raster-scanning of the AFM tip is continued during and after sample transfer from position A to position B without retracting the AFM tip in the z-direction by the coarse approach mechanism, see Fig. 4.2 b) and the constant force feedback maintains the force setpoint. This results in an AFM-tip transfer in the z-direction due to the z-piezo extension. Since there is no sample below the AFM tip, the z-piezo gets a maximum extension $Z^{*}$ as there is no tip-sample interaction possible.

After sample transfer from A to B and an additional wait time at position B for a deposition pulse, the sample is transferred back to position $\mathrm{A}^{\prime}$ and approaches the AFM tip from the side ( $\mathrm{A}^{\prime}$ is defined as the new sample position after a side approach). Close to position A', the tip-sample distance decreases during sample transfer due to the small sample angle $\alpha=1^{o}$ with respect to the $\mathrm{x}$-direction. The interaction force is maintained when the AFM tip is again "in contact" with

\footnotetext{
${ }^{8}$ Commonly used tip-sample approach.
} 


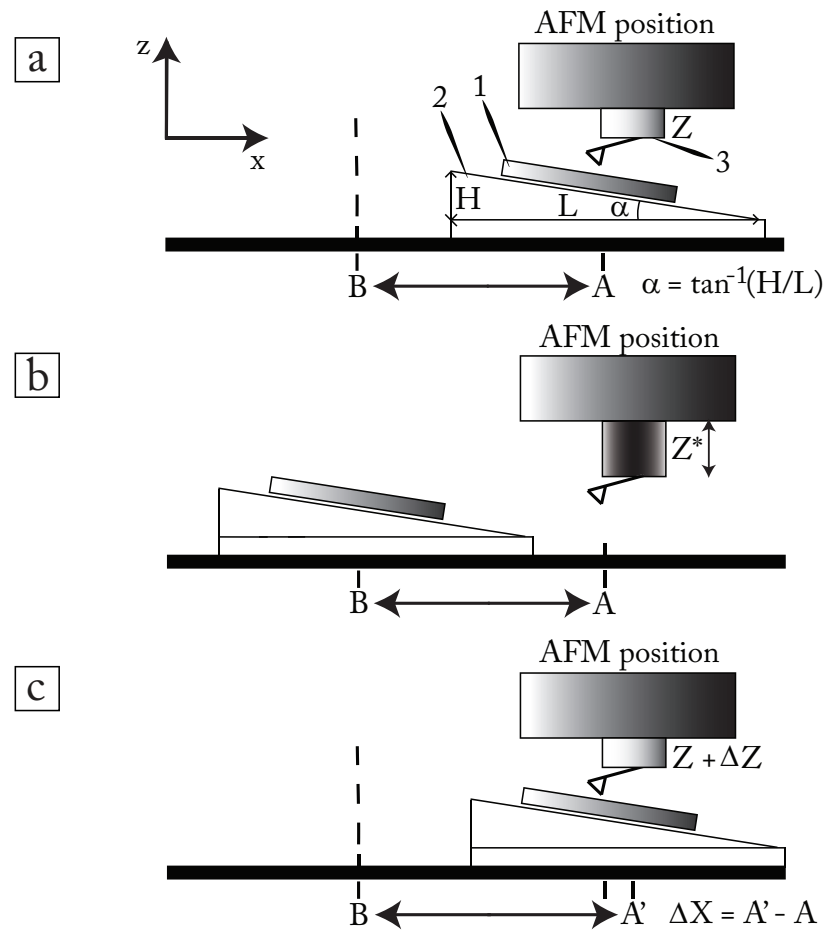

Fig. 4.2 The sample is placed under a small angle $\alpha$ (top face sample holder shaved off $\alpha$ $=1^{\circ}$ ) in order to approach from the side and reposition the AFM tip at nearly the same surface location. (a) At the initial position A, AFM surface imaging is started after using the standard tip-sample coarse approach. (b) Subsequently, the sample is transferred from position A to position B. The force feedback adjusts the z-piezo deformation to maintain the interaction force set by the force setpoint. In the case the sample is not located at position $\mathrm{A}$ or $\mathrm{A}^{\prime}$, the z-piezo has a maximum extension defined as $Z^{*}$. (c) After a wait time at position B, the sample is transferred from position B to position A'. At position A', the sample approaches the AFM tip from the side. Once the sample has approached the tip, the AFM displays the surface topography due to the restored tip-sample interaction. 
the sample surface, because the feedback mechanism maintains the same force setpoint. During the side approach, the z-piezo turns out to follow the small angle of the sample surface until the stage is decelerated. The change in z-piezo deformation $\Delta Z$ induced by the repositioning repeatability error $\Delta X$ (because of $\alpha=1^{\circ}$ ) is small compared to the z-range (typically, $1-15 \mu \mathrm{m}$ ) of the z-piezo. For instance, a repositioning repeatability error $\Delta X= \pm 1 \mu \mathrm{m}$ induces a z-piezo deformation of only $\Delta Z= \pm 17 \mathrm{~nm}$. This relation is described by:

$$
\Delta Z=\Delta X \tan (\alpha)=\Delta X \times \frac{H}{L}
$$

where $\Delta Z$ is z-piezo deformation, $\Delta X$ is the repositioning error, $\alpha$ is the angle of the sample holder, $H$ is the height of the sample holder, $L$ is the length of the sample holder, see Fig. 4.2 a).

\subsection{Design}

In the experimental setup, a home-built transfer stage was attached by an aluminum mounting bracket to the sample stage of a commercial available Bruker Dimension Fastscan AFM, see Fig 4.3 ${ }^{9}$. AFM imaging was performed in tapping mode (TM) using a Bruker FastScan-A silicon nitride cantilever/force sensor $^{10}$. The transfer stage was driven by a Nanomotion HR4 ultrasonic piezomotor (length $\times$ width $\times$ height; $42 \times 46.6 \times 15 \mathrm{~mm}^{3}$, weight $73 \mathrm{~g}$ ) having a maximum sample transfer speed of $244 \mathrm{~mm} / \mathrm{s}$ (maximum acceleration/deceleration of $4.8 \mathrm{~m} / \mathrm{s}^{2}$, stroke $43 \mathrm{~mm})^{11}$. For stage position readout, a Renishaw RGH25F UHV optical encoder with a resolution of $5 \mathrm{~nm}$ was integrated. A FlexDC nanomotion controller (RS232 - software SC Shell 9.01) was used in order to control the HR4 nanomotion motor.

Two samples were prepared in order to test and demonstrate the side approach performance. Sample (I) was a silicon substrate with a titanium nanocross on top (wire dimensions: length $\times$ width $\times$ height; $10000 \times 100 \times 10 \mathrm{~nm}^{3}$ ) fabricated by e-beam lithography. Sample (II) was a $\mathrm{TiO}_{2}$-terminated $\mathrm{SrTiO}_{3}(001)$ substrate with $0.4 \mathrm{~nm}$ height substrate steps [19].

\footnotetext{
${ }^{9}$ A HS-AFM is used to show the applicability of combining the side approach method with real-time surface monitoring.

${ }^{10}$ Resonance frequency $\approx 1.4 \mathrm{MHz}, 27 \mu \mathrm{m}$ long triangular shaped, force constant $17 \mathrm{~N} / \mathrm{m}$ and tip radius $5 \mathrm{~nm}$.

${ }^{11}$ An UHV $\geq 10^{-9}$ mbar compatible HR4 nanomotion version was selected to meet the typical oxide PLD pressures ranging from $10^{-6}-10^{-1}$ mbar $\mathrm{O}_{2}$.
} 


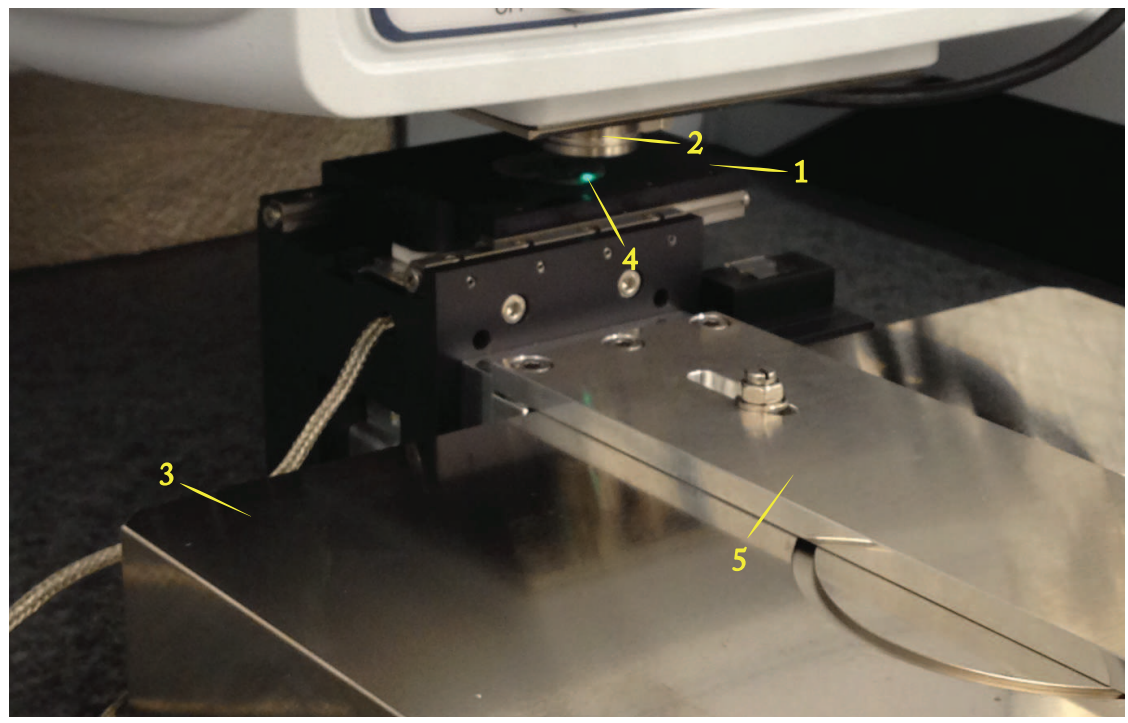

Fig. 4.3 Experimental setup with key components labeled. (1) Home-built transfer stage, (2) AFM scanner, (3) sample stage Bruker Dimension Fastscan, (4) $1^{\circ}$ angle sample plate suited for a $5 \times 5 \mathrm{~mm}^{2}$ sample on top, (5) aluminum mounting bracket to connect the home-built transfer stage to the Fastscan sample stage.

Sample (I) was used to demonstrate the ability to monitor the same surface location after subsequent side approaches. The center of the nanocross was followed during repetitive side approaches to measure the repositioning repeatability error of the transfer stage. Sample (II) was used to test any additional tip wear after subsequent side approaches. AFM imaging was performed using a line rate $f$ of respectively $25.3 \mathrm{~Hz}$ and $27.9 \mathrm{~Hz}$ during imaging of a surface area of $2 \times 2 \mathrm{\mu m}^{2}$ with $256 \times 256$ pixels. During the measurements, the AFM scan direction was always from top to bottom. The wait time between the subsequent sample transfers from position $\mathrm{B}$ to position $\mathrm{A}$ and vice versa was set to $10 \mathrm{~s}$ as a typical time used in between deposition pulses. 


\subsection{Performance}

In this section, the performance of the side approach is demonstrated using the designed sample transfer stage. First, the AFM images before, during and after the side approach are shown. Thereafter, the AFM scanlines before, during and after the side approach are presented. Furthermore, the reposition repeatability error of the designed transfer stage is determined. This section ends by showing the results on possible tip wear caused by the side approach.

\subsubsection{Continuous AFM imaging during the side approach}

In Fig. $4.4 \mathrm{a}$ ), a single $2 \times 2 \mathrm{\mu m}^{2}$ AFM image is depicted of continuous AFM imaging of the sample surface before sample transfer from position A to position B. Continuous AFM imaging was started after bringing the AFM tip into contact with the sample surface using the coarse approach. The nanocross center was placed nearly in the center of the $2 \times 2 \mathrm{\mu m}^{2}$ AFM image in order to follow the repositioning repeatability error of the transfer stage.

After continuous AFM imaging of the nanocross sample surface at position A, the raster-scanning of the AFM tip was continued during and after retracting the sample from the AFM tip by the sample transfer from position A to position B, visible in Fig. 4.4 b). The sample positions A, A' and B in time are represented by A, A' and B in Figs. 4.4 (b-c). The sample surface topography is displayed by the $\mathrm{AFM}$ at position $\mathrm{A}$, while during the time the sample is not located at position $\mathrm{A}$ or A' the tip-sample interaction force is lost and no surface topography is obtained (the latter is visible in the bottom part of the AFM image in Fig. 4.4 b)). Retracting the sample from the AFM tip is fast as there are no or only a few scanlines representing the z-piezo extension, by following the sample surface, during sample transfer from position A to B (Fig. 4.4 b)). In between sample retracting from and approaching the sample to the AFM tip from the side, the AFM displays images containing noise, since there is no tip-sample interaction force.

After the wait time at position B, the sample was transferred to position A' and the sample approaches the AFM tip from the side. Fig. $4.4 \mathrm{c}$ ) shows an AFM image during the sample transfer from position B to position A' and the side approach of the sample to the AFM tip at position A' (red rectangle marker surrounds AFM scanlines during the side approach, see Figs. $4.4 \mathrm{c})$ ). At the top part of the AFM image in Fig. $4.4 \mathrm{c}$ ), the sample is located at position B and therefore the AFM displays no sample surface topography. Between B and A' in Fig. 4.4 c), the sample is transferred back to the AFM position and approaches the AFM tip 
AFM nanocross

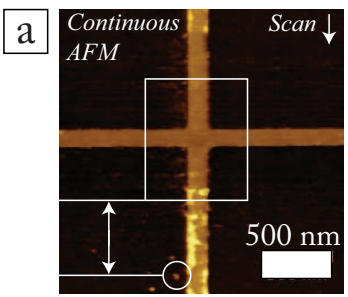

$\mathrm{b}$

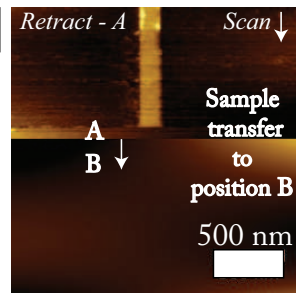

c
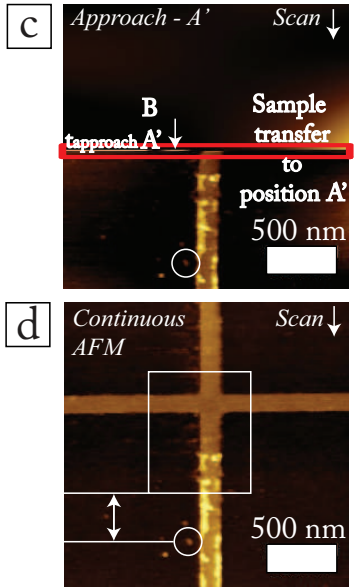

AFM tip \& sample position

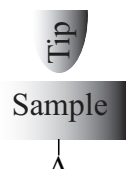

A

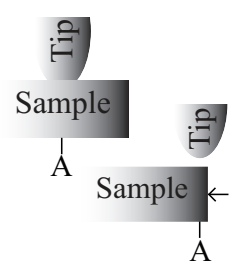

()ㅡ
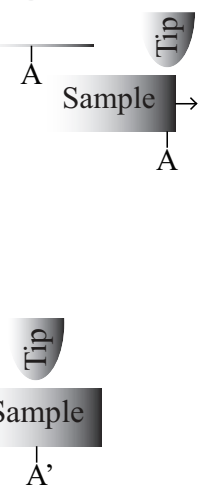

AFM SrTiO3
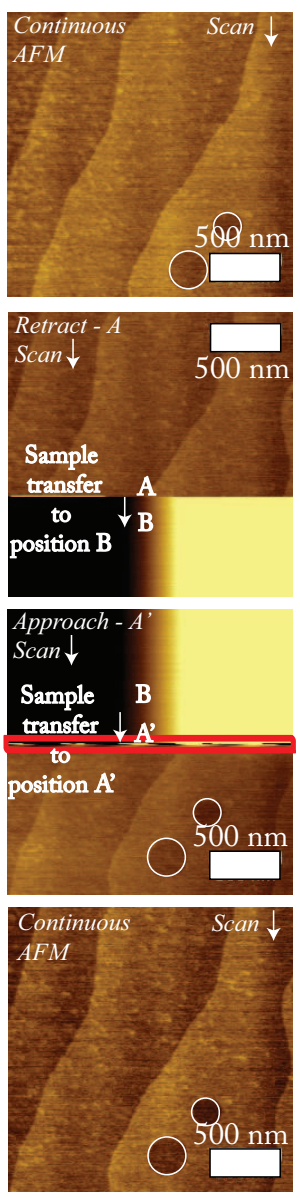

Fig. 4.4 Ex situ TM-AFM $2 \times 2 \mu^{2}, 256 \times 256$ pixels images of the nanocross (line rate $f=25.3 \mathrm{~Hz}$ ) and $\mathrm{SrTiO}_{3}(001)$ sample (line rate $f=27.9 \mathrm{~Hz}$ ) using the side approach method (a) before sample transfer from position A to position B (the center of the nanocross was positioned in the center of the white rectangle marker, circle had a fixed location on the sample surface), (b) during retraction of the AFM tip at position A by sample transfer from position A to position B, (c) during sample transfer from position B to position A', the sample is approaching the AFM tip from the side and (d) continuous AFM imaging after 25 repetitive side approaches (center nanocross moved away from the center of the marker, circle marker moved closer to the rectangle marker). 
from the side.

During approach, the sample surface comes close to the AFM tip and the z-piezo contracts such that the AFM tip follows the angle of the sample surface during sample transfer. At that moment, the tip-sample interaction is restored and the force setpoint is maintained by the force feedback adjusting the z-piezo deformation. However, before the sample surface topography is displayed at position A', artificial scanlines ${ }^{12}$ are observed in the AFM image of Fig. 4.4 c).

After the artificial scanlines, the sample surface topography is displayed and is visible in the bottom part of the AFM image in Fig. $4.4 \mathrm{c}$ ). The AFM tip is now located at a new sample position $A^{\prime}$ due to the repositioning repeatability error of the stage $\Delta X$. As a consequence, the center of nanocross slightly moves out of the center of the AFM image. Using the same scan direction, the AFM image in Fig. $4.4 \mathrm{c}$ ) is mirrored to the AFM image in Fig. 4.4 b) as the moment of tip-sample interaction is mirrored in the vertical direction of the AFM images.

Fig. 4.4 d) shows a single AFM image of continuous AFM imaging of the sample surface after 25 times of repetitive side approaches. The center of the nanocross has slightly moved out of the center of the $2 \times 2 \mu^{2}$ AFM image (the nanocross center shifted with respect to the white rectangle marker in Figs. 4.4 a) and d)).

Thereafter, the measurements were repeated after replacing the nanocross sample by the $\mathrm{SrTiO}_{3}(001)$ sample to test the side approach performance on an atomically flat surface (shown in the right panel of Fig. 4.4). The $\mathrm{SrTiO}_{3}(001)$ sample was chosen by the fact that it is the most commonly used perovskite oxide substrate for PLD grown thin films. Conclusions based on the nanocross sample can be extrapolated on atomically flat samples typically used in PLD experiments, such as $\mathrm{SrTiO}_{3}(001)$, see Figs. 4.4 (a-d).

\subsubsection{Side approach scanline(s) analysis}

In the AFM images of Fig. $4.4 \mathrm{c}$ ), artificial scanlines are present after the side approach, resulting in a time delay $t_{\text {delay }}$ before the AFM displays the surface topography in the image ${ }^{13}$. AFM scanlines recorded during side approaching the sample to the AFM tip are indicated by the red rectangular box in Fig. $4.4 \mathrm{c}$ ), and the height profiles of the corresponding scanlines are analyzed.

Fig. 4.5 a) shows a zoom-in on the AFM image of the red rectangular marker

\footnotetext{
${ }^{12}$ Here, artificial scanlines refer to AFM scanlines recorded after stage deceleration at position A' having tip-sample interaction without displaying the sample surface topography.

13 The AFM acquisition time of the artificial scanlines is assumed to be equal to the additional time loss after the side approach and displaying the surface topography with AFM.
} 
shown in Fig. $4.4 \mathrm{c}$ ) during one of the repetitive side approaches. In addition, the corresponding height profiles of the AFM scanlines 161 - 164 are shown in Fig. 4.5 b) ${ }^{14}$. Scanline 161 has a color gradient from dark brown to light brown due to the mean plane subtraction used in image representation. Subtraction of the mean plane is done in order to correct for the surface tilt displayed in the surface topography caused by the small angle $\alpha=1^{\circ}$ (see Fig. 4.2) of the sample surface $^{15}$. During acquisition of scanline 161, the sample has not approached the AFM tip from the side, but the sample is most probably in close vicinity to the AFM tip.

A large color modulation is observed in scanline 162 related to a large height modulation (Fig. 4.5 (a-b)). From the height profile of scanline 162, it seems that these height oscillations are periodic in time. The scanline recorded during stage deceleration has a gradient in its height profile due to the small sample tilt of $1^{\circ}$. In scanline 162 , the transfer stage is most likely decelerating/decelerated at position A'. As the height profile represents the contraction/extension of the z-piezo, the z-piezo oscillates during scanline 162 . Scanline 163 shows part of the nanocross, but there is still a small height modulation visible. The oscillation amplitude of the z-piezo is small compared to the previous scanline 162. In scanline 164, AFM imaging is continued without the observation of vibrational (z-piezo) oscillations.

Some vibrational modes are recorded by the AFM as the force feedback maintains the tip-sample distance. A Fast Fourier Transformation (FFT) is applied to find the vibration frequencies observed in scanline 162, which is compared to the scanlines 161, 163 and 164. Vibration frequencies of $660 \mathrm{~Hz}$ and $4.65 \mathrm{kHz}$ are obtained in scanline 162 and not observed in the scan lines 161, 163 and 164. Therefore, it is assumed that these frequencies correspond to the resonance frequencies of the sample transfer stage. In addition, the vibrational frequencies do not match with the pixel clock frequency ${ }^{16}$ of $13.0 \mathrm{kHz}$ used during the measurement, whereas it should appear in all scan lines if this were an issue.

A time delay $t_{\text {delay }}$ of $0.08 \pm 0.04 \mathrm{~s}$ and $0.11 \pm 0.04 \mathrm{~s}$ is measured using the nanocross sample and $\mathrm{SrTiO}_{3}(001)$ sample, respectively. A different $t_{\text {delay }}$ is obtained because of discretisation and the different line rate used during the AFM measurement of the nanocross sample $(f=25.3 \mathrm{~Hz})$ and $\mathrm{SrTiO}_{3}(001)$ sample $(f=27.9 \mathrm{~Hz})$. From the HR4 motor motion profile in the software SC Shell 9.01,

\footnotetext{
${ }^{14}$ In this chapter, AFM images contain 256 scanlines in the vertical scan direction.

${ }^{15}$ Mean plane subtraction tilts the height profiles of the scanlines such that the average angle of the scanline height profile becomes zero with respect to a horizontal height profile. The number of scanlines with or without tip-sample interaction are similar, but with an opposite sign of the angle.

${ }^{16}$ Pixels per line $\times$ line rate $\times 2$ (latter for trace and retrace) .
} 

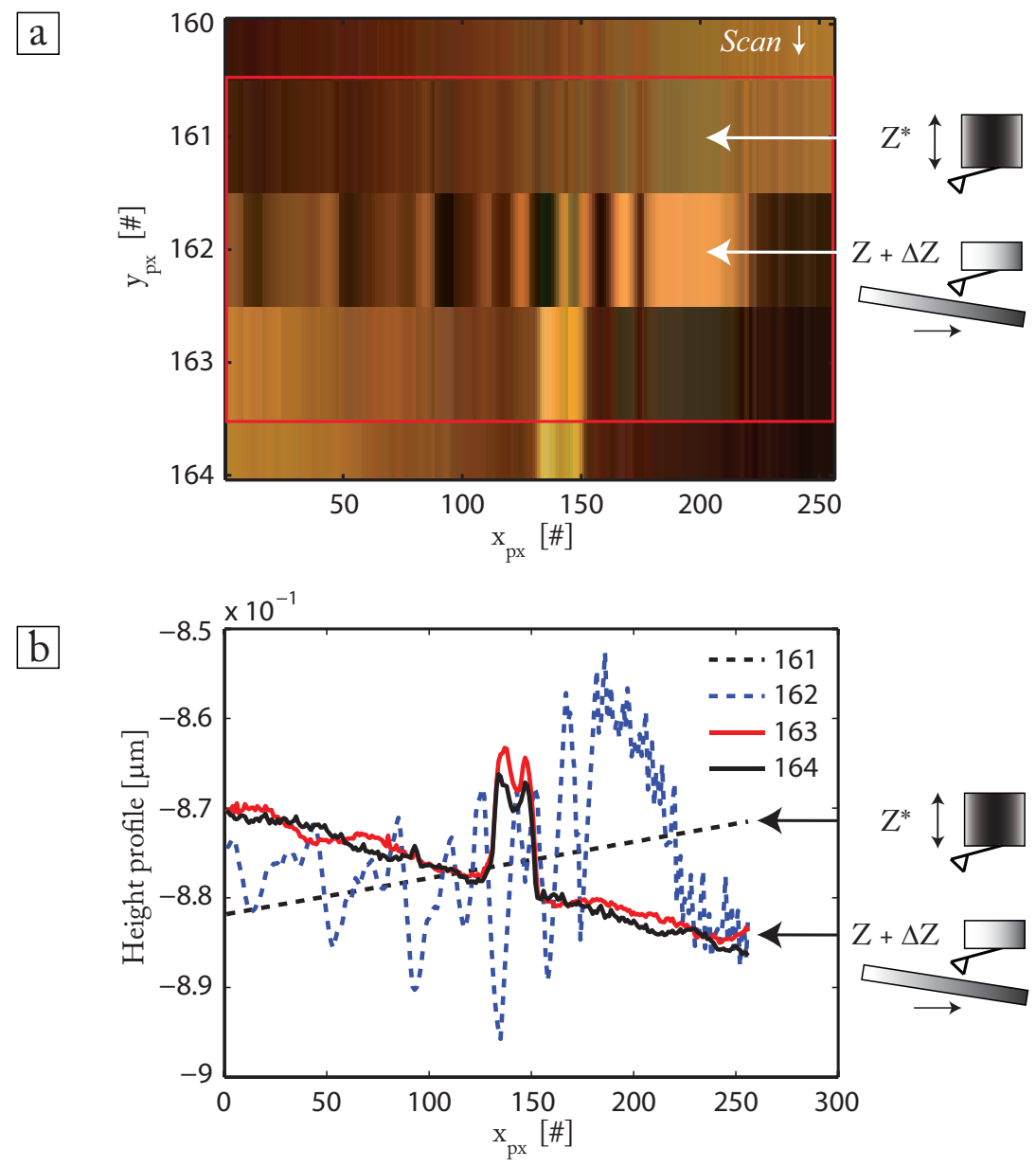

Fig. 4.5 Height profiles before, during and after the side approach (a) zoom-in AFM image (red rectangle marker Fig. 4.4 c)) during one of the side approaches (see Fig. $4.4 \mathrm{c}$ ) for a typical AFM image during side approach) and (b) the height profiles of the scanlines 161-164 during the side approach. Scanline 161 is recorded during sample transfer and the sample is in close vicinity to the AFM tip, scanline 162 during tip-sample interaction and scanline 163 during the time the AFM poses to image the height profile of the nanocross sample (nanocross height $10 \mathrm{~nm}$ ). The scanlines 162 and 163 show vibrational modes. Displaying the nanocross sample surface topography is continued from scanline 164. $Z^{*}$ means a fully extended z-piezo as there is no tip-sample interaction, where $Z$ $+\Delta Z$ means that the z-piezo is contracted/extended within the limits of the z-range as there is tip-sample interaction since the sample is positioned below the AFM tip. The angle of the height profiles is indicative for when the tip-sample interaction is restored during the side approach. 
a sample transfer (position $\mathrm{B} \rightarrow$ position A) time of $0.22 \mathrm{~s}$ (depends on the user settings) was measured over $35 \mathrm{~mm}$. Therefore, $t_{\text {approach }}$ can be at least reduced to $\approx 0.3 \mathrm{~s}$. The quality factor of the transfer stage is most likely an important parameter to decrease $t_{\text {delay }}$. Furthermore, the bandwidth of the AFM feedback seems to be a crucial parameter to reduce the side approach time $t_{\text {approach }}{ }^{17}$.

\subsubsection{Sample location reposition repeatability error}

The nanocross center was followed to determine the repositioning repeatability error of this transfer stage. Fig. 4.6 shows results of the reposition repeatability error measurements. A repositioning repeatability error within $\Delta X= \pm 60 \mathrm{~nm}$ is measured after correction for thermal drift. Several measurements have been performed to confirm a temperature increase of the transfer stage. The drift correction is performed by using a moving average filter. The drift of the Bruker Dimension Fastscan was minimal (few $\mathrm{nm} / \mathrm{min}$ ) and assumed to be negligible in these side approach experiments.

\subsubsection{AFM tip wear}

Continuously TM-AFM imaging of a sample surface affects the AFM image resolution due to an AFM tip apex increase [207]. This loss in spatial AFM resolution makes it harder to resolve small single islands and their growth. To study any additional loss in resolution caused by the side approach, no tip wear should occur caused by AFM surface imaging. AFM tip wear during the side approach can be expected based on the fact that the maximum sample transfer speed of $244 \mathrm{~mm} / \mathrm{s}$ is much faster than the AFM tip speed of $\approx 100 \mu \mathrm{m} / \mathrm{s}$ used in these experiments. Moreover, scanline 162 in Fig. 4.5 shows that the AFM tip oscillates, which might cause AFM tip wear.

The results to test tip wear after subsequent side approaches are shown in Fig. 4.7. In these experiments, 25 repetitive side approaches (similar to Fig. 4.4) were performed in order to study any tip wear caused by the side approach. A single $2 \times 2$ $\mu^{2}$ AFM image $(f=27.9 \mathrm{~Hz}, 256 \times 256$ pixels $)$ is depicted in Fig. 4.7 a) showing the unit cell terraces of the $\mathrm{SrTiO}_{3}(001)$ sample. Continuous AFM imaging was started after the coarse approach.

In Fig. 4.7 b) an AFM image (same settings as Fig. 4.7 a)) is depicted of the

\footnotetext{
${ }^{17}$ An oscillating piezo was used to test the maximum speed of the side approach using the LPM feedback controller. These experiments reveal that a five times faster sample transfer can be used in order to approach the AFM tip from the side.
} 


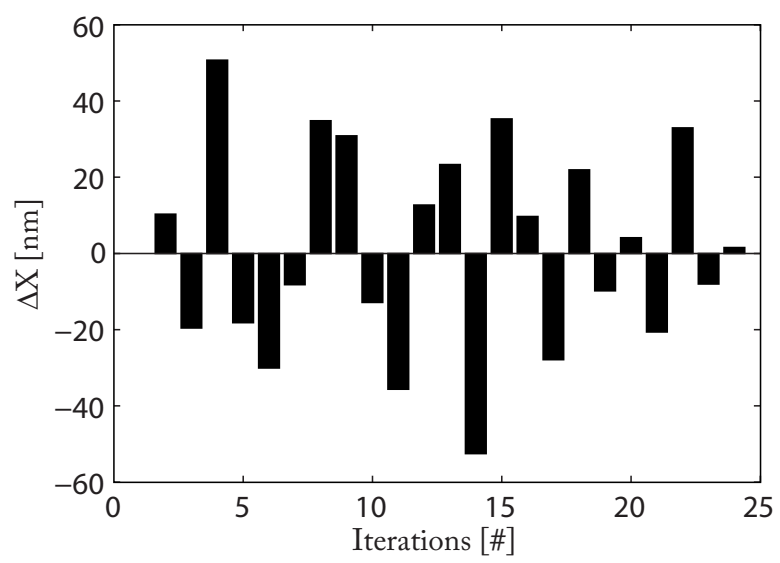

Fig. 4.6 Repositioning repeatability error of the home-built transfer stage is within $\Delta X$ $= \pm 60 \mathrm{~nm}$ measured over 25 side approaches after correction for thermal drift. Measured thermal drift of the home-built transfer stage is $1.5 \mu \mathrm{m} /{ }^{\circ} \mathrm{C}$ in air.

$\mathrm{SrTiO}_{3}(001)$ sample surface after 25 subsequent side approaches. The AFM resolves the unit cell terraces after 25 side approaches. In addition, the locations of the vacancy islands in the terrace, see Fig. 4.7 a) and Fig. 4.7 b), confirm the small repositioning repeatability error. The unit cell steps $(0.4 \mathrm{~nm})$ of the terraces can still be observed in the height profile of Fig. $4.7 \mathrm{~d}$ ). The measured RMS surface roughness remained $\sigma_{\mathrm{RMS}}=0.18 \mathrm{~nm}$ after 25 side approaches and was similar to the condition where 25 coarse approaches were applied, which demonstrates that there is no significant resolution degradation using the side approach method. 

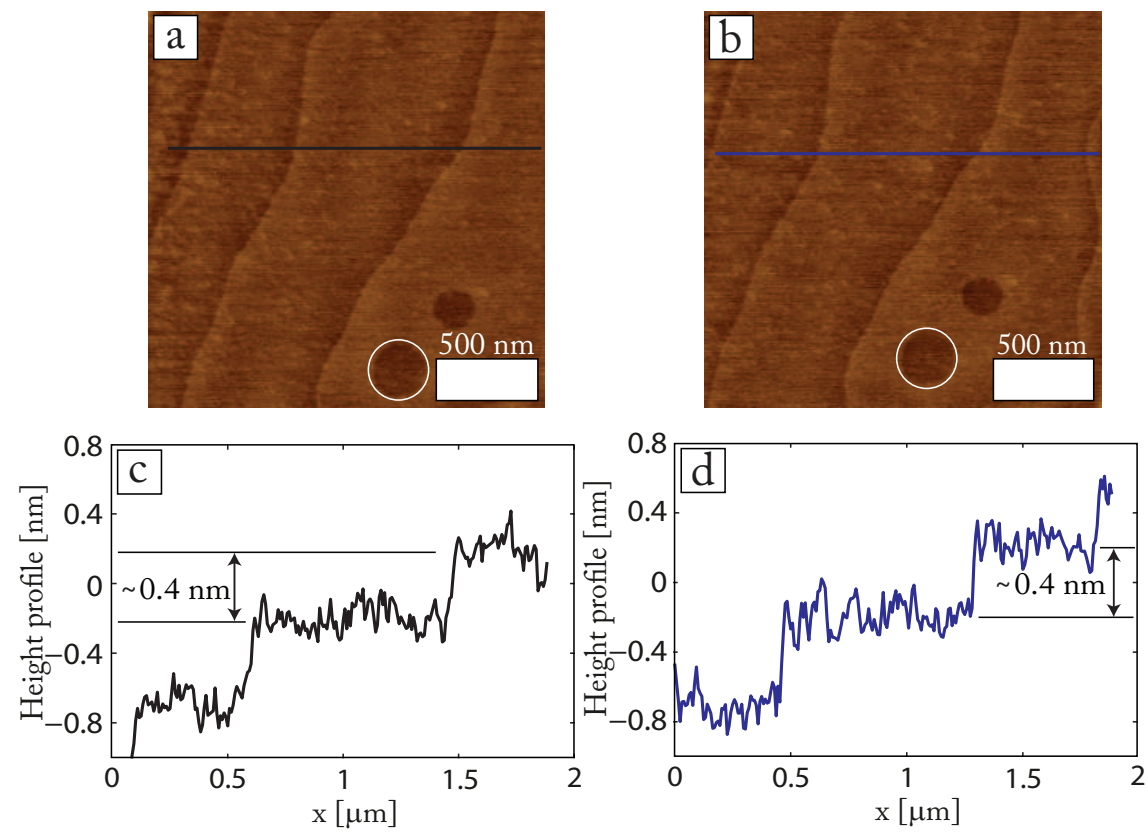

Fig. 4.7 Ex situ TM-AFM $2 \times 2 \mu^{2}, 256 \times 256$ pixels, $f=27.9 \mathrm{~Hz}$ images of the $\mathrm{SrTiO}_{3}(001)$ sample (a) before the side approach (circle marker surrounds vacancy island to follow location during side approach) and (b) after 25 repetitive side approaches, (c) height profile of the $\mathrm{SrTiO}_{3}(001)$ surface before the side approach experiments. Step resolution $(0.4 \mathrm{~nm})$ was still resolved in the $(\mathrm{d})$ corresponding height profile (blue line Fig. $4.7 \mathrm{~b})$ ) of the AFM image after 25 repetitive side approaches. The $\sigma_{\mathrm{RMS}}=0.18 \mathrm{~nm}$ remained after 25 side approaches. 


\subsection{Conclusions}

In this chapter, it has been demonstrated that the side approach time $t_{\text {approach }}$ can be reduced from several seconds to subseconds using a faster transfer stage. From the motor motion profile, it is deduced that a $t_{\text {approach }}$ of $\approx 0.3 \mathrm{~s}$ is feasible using a PLD - AFM position separation of $\approx 35 \mathrm{~mm}$ as used in the design. An vibrational decay time $t_{\text {delay }}$, which is a part of $t_{\text {approach }}$, is observed most likely due to the resonating sample transfer stage after deceleration. A $t_{\text {delay }}$ is found of $0.08 \pm 0.04 \mathrm{~s}$ and $0.11 \pm 0.04 \mathrm{~s}$ using (two) different samples, respectively.

A repositioning repeatability error of $\pm 60 \mathrm{~nm}$ is achievable using the designed transfer stage, which enables monitoring the same surface location after back and forth sample transfer. As the height resolution and image quality are not degraded after 25 repetitive side approaches, it is concluded that AFM tip wear is presumably negligible. In total, over hundred repetitive side approaches are performed without observation of additional tip wear using the same cantilever.

In summary, it is demonstrated that the side approach time can be reduced using a faster transfer stage. The achievable side approach time is inprinciple limited by the bandwidth of the AFM feedback. The side approach can become widely used as it enables sample modification and subsequent atomic force microscopy imaging at a specific surface location. For PLD growth studies, this opens the opportunity to real-time monitor growth kinetics using HS-AFM. 


\section{Part III}

\section{Experiments \& Models}





\title{
Chapter 5
}

\section{Energetics of vicinal perovskite oxide surfaces}

\begin{abstract}
The surface energetics plays an important role in the perovskite oxide growth front evolution during pulsed laser deposition (PLD). During growth, atoms diffuse and their hopping probability scales with i.e. the nearest neighbor interaction energy, where the latter can be determined from surface step meandering. In this chapter, the meandering of equilibrated step-edges at oxide surfaces is analyzed using ex situ atomic force microscopy (AFM). The kink formation energy of perovskite oxide unit cells is extracted from small scale meandering and amounts $0.10 \mathrm{eV}$ per unit cell. It results in a nearest neighbor interaction energy of $0.21 \pm 0.01 \mathrm{eV}$ considering a simple square lattice with isotropic interactions. On a larger length scale, a triangular step undulation is observed with a wavelength of $\approx 2100$ unit cells. This step undulation is caused by strain relaxation along the mean step direction and is estimated to be $24 \mathrm{meV}$ per unit cell. These experiments reveal that the energetics of perovskite oxide surfaces is mainly governed by the local oxygen coordination. The comparable surface energetics of different perovskite oxides might result into analogous growth behavior and a similar behavior of the oxygen exchange and defect formation in perovskite oxide heterostructures at surfaces and interfaces.
\end{abstract}

W.A. Wessels, G. Koster, H.J.W. Zandvliet and G. Rijnders, "Energetics of vicinal perovskite oxide surfaces", under review. 


\subsection{Introduction}

Surface steps have a large influence on both equilibrium (e.g. thermal roughening and faceting) and non-equilibrium (e.g. epitaxial growth and etching) surface processes. Knowledge on step-related parameters is of utmost importance for describing and understanding these surfaces. During crystal cutting, the creation of step-edges on a crystal surface is unavoidable as cutting the crystal exactly along the low Miller indices planes is nearly impossible. The miscut angle of the vicinal substrate determines the average distance between the crystal layer height surface steps. The formation of steps usually introduces surface stress influencing the step-edge morphology. Most of the step-related surface studies have been focused on $\mathrm{Si}$ as a model system, because of its technological importance [208, 209].

Perovskite oxides, with chemical formula $\mathrm{ABO}_{3}$, are often used as substrates and/or thin films. In the closed packed perovskite oxide unit cell, an oxygen octahedra is surrounded by four alkaline (rare) earth elements and a transition metal element in the center, where this $\mathrm{ABO}_{3}$ unit cell is the elementary building block of the crystal. Such building blocks lead to well-defined steps at the surface, which can be resolved by microscopy techniques, such as scanning probe microscopy (SPM) [31]. Atomic force microscopy (AFM) [30] has the advantage that it can examine the lateral distribution of the building blocks perpendicular to the step of solid surfaces independent of its band gap. Most perovskite oxides have large band gaps and thus no tunneling current is flowing during scanning tunneling microscopy (STM).

Perovskite oxide crystal cutting gives rise to a surface, where long-range Coulomb repulsion plays a significant role in the type and strength of surface bonds. The long-range Coulomb interaction is more dominant in perovskite oxides compared to semiconductor as screening becomes less efficient [67]. Analyzing surface energetics of perovskite oxides seems to be rather complicated compared to semiconductor surfaces as for the latter the strength of long-range Coulomb interaction $(\approx 10 \%$ of short range interaction energy for Ge) is mostly neglected [210, 211]. For semiconductor surfaces, the interaction energy between nearest neighbor atoms is typically extracted from its kink formation energy considering a square lattice with isotropic nearest neighbors [208, 212]. However, this isotropic nearest neighbor interaction assumption has been applied in the kinetic Monte Carlo (KMC) simulations in order to predict perovskite oxide thin film growth modes or to study the influence of/as function of pulsed laser deposition (PLD) growth parameters [213].

Several studies show that the isotropic nearest neighbor interaction assumption is plausible for simulating perovskite oxide thin film growth as KMC simulations 
match with their observations in growth experiments $[17,18,58,148,213]$. Currently, perovskite oxide PLD thin film growth is simulated by iterative selection of energetic parameters, such as the nearest neighbor interaction energy $E_{\mathrm{N}}$. Literature reports a wide spread in nearest neighbor interaction energy values between perovskite oxide unit cells of $E_{\mathrm{N}}=0.25-0.6 \mathrm{eV}$ applied in KMC simulations $[17,18,58,148,213]$. Reciprocal diagnostic tools, i.e. reflection high-energy electron diffraction (RHEED), have been used to extract the activation barrier for diffusion $E_{\mathrm{A}}$, but are not suitable to determine $E_{\mathrm{N}}$ between perovskite oxide unit cells [18]. STM studies on semiconductors reveal that energetic parameters, such as $E_{\mathrm{N}}$, can be determined from step meandering analysis on vicinal surfaces in thermodynamic equilibrium [208, 210].

Thermal roughening of step-edges occurs due to formation of kinks, resulting in meandering of step-edges [214]. Kinks can point towards the lower or upper terrace. The creation of these kinks costs energy since bonds have to be broken. This kink formation energy $E_{\text {kink }}$ can be extracted from Boltzmann statistics assuming the surface is in equilibrium. A surface reaches its equilibrium structure by prolonged annealing at elevated temperatures. During annealing, atomic bonds can be broken resulting in atom diffusion to more energetically favorable positions. Eventually, the surface seeks its configuration with the lowest free energy. Therefore, the meandering of step-edges is governed by the step free energy.

Step meandering analysis has been applied to extract surface energetics of semiconductors in last two decades [208, 210, 212, 214]. Here, it is described how perovskite oxide steps behave and step-related energetic parameters can be extracted from AFM images of vicinal surfaces in thermodynamic equilibrium. It will be shown that energetic values of perovskite oxides can be extracted from surface step meandering analogue to semiconductors. Furthermore, the surface energetics of perovskite oxide surfaces is most likely governed by its local oxygen coordination. This study is unique in the field of complex oxides as it is a quantitative study to extract energetic parameters using AFM in contrast to qualitative stepped surface AFM studies reported in literature so far [215, 216, 217].

This chapter continues by describing the perovskite oxide surface, the perovskite oxide crystal structure and typical methods used to extract the energetics. In the subsequent section, the experiment in order to measure the kink energy at perovskite oxide surfaces is explained followed by an overview of the used methods. Thereafter, the obtained results for various oxide surfaces are shown and discussed. In the final section, the conclusions and recommended experiments are presented. 


\subsection{Perovskite oxide surface energetics}

In this section, the perovskite oxide $\left(\mathrm{ABO}_{3}\right)$ surface is described and the (relevant) literature regarding studies to extract perovskite oxide surface energetic parameters using RHEED is briefly summarized. Furthermore, the determination of the perovskite oxide energetic parameters is discussed.

\subsubsection{The (pseudo)cubic unit cell of $\mathrm{ABO}_{3}(001)$}

Most $\mathrm{ABO}_{3}$ crystals can not be considered as an ideal cubic unit cell as they are slightly distorted. Often this is due to the deviation of the ideal ionic radii of the cations. An estimation for the degree of distortion and stability of the perovskite oxide can be calculated by the Goldschmidt's tolerance factor:

$$
t_{\mathrm{ol}}=\frac{\left(r_{\mathrm{A}}+r_{\mathrm{O}}\right)}{2\left(r_{\mathrm{B}}+r_{\mathrm{O}}\right)}
$$

An ideal $\mathrm{ABO}_{3}$ has an tolerance factor of $t_{\mathrm{ol}}=1$. Most stable $\mathrm{ABO}_{3}$ materials exist with $0.75<t_{\mathrm{ol}} \leq 1$, while the oxygen octahedra might be tilted. The cubic $\mathrm{SrTiO}_{3}(001)_{c}$ has been chosen often as substrate material and has a $t_{\mathrm{ol}} \approx 0.86$.

The $\mathrm{ABO}_{3}$ unit cell exhibits a stack with alternating planes of $\mathrm{AO}$ and $\mathrm{BO}_{2}$ in the [001] direction ${ }^{1}$, see Fig. 5.1 a). Both layers appear at the surface in nearly equal proportion after surface cutting such that both universal step heights, half unit cell of $\approx 0.2 \mathrm{~nm}$ and single unit cell of $\approx 0.4 \mathrm{~nm}$, can be expected at the surface (mixed termination). However, many studies show that achieving atomic growth control of perovskite oxide films requires vicinal $\mathrm{ABO}_{3}$ surfaces with unit cell height surface steps of $0.4 \mathrm{~nm}$ having either single termination. Wet-etching together with prolonged annealing has been used successfully in order to achieve a single terminated surface of $\mathrm{BO}_{2}$ resulting in a unit cell height steps [19, 20]. Besides the $\mathrm{BO}_{2}$ termination approach, a different annealing procedure is proposed to achieve $\mathrm{SrO}(\mathrm{AO})$ termination in the case of $\mathrm{SrTiO}_{3}(001)_{c}$ (subscript c denotes cubic) [218].

For orthorhombic $\mathrm{ABO}_{3 o}$ substrates (subscript o denotes orthorhombic), the cubic $\mathrm{ABO}_{3 c}$ unit cell is distorted to become $\mathrm{ABO}_{3 p c}$ (subscript pc denotes pseudocubic). From an orthorhombic unit cell, the pseudo-cubic $\mathrm{ABO}_{3 p c}$ unit cell existing of stacked alternating planes of $\mathrm{AO}$ and $\mathrm{BO}_{2}$ can be found in a different crystal

\footnotetext{
${ }^{1}$ Most perovskite oxide thin film growth studies are conducted on substrates with alternating planes of $\mathrm{AO}$ and $\mathrm{BO}_{2}$ in the [001] direction.
} 

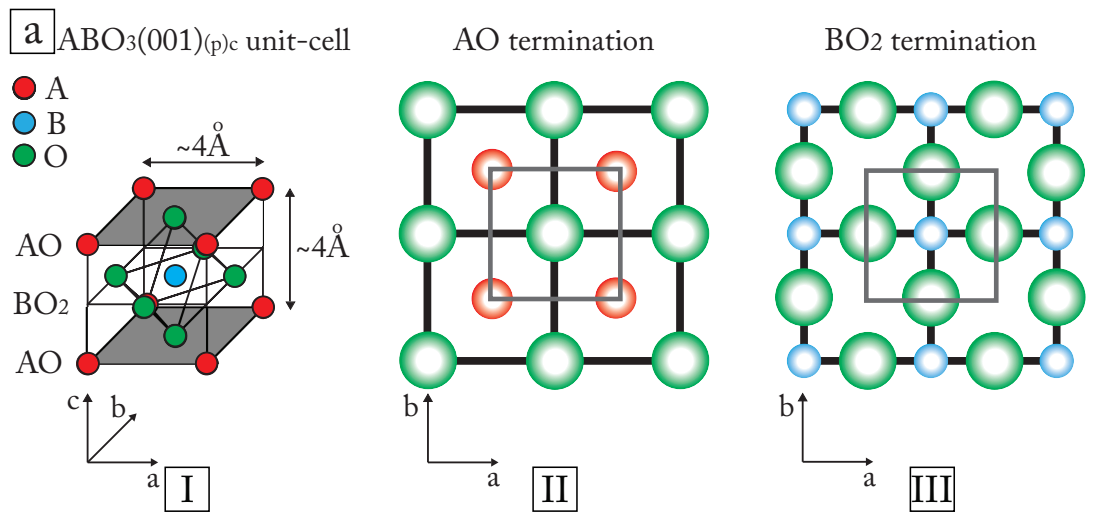

b Orthorombic(o)/ pseudo-cubic(pc) $\mathrm{ABO}_{3}$ unit-cell orientation
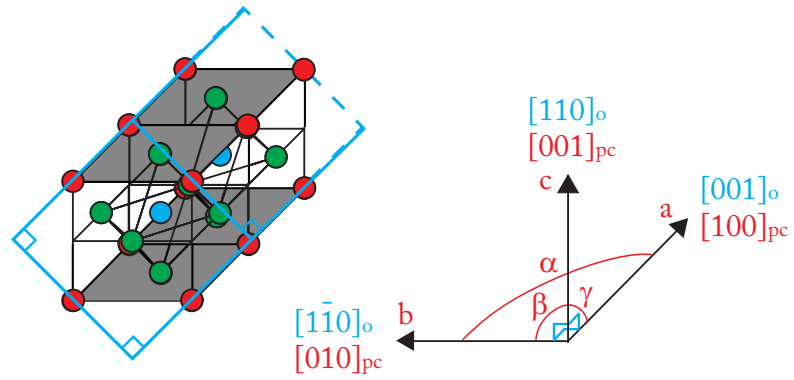

Fig. 5.1 Schematic illustration of the perovskite oxide $\left(\mathrm{ABO}_{3}\right)$ unit cell with the possible terminating planes. $\mathrm{O}, \mathrm{A}, \mathrm{B}$ ions are ordered from large to small in size, a) (I) $\mathrm{ABO}_{3}$ unit cell exist of stacked alternating planes of $\mathrm{AO}$ and $\mathrm{BO}_{2}$ (II) $\mathrm{AO}$ termination plane at the surface (III) $\mathrm{BO}_{2}$ termination plane at the surface, b) pseudo-cubic unit cell orientation with respect to the orthorhombic crystal orientation of slightly distorted $\mathrm{ABO}_{3}$ unit cell.

orientation, see Fig. 5.1 b). Despite the different crystal structure/orientation of $\mathrm{ABO}_{3}(110)_{o}$, the same terminating plane can exist such that the oxygen coordination at the surface is similar to $\mathrm{ABO}_{3}(001)_{c}$ after cutting. For example, the orthorhombic $\mathrm{DyScO}_{3}(110)_{o}$ has the $\mathrm{DyScO}_{3}(001)_{p c}$ pseudocubic equivalent.

\subsection{2 $\quad \mathrm{ABO}_{3}$ surface energetics obtained by RHEED}

Many studies regarding the measurement of $\mathrm{ABO}_{3}$ surface energetic parameters used RHEED to extract the values $[18,58]$. For example, the activation energy barrier for diffusion $E_{\mathrm{A}}$ can be calculated by measuring the single pulse relaxation time $\tau$ of the RHEED specular intensity after a single deposition pulse [17]. From the single pulse relaxation time $\tau$, the activation barrier for diffusion $E_{\mathrm{A}}$ can be 


\begin{tabular}{c|cccc}
\hline $\mathrm{ABO}_{3}$ film/substrate & $E_{\mathrm{A}}[\mathrm{eV}]$ & $E_{\mathrm{D}}[\mathrm{eV}]$ & $E_{\mathrm{N}}[\mathrm{eV}]$ & Reference \\
\hline STO/STO (RHEED) & $2.2 \pm 0.2$ & & & {$[58]$} \\
STO/STO (KMC) & & 2.2 & 0.27 & {$[58]$} \\
SRO/STO (RHEED) & $1.0 \pm 0.2$ & & & {$[18]$} \\
SRO/STO (KMC) & & 0.75 & 0.6 & {$[18]$} \\
& & 1.3 & 0.3 & {$[213]$} \\
SRO/DSO (KMC) & & $0.9-1.0\left(E_{\mathrm{D}}{ }^{\mathrm{A}}\right)$ & 0.25 & {$[148]$} \\
& & $1.3\left(E_{\mathrm{D}}^{\mathrm{B}}\right)$ & 0.25 & \\
\hline
\end{tabular}

Tab. 5.1 Overview of reported $\mathrm{ABO}_{3}$ surface energetic values regarding surface diffusion. $E_{\mathrm{A}}$ is the activation energy, $E_{\mathrm{D}}$ is the diffusion energy barrier, $E_{\mathrm{D}}{ }^{\mathrm{A}}$ is the diffusion energy barrier on $\mathrm{AO}$ termination, $E_{\mathrm{D}}{ }^{\mathrm{B}}$ is the diffusion energy barrier on $\mathrm{BO}_{2}$ termination and $E_{\mathrm{N}}$ is the nearest neighbor energy. In literature, the surface energetic parameters are equal to $E_{\mathrm{A}}=1.0-2.2 \mathrm{eV}, E_{\mathrm{D}}=0.9-2.2 \mathrm{eV}$ and $E_{\mathrm{N}}=0.25-0.6 \mathrm{eV}$. The abbreviations STO, SRO, DSO are representing $\mathrm{SrTiO}_{3}, \mathrm{SrRuO}_{3}$ and $\mathrm{DyScO}_{3}$, respectively. Both, the experimental measured values using RHEED and the KMC simulation values are presented.

extracted from the linear fit in an Arrhenius plot ${ }^{2}$. The reported literature presents values of $E_{\mathrm{A}}=0.48-2.2 \mathrm{eV}$ for $\mathrm{SrTiO}_{3}$ (homoepitaxial growth) dependent on PLD conditions ${ }^{3}$ [58] and an $E_{\mathrm{A}}=1.0 \pm 0.2 \mathrm{eV}$ is found for $\mathrm{SrRuO}_{3}$ (grown on $\left.\mathrm{SrTiO}_{3}(001)\right)[18]$.

In the KMC model, diffusion of adatoms is simulated by calculating the hopping rates $k_{\text {hopping }}$ for all atoms, see Eq. 2.8. The hopping rate $k_{\text {hopping }}$ of a diffusive atom is proportional to $\sim \mathrm{e}^{\frac{-E_{\mathrm{D}}+n \cdot E_{\mathrm{N}}}{k_{\mathrm{B}} T}}$, where $E_{\mathrm{D}}$ is the diffusion energy barrier on the surface, $E_{\mathrm{N}}$ is the nearest neighbor energy and $n$ is the number of neighbor atomic bonds. $E_{\mathrm{D}}$ and $E_{\mathrm{N}}$ are necessary to calculate the $k_{\text {hopping }}$ of the diffusing

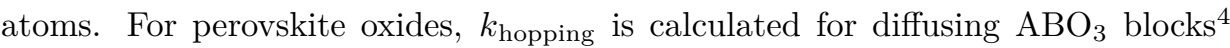
and the KMC simulations match with their growth experiments [17, 18, 148, 213]. The KMC model has been applied to predict $\mathrm{ABO}_{3}$ thin film growth, whereas it is hard to perceive as a free atomistic model for oxides as it is still unknown if single atoms or $\mathrm{ABO}_{3}$ units diffuse over the surface. Most likely, single atoms diffuse and form an $\mathrm{ABO}_{3}$ unit cell at the moment atoms attache to a step or island edge, resulting in an effective energy barrier (and which is observed in experiments).

In the atomistic model, known $E_{\mathrm{A}}$ (extracted from RHEED measurements) is not

\footnotetext{
${ }^{2}$ For both step-flow and $2 \mathrm{D}$ growth.

${ }^{3} \mathrm{An}$ increase of $E_{\mathrm{A}}$ from $0.48 \pm 0.05 \mathrm{eV}$ to $2.2 \pm 0.2 \mathrm{eV}$ has been observed for $\mathrm{SrTiO}_{3}$ increasing the PLD oxygen pressure from 3 to $20 \mathrm{~Pa}$.

${ }^{4}$ Diffusive $\mathrm{ABO}_{3}$ blocks instead of single atoms are simulated for simplification.
} 
sufficient as $E_{\mathrm{D}}$ and $E_{\mathrm{N}}$ contributions are still unknown. So far, iterative selection of $E_{\mathrm{D}}$ and $E_{\mathrm{N}}$ is done to match and elucidate growth experiments and KMC simulation. Tab. 5.1 shows the reported energetic values spread of $E_{\mathrm{D}}=0.75$ $2.2 \mathrm{eV}$ and $E_{\mathrm{N}}=0.25-0.6 \mathrm{eV}$ used in $\mathrm{ABO}_{3} \mathrm{KMC}$ simulations (in the case of the simulation of selective $\mathrm{SrRuO}_{3}$ growth, it has been shown that the EhrlichSchwoebel energy barrier $E_{\mathrm{ES}}$ has less influence on the results and is most of the time neglected [148]).

\subsubsection{Real-space observation of surface energetics}

In the semiconductor field, STM has been applied on Si and Ge surfaces to measure the kink energy $E_{\text {kink }}$ such that energetic parameters, such as $E_{\mathrm{N}}$ can be calculated from real-space observations [208]. The kink formation on vicinal surfaces is related to the amount of kink energy it costs to create a kink. The advantage of SPM's is that they can be used to resolve surface disruptions, such as kinks at step-edges with nanometer scale resolution, where RHEED averages the signal over a spatial area $>250 \mu \mathrm{m}$. STM can not be applied on most $\mathrm{ABO}_{3}$ substrates, because these materials are electrical insulators with large band gaps ${ }^{5}$. Therefore, the use of AFM is preferred to determine surface energetic parameters of $\mathrm{ABO}_{3}(001)_{(p) c}$ crystal surfaces, such as $\mathrm{E}_{\mathrm{N}}$, by measuring the kink-formation characteristics at $\mathrm{ABO}_{3}(001)$ step-edges ${ }^{6}$.

\footnotetext{
${ }^{5}$ For example, band gap of $\mathrm{DyScO}_{3}(110)_{o}$ is $5.7 \mathrm{eV}$.

${ }^{6}$ The kinks perpendicular to $\mathrm{ABO}_{3}(001)$ step-edges are thermally generated $\mathrm{ABO}_{3}$ unit cells existing of an $\mathrm{AO}$ and $\mathrm{BO}_{2}$ plane. To generate these kinks, the bond(s) have to be broken between two or more $\mathrm{ABO}_{3}$ unit cells.
} 


\subsection{Experiment}

A statistical analysis is performed to study kink formation at perovskite oxide step-edges. Fig. 5.2 a) shows an AFM image of a vicinal surface after applying the Canny method [219]. The lines in Fig. 5.2 a) represent the measured stepedges. In Fig. 5.2 b), a schematic representation of a perovskite oxide step-edge is shown. The continuous wavy line $w$ is representing the step meandering of the step-edge (white pixel lines in Fig. 5.2 a)) and $m$ is representing the mean line of the step-edge meandering. One way to extract the mean-square kink length is by measuring the deviation-deviation correlation function $G(r)$ of the step [208]:

$$
G(r)=<(h(r)-h(0))^{2}>=<k^{2}>\frac{r}{a_{0}}
$$

where $h(r)$ is the deviation measured in the direction perpendicular to the stepedge at position $r, h(0)=\langle h(r)>=0$ is the deviation measured in the direction perpendicular to the step-edge at position $0, r$ is the position along the high symmetry direction parallel to the step-edge, $a_{0}$ the unit cell length parallel to the step-edge and $\left\langle k^{2}>\right.$ is the mean-square kink length, see Fig. 5.2 b). From the mean-square kink length, the kink energy can be calculated as described in Eq. 5.3:

$$
<k^{2}>=\frac{\sum_{k=-\infty}^{\infty} k^{2} e^{\frac{-E_{\text {kink }}(k)}{k_{\mathrm{B}} T}}}{\sum_{k=-\infty}^{\infty} e^{\frac{-E_{\text {kink }}(k)}{k_{\mathrm{B}} T}}}
$$

where $k$ is the kink length, $k_{\mathrm{B}}$ the Boltzmann constant and $T$ the sample temperature in Kelvin. The kink energy is related to the nearest neighbor energy $E_{\mathrm{N}}$ according to:

$$
E_{\text {kink }}(k)=k \cdot E_{\mathrm{N}} / 2
$$

Using substitution the mean-square kink length $<k^{2}>$ and kink energy $E_{\text {kink }}$ relation can be simplified to:

$$
<k^{2}>=\frac{2 y}{(1-y)^{2}}
$$


a

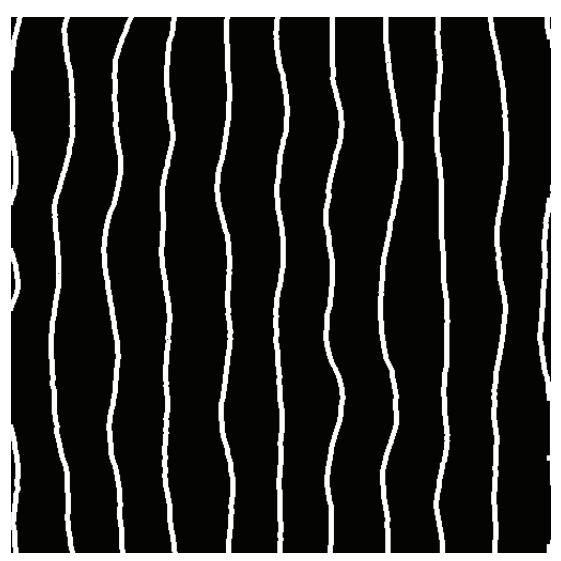

b

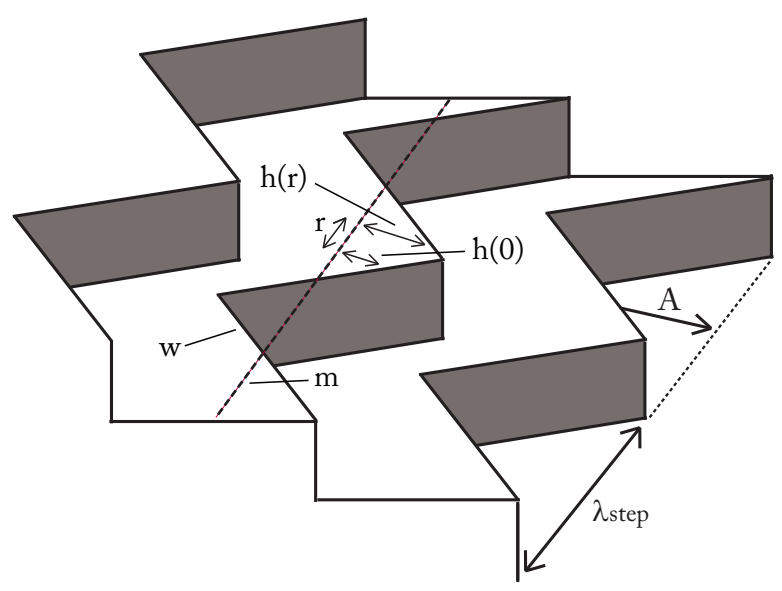

Fig. 5.2 Statistical analysis on a $512 \times 512$ pixels $^{2}$ ex situ TM-AFM image. a) Canny method applied to an AFM image of a $\mathrm{DyScO}_{3}(110)_{o}$ surface in thermodynamic equilibrium after annealing. The lines represent the step-edges. b) Schematic representation of a triangular step-edge undulation with amplitude A and wavelength $\lambda_{\text {step }}$. The wavy $w$ line represents a step-edge of the surface, whereas its mean line is called $m$. 
with,

$$
y=e^{\frac{-E_{\mathrm{kink}}}{k_{\mathrm{B}} T}}=e^{\frac{-E_{\mathrm{N}}}{2 k_{\mathrm{B}} T}}
$$

Eqs. 5.2 - 5.6 reveal that the kink energy $E_{\text {kink }}$ and therefore $E_{\mathrm{N}}$ can be extracted by measuring the slope $\left\langle k^{2}>\right.$ of the correlation function for small $r$. The linear dependence in the correlation function implies a random kink formation distribution.

A triangular step undulation model has been used to fit the correlation function of the perovskite oxide step waviness. This model is derived from a square-wave model expressing the total free energy $F$ for a square step [210]. It is used to elucidate strain relaxation behavior of $\mathrm{ABO}_{3}(001)_{(p) c}$ step-edges.

\subsection{Methods}

Standard commercial available $5 \times 5 \mathrm{~mm}^{2} \mathrm{LaAlO}_{3}(001)_{c}, \mathrm{SrTiO}_{3}(001)_{c}, \mathrm{DyScO}_{3}-$ $(110)_{o}, \mathrm{ZrO}_{2}: \mathrm{Y}(001)_{c}$ and $\mathrm{Al}_{2} \mathrm{O}_{3}(0001)_{h}$ (subscript $h$ denotes the hexagonal crystal structure) substrates were ordered from Crystec GmbH. Here, $\mathrm{LaAlO}_{3}(001)_{c}$, $\mathrm{SrTiO}_{3}(001)_{c}$ and $\mathrm{DyScO}_{3}(110)_{o}$ samples were taken as perovskite oxide model systems as they are the most commonly used substrates in thin film growth methods, chemically stable and are known to have a low defect density. In addition, these materials have a well-defined surface of alternating $\mathrm{AO}$ and $\mathrm{BO}_{2}$ planes, which is neutral in the case of $\mathrm{SrTiO}_{3}(001)_{c}$, polar for $\mathrm{DyScO}_{3}(110)_{o}$ and polar for $\mathrm{LaAlO}_{3}(001)_{c}$ (with a phase transition below $830 \mathrm{~K}$ ) [220, 221]. Furthermore, $\mathrm{LaAlO}_{3}(001)_{c}, \mathrm{SrTiO}_{3}(001)_{c}$ and $\mathrm{DyScO}_{3}(110)_{o}$ have a wide range of pseudo-cubic lattice constants in the perovskite oxide spectrum of $a_{0}=0.3821 \mathrm{~nm}, 0.3905 \mathrm{~nm}$ and $0.395 \mathrm{~nm}$, respectively [42]. Other oxides, such as $\mathrm{ZrO}_{2}: \mathrm{Y}(001)_{c}$ (fluorite) and $\mathrm{Al}_{2} \mathrm{O}_{3}(0001)_{h}$ (corundum) were selected as these materials have a different crystal structure and local oxygen coordination at the surface as compared to the perovskite oxides mentioned before [67].

Prior to the annealing procedure, all samples were ultrasonically rinsed in acetone and ethanol both for $10 \mathrm{~min}$. After rinsing, substrate treatments were applied to achieve single terminated substrates in order to ensure a $\mathrm{BO}_{2}$ terminated plane at the surface $[19,20]$. Then, the samples were annealed in a tube furnace using an $\mathrm{O}_{2}$ flow of $150 \mathrm{l} / \mathrm{h}$ at a temperature of $1000{ }^{\circ} \mathrm{C}$. The annealing time and temperature were varied in order to find at which temperature the surface equilibrates. All AFM images were taken ex situ in tapping mode (TM) using a Bruker ICON AFM 
and a Bruker TESPA doped Si cantilever $f_{0}=320 \mathrm{kHz}$. Step-edge analysis was performed on the AFM images by first applying a Gaussian filter in order to remove any noise. Subsequently, leveling of the height data was done by plane fitting the topography. Step-edges were detected by applying the Canny method [219]. The surface steps were aligned perpendicular to the fast scan direction to prevent tip artifacts in the AFM image.

\subsection{Experimental results \& discussion}

The experimental results are shown and discussed in this section. First, experiments are done to confirm that the studied surfaces are in thermodynamic equilibrium. Thereafter, the terrace width distribution and the correlation functions of perovskite oxide $(001)_{(p) c}$ are shown, which are used to extract energetic parameters, such as the nearest neighbor energy. A model is proposed to describe the observed step-edge undulation. Finally, a terrace width dependence of the correlation function is presented together with the correlation function of oxides with a different local oxygen coordination at the surface.

\subsubsection{Thermodynamic equilibrium}

Figs. 5.3 (a-d) show four AFM images of a $\mathrm{DyScO}_{3}(110)_{o}$ surface a) as-received and after b) $30 \mathrm{~min}$, c) $4 \mathrm{~h}$, and d) $12 \mathrm{~h}$ of annealing, respectively. The polished as-received surface exhibits disordered step-edges, without a well-defined step distribution. After 30 min annealing, the steps become visible, but are still slightly disordered. Some terraces display one layer deep vacancy islands, which are located in the vicinity of the step-edges. Prolonged annealing up to $4 \mathrm{~h}$ results in meandering of the surface steps and a well-defined and narrow terrace width distribution. No vacancy islands are present in the terraces after $4 \mathrm{~h}$ annealing. Continuing annealing to $12 \mathrm{~h}$ reveals no change of the surface or step-edge correlation function analysis.

Our assumption that the $\mathrm{DyScO}_{3}(110)_{o}$ surface is in equilibrium after annealing more than $4 \mathrm{~h}$ at $1000{ }^{\circ} \mathrm{C}$ is based on our AFM observations. The length scale at which equilibrium is reached scales as $t^{\alpha}$, where $t$ is the time and $\alpha$ an exponent that depends on the exact mass transport mechanism [222, 223, 224]. An exponent $\alpha=\frac{1}{2}$ describes mass exchange between the step-edge and adatoms on the terrace, whereas $\alpha=\frac{1}{4}$ describes mass transport along step-edges [225]. The large wavelength along the meandering step-edge suggests that a non-equilibrium step instability is unlikely $[226,227]$. Furthermore, $\mathrm{SrTiO}_{3}(001)_{c}$ samples annealed 


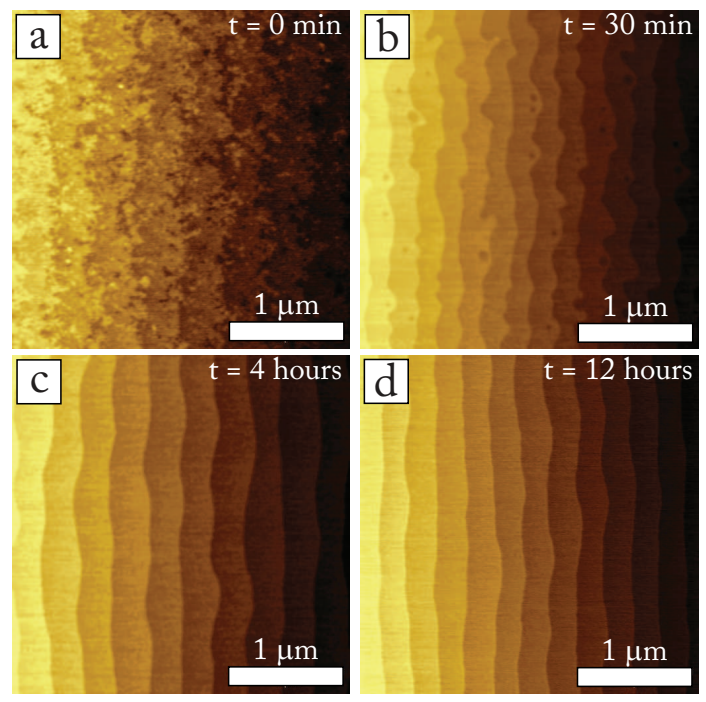

Fig. 5.3 Ex situ TM-AFM images of $\mathrm{DyScO}_{3}(110)_{o}$ surface at room temperature of a) an as-received surface, b) surface after 30 min annealing at $1000{ }^{\circ} \mathrm{C}$, c) surface after $4 \mathrm{~h}$ annealing at $1000{ }^{\circ} \mathrm{C}$ and d) surface after $12 \mathrm{~h}$ annealing at $1000{ }^{\circ} \mathrm{C}$, respectively. Note that these samples were cut from the same crystal.

for $16 \mathrm{~h}$ at $1000{ }^{\circ} \mathrm{C}$ show a similar correlation function and surface morphology (measured with AFM) compared to $\mathrm{SrTiO}_{3}(001)_{c}$ samples annealed for $4 \mathrm{~h}$.

\subsubsection{Terrace width distribution}

The terrace width distribution $\mathrm{P}(l)$ vs. the terrace width $l$ is extracted for the $4 \mathrm{~h}$ annealed $\mathrm{DyScO}_{3}(110)_{o}$ surface and shown in Fig. 5.4. Neglecting the weak shoulders and the slight asymmetry in the distribution, the data has been fitted with a Gaussian. An average terrace width $<L>$ of $760 \mathrm{a}_{0} \equiv 300 \mathrm{~nm}$ is found for this particular surface. The width $\sigma$ of this distribution is $86 a_{0} \equiv 34 \mathrm{~nm}$, which is relatively small and indicative for step-step interactions. Typically, $\langle L\rangle$ and $\sigma$ are measured in order to calculate the strength of the step-step interactions. This very narrow distribution of $\mathrm{DyScO}_{3}(110)_{o}$ compared to Si can be explained by the coherent behavior of the $\mathrm{DyScO}_{3}(110)_{o}$ step-edges [208]. The right shoulder in the distribution is most probably present due to step-step repulsion. 


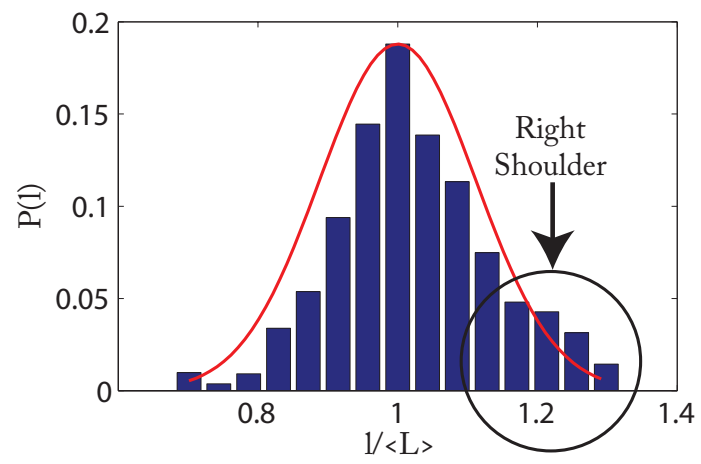

Fig. 5.4 Normalized terrace width distribution $\mathrm{P}(l)$ vs. terrace width $l$ of the $\mathrm{DyScO}_{3}(110)_{o}$ surface annealed for $4 \mathrm{~h}$ at $1000{ }^{\circ} \mathrm{C}$. An average terrace width $\langle L\rangle=760 \mathrm{a}_{0}$ is found for the $4 \mathrm{~h}$ annealed $\mathrm{DyScO}_{3}(110)_{o}$ surface. The narrow width $\sigma=86 a_{0}$ and asymmetry of the distribution are indicative for entropic and energetic step-step interactions.

\subsubsection{Correlation functions perovskite oxides $(001)_{(p) c}$}

In Fig. 5.5 a), the average correlation functions are shown for $\operatorname{SrTiO}_{3}(001)_{c}(\mathrm{AFM}$ image - Fig. 5.7 d)) and $\mathrm{DyScO}_{3}(110)_{o}$ (AFM image - Fig. 5.3 c)) step-edges with in the inset the correlation function of a single step-edge together with a zoom-in of the correlation functions for $r$ ranging from $0-200 a_{0}$ in Fig. 5.5 b). At large length scales, see Fig. 5.5 a), the correlation functions of both systems show a clear correlation with an average periodicity of $\lambda_{\text {step }} \approx 2100 a_{0} \equiv 830 \mathrm{~nm}$, which slightly varies from step to step. Multiple minima and maxima are present demonstrating the strong correlation along the step-edges. At short length scales, see Fig. $5.5 \mathrm{~b}$ ), the correlation function starts to deviate from the linear fit $\left(G(r) \sim r,\left\langle k^{2}\right\rangle=\right.$ $\left.2.1 \pm 0.1 a_{0}^{2}\right)$ around $r>60 a_{0}$ to a quadratic relation $\left(G(r) \sim r^{2}\right)$. The ratio between minima and maxima and amplitude A (see Fig. 5.2 b)) slightly varies from step to step, but the periodicity remains constant for both material systems. The correlation along the $\mathrm{DyScO}_{3}(110)_{o}$ surface steps depends on the average terrace width $\left\langle L>\right.$. When the $\mathrm{DyScO}_{3}(110)_{o}$ average terrace width is smaller than $<L>$ $<170 \mathrm{~nm}$, the correlation function becomes less pronounced and the mean-square kink length decreases. 
a

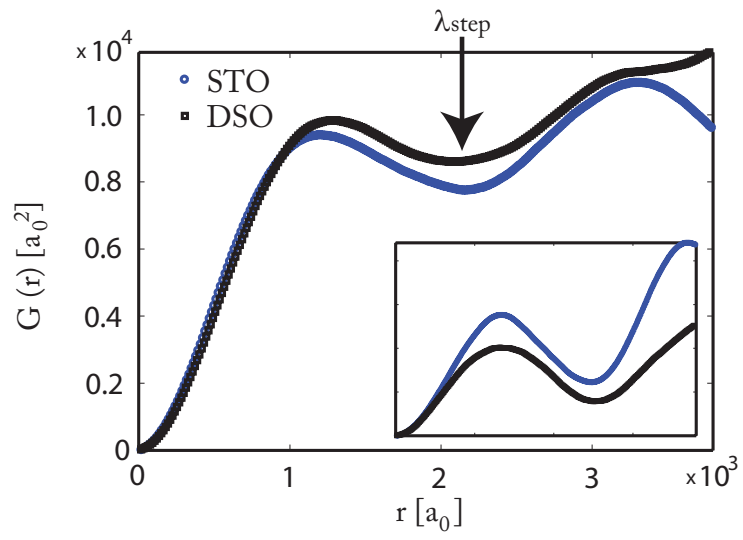

$\mathrm{b}$

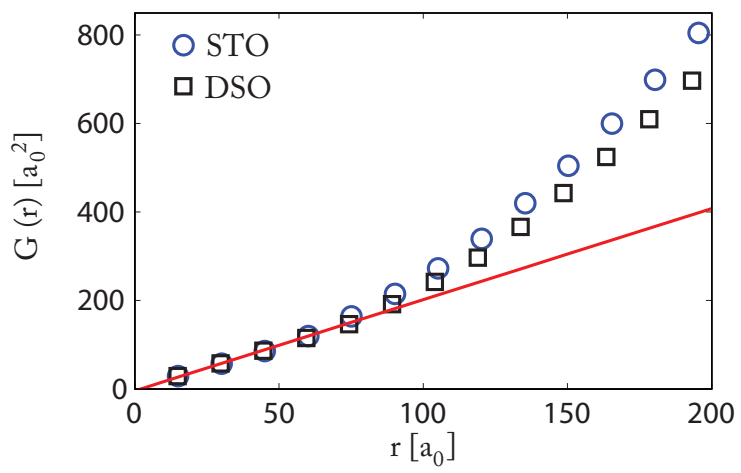

Fig. 5.5 Average correlation functions a) of $\mathrm{SrTiO}_{3}(001)$ (STO) and $\mathrm{DyScO}_{3}(110)$ (DSO) surface step-edges with in the inset a correlation function of a single step-edge. The correlation functions show an average periodicity of $\lambda_{\text {step }} \approx 2100 a_{0} \approx 830 \mathrm{~nm}$ for both surfaces, which slightly varies from step-edge to step-edge. In b) a zoom-in $r=[0$ 200] $a_{0}$ of the correlation functions is shown. The average terrace widths of the samples investigated are in between $\langle L\rangle=250-300 \mathrm{~nm}$.

\subsubsection{Model}

The triangular step undulation is indicative for a strong energy relaxation mechanism along the step. There is a competition between two energy terms in minimizing the surface free energy, the energy cost to create additional step length and the energy reduction by strain relaxation due to the triangular undulations along the step-edge.

The total free energy of a simple square-wave like step with periodicity $\lambda_{\text {step }}$ can 
be described as [210]:

$$
F=\gamma+\frac{2 F_{\text {step }}}{\lambda_{\text {step }}}-\frac{(1-v)(\Delta \sigma)^{2}}{\pi \mu \lambda_{\text {step }}} \ln \left(\frac{\lambda_{\text {step }}}{2 \pi a} \cdot \sin (\pi p)\right)
$$

where $\gamma$ is the free energy per unit area (in perovskite oxides, the free energy per unit area for a step-up and step-down domain are equal), $F_{\text {step }}$ is the step free energy per unit length, $v$ is the Poisson ratio $(v=0.24$ for single crystal $\mathrm{SrTiO}_{3}$ ) [228], $\mu$ the bulk modulus (183 $\mathrm{GPa}$ for $\mathrm{SrTiO}_{3}$ ) [229] and $\Delta \sigma$ the force monopole at the step-edge. Perovskite oxide step-edge undulations have a more triangular wave-like behavior. The total free energy per unit area of a triangular step-edge can be written as:

$$
\left.F=\frac{1}{A}\left[\int_{h=0}^{A} \gamma d h+\int_{h=0}^{A} \frac{2 F_{\text {step }}}{\lambda_{\text {step }}} d h-\int_{h=0}^{A} \frac{2 C}{\lambda_{\text {step }}} \ln \left(\frac{\lambda_{\text {step }}}{2 \pi a}\right) d h-\int_{h=0}^{A} \frac{2 C}{\lambda_{\text {step }}} \sin (\pi p)\right) d h\right]
$$

where,

$$
C=\frac{(1-v)(\Delta \sigma)^{2}}{2 \pi \mu}
$$

and,

$$
p=\frac{A-h}{A}
$$

Eq. 5.8 can be rewritten as:

$$
F=\gamma+\frac{2 F_{\text {step }}}{\lambda_{\text {step }}}-\frac{2 C}{\lambda_{\text {step }}} \ln \left(\frac{\lambda_{\text {step }}}{2 \pi a}\right)+\frac{2 C}{\lambda_{\text {step }} \pi} \int_{\pi}^{0} \ln [\sin (x)] d x
$$

The integral of Eq. 5.11 can be solved by using trigonometric identities and integration by substitution: 


$$
\begin{aligned}
\int_{\pi}^{0} \ln [\sin (x)] d x & =\int_{\pi}^{0} \ln \left[2 \sin \left(\frac{x}{2}\right) \cos \left(\frac{x}{2}\right)\right] d x \\
& =\int_{\pi}^{0} \ln (2) d x+\int_{\pi}^{0}\left[\sin \left(\frac{x}{2}\right)\right] d x+\int_{\pi}^{0}\left[\cos \left(\frac{x}{2}\right)\right] d x \\
& =-\pi \ln (2)+2 \int_{\frac{\pi}{2}}^{0}[\sin (y)] d y+\int_{\pi}^{0}\left[\sin \left(\frac{x+\pi}{2}\right)\right] d x \\
& =-\pi \ln (2)+2 \int_{\frac{\pi}{2}}^{0}[\sin (y)] d y+2 \int_{\pi}^{\frac{\pi}{2}}[\sin (y)] d x \\
& =-\pi \ln (2)+2 \int_{\pi}^{0}[\sin (y)] d y
\end{aligned}
$$

Since $\int_{\pi}^{0} \ln [\sin (x)] d x=\pi \ln (2)$ see Eq. 5.12, the total free energy per unit area $F$ of a triangular step-edge is equal to:

$$
F=\gamma+\frac{2 F_{\text {step }}}{\lambda_{\text {step }}}-\frac{2 C}{\lambda_{\text {step }}} \ln \left(\frac{\lambda_{\text {step }}}{2 \pi a}\right)+\frac{2 C \ln (2)}{\lambda_{\text {step }}}
$$

The critical periodicity $\lambda_{\text {stepminF }}$ follows from the minimum free energy per unit area by calculating $\frac{d F}{d \lambda_{\text {step }}}=0$ as:

$$
\lambda_{\text {stepminF }}=2 \pi a e^{\left(\frac{F_{\text {step }}}{C}+1+\ln (2)\right)}
$$

Besides the $\ln (2)$ term in the exponent, Eq. 5.14 is identical to the relation derived by Alerhand et al. [230]. From the correlation function, the critical periodicity $\lambda_{\text {stepc }}$ is found to be equal to $2100 a_{0} \approx 830 \mathrm{~nm}$ which results in a ratio of $\frac{F_{\text {step }}}{C}=$ $\frac{E_{\text {kink }}}{C}=4.13$. From Fig. 5.5, a $<k^{2}>=2.1 \pm 0.1 a_{0}{ }^{2}$ is extracted assuming random kink formation on short length scale, which results in $E_{\text {kink }}=0.10 \mathrm{eV} / a_{0}$ using Eqs. 5.5 - 5.6. The nearest neighbor energy $E_{\mathrm{N}}$ can be determined using $E_{\mathrm{N}}=2$ $E_{\text {kink }}=0.21 \pm 0.01 \mathrm{eV}$ for $\mathrm{SrTiO}_{3}(001)_{c}$ and $\mathrm{DyScO}_{3}(110)_{o}$ as a (pseudo-)cubic system [212]. Furthermore, the strain relaxation energy constant $\mathrm{C}$ is found to be equal to $24 \mathrm{meV} / a_{0}$. The nearest neighbor energy is close to $E_{\mathrm{N}}=0.25-0.6 \mathrm{eV}$, a value commonly used in KMC simulations to simulate thin film growth [213]. The 
proposed model explains the quasi 1D character of a perovskite oxide step-edge for terrace widths larger than $170 \mathrm{~nm}$. Smaller terrace widths result in a more 2D character caused by the step-step interaction, which requires a more sophisticated model.

\subsubsection{Terrace width dependence:}

The small terrace width distribution of $\mathrm{DyScO}_{3}(110)_{o}$ is indicative of step-step interactions. In the case that step-step interactions are present, a change in the stepedge morphology, correlation function and extracted energetic values should be found for smaller terrace widths. From Fig. 5.6, it can be seen that $\mathrm{DyScO}_{3}(110)_{o}$ vicinal surfaces with a small average terrace width below $\langle L\rangle=170 \mathrm{~nm}$ results in a change in step-edge morphology, correlation function and a different extracted nearest neighbor energy $E_{\mathrm{N}}$.

Here, a mean-square kink length is found of $<k^{2}>=2.1 \pm 0.1 a_{0}^{2}$ for $\operatorname{DyScO}_{3}(110)_{o}$ having average terrace widths of $\langle L\rangle=170 \mathrm{~nm}$ and $300 \mathrm{~nm}$. For both, a similar correlation function behavior is found on small length scales $\left(0-100 a_{0}\right)$, but on large length scales $\left(1000-3000 a_{0}\right)$ the correlation has less pronounced minima and maxima for the $\mathrm{DyScO}_{3}(110)_{o}$ surface having $\langle L\rangle=170 \mathrm{~nm}$. A smaller mean-square kink length of $\left\langle k^{2}\right\rangle=1.6 \pm 0.1 a_{0}^{2}$ is found for the $\operatorname{DyScO}_{3}(110)_{o}$ surface with an average terrace width of $92 \mathrm{~nm}$.

The mean-square kink length decreases with larger step-step repulsion as the entropy of steps decreases for small step-step separations. Typically, the influence of step-step interaction potential on a meandering step increases with $1 / L^{2}$ [208]. The decrease of the mean-square kink length $\left\langle k^{2}\right\rangle=2.1 \pm 0.1 a_{0}^{2}$ to $\left\langle k^{2}\right\rangle=1.6$ $\pm 0.1 a_{0}^{2}$ results in a change of the found nearest neighbor energy of $E_{\mathrm{N}}=0.21$ $\pm 0.01 \mathrm{eV}$ to $0.23 \pm 0.01 \mathrm{eV}$. $\mathrm{DyScO}_{3}(110)_{o}$ surfaces with smaller terrace widths have a more $2 \mathrm{D}$ character caused by the step-step interaction, which requires a more sophisticated model. Both, the entropic step-step interaction [231] and step interaction energy models [230] used to describe the interaction of Si step-edges are not valid for $\mathrm{DyScO}_{3}(110)_{o}$ step-edges. The models assume a meandering $\mathrm{Si}$ step-edge catched between two straight step-edges, while the triangular undulation of step-edges on a $\mathrm{DyScO}_{3}(110)_{o}$ surface behaves coherent. 

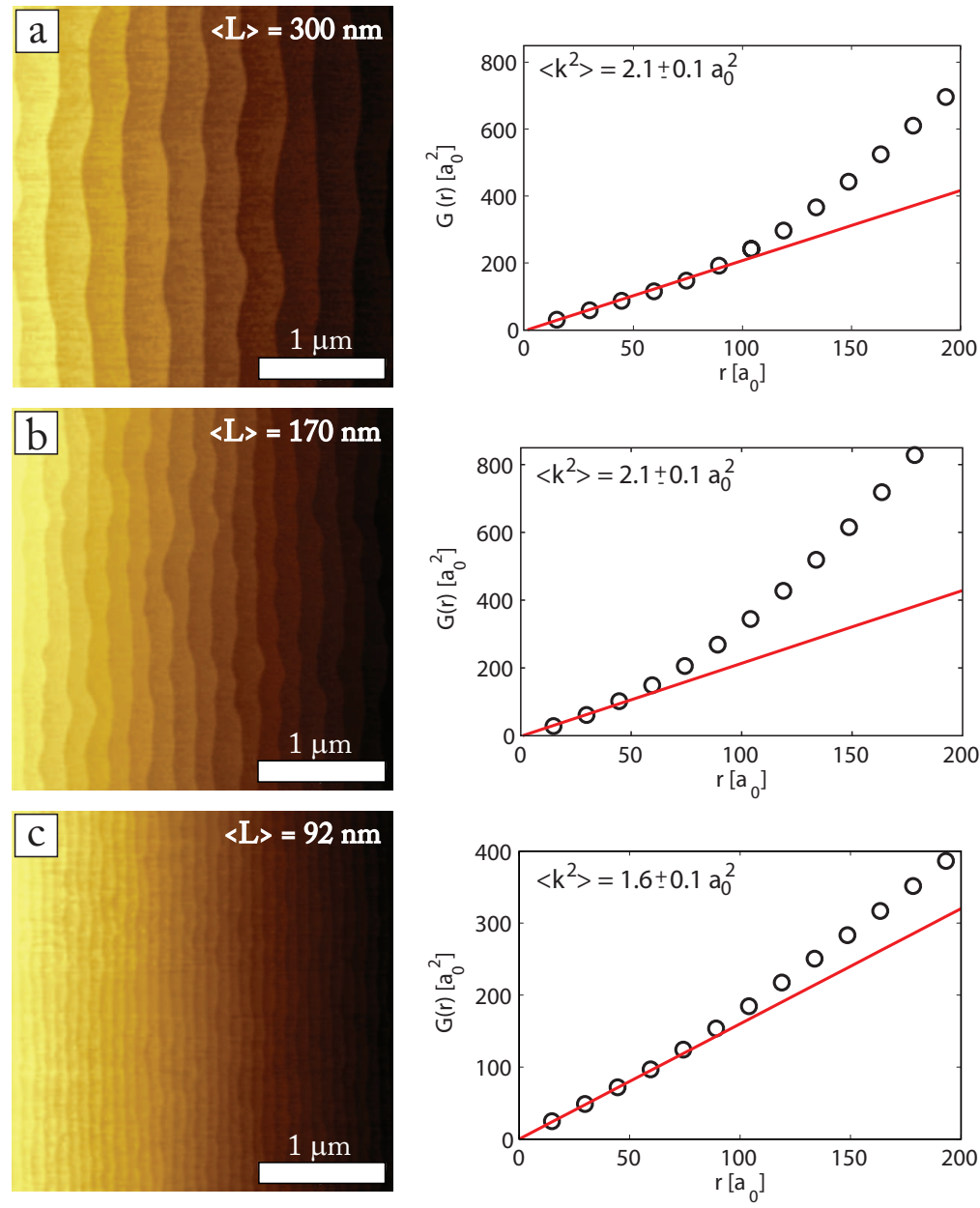

Fig. 5.6 Ex situ TM-AFM images of annealed $\mathrm{DyScO}_{3}(110)_{o}$ exhibiting different average terrace widths $\langle\mathrm{L}\rangle$ in the left panel. In the right panel the accompanying average correlation functions. The mean-square kink length $<k^{2}>$ decreases from $2.1 \pm 0.1 a_{0}{ }^{2}$ to $1.6 \pm 0.1 a_{0}^{2}$ below $\langle L\rangle=170 \mathrm{~nm}$. 


\subsubsection{Oxides with different oxygen coordination}

Different oxide materials were selected to compare the energetics of the perovskite oxide $\mathrm{LaAlO}_{3}(001)_{c}$ with different local oxygen coordinated oxides, such as $\mathrm{ZrO}_{2}: \mathrm{Y}(001)_{c}$ and $\mathrm{Al}_{2} \mathrm{O}_{3}(0001)_{h}$. Note that the average terrace widths $\langle L>$ in the same range $(80-101 \mathrm{~nm})$ were selected on purpose as the found mean-square kink length $\left\langle k^{2}>\right.$ depends on the terrace width $\langle L>$. Fig. 5.7 shows the AFM images of the different oxide materials used to extract the correlation functions.

From the AFM images in Fig. 5.7, it can be seen that the step-edge morphology is different for $\mathrm{LaAlO}_{3}(001)_{c}(<L>=89 \mathrm{~nm}), \mathrm{ZrO}_{2}: \mathrm{Y}(001)_{c}(<L>=101 \mathrm{~nm})$ and $\mathrm{Al}_{2} \mathrm{O}_{3}(0001)_{h}(<L>=80 \mathrm{~nm})$. The stepped morphology of the $\mathrm{LaAlO}_{3}(001)_{c}$ surface is similar to $\mathrm{DyScO}_{3}(110)_{o}$ with almost the same terrace width. The $\mathrm{LaAlO}_{3}(001)_{c}$ surface contains single unit cell steps. In contrary, the step-edge height of $\mathrm{ZrO}_{2}: \mathrm{Y}(001)_{c}$ and $\mathrm{Al}_{2} \mathrm{O}_{3}(0001)_{h}$ consists of a single atomic layer, while for these type of oxides the unit cell exist of two and four atomic layers, respectively. $\mathrm{ZrO}_{2}: \mathrm{Y}(001)_{c}$ exhibits a less coherent behavior in the stepped morphology compared to $\mathrm{LaAlO}_{3}(001)_{c}$. XPS measurements (performed in the same manner as described in Ch. 6) show no significant Yttrium segregation at the sur-

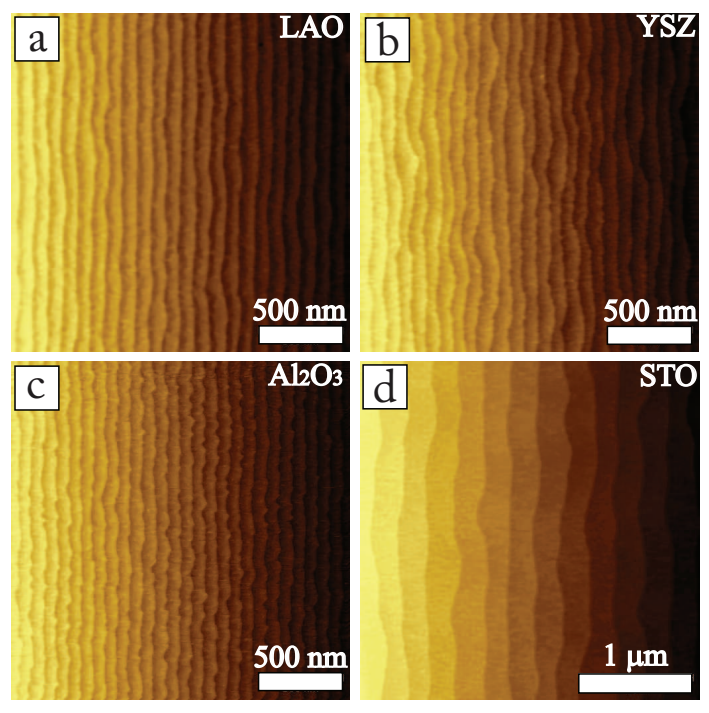

Fig. 5.7 Ex situ TM-AFM images of an annealed a) $\mathrm{LaAlO}_{3}(001)_{c}$ (LAO) perovskite surface, b) $\mathrm{ZrO}_{2}: \mathrm{Y}(001)_{c}$ (YSZ) fluorite surface, c) $\mathrm{Al}_{2} \mathrm{O}_{3}(0001)_{h}$ (sapphire) hexagonal surface. The average terrace width of LAO, YSZ, sapphire is $\langle L\rangle=89 \mathrm{~nm},\langle L\rangle=101$ $\mathrm{nm}$ and $\langle L\rangle=80 \mathrm{~nm}$, respectively. d) TM-AFM image of the annealed $\mathrm{SrTiO}_{3}(001)_{c}$ surface $(<L>=250 \mathrm{~nm})$ used for analysis. 


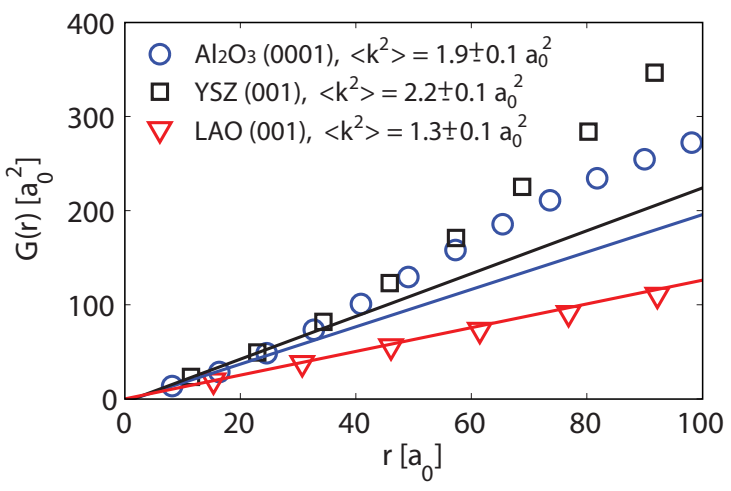

Fig. 5.8 Average correlation functions of an annealed a) $\mathrm{LaAlO}_{3}(001)_{c}(\mathrm{LAO})$ perovskite surface, b) $\mathrm{ZrO}_{2}: \mathrm{Y}(001)_{c}$ (YSZ) fluorite surface, c) $\mathrm{Al}_{2} \mathrm{O}_{3}(0001)_{h}$ (sapphire) corundum surface. The found $E_{\mathrm{N}}$ values of LAO $(<L>=89 \mathrm{~nm})$, YSZ $(<L>=101 \mathrm{~nm})$, sapphire $(<L>=80 \mathrm{~nm})$ are $0.26 \pm 0.01 \mathrm{eV}, 0.20 \pm 0.01 \mathrm{eV}$ and $0.09 \pm 0.01 \mathrm{eV}$, respectively.

face [232]. The $\mathrm{Al}_{2} \mathrm{O}_{3}(0001)_{h}$ stepped surface morphology is rough compared to $\mathrm{LaAlO}_{3}(001)_{c}$, showing no signature of a triangular undulation and the steps are less coherent. Furthermore, an AFM image is depicted of the $\mathrm{SrTiO}_{3}(001)_{c}$ surface as comparison to $\mathrm{DyScO}_{3}(110)_{o}$ used for extraction of the correlation function. From the stepped morphology of $\mathrm{SrTiO}_{3}(001)_{c}$, it can be seen that this vicinal surface is similar to the $4 \mathrm{~h}$ annealed $\mathrm{DyScO}_{3}(110)_{o}$ vicinal surface.

For the $\mathrm{LaAlO}_{3}(001)_{c}$ surface having $\left\langle L>=89 \mathrm{~nm}\right.$, a slightly lower $<k^{2}>=1.3$ $\pm 0.1 a_{0}^{2}$ was found compared to $<k^{2}>=1.6 \pm 0.1 a_{0}^{2}$ found for $\operatorname{DyScO}_{3}(110)_{o}$ having $\left\langle L>=92 \mathrm{~nm}\right.$. The correlation function of $\mathrm{LaAlO}_{3}(001)_{c}$ is plotted in Fig. 5.8 together with other types of oxides having different local oxygen coordination, such as $\mathrm{ZrO}_{2}: \mathrm{Y}(001)_{c}$ and $\mathrm{Al}_{2} \mathrm{O}_{3}(0001)_{h}$. An $E_{\mathrm{N}}$ of $0.26 \pm 0.01 \mathrm{eV}, 0.20$ $\pm 0.01 \mathrm{eV}$ and $0.09 \pm 0.01 \mathrm{eV}$ is found for small terrace widths $(80-100 \mathrm{~nm})$ on $\mathrm{LaAlO}_{3}(001)_{c}, \mathrm{ZrO}_{2}: \mathrm{Y}(001)_{c}$ and $\mathrm{Al}_{2} \mathrm{O}_{3}(0001)_{h}$, respectively. For $\mathrm{Al}_{2} \mathrm{O}_{3}(0001)_{h}$, a hexagonal unit cell was used in order to calculate $E_{\mathrm{N}}$ for its triangular lattice [211].

From the comparable step meandering along the step-edges of perovskite oxides and contrast to other oxide types, it is most likely that the surface energetics depends on the local oxygen coordination. According to this remark, the surface energetics should change with different alternating planes, for example in the case of $\mathrm{SrTiO}_{3}(001)_{c} / \mathrm{AO}-\mathrm{BO}_{2}, \mathrm{SrTiO}_{3}(110)_{t} / \mathrm{ABO}-\mathrm{O}_{2}$ (subscript $t$ denotes the tetragonal crystal structure) and $\mathrm{SrTiO}_{3}(111)_{h} / \mathrm{AO}_{3}-\mathrm{B}$, which is supported by DFT calculations and difference in required annealing times [233]. The importance of 
the oxygen coordination is also supported by the specific terminating plane at the surface, as it has been shown that nanostructures can be grown selective on mixed terminated substrates [217, 234]. Furthermore, the oxygen coordination changes with a termination switch and therefore an expected change in surface energetics might explain the difference in interface properties of oxide heterostructures [235].

\subsection{Conclusions \& outlook}

In conclusion, perovskite oxide vicinal surfaces are analyzed after annealing $\mathrm{LaAlO}_{3}$ $(001)_{c}, \mathrm{SrTiO}_{3}(001)_{c}$ and $\mathrm{DyScO}_{3}(110)_{o}$ substrates. Using the deviation-deviation correlation function, triangular step-edge undulations are found with an average periodicity of $\lambda_{\text {step }} \approx 2100 a_{0}$. This step undulation is caused by strain relaxation along the step direction. The ratio between step formation energy and elastic interaction is calculated using the Marchenko-Alerhand model applied for a triangular perovskite oxide step with average terrace widths of 250 - $300 \mathrm{~nm}$. A kink formation energy $E_{\text {kink }}=0.10 \mathrm{eV}$ is found for the perovskite oxides $\operatorname{SrTiO}_{3}(001)_{c}$ and $\mathrm{DyScO}_{3}(110)_{o}$. Assuming a square lattice with isotropic nearest neighbor interactions, a nearest neighbor energy of $E_{\mathrm{N}}=0.21 \pm 0.01 \mathrm{eV}$ is determined. This nearest neighbor interaction energy is close to $E_{\mathrm{N}}=0.25-0.6 \mathrm{eV}$ commonly used in KMC simulations to simulate perovskite oxide thin film growth. The experiments reveal that the surface energetics of perovskite oxide surfaces are mainly governed by the local oxygen coordination. It might result in a similar behavior of the oxygen exchange and defect formation in perovskite oxide heterostructures at surfaces and interfaces. Finally, it needs to be emphasized that a change in perovskite oxide alternating stacking or terminating plane might influence the surface energetics due to the difference in local oxygen coordination at the surface.

The following experiments are suggested to further validate the assumption that the surface energetics of perovskite oxides are governed by the oxygen coordination at the surface:

- Studying of different $\mathrm{ABO}_{3}$ crystal orientations, such as $(001)_{c},(110)_{t}$ and $(111)_{h}$ under the same conditions. It is expected that the stepped morphology and the correlation function will change with crystal orientation as the oxygen coordination changes.

- Annealing experiments at different oxygen pressures to create oxygen vacancies. Oxygen deficient surfaces might for example lead to a change in nearest neighbor energy. 



\title{
Chapter 6
}

\section{Sticking of volatile species in perovskite oxides}

\begin{abstract}
Stoichiometric transfer of species from target to film during pulsed laser deposition (PLD) is often a prerequisite for optimization of perovskite oxide structural and physical properties. However, when diffusive atoms on a surface are prone to re-evaporate, offstoichiometric films can be obtained. These volatile species have a lower probability to stick at a step-edge, before the species start to re-evaporate from the surface. One way to increase the sticking probability of volatile species is to increase the step density and therefore shortening of the average distance between adatoms and step-edges. In this chapter, the influence of temperature modulation pulsed laser deposition (TM-PLD) on the growth (step density) and sticking of volatile species is investigated. Both kinetic Monte Carlo (KMC) simulations and experiments of $\mathrm{SrTiO}_{3}$ homoepitaxial growth show that the step density modulation during layer-by-layer growth can be increased using TMPLD compared to PLD at constant temperature. Furthermore, this method has been applied during $\mathrm{PbTiO}_{3}$ growth, where $\mathrm{Pb}$ is the volatile element. In the temperature range where $\mathrm{Pb}$ starts to re-evaporate, an increase in $\mathrm{Pb}$ :Ti ratio is observed for TM$\mathrm{PLD} \mathrm{PbTiO}_{3}$ films compared to $\mathrm{PbTiO}_{3}$ films deposited only at the higher temperature during TM-PLD.
\end{abstract}

W.A. Wessels, G. Koster and G. Rijnders, "Sticking of volatile species in perovskite oxides", in preparation. 


\subsection{Introduction}

Pulsed laser deposition (PLD) is a relative simple and versatile technique to deposit complex thin film materials, such as perovskite oxides [3]. One key feature of PLD is the stoichiometric transfer of species from target to thin film material $[7,127]$. Therefore, most thin films can be fabricated containing the stoichiometry of the target. However, for some perovskite oxide thin films stoichiometry is hard to achieve as these materials contain volatile elements, e.g. $\mathrm{Pb}, \mathrm{Na}$ and $\mathrm{Bi}$ [127]. The volatile elements might desorb from the surface after absorption and diffusion due to effects, such as re-evaporation [127] and re-sputtering [236]. When these effects do not take place, atoms diffuse until they become attached to a step-edge, which is called "sticking" of adatoms. Here, the sticking coefficient is defined as the ratio between the number of incorporated elements in the film (elements that "stick") and the number of arriving elements.

Off-stoichiometric thin films are often unwanted as it strongly influences the structural and physical properties. For example, the transport properties have been negatively affected by cation off-stoichiometry in $\mathrm{Pt} / \mathrm{Nb}-\mathrm{SrTiO}_{3}$ junctions [237]. Furthermore, high leakage currents in $\mathrm{BiFeO}_{3} / \mathrm{PbTiO}_{3}$ are ascribed to volatilization of $\mathrm{Bi} / \mathrm{Pb}$ [238]. The lattice parameter and growth mode of homoepitaxial $\mathrm{SrTiO}_{3}$ films depends strongly on the oxygen content in the film [126]. On the other hand, Zalutayev et al. report that cation off-stoichiometry can be beneficial for high p-type conductivity and transparency in $\mathrm{Co}_{2} \mathrm{ZnO}_{4}$ and $\mathrm{Co}_{2} \mathrm{NiO}_{4}$ thin films [239]. These studies show that control of stoichiometry leads to control of structural and physical properties.

Previously, several manners have been used in order to achieve stoichiometric films of materials containing volatile species. Frequently, non-stoichiometric targets are used to compensate for the loss of the volatile species [127]. The amount of excess of volatile species put in a target was based on how much of the volatile species re-evaporates. An other method, which was proposed to obtain stoichiometric films containing volatile elements, was the use of segmented evaporation targets [240]. Using segmented targets of $\mathrm{KNbO}_{3}, \mathrm{KTO}_{3}$ and cold-compressed $\mathrm{KN}_{3}$ (extra potassium source), Christen et al. succeeded to grow stoichiometric epitaxial $\mathrm{KNbO}_{3}$ films and $\mathrm{KNbO}_{3} / \mathrm{KTO}_{3}$ superlattices [241]. Recently, pulsed laser interval deposition (PLID) was applied in order to increase the sticking of Bi during deposition of $\mathrm{YBiO}_{3}$ and $\mathrm{BaBiO}_{3}$ films at a growth effective temperature, where Bi becomes volatile [242].

PLD parameters, such as the deposition flux and deposition temperature are expected to have an influence on both the nucleation density and diffusivity of 
adatoms. An increase of deposition flux results in an increase of nucleation density and on its turn increases the probability for adatoms to stick at a step-edge before re-evaporation [44]. Using deposition flux modulation, first the step density is kept high, whereafter adatoms have longer time to diffuse before new material is ablated. This deposition flux modulation method has been used to impose layerby-layer growth of superconductors, whereas the materials otherwise prefer to grow in a multilayer fashion $[17,58]$. Another parameter, the PLD deposition temperature, is expected to have an effect on both the diffusivity of adatoms (therefore on the nucleation density) and the re-evaporation probability of species. Higher temperatures increase the probability for adatoms to find a step-edge due to a higher adatom diffusivity. Moreover, it increases the re-evaporation probability of adatoms and a lower sticking coefficient is obtained [243]. However, synthesizing perovskite oxides at higher temperatures (containing the stoichiometry) generally results in an increase in crystalline state and improvement of film properties.

Here, the influence of temperature modulation pulsed laser deposition (TM-PLD) on the growth and sticking of perovskite oxides was investigated. This chapter is organized as follows. In the first section, the theory and background of the sticking coefficient in perovskite oxides are described. Thereafter, the temperature modulation experiment performed to increase sticking of volatile species is explained. In the subsequent section, the experimental and simulation methods used in this chapter are described. Then, the kinetic Monte Carlo simulation and experimental growth results of TM-PLD are presented in the next two sections. The chapter ends with a discussion about the TM-PLD growth of $\mathrm{SrTiO}_{3}$ and $\mathrm{PbTiO}_{3}$ films, and the sticking coefficient of $\mathrm{PbTiO}_{3}$ at constant deposition temperature and after TM-PLD.

\subsection{Sticking coefficient in perovskite oxides}

In this section, the general theory behind the sticking of species during perovskite oxide thin film growth is described.

\subsubsection{Sticking coefficient}

The sticking coefficient $\kappa_{\text {stick }}$ is defined as the ratio between incorporated and deposited elements in a thin film. PLD has the key feature of stoichiometric transfer and often all deposited elements show no difference in sticking. For non-volatile species, it results in a sticking coefficient of (nearly) one. However, for volatile elements the sticking coefficient can become close to zero at elevated temperatures. 
During PLD, particles arrive at the surface, they diffuse over the surface and stick at step-edges of nucleation centers or surface steps. Sometimes particles diffuse without becoming attached to a step-edge and will re-evaporate. Particles with extreme high kinetic energy can either implant or cause re-sputtering of atoms. Both re-evaporation and re-sputtering result in sticking coefficients lower than one. Re-sputtering can be circumvented during PLD by depositing perovskite oxide films in high (oxygen) background pressures and with a relatively low laser fluence [242].

\subsubsection{Re-evaporation}

Atoms are prone to re-evaporate from the surface after arriving from the plasma plume on the substrate. In particular, the probability of volatile species to reevaporate is larger compared to non-volatile species ${ }^{1}$. Lets assume atoms diffuse over a surface for a residence time $\tau_{\text {res }}$ needed to find a step-edge before they re-evaporate from the surface. Then the relation between the average diffusion length $l_{\mathrm{D}}$ and the residence time before re-evaporation $\tau_{\text {res }}$ is given by [3]:

$$
l_{\mathrm{D}}=\sqrt{2 D_{\mathrm{S}} \cdot \tau_{\text {res }}}=\left(2 \nu \alpha^{2} \tau_{\text {res }} e^{-E_{\text {abs }} / k_{\mathrm{B}} T}\right)^{1 / 2}
$$

where $D_{\mathrm{S}}$ is the diffusion coefficient, $\nu$ the attempt frequency, $\alpha$ the characteristic jump distance and $E_{\text {abs }}$ the energy activation barrier for absorption. From Eq. 6.1, it is clear that the deposition temperature $T$ is an important parameter in the sticking of volatile species. On the other hand, an increase in step density $S$ results in a shorter diffusion length $l_{\mathrm{D}}$ of adatoms needed to find a step-edge [127]. The main reason that volatile species re-evaporate is that the average diffusion length $l_{\mathrm{D}}<\rho$, where $\rho$ is the average radius of the area in between growing islands (the perovskite oxide PLD growth kinetics is extensively discussed in Ch. 2 and Ref. [18]).

\subsubsection{Sticking coefficient vs. deposition temperature}

The absorption rate $R_{\text {abs }}$ is defined as the average number of adatoms absorbed per surface area and time. This can be expressed according to Eq. 6.2:

$$
R_{\mathrm{abs}}=\frac{d n}{d t}
$$

\footnotetext{
${ }^{1}$ The residence time $\tau_{\text {res }}$ is assumed to be shorter for volatile species.
} 
The desorption rate $R_{\text {des }}$ is defined as the average number of adatoms desorbed per surface area and per time $\tau_{\text {res }}$ until re-evaporation. The residence time can be calculated according to $\tau_{\text {res }}=1 / \nu \cdot \mathrm{e}^{-E_{\text {des }} / k_{\mathrm{B}} T}$ and therefore $R_{\text {des }}$ is given by:

$$
R_{\mathrm{des}}=\frac{n(t)}{\tau_{\mathrm{res}}}=n(t) \cdot \nu \cdot e^{-E_{\mathrm{abs}} / k_{\mathrm{B}} T}
$$

where, $\mathrm{n}(\mathrm{t})$ is the number of desorbed adatoms. The sticking coefficient is defined $\kappa_{\text {stick }}$ as the difference between the absorption and desorption rate and is expressed as:

$$
\kappa_{\text {stick }}(t)=\frac{R_{\mathrm{abs}}-R_{\mathrm{des}}}{R_{\mathrm{abs}}}=1-\frac{n(t) \cdot \nu}{R_{\mathrm{abs}}} \cdot e^{-E_{\mathrm{abs}} / k_{\mathrm{B}} T}
$$

At temperatures $T$ where species become volatile, the sticking coefficient $\kappa_{\text {stick }}$ decreases to a value in between zero and one ${ }^{2}$. Eq. 6.4 has been used as model to fit the $\mathrm{Pb}$ :Ti ratio (sticking coefficient) measurements.

\subsubsection{Atomic ratio measurement}

Sticking of species is not directly measured. This is due to the fact that it is hard to determine the number of arriving and re-evaporating species. In order to estimate the sticking coefficient, typically the atomic ratio is measured between the $\mathrm{A}$ and $\mathrm{B}$ cations of perovskite oxides in a temperature range of interest ${ }^{3}$. In this study, $\mathrm{PbTiO}_{3}$ is used as system to explore the effect of temperature modulation sequence during PLD on the sticking coefficient. The atomic ratio between $\mathrm{Pb}: \mathrm{Ti}$ is $1: 1$, when the sticking of $\mathrm{Pb}$ is similar to the sticking of $\mathrm{Ti}^{4}$. However, $\mathrm{Pb}$ starts to become volatile $\approx 600{ }^{\circ} \mathrm{C}[127]$. Measuring the ratio between $\mathrm{Pb}: \mathrm{Ti}$ in the temperature range below the $\mathrm{Ti}$ evaporation temperature indicates at which temperature $\mathrm{Pb}$ starts to re-evaporate or not.

\subsection{Experiment}

Temperature modulation pulsed laser deposition (TM-PLD) is proposed here as a method to extend the deposition temperature of volatile perovskite oxides. The

\footnotetext{
${ }^{2}$ Non-volatile species have a sticking coefficient $\kappa_{\text {stick }}=1$.

${ }^{3}$ Note that this is only valid when at least one of the A and B cations does not re-evaporate.

${ }^{4}$ In the case of stoichiometric transfer of species from target to substrate.
} 


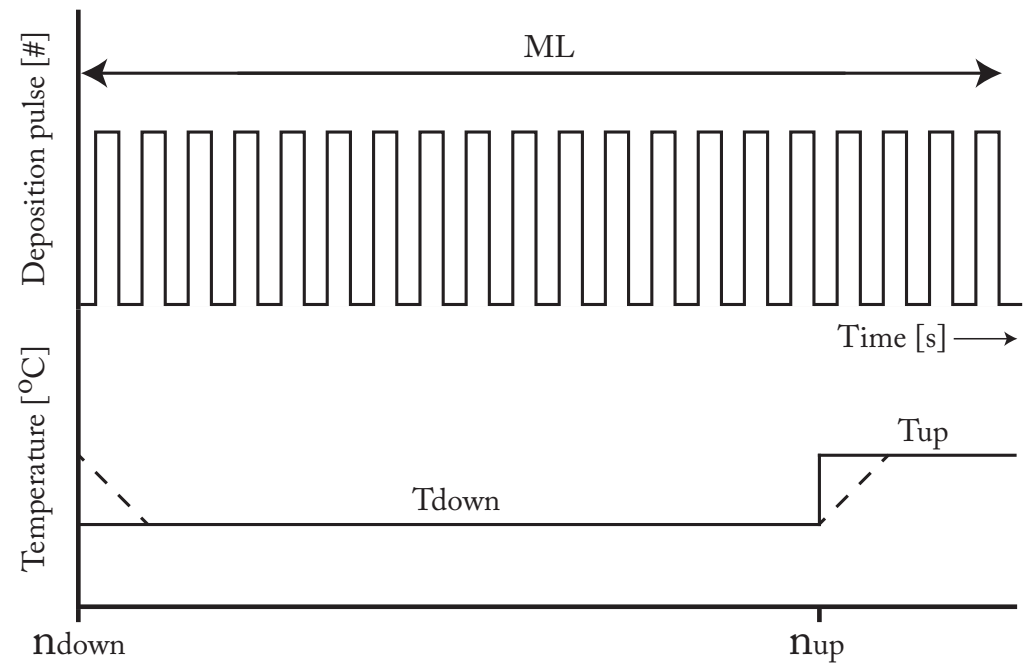

Fig. 6.1 TM-PLD illustrated by a instantaneously and ramp-rate (dashed line) temperature interval during ML (assuming 20 pulses) deposition. The temperature is increased from $T_{\text {down }}$ to $T_{\text {up }}$ after pulse number $n_{\text {up }}$ in a ML (number in between $0-20$ ) and the temperature is decreased from $T_{\text {up }}$ to $T_{\text {down }}$ after pulse number $n_{\text {down }}$ in a ML (number in between 0 - 20).

method differs from pulsed laser interval deposition (PLID) as the PLD growth takes place at two modulating growth temperatures instead of deposition rate modulation $[44,58]$. TM-PLD is based on achieving a high nucleation density $N_{\mathrm{s}}$ in the first growth stage of a single monolayer (ML) and is aiming for a larger diffusion length $l_{\mathrm{D}}$ at the final part of a ML growth stage. This is done to promote interlayer mass transport. Eq. 6.5 gives the relation (for defect-free surfaces) between the nucleation density $N_{\mathrm{s}}$, deposition rate $F$ and adatom diffusion coefficient $D_{\mathrm{S}}$ [244]:

$$
N_{\mathrm{s}}=\left(F / D_{\mathrm{S}}\right)^{x}
$$

where the exponent $x$ is the scaling order and depends on the smallest stable nucleation size. Starting the first growth stage of a ML at lower $T$ results in a decrease of $D_{\mathrm{S}}$ and $l_{\mathrm{D}}$, see Eq. 6.1. From Eq. 6.5, it becomes clear that a larger $N_{\mathrm{s}}$ is obtained having a lower $D_{\mathrm{S}}$ at lower $T$. The increase in nucleation density results in an increase in step density $S$ and decreases the radius of the area between islands $\rho$. Therefore, the probability for volatile species to find a step-edge is increased in the first part of the monolayer and sticking should be enhanced. When the island diameter is equal to the length of the area in between 
islands, island coalescence takes place and $l_{\mathrm{D}}$ is not large enough for interlayer mass transport and perfect $2 \mathrm{D}$ growth does not occur. By increasing $T$ at $n_{\text {up }}$ (pulse number in a ML, whereafter the temperature is increased from $T_{\text {down }}$ to $T_{\text {up }}$ ), the $D_{\mathrm{S}} / l_{\mathrm{D}}$ is increased and the diffusion of adatoms overcomes the average radius of islands $r_{\text {island }}$ [18].

Fig. 6.1 represents an illustrated scheme of TM-PLD during a ML. The scheme is based on $\mathrm{SrTiO}_{3}$ homoepitaxy, where in typical PLD parameters 20 pulses are needed to deposit one ML, see Refs. [44, 58]. In Fig. 6.1, a TM-PLD sequence is presented, where the first part of a ML is (mainly) deposited at the lower temperature $T_{\text {down }}$ after decreasing the temperature from deposition pulse number $n_{\text {down }}$. After several deposition pulses, the temperature is increased up to $T_{\text {up }}$ from deposition pulse $n_{\text {up }}{ }^{5}$. This temperature sequence in a ML has been used throughout this chapter. $\mathrm{SrTiO}_{3}$ has been used as perovskite oxide system to study the influence of TM-PLD on the step density $S$, where $\mathrm{PbTiO}_{3}$ is utilized for exploring the effect of TM-PLD on the $\mathrm{Pb}$ :Ti ratio and sticking of $\mathrm{Pb}$.

\subsection{Methods}

Kinetic Monte Carlo (KMC) simulations were performed to gain growth understanding and to elucidate the TM-PLD growth experiments. Note that the KMC simulations are utilized to study the effect of TM-PLD on the step density $S$, whereas any volatility of the adatoms is not taken into account. A detailed description of the KMC simulation algorithm is described elsewhere [148]. A lattice grid of $512 \times 128$ u.c. $^{2}$ was used in the KMC simulations ${ }^{6}$ (in this work, the algorithm was modified such that the temperature is defined per deposition pulse). Two temperatures are included, namely $T_{\text {up }}$ the higher temperature of the temperature interval and $T_{\text {down }}$ the lower temperature of the temperature interval. The pulse number $n_{\text {up }}$ is the deposition pulse number, whereafter the deposition temperature is increased from $T_{\text {down }}$ to $T_{\text {up }}$ and this is reversed for $n_{\text {down }}$. The values used for the parameters in the KMC simulation have been extracted from other work: $E_{\mathrm{D}}=2.2 \mathrm{eV}, E_{\mathrm{N}}=0.27 \mathrm{eV}, k_{0}=10^{13} \mathrm{~Hz}, \phi=0^{\circ}$ and $f_{\text {rep }}=1 \mathrm{~Hz}[17,18,58]$. Two types of temperature functions have been included separately in the KMC algorithm. First, a step function having an infinite ramp-rate, where the temperature increases instantaneously from the lower temperature $T_{\text {down }}$ to the higher temperature $T_{\text {up }}$. Second, as the heater has a finite ramp-rate, a typical ramp-rate of $20{ }^{\circ} \mathrm{C} / \mathrm{s}$ has been included in the $\mathrm{KMC}$ algorithm in order to get more realistic

\footnotetext{
${ }^{5}$ Time between a constant $n_{\text {down }}$ and $n_{\text {up }}$ varies with PLD repetition rate $f_{\text {rep }}$.

${ }^{6}$ Here, u.c. stands for unit cell.
} 
results ${ }^{7}$.

$\mathrm{SrTiO}_{3}$ and $\mathrm{PbTiO}_{3}$ thin films were deposited by PLD in the COMAT system. The COMAT system is equipped with a COHERENT ArF-KrF laser (wavelength 248 $\mathrm{nm}$ ) suitable for deposition of perovskite oxide thin films. A recently developed infrared laser system was utilized in order to apply a temperature modulation and/or fast heating/cooling sequence during growth. The infrared laser system contains a $120 \mathrm{~W}$ Coherent Quattro FAP laser for direct heating. The maximum temperature can be increased up to $1100{ }^{\circ} \mathrm{C}$ with a maximum ramp-rate ${ }^{8}$ of $20{ }^{\circ} \mathrm{C} / \mathrm{s}$. A typical spot of $1 \mathrm{~cm}$ was aligned on the backside of the sample plate. To obtain near black body absorption, the backside was roughened and oxidized. An infrared pyrometer (temperature accuracy $\pm 3{ }^{\circ} \mathrm{C}$ at $710^{\circ} \mathrm{C}$ ) was used to measure and control the temperature of the sample plate.

The $\mathrm{SrTiO}_{3}$ substrates were prepared using a standard procedure to obtain $\mathrm{TiO}_{2}$ terminated surfaces [19]. $\mathrm{SrTiO}_{3}$ homoepitaxial films were deposited using a laser fluence $=1.3 \mathrm{~J} / \mathrm{cm}^{2}$, spotsize $=2.35 \mathrm{~mm}^{2}$, oxygen pressure $\mathrm{PO}_{2}=0.1 \mathrm{mbar} \mathrm{O}_{2}$ and repetition rate $f_{\text {rep }}=0.5 \mathrm{~Hz}$ (It is more easy to maintain $n_{\text {down }}$ and $n_{\text {up }}$ manually at $f_{\text {rep }}=0.5 \mathrm{~Hz}$ instead of $f_{\text {rep }}=1 \mathrm{~Hz}$, where the latter has been used in the KMC simulations). $\mathrm{PbTiO}_{3}$ was chosen as the material containing a volatile element, namely $\mathrm{Pb}$. These heteroepitaxial films were deposited using a laser fluence $=2.0 \mathrm{~J} / \mathrm{cm}^{2}$, spotsize $=2.35 \mathrm{~mm}^{2}, f_{\text {rep }}=0.5 \mathrm{~Hz}$ and $\mathrm{PO}_{2}=0.14$ mbar $\mathrm{O}_{2} . \mathrm{SrRuO}_{3}$ has been deposited as buffer layer for inducing layer-by-layer growth of $\mathrm{PbTiO}_{3}$ films. The deposition conditions of $\mathrm{SrRuO}_{3}$ were similar to that of $\mathrm{PbTiO}_{3}$.

In situ reflection high energy electron diffraction (RHEED) was used to monitor the step density $S$ during growth as the RHEED specular intensity $I_{\text {RHEED }}$ scales with $S$ according to $I_{\text {RHEED }} \sim 1-\frac{S}{S_{\max }}$, see Eq. 2.5 [16]. Subsequently, in situ XPS was performed on an Omicron nanotechnology $\mathrm{GmbH}$ system using a $\mathrm{Al} k \alpha$ x-ray source (XM1000) in a background pressure of $5 \times 10^{-11}$ mbar. XPS analysis was used to study the atomic ratio. Data analysis was done using a 7 channel EA 125 electron analyzer in CAE mode. The effective peak area was determined by using a Gaussian fit after a Shirley background subtraction. Ex situ AFM topography images were recorded in Tapping Mode (TM) using a Bruker Dimension ICON AFM and a Bruker TESPA doped Si cantilever $f_{0}=320 \mathrm{kHz}$. The AFM images were used in order to study the surface topography after PLD.

\footnotetext{
${ }^{7}$ A temperature ramp-rate of $20^{\circ} \mathrm{C} / \mathrm{s}$ has been found for the laser heater used in the experiments.

${ }^{8}$ Software infrared thermometer has been used for determination of the (maximum) laser heater ramp-rate.
} 


\subsection{Kinetic Monte Carlo simulations}

Kinetic Monte Carlo (KMC) simulations ${ }^{9}$ have been conducted in order to study the effects of TM-PLD on the growth and step density $S$ during PLD of perovskite oxides.

\subsubsection{TM-PLD (instantaneously)}

KMC simulation results after an instantaneous increase/decrease in temperature during TM-PLD are shown below.

\section{Temperature sequence}

KMC simulations are performed in order to verify whether the step density $S$ could be strongly modulated by applying TM-PLD and which temperature sequence is required during TM-PLD. Fig. 6.2 shows the RHEED specular intensity during and surface topography images after the deposition of 10 ML's using TM-PLD. RHEED oscillations are observed for $T_{\text {down }}=710{ }^{\circ} \mathrm{C}, T_{\text {up }}=850{ }^{\circ} \mathrm{C}, n_{\text {down }}=0$ and $n_{\mathrm{up}}=19$ (left panel). Therefore, the step density $S$ is modulating during this TM-PLD sequence [18]. For this KMC simulation, the amplitude of the RHEED specular intensity oscillation $\approx 0.15 I / I_{0}$. The maximum $I_{\text {RHEED }}$ decreases with the number of deposited ML's. The exponential decay of maximum $I_{\text {RHEED }}$ becomes smaller and a smoother surface will be obtained when $T_{\text {down }}$ is decreased. The terrace structure, small islands and vacancy islands are visible in the KMC surface topography after the deposition of 10 ML's. Different results are obtained when $n_{\text {down }}=19$ and $n_{\text {up }}=0$ (right panel). The RHEED specular intensity $I_{\text {RHEED }}$ does not recover in the latter deposition pulses in a ML. From this observation, it becomes clear that the RHEED specular intensity $I_{\text {RHEED }}$ keeps decreasing and $S$ keeps increasing with almost every deposition pulse. The KMC surface topography image confirms that it results in $3 \mathrm{D}$ growth and the terrace structure is moreless disappeared after the deposition of $10 \mathrm{ML}$ 's.

\section{RHEED specular intensity vs. $n_{\text {up }}$}

Figs. 6.3 (a-d) show the KMC simulated RHEED specular intensities during PLD at constant temperatures of a) $T=600{ }^{\circ} \mathrm{C}$ and $T=850{ }^{\circ} \mathrm{C}$, and during TM-PLD deposition at $T_{\text {down }}=600{ }^{\circ} \mathrm{C}$ and $T_{\text {up }}=850{ }^{\circ} \mathrm{C}$ for different $n_{\text {up }}$, namely b) $n_{\text {up }}=1$, c) $n_{\text {up }}=10$ and d) $n_{\text {up }}=19$. The RHEED specular intensity gradually decreases with every ML at both $T=600{ }^{\circ} \mathrm{C}$ and $T=850{ }^{\circ} \mathrm{C}$. An amplitude of $<0.1 I / I_{0}$ has been found for the RHEED specular intensity oscillation of the

\footnotetext{
${ }^{9}$ The basics of KMC simulations are explained in Ch. 2.
} 


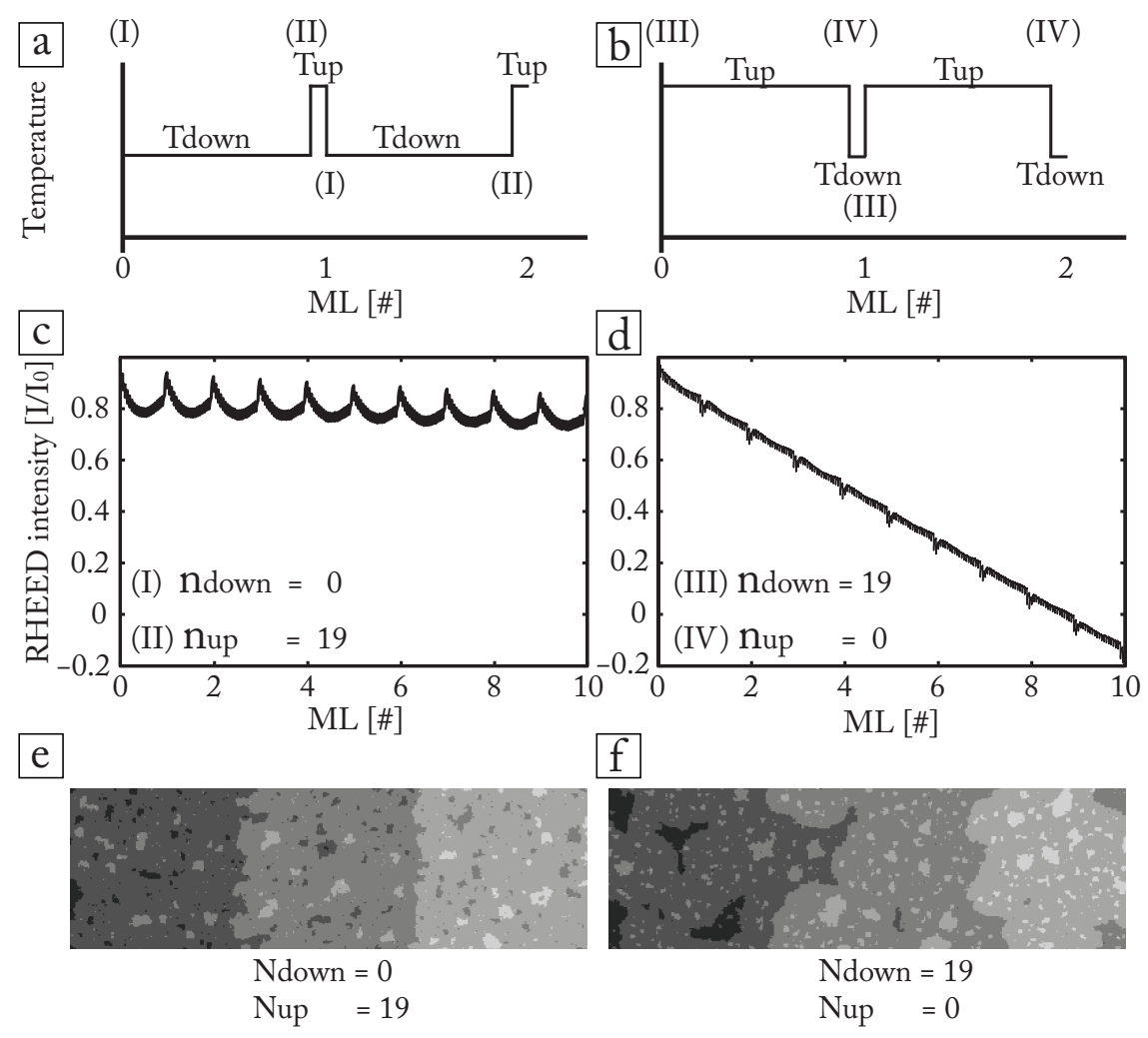

Fig. 6.2 KMC simulations of TM-PLD growth for different temperature sequences using $T_{\text {down }}=710{ }^{\circ} \mathrm{C}$ and $T_{\text {up }}=850{ }^{\circ} \mathrm{C}$, a) $n_{\text {down }}=0$ and $n_{\text {up }}=19$, b) $n_{\text {down }}=19$ and $n_{\text {up }}=0$, c) RHEED specular intensity $I / I_{0}$ for $n_{\text {down }}=0$ and $n_{\text {up }}=19$, d) RHEED specular intensity $I / I_{0}$ for $n_{\text {down }}=19$ and $n_{\text {up }}=0$, e) KMC surface topography after the growth of 10 ML's for $n_{\text {down }}=0$ and $n_{\text {up }}=19$ and f) KMC surface topography after the growth of $10 \mathrm{ML}$ 's for $n_{\text {down }}=19$ and $n_{\text {up }}=0$.

KMC simulation at $T=850^{\circ} \mathrm{C}$, while hardly oscillations have been observed at $T=600{ }^{\circ} \mathrm{C}$.

The RHEED specular intensity during TM-PLD varies with $n_{\text {up }}$. Varying $n_{\text {up }}$ affects the shape of the RHEED specular intensity oscillations and its recovery of intensity after every ML. Selecting $n_{\text {up }}=1$ still results in a similar shape of the RHEED specular intensity compared to deposition at $T=850^{\circ} \mathrm{C}$, see Fig. $6.2 \mathrm{a}$ ). Both simulations are performed for $T=850{ }^{\circ} \mathrm{C}$ over almost the entire ML. The amplitude of the oscillations is similar to the deposition at a constant $T=850{ }^{\circ} \mathrm{C}$. A spike is observed during the first deposition pulse of the monolayer. The step density $S$ is increased as adatoms are less mobile at a $T=600{ }^{\circ} \mathrm{C}$. 

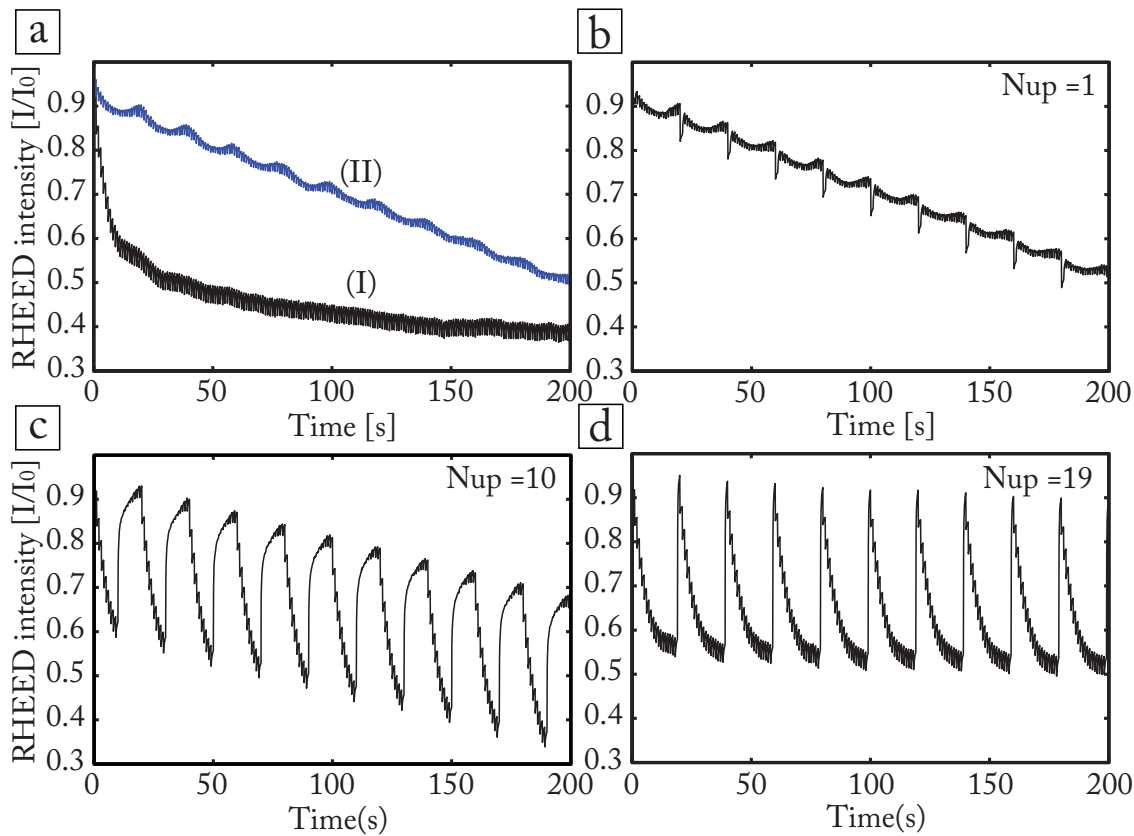

Fig. 6.3 KMC simulation of RHEED specular intensity for different $n_{\text {up }}$ at $T_{\text {down }}=600{ }^{\circ} \mathrm{C}, T_{\text {up }}=850{ }^{\circ} \mathrm{C}$, a) (I) at constant temperatures $T=600{ }^{\circ} \mathrm{C}$ and (II) at $T=850{ }^{\circ} \mathrm{C}$, b) $n_{\text {up }}=1$, c) $n_{\text {up }}=10$, d) $n_{\text {up }}=19$.

However, when $n_{\text {up }}=10$ (see Fig. $6.3 \mathrm{c}$ )) the shape and recovery of the RHEED specular intensity oscillations changes. The first half ML and the second half ML are grown at $T_{\text {down }}=600{ }^{\circ} \mathrm{C}$ and at $T_{\text {up }}=850{ }^{\circ} \mathrm{C}$, respectively. RHEED oscillations are visible with an amplitude of $\approx 0.3 I / I_{0}$, which is larger than the $<0.1 \mathrm{I} / I_{0}$ found at $T=850{ }^{\circ} \mathrm{C}$. The oscillations are asymmetric in shape and are furthermore indicative for a larger $S$ at half $\mathrm{ML}$ compared to deposition at $T=850{ }^{\circ} \mathrm{C}$. A recovery of the RHEED specular intensity is obtained starting from the moment $T=T_{\text {up }}$. After the deposition of every subsequent ML, the maximum $I_{\text {RHEED }}$ decays as it is not completely recovered. The shape of the RHEED oscillations are similar to what has been obtained for pulsed laser interval deposition (PLID) KMC simulations [58].

Total recovery of the RHEED specular intensity has been observed when $n_{\text {up }}=19$ and therefore no decay of the maximum $I_{\text {RHEED }}$ is visible. From Fig. $6.3 \mathrm{~d}$ ), it can be concluded that the RHEED specular intensity oscillations shape is different compared to $n_{\text {up }}=10$. Between deposition pulses 11 and 19, the RHEED specular intensity decreases and therefore $S$ is still increasing. The amplitude of the RHEED oscillations is $>0.3 I / I_{0}$, a similar value which has been obtained for 


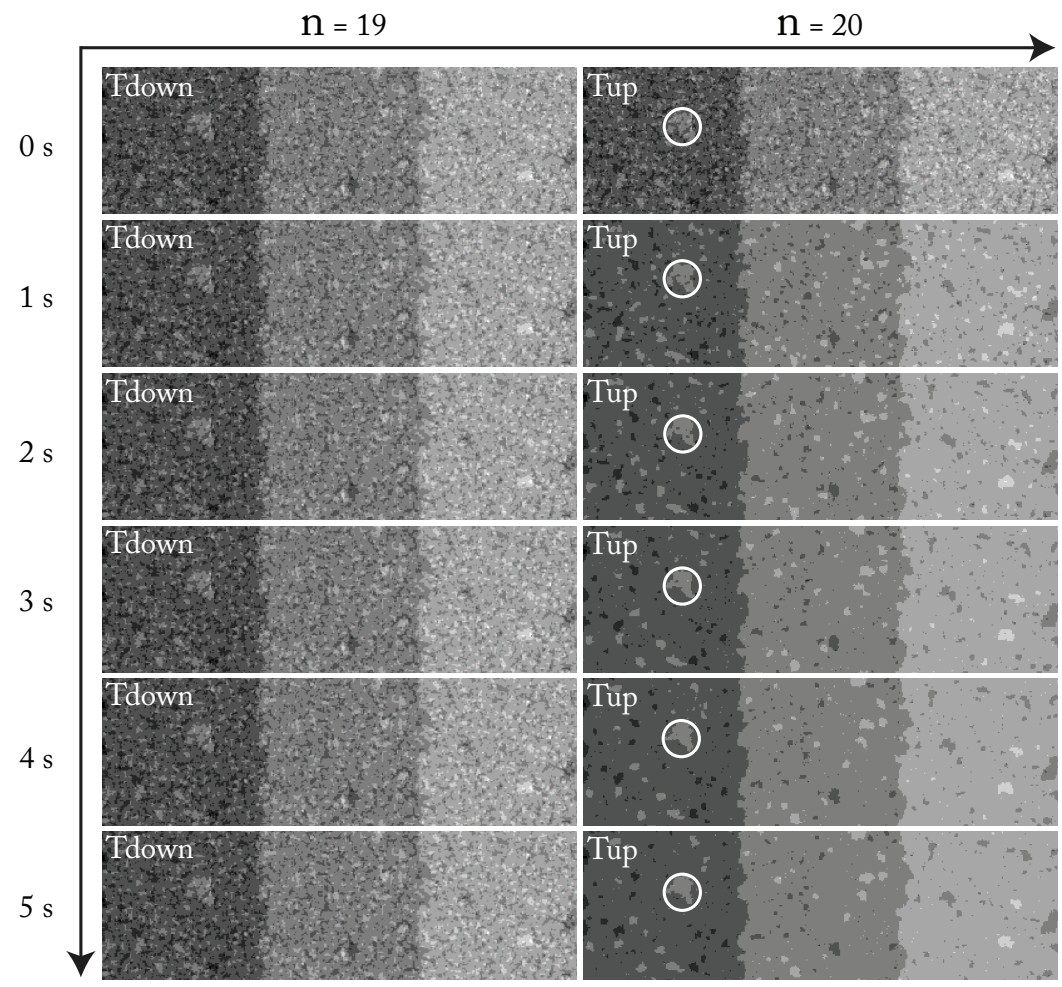

Fig. 6.4 KMC simulated surface topography images during TM-PLD of pulse $n=19$ and 20 of the tenth ML deposition with $n_{\text {up }}=19, T_{\text {down }}=600{ }^{\circ} \mathrm{C}$ and $T_{\text {up }}=800{ }^{\circ} \mathrm{C}$. Time $t$ should be read from top to bottom followed by the number of deposition pulses $n$ from left to right.

$n_{\text {up }}=10$. Other simulations revealed that increasing $T_{\text {up }}$ results in an increase of RHEED specular intensity recovery for KMC simulations, where no total recovery is obtained for a certain $n_{\text {up }}$.

\section{Final growth stage ML}

Fig. 6.4 shows the results of a $\mathrm{KMC}$ simulation using the following parameters: $f_{\text {rep }}=0.2 \mathrm{~Hz}, \operatorname{ML}(\#)=10, T_{\text {down }}=600{ }^{\circ} \mathrm{C}, T_{\text {up }}=800{ }^{\circ} \mathrm{C}$ and $n_{\text {up }}=19$ (meaning $T=800{ }^{\circ} \mathrm{C}$ from pulse 20$)^{10}$. KMC surface topography images were selected during deposition and relaxation of pulses 19 and 20, both in intervals of one second. Deposition of pulse number $20(n=20)$ occurs at $t=0 \mathrm{~s}$. At this time, islands are visible on the terraces. From this moment, $T_{\text {down }}$ is changed to $T_{\text {up }}=800{ }^{\circ} \mathrm{C}$. When $t=1 \mathrm{~s}$, the smallest islands disappeared as the diffusion

\footnotetext{
${ }^{10} \mathrm{~A}$ repetition rate of $f_{\text {rep }}=0.2 \mathrm{~Hz}$ is chosen as there is more time between the deposition pulses in order to study relaxation.
} 
coefficient of adatoms increases significantly. Adatoms diffuse and some become attached in vacancy islands. Others seem to become attached to other adatoms forming islands. Interlayer mass transport is visible in Fig. 6.4, when one follows the island and adatoms surrounded by the white circular marker in time $t$. After $t=1 \mathrm{~s}$, a few adatoms diffuse to become attached to vacancy islands and/or islands. Most of the mass transport seems to happen from $0<t \leq 1$.

\subsubsection{TM-PLD ramp-rate}

KMC simulation results after a more realistic ramp-rate temperature increase and decrease during TM-PLD are shown below.

\section{Ramp-rate}

Fig. 6.5 shows the results of a KMC simulation using the following parameters: $f_{\text {rep }}=1 \mathrm{~Hz}, \operatorname{ML}(\#)=10, T_{\text {down }}=600{ }^{\circ} \mathrm{C}, T_{\text {up }}=710{ }^{\circ} \mathrm{C}$ and $n_{\text {up }}=13$. A ramp-rate of $20^{\circ} \mathrm{C} / \mathrm{s}$ was implemented and therefore it takes 5 - 6 pulses before the temperature increases from $T_{\text {down }}=600{ }^{\circ} \mathrm{C}$ to $T_{\text {up }}=710{ }^{\circ} \mathrm{C}$. RHEED specular intensity oscillations are still clearly visible using the ramp-rate. In this specific simulation, the amplitude is $\approx 0.2 I / I_{0}$ of the RHEED specular intensity oscillations. The shape of the oscillations is different compared to instantaneously increase of temperature as the recovery of the RHEED specular intensity takes more time. The recovery is such that the TM-PLD RHEED specular intensity does not recover to the maximum RHEED specular intensity $I_{\text {RHEED }}$ of the deposition at $T=710{ }^{\circ} \mathrm{C}$. From the KMC surface topography image after TM-PLD, it becomes clear that islands have a high density and are smaller compared to the deposition at $T=710{ }^{\circ} \mathrm{C}$. Therefore, most likely the surface contains a higher step density $S$.

\section{RHEED specular intensity vs. $n_{\text {up }}$}

The previous KMC simulation was repeated for different $n_{\text {up }}$. These results are presented in Fig. 6.5 as well. From these KMC simulation results, it can be seen that $n_{\text {up }}$ has a influence on the RHEED specular intensity. Varying $n_{\text {up }}$ moreless changes the "offset" of the RHEED specular intensity. When $n_{\text {up }}=13$, the maximum $I_{\text {RHEED }}$ is not recovered to the maximum $I_{\text {RHEED }}$ at $T=710{ }^{\circ} \mathrm{C}$, while for $n_{\text {up }}=8$ the maximum $I_{\text {RHEED }}$ does recover. As the "offset" of the RHEED specular intensity changes with $n_{\text {up }}$, it seems that a higher maximum $S$ (minimum $I_{\text {RHEED }}$ ) can be obtained while having a lower maximum $I_{\text {RHEED }}$. 

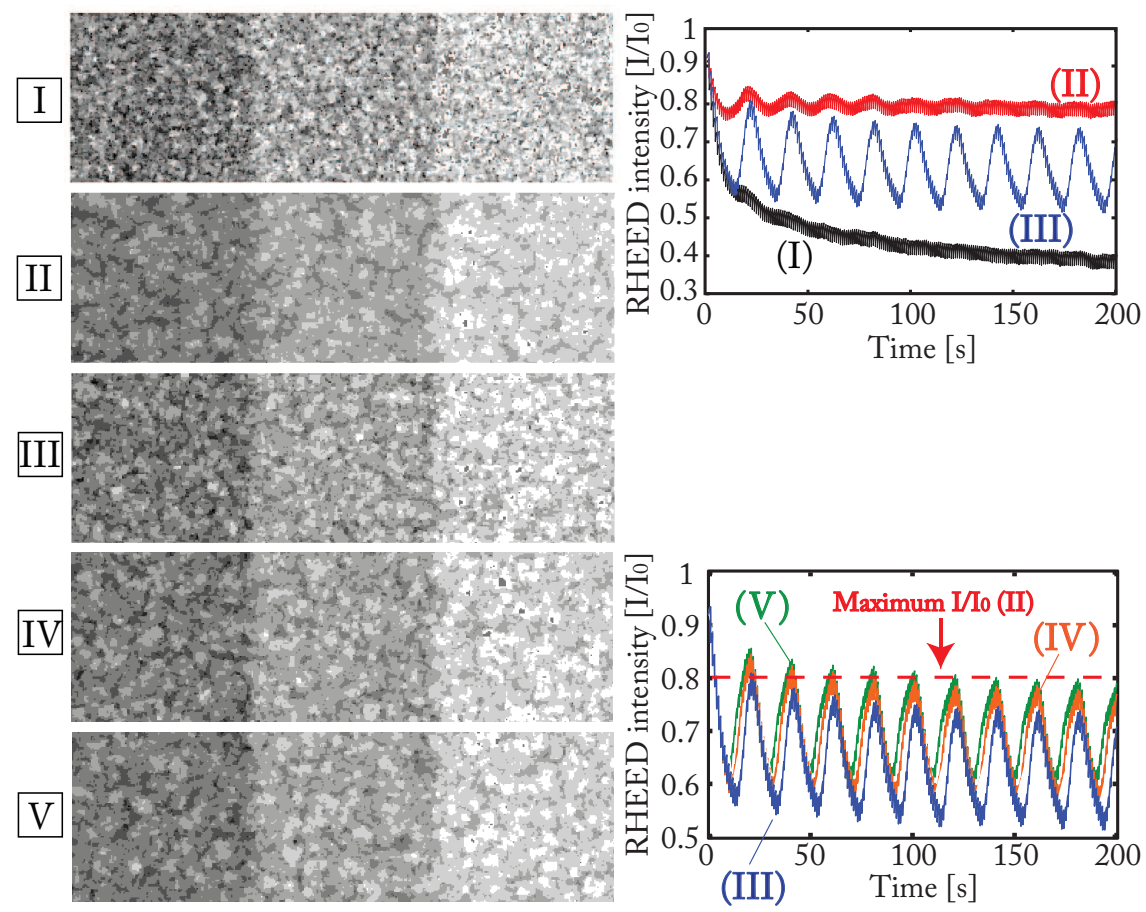

Fig. 6.5 KMC simulation of RHEED specular intensity and surface topography for (I) $T=600{ }^{\circ} \mathrm{C}$, (II) $T=710{ }^{\circ} \mathrm{C}$, (III) $n_{\text {up }}=13, T_{\text {down }}=600{ }^{\circ} \mathrm{C}$ and $T_{\text {up }}=710{ }^{\circ} \mathrm{C}$, (IV) $n_{\text {up }}=10, T_{\text {down }}=600{ }^{\circ} \mathrm{C}$ and $T_{\text {up }}=710{ }^{\circ} \mathrm{C}$ and $(\mathrm{V}) n_{\text {up }}=8, T_{\text {down }}=600{ }^{\circ} \mathrm{C}$ and $T_{\text {up }}=710^{\circ} \mathrm{C}$. 


\subsection{Experimental results}

Experiments have been conducted to study the influence of TM-PLD on the growth kinetics. Furthermore, experiments have been performed to measure the atomic ratio between $\mathrm{Pb}$ :Ti.

\subsubsection{RHEED $\mathrm{SrTiO}_{3}$ homoepitaxial growth}

Fig. 6.6 presents the RHEED specular intensities obtained during $\mathrm{SrTiO}_{3}$ homoepitaxial PLD growth experiments. $\mathrm{SrTiO}_{3}$ films have been grown at constant temperatures of $T=600{ }^{\circ} \mathrm{C}$ (see Fig. 6.6 a) and $T=710^{\circ} \mathrm{C}$ (see Fig. 6.6 b). In both experiments, RHEED specular intensity oscillations have been observed, where the maximum $I_{\text {RHEED }}$ mutes with (almost) every deposited ML. Dashed (red) lines are included in Fig. 6.6 to indicate the average maximum RHEED specular intensity $\left(0.69 I_{0}\right)$ and the average minimum RHEED specular intensity $\left(0.36 I_{0}\right)$ of the TMPLD film, where $I_{0}$ is $I_{\text {RHEED }}$ before deposition. The maximum RHEED specular intensity is below $0.69 I_{0}$ for all $\mathrm{ML}$ deposited at $T=600{ }^{\circ} \mathrm{C}$. At $T=710{ }^{\circ} \mathrm{C}$, only $4 \mathrm{ML}$ are grown where the RHEED specular intensity recovers above $0.69 I_{0}$. Both samples show a decay of the RHEED specular intensity due to roughening of the surface (increase of $S$ ) as discussed in Ch. 2. The minimum RHEED specular intensity decreases after $t \approx 500 \mathrm{~s}$ below $0.36 I_{0}$. From $t \approx 500 \mathrm{~s}$, the average RHEED specular intensity is below $0.36 I_{0}$ for both $T=600{ }^{\circ} \mathrm{C}$ and $T=710{ }^{\circ} \mathrm{C}$. An amplitude of $0.33 I_{0}$ has been found for the TM-PLD sample, where (at $t=1000 \mathrm{~s}$ ) $0.06 I_{0}$ and $0.07 I_{0}$ have been determined for $T=600{ }^{\circ} \mathrm{C}$ and $T=710^{\circ} \mathrm{C}$, respectively.

In Fig. 6.7, a zoom-in of the RHEED specular intensity is shown for the samples grown at $T=710{ }^{\circ} \mathrm{C}$ and $T_{\text {down }}=600{ }^{\circ} \mathrm{C} / T_{\text {up }}=710{ }^{\circ} \mathrm{C}\left(n_{\text {down }}=0\right.$ and $n_{\text {up }}=15-$ 16). The shape of the RHEED specular intensity oscillation of $\mathrm{SrTiO}_{3}$ deposited at $T=710{ }^{\circ} \mathrm{C}$ is similar to what has been observed previously (see Fig. 3.2 a) of Ref. [18]). During TM-PLD, the shape of the RHEED oscillations is different compared to the shape of the RHEED oscillations recorded at $T=710{ }^{\circ} \mathrm{C}$. After pulse $n_{\text {down }}$, the RHEED specular intensity drops rapidly as $T$ and $l_{\mathrm{D}}$ decrease and therefore the step density $S$ is higher compared to the step density at a constant temperature of $T=710{ }^{\circ} \mathrm{C}$. The reverse is observed after $n_{\text {up }}$. Here, the RHEED specular intensity increases rapidly as $T$ and $l_{\mathrm{D}}$ increases and the step density rapidly decreases as islands grow in lateral size. 


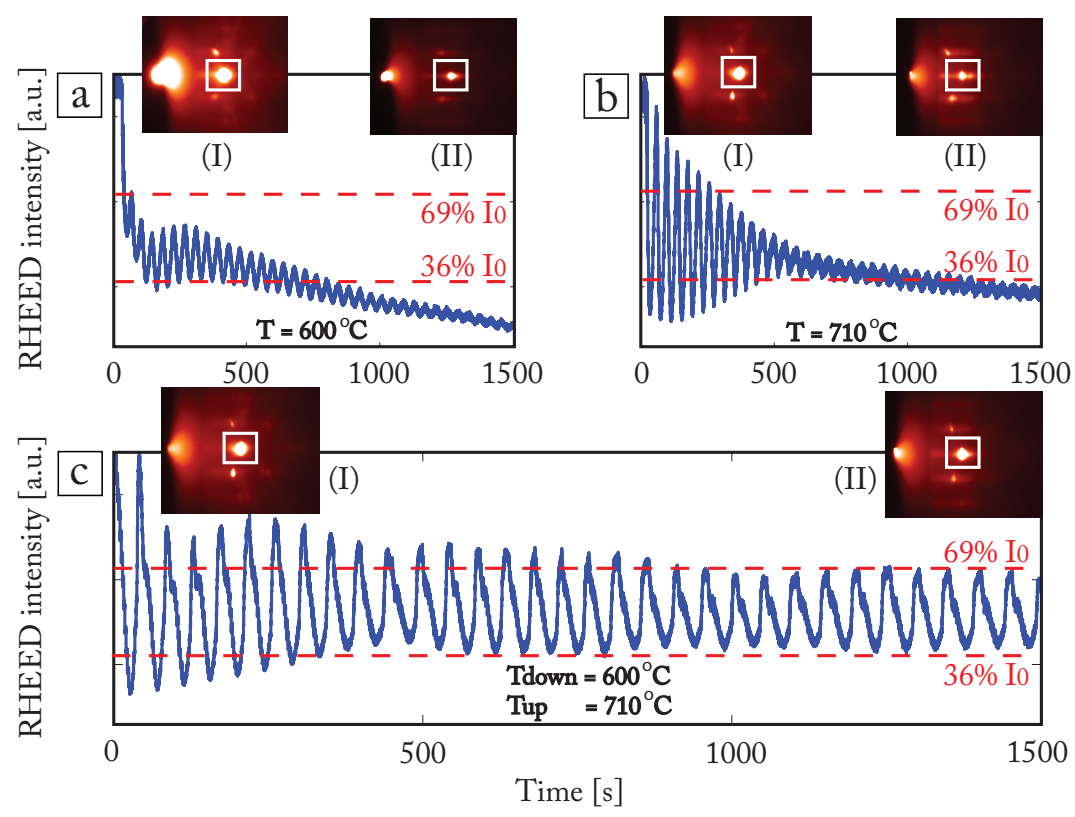

Fig. 6.6 RHEED specular intensity and diffraction pattern (I) before and (II) after experimental $\mathrm{SrTiO}_{3}$ homoepitaxial growth at $\mathrm{PO}_{2}=0.1$ mbar $\mathrm{O}_{2}$, a) $\mathrm{T}=600{ }^{\circ} \mathrm{C}$, b) $T=710{ }^{\circ} \mathrm{C}$ and c) $T_{\text {down }}=600, T_{\text {up }}=710{ }^{\circ} \mathrm{C}, n_{\text {down }}=0$ and $n_{\text {up }}=15-16$.

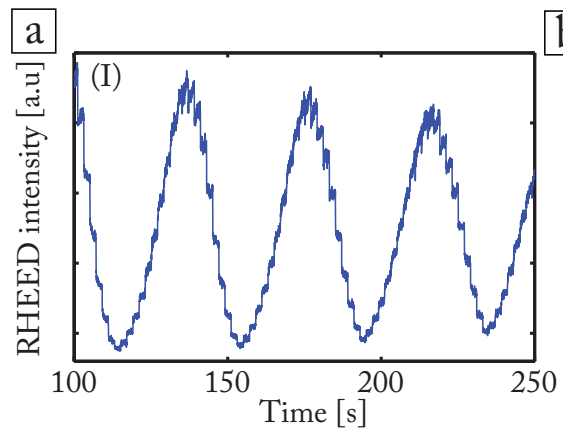

b

$\mathrm{T}$

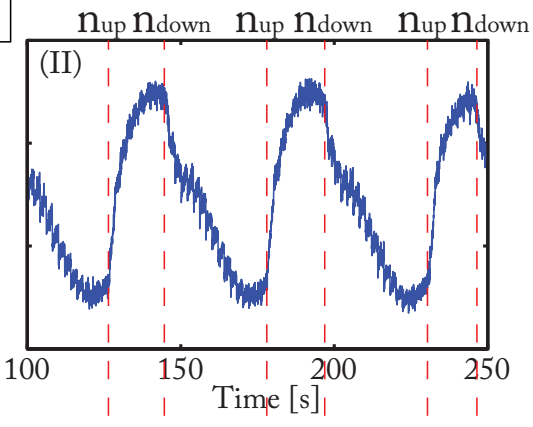

(I) $\mathrm{T}=710^{\circ} \mathrm{C}$

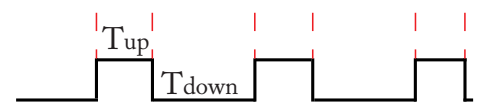

$$
\text { (II) } \begin{aligned}
\text { Tdown } & =600^{\circ} \mathrm{C} \\
\text { Tup } & =710^{\circ} \mathrm{C}
\end{aligned}
$$

Fig. 6.7 Zoom-in RHEED specular intensity during experimental $\mathrm{SrTiO}_{3}$ homoepitaxial growth at $\mathrm{PO}_{2}=0.1$ mbar $\mathrm{O}_{2}$, a) $T=710{ }^{\circ} \mathrm{C}$, b) $T_{\text {down }}=600, T_{\text {up }}=710{ }^{\circ} \mathrm{C}, n_{\text {down }}=0$ and $n_{\text {up }}=15-16$. 


\subsubsection{AFM $\mathrm{SrTiO}_{3}$ homoepitaxial growth}

Ex situ TM-AFM images have been taken after deposition of $\mathrm{SrTiO}_{3}$ films with a thickness of $100 \mathrm{MLs}^{11}$. Fig. 6.8 shows the surface topography of $\mathrm{SrTiO}_{3}$ surfaces after deposition at a) $T=600{ }^{\circ} \mathrm{C}$, b) $T=710{ }^{\circ} \mathrm{C}$ and c) $T_{\text {down }}=600$, $T_{\text {up }}=710^{\circ} \mathrm{C}$. A terrace like structure is visible for all surfaces after deposition of 100 MLs. RMS roughness values $\sigma_{\mathrm{RMS}}$ have been measured of $\sigma_{\mathrm{RMS}}=0.37 \mathrm{~nm}$ $\left(T=600{ }^{\circ} \mathrm{C}\right), \sigma_{\mathrm{RMS}}=0.18 \mathrm{~nm}\left(T=710{ }^{\circ} \mathrm{C}\right)$ and $\sigma_{\mathrm{RMS}}=0.19 \mathrm{~nm}\left(T_{\text {down }}=600{ }^{\circ} \mathrm{C}\right.$, $\left.T_{\text {up }}=710{ }^{\circ} \mathrm{C}\right) . \mathrm{SrTiO}_{3}$ substrate surfaces after an HF treatment and annealing procedure have RMS values ranging from $0.14-0.18 \mathrm{~nm}$. Where the RMS values are quite similar for the surfaces in Figs. $6.8 \mathrm{~b}$ ) and $6.8 \mathrm{c}$ ), the step-edges of the surfaces are clearly different. The step-edges are disordered for the surface grown in Fig. 6.8 b), while this is not the case in Fig. 6.8 c).
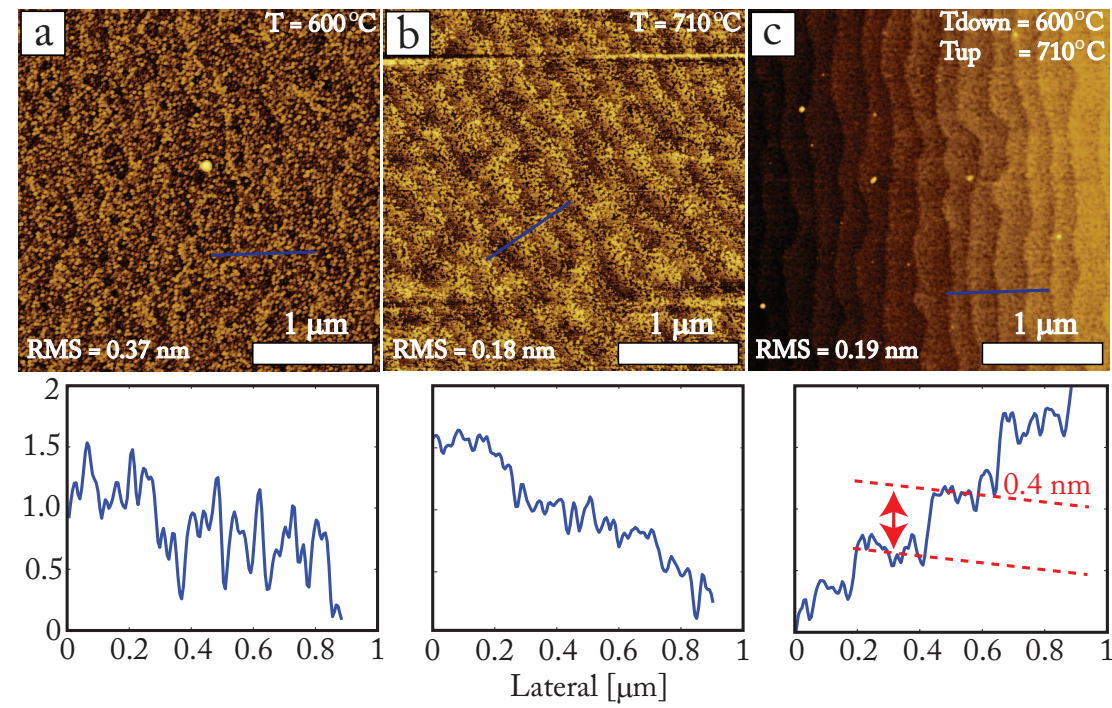

Fig. 6.8 Ex situ TM-AFM images taken after $100 \mathrm{ML}$ 's of $\mathrm{SrTiO}_{3}$ homoepitaxial growth, a) $T=600{ }^{\circ} \mathrm{C}$, b) $T=710{ }^{\circ} \mathrm{C}$ and c) $T_{\text {down }}=600{ }^{\circ} \mathrm{C}, T_{\text {up }}=710{ }^{\circ} \mathrm{C}, n_{\text {down }}=0$ and $n_{\text {up }}=15-16$.

\subsection{3 $\mathrm{PbTiO}_{3}$ growth}

$\mathrm{PbTiO}_{3}$ films are grown on $\mathrm{TiO}_{2}$ terminated $\mathrm{SrTiO}_{3}$ substrates and on $10 \mathrm{~nm}$ thick $\mathrm{SrRuO}_{3}$ films deposited on $\mathrm{TiO}_{2}$ terminated $\mathrm{SrTiO}_{3}$ substrates. A multilayer growth mode has been obtained for $\mathrm{PbTiO}_{3}$ films grown on $\mathrm{TiO}_{2}$ terminated

\footnotetext{
${ }^{11}$ Growth rate has been determined from the RHEED specular intensity oscillations.
} 

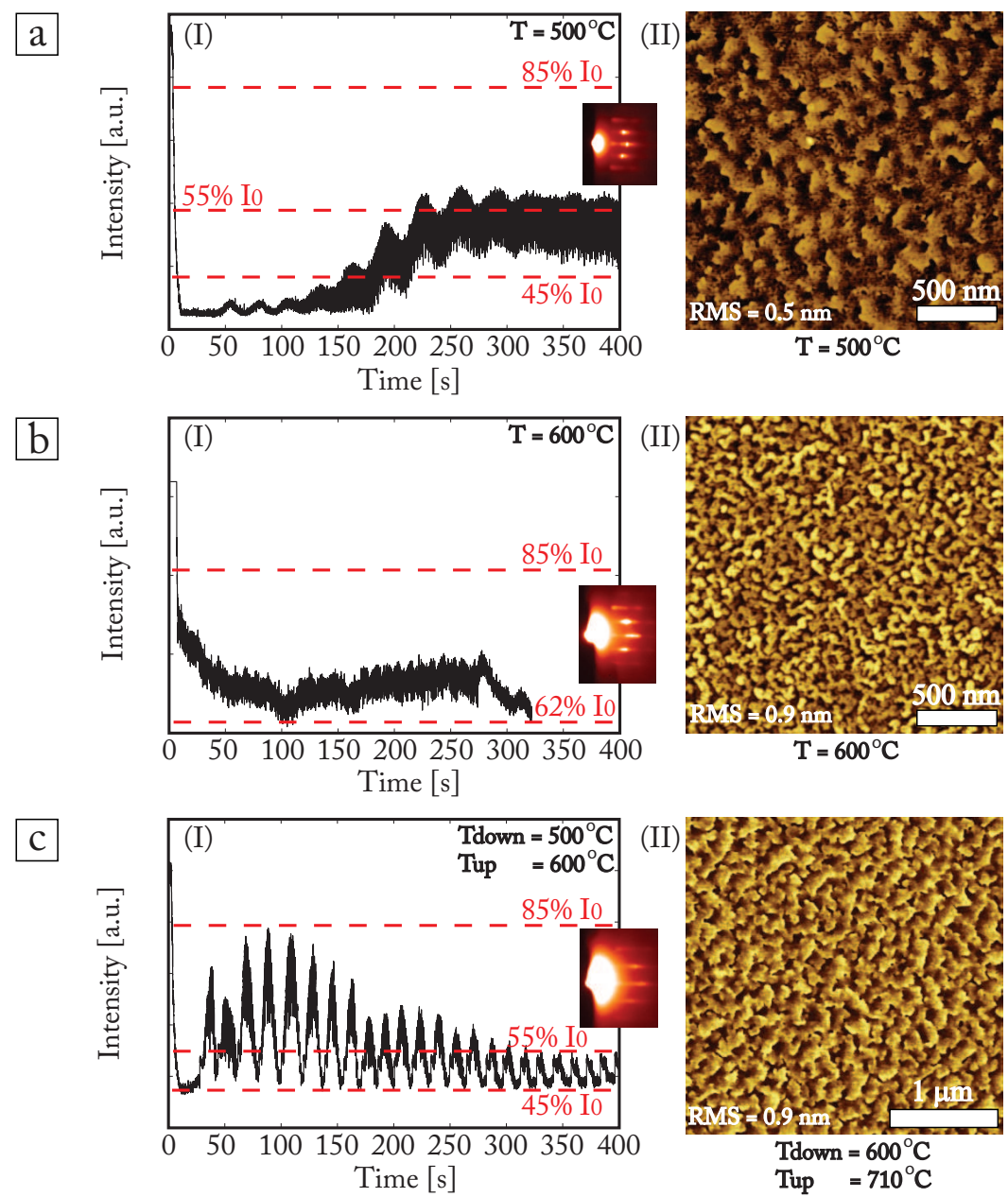

Fig. 6.9 (I) RHEED specular intensity during $\mathrm{PbTiO}_{3}$ growth and ex situ TM-AFM images after growth of $10 \mathrm{~nm} \mathrm{PbTiO}_{3}$ films a) $T=500{ }^{\circ} \mathrm{C}$, b) $T=600{ }^{\circ} \mathrm{C}$ and c) $T_{\text {down }}=500{ }^{\circ} \mathrm{C}, T_{\text {up }}=600{ }^{\circ} \mathrm{C}, n_{\text {down }}=0$ and $n_{\text {up }}=8$ (growth rate $=16$ pulses $\left./ \mathrm{ML}\right)$. Temperature sequence TM-PLD is started from the moment RHEED oscillations were observed.

$\mathrm{SrTiO}_{3}$ substrates, while $\mathrm{PbTiO}_{3}$ grows layer-by-layer on $\mathrm{SrRuO}_{3}$ up to $T=550$ $600{ }^{\circ} \mathrm{C}, 3 \mathrm{D}$ at $T=600-700{ }^{\circ} \mathrm{C}$ and layer-by-layer at $T=700{ }^{\circ} \mathrm{C}^{12}$. Fig. 6.9 shows the (I) RHEED specular intensity of $\mathrm{PbTiO}_{3}$ films deposited at $T=500{ }^{\circ} \mathrm{C}$, at $T=600{ }^{\circ} \mathrm{C}$ and at $T_{\text {down }}=500 / T_{\text {up }}=600{ }^{\circ} \mathrm{C}$, and (II) ex situ TM-AFM images recorded after $\mathrm{PbTiO}_{3}$ deposition are presented.

\footnotetext{
${ }^{12}$ After $\mathrm{PbTiO}_{3}$ deposition at $T=700{ }^{\circ} \mathrm{C}$, a $\mathrm{Pb}$ :Ti ratio $\approx 0$ has been found.
} 
At $T=500{ }^{\circ} \mathrm{C}$ and $T_{\text {down }}=500{ }^{\circ} \mathrm{C} / T_{\text {up }}=600{ }^{\circ} \mathrm{C}$, the first $1-2 \mathrm{PbTiO}_{3} \mathrm{ML}$ 's grow (s) in a multilayer fashion, whereafter the growth continues in a $2 \mathrm{D}$ growth mode (based on RHEED specular intensity). No RHEED oscillations have been observed for the $\mathrm{PbTiO}_{3}$ film growth at $T=600{ }^{\circ} \mathrm{C}$. From the RHEED pattern after growth, it is hardly distinguishable whether the growth was $2 \mathrm{D}$ or $3 \mathrm{D}$. During TM-PLD in Fig. 6.9 c), the maximum amplitude of the RHEED oscillation is $0.4 I_{0}$, which decreases to a constant amplitude of $0.1 I_{0}$. At $T=500{ }^{\circ} \mathrm{C}$, the amplitude of the RHEED specular intensity increases to a constant amplitude of $0.1 I_{0}$ after nine ML's. RMS values of $\sigma_{\mathrm{RMS}}=0.5 \mathrm{~nm}, \sigma_{\mathrm{RMS}}=0.9 \mathrm{~nm}$ and $\sigma_{\mathrm{RMS}}=0.9 \mathrm{~nm}$ have been extracted from AFM images for $T=500{ }^{\circ} \mathrm{C}, T=600{ }^{\circ} \mathrm{C}$ and $T_{\text {down }}=500{ }^{\circ} \mathrm{C}, T_{\text {up }}=600{ }^{\circ} \mathrm{C}$, respectively. The $\mathrm{PbTiO}_{3}$ of films grown in Figs. 6.9 a) and $6.9 \mathrm{c}$ ) contain surfaces where coalescence of islands did occur. This does not seem to be the case for the $\mathrm{PbTiO}_{3}$ surface grown at $T=600{ }^{\circ} \mathrm{C}$ as it has a fingerprint structure.

\subsubsection{Sticking of $\mathrm{Pb}$}

The atomic ratio between $\mathrm{Pb}$ :Ti has been measured using XPS for $\mathrm{PbTiO}_{3}$ films grown at different temperatures. The results are shown in Fig. 6.10. Eq. 6.4 has been used as fit based on an Arrhenius plot and the fit is represented by the (I) continuous (blue) line through the datapoints. Similar Pb:Ti ratio's have been found for $\mathrm{PbTiO}_{3}$ deposition at $T=450{ }^{\circ} \mathrm{C}, T=500{ }^{\circ} \mathrm{C}$ and $T=550{ }^{\circ} \mathrm{C}$. It seems that the $\mathrm{Pb}$ :Ti ratio starts to decrease in between deposition temperatures of $T=550-600{ }^{\circ} \mathrm{C}$. A significant lower $\mathrm{Pb}$ :Ti ratio has been measured after depositing $\mathrm{PbTiO}_{3}$ at $T=650{ }^{\circ} \mathrm{C}$. An $E_{\text {ads }}=0.8 \pm 0.1 \mathrm{eV}$ is determined based on the fit for deposition at (I) constant temperature ${ }^{13}$.

The (II) dashed line in Fig. 6.10 is an indicative fit for the TM-PLD samples. Datapoints are included representing $T_{\text {up }}=600{ }^{\circ} \mathrm{C}$ and $T_{\text {up }}=650{ }^{\circ} \mathrm{C}$, where $T_{\text {down }}=500{ }^{\circ} \mathrm{C}$. The samples have been grown such that temperature is decreased after maximum $I_{\text {RHEED }}$ and the temperature is increased directly after minimum $I_{\text {RHEED }}$. Both samples show an increase in $\mathrm{Pb}$ :Ti ratio when one compares these with samples grown at $T=T_{\text {up }}$. An $E_{\text {ads }}=1.6 \pm 0.1 \mathrm{eV}$ is used to fit the datapoints $^{14}$ of the (II) TM-PLD experiments.

\footnotetext{
${ }^{13}$ Assumption has been made that the sticking coefficient $\kappa_{\text {stick }}=1$ at $T=450-550{ }^{\circ} \mathrm{C}$.

14 Third datapoint used for the fit is the datapoint at $T=500{ }^{\circ} \mathrm{C}$.
} 

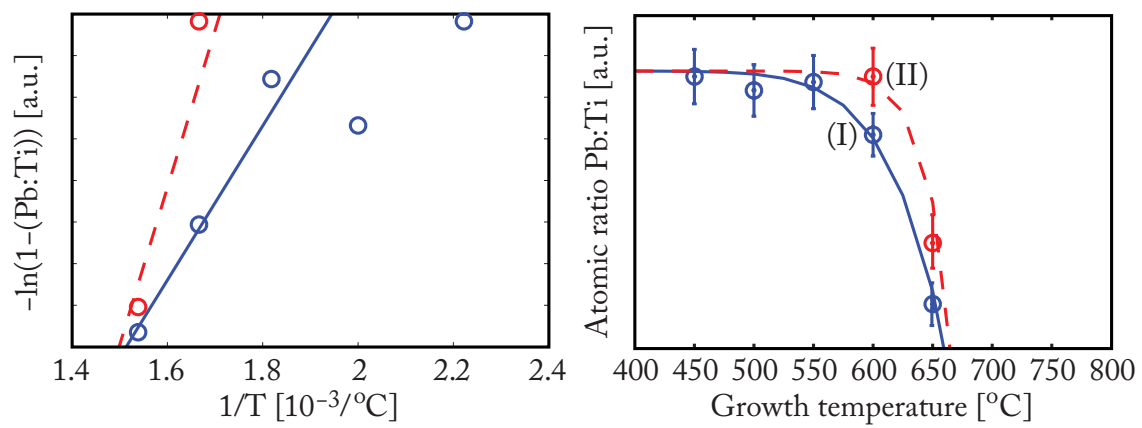

Fig. 6.10 The Arrhenius plot (left panel) and atomic ratio $\mathrm{Pb}$ :Ti (right panel) for $\mathrm{PbTiO}_{3}$ grown at different growth temperatures, (I) line - guide-to-the-eye fit for deposition at constant temperature and (II) dashed line expected fit for TM-PLD.

\subsection{Discussion}

In this section the KMC simulation and experimental results are discussed.

\section{Summary of results}

The results of KMC simulations and experiments described in this chapter indicate that:

- An increase in amplitude of RHEED specular intensity oscillations can be achieved during $2 \mathrm{D} \mathrm{SrTiO}_{3}$ homoepitaxial growth using TM-PLD predicted by KMC simulation and experimentally observed.

- An increase in amplitude of RHEED specular intensity oscillations can be obtained by depositing the first ML growth stage at lower $T$ and the second ML growth stage at higher $T$.

- An instantaneous temperature increase results in an increase in amplitude of RHEED specular intensity oscillations (compared to a more realistic ramprate $\left.=20^{\circ} \mathrm{C} / \mathrm{s}\right)$.

- Including a ramp-rate $\left(20^{\circ} \mathrm{C} / \mathrm{s}\right)$ instead of an instantaneous temperature increase results in a decrease of the pulse, whereafter the temperature is increased in order to achieve a maximum recovery of RHEED specular intensity. 
- The Pb:Ti ratio in $\mathrm{PbTiO}_{3}$ starts to decrease in between $T=550-600{ }^{\circ} \mathrm{C}$. An $E_{\text {ads }}=0.8 \pm 0.1 \mathrm{eV}$ has been found for $\mathrm{Pb}$. The $\mathrm{Pb}$ :Ti ratio can be increased by TM-PLD.

By introducing two temperatures, a ramp-rate and the number of pulses whereafter the temperature needs to increase and decrease again, the influence of temperature cycles during PLD can be studied using an adapted solid-on-solid model. The KMC simulation results show a qualitative agreement with experimental data obtained for $\mathrm{SrTiO}_{3}$ homoepitaxial growth using $E_{\mathrm{D}}=2.2 \mathrm{eV}$ and $E_{\mathrm{N}}=0.27 \mathrm{eV}$. A previous study used these values and a qualitative agreement between KMC simulation and experiment has been shown [58]. Many other studies proved this qualitative agreement for PLD growth of other perovskite oxides [18, 148]. It is unknown, how TM-PLD KMC simulation results will change using an different $E_{\mathrm{N}}=0.21 \pm 0.01 \mathrm{eV}$ determined from AFM experiments described in Ch. 5 .

From Ch. 2 (Eq. 2.5), it is known that $I_{\text {RHEED }}$ scales with the step density $S$. As the amplitude of $I_{\text {RHEED }}$ oscillations will increase using the described sequence, it seems plausible that the difference between the minimum and maximum $S$ will become larger using TM-PLD [18]. The larger difference in $S$ is explained by the fact that $S$ increases at lower $T$ and thereafter the increase in $T$ enhances the diffusion length $l_{\mathrm{D}}$ /interlayer mass transport of species at the second part of a ML. An increase in interlayer mass transport at the final part of a ML results in an stronger recovery of $I_{\text {RHEED }}$ indicating a reduced $S$ and smoother surface topography. A similar approach and explanation has been reported using flux modulation [44]. In the reversed sequence, the amplitude of $I_{\text {RHEED }}$ are small and at the minimum $I_{\text {RHEED }}$ a much lower $S$ is obtained explained by the deposition at higher $T$ and enhanced $l_{\mathrm{D}}$.

The different results between using a (more realistic) ramp-rate or instantaneous increase/decrease in temperature is explained by the time it takes to increase $l_{\mathrm{D}}$. An exponential scaling between the diffusion coefficient $D_{\mathrm{S}}$ and $T$ has been reported, see Ch. 2 (Eq. 2.2) [148]. Using an instantaneous increase/decrease in temperature, most of the interlayer mass transport seem to occur within a single second, while it already takes five seconds to overcome a temperature difference of $100{ }^{\circ} \mathrm{C}\left(T_{\text {up }}-T_{\text {down }}\right)$ using a ramp-rate of $20{ }^{\circ} \mathrm{C} / \mathrm{s}$. In order to achieve the same diffusion length $l_{\mathrm{D}}$ in the last second in a ML deposition, the ramp-rate has to start at least five seconds before. An increase in amplitude of $I_{\text {RHEED }}$ oscillations can be achieved by using a laser heater containing a ramp-rate $\geq 20{ }^{\circ} \mathrm{C} / \mathrm{s}$.

The formula describing the sticking of species fits the $\mathrm{Pb}$ :Ti atomic ratio measurements. Previously, fitting of the atomic ratio's $\mathrm{Bi} / \mathrm{Y}$ and $\mathrm{Bi} / \mathrm{Ba}$ have been performed in the same manner for $\mathrm{YBiO}_{3}$ and $\mathrm{BaBiO}_{3}$, where $\mathrm{Bi}$ is the volatile 
element [242]. The experiments reveal that directly growing $\mathrm{PbTiO}_{3}$ films on $\mathrm{TiO}_{2}$ terminated $\mathrm{SrTiO}_{3}$ results in multilayer growth, while on $\mathrm{SrRuO}_{3}$ it grows in a $2 \mathrm{D}$ fashion. Similar PLD growth behavior have been reported for $\mathrm{BiFeO}_{3}$, where $\mathrm{Bi}$ is volatile and the $\mathrm{A}$ cation in $\mathrm{ABO}_{3}$ ( $\mathrm{Pb}$ is $\mathrm{A}$ cation in $\left.\mathrm{PbTiO}_{3}\right)$ [191]. During the growth of the first few ML's, no RHEED specular intensity oscillations are observed, where from the third ML RHEED oscillations appear. KMC simulations need to be repeated in order to know how TM-PLD influences the $\mathrm{PbTiO}_{3}$ growth as a different value of $E_{\mathrm{D}}$ for $\mathrm{PbTiO}_{3}$ is expected. Other perovskite oxides containing volatile species have been considered, such as $\mathrm{SrRuO}_{3}$ ( $\mathrm{Ru}$ is volatile) and $\mathrm{BiFeO}_{3}$. However, these materials show transitions in growth mode or prefer to grow $1 \mathrm{D}$ or in multilevel fashion (2D growth is preferred as RHEED oscillations can be used as an indication when the temperature needs to be increased/decreased) [8, 191].

Despite the fact that TM-PLD $\mathrm{PbTiO}_{3}$ films show a higher $\mathrm{Pb}$ :Ti ratio compared to $\mathrm{PbTiO}_{3}$ films grown at $T=T_{\text {up }}$, the direct relation between an increase in $S$ and increase in $\kappa_{\text {stick }}$ was not proven yet. For example, when a $\mathrm{PbTiO}_{3}$ film is grown alternating $T_{\text {down }}$ during odd $\mathrm{ML}$ deposition $(\mathrm{ML}=1,3,5 \ldots$ etc grown at $\left.T_{\text {down }}\right)$ and $T_{\text {up }}$ during even ML deposition ( $\mathrm{ML}=2,4,6 \ldots$ etc grown at $\left.T_{\text {up }}\right)$, does this result in a similar Pb:Ti atomic ratio? How does TM-PLD affect the crystallization and properties of $\mathrm{PbTiO}_{3}$ films? Follow-up investigations, both simulations and experiments, are needed to answer these research questions.

\subsection{Conclusions \& outlook}

The influence of TM-PLD on the step density during growth is explored in order to increase sticking of volatile species in perovskite oxides. KMC simulations and $\mathrm{SrTiO}_{3}$ homoepitaxial growth experiments reveal that the amplitude of RHEED specular intensity oscillations can be increased using TM-PLD. Therefore, an increase in the difference between the minimum and maximum step density in a ML can be achieved compared to PLD at constant temperature. Atomic ratio measurements show that the $\mathrm{Pb}$ :Ti ratio in $\mathrm{PbTiO}_{3}$ starts to decrease between $T=550-600{ }^{\circ} \mathrm{C}$. Using TM-PLD, an increase in Pb:Ti ratio has been obtained when comparing it with samples grown at $T=T_{\text {up }} \geq 600{ }^{\circ} \mathrm{C}$. In that case, the $\mathrm{Pb}$ :Ti ratio is increased. Nevertheless, a relation between an increase in maximum step density and an increase sticking coefficient was not proven yet. 
The following experiments are suggested to validate whether an increase in maximum step density causes an increase in sticking coefficient:

- A different $\mathrm{Pb}$ :Ti should be obtained when depositing a $\mathrm{PbTiO}_{3}$ film at the same $T_{\text {down }}$ and $T_{\text {up }}$, but using a different $n_{\text {down }}$ and $n_{\text {up }}$.

- Pulsed laser interval deposition (PLID) instead of TM-PLD to show increase of $\mathrm{PbTiO}_{3}$ sticking coefficient compared to deposition at constant temperature [44].

Besides that, the experimental RHEED specular intensity scales with the step density, it has a dependence on the crystalline state as well. This dependence makes it more complicated to draw conclusions regarding to KMC simulations. Direct step density observations with (real-time) in situ AFM can overcome the addressed issue. So far, in situ AFM has not been used as the heater described in Ch. 3 is not capable of heating and cooling rates used in these experiments. Moreover, the in situ AFM needs to be designed such that it operates more stable when the temperature of the instrument starts to change. 



\section{Bibliography}

[1] Oxford dictionaries.

[2] J. A. Venables and G. D. T. Spiller. Surface mobilities on solid materials: fundamental concepts and applications 341. Springer US Boston, MA (1983).

[3] R. Eason. Pulsed laser deposition of thin films: applications-led growth of functional materials. (2009).

[4] G. Rijnders and D. H. A. Blank. Materials science: Build your own superlattice. Nature 433, 369 (2005).

[5] J. G. Bednorz and K. A. Muller. Possible high-Tc superconductivity in the BaLaCuO system. Zeitschrift fur Physik B Condensed Matter 64, 189 (1986).

[6] D. Dijkkamp, T. Venkatesan, X. D. Wu, S. A. Shaheen, N. Jisrawi, Y. H. Min-Lee, W. L. McLean, and M. Croft. Preparation of $Y$-Ba-Cu oxide superconductor thin films using pulsed laser evaporation from high-Tc bulk material. Applied Physics Letters 51, 619 (1987).

[7] D. H. A. Blank, M. Dekkers, and G. Rijnders. Pulsed laser deposition in Twente: from research tool towards industrial deposition. Journal of Physics D: Applied Physics 47, 034006 (2014).

[8] G. Rijnders, D. H. A. Blank, J. Choi, and C. B. Eom. Enhanced surface diffusion through termination conversion during epitaxial SrRuO $\mathrm{O}_{3}$ growth. Applied Physics Letters 84, 505 (2004).

[9] I. Bozovic and J. Eckstein. Analysis of growing films of complex oxides by RHEED. MRS Bulletin 20, 32 (1995).

[10] H. Karl and B. Stritzker. Reflection high-energy electron diffraction oscillations modulated by laser-pulse deposited YBCO. Phys. Rev. Lett. 69, 2939 (1992).

[11] M. Yoshimoto, H. Ohkubo, N. Kanda, H. Koinuma, K. Horiguchi, M. Kumagai, and K. Hirai. Atomic force microscopy on homoepitaxial $\mathrm{SrTiO}_{3}$ films grown under monitoring of intensity oscillation in reflection high energy electron diffraction. Applied Physics Letters 61, 2659 (1992).

[12] Z. Liu, T. Hanada, R. Sekine, M. Kawai, and H. Koinuma. Atomic layer control in Sr-Cu-O artificial lattice growth. Applied Physics Letters 65, 1717 (1994). 
[13] M. Kanai, T. Kawai, and S. Kawai. Atomic layer and unit cell layer growth of ( $\mathrm{Ca}, \mathrm{Sr}) \mathrm{CuO}_{2}$ thin film by laser molecular beam epitaxy. Applied Physics Letters 58, 771 (1991).

[14] T. Terashima, Y. Bando, K. Iijima, K. Yamamoto, K. Hirata, K. Hayashi, K. Kamigaki, and H. Terauchi. Reflection high-energy electron diffraction oscillations during epitaxial growth of high-temperature superconducting oxides. Physical Review Letters 65, 2684 (1990).

[15] T. Shaw, A. Gupta, M. Chern, P. Batson, R. Laibowitz, and B. Scott. Atomic scale oxide superlattices grown by RHEED controlled pulsed laser deposition. Journal of Materials Research 9, 2566 (1994).

[16] G. J. H. M. Rijnders, G. Koster, D. H. A. Blank, and H. Rogalla. In situ monitoring during pulsed laser deposition of complex oxides using reflection high energy electron diffraction under high oxygen pressure. Applied Physics Letters 70, 1888 (1997).

[17] G. Koster. Artificially layered oxides by pulsed laser deposition. PhD thesis University of Twente Enschede, The Netherlands (September 1999).

[18] A. J. H. M. Rijnders. The initial growth of complex oxides: study and manipulation. PhD thesis University of Twente Enschede, The Netherlands (October 2001).

[19] G. Koster, B. L. Kropman, G. J. H. M. Rijnders, D. H. A. Blank, and H. Rogalla. Quasiideal strontium titanate crystal surfaces through formation of strontium hydroxide. Applied Physics Letters 73, 2920 (1998).

[20] J. E. Kleibeuker, G. Koster, W. Siemons, D. Dubbink, B. Kuiper, J. L. Blok, C. H. Yang, J. Ravichandran, R. Ramesh, J. E. ten Elshof, D. H. A. Blank, and G. Rijnders. Atomically Defined Rare-Earth Scandate Crystal Surfaces. Advanced Functional Materials 20, 3490 (2010).

[21] H. Lee, H. Christen, M. Chisholm, C. Rouleau, and D. H. Lowndes. Strong polarization enhancement in asymmetric three-component ferroelectric superlattices. Nature 433, 395 (2005).

[22] H. Y. Ohtomo, A. \& Hwang. A high-mobility electron gas at the $\mathrm{LaAlO}_{3} / \mathrm{SrTiO}_{3}$ heterointerface. Nature 427, 423 (2004).

[23] J. A. Stroscio, D. T. Pierce, and R. A. Dragoset. Homoepitaxial growth of iron and a real space view of reflection-high-energy-electron diffraction. Physical Review Letters 70, 3615 (1993).

[24] K. Morgenstern. Fast scanning tunnelling microscopy as a tool to understand changes on metal surfaces: from nanostructures to single atoms. Physica Status Solidi B: Basic Solid State Physics 242, 773 (2005).

[25] M. J. Rost. In situ real-time observation of thin film deposition: roughening, zeno effect, grain boundary crossing barrier, and steering. Physical Review Letters 99, 266101 (2007).

[26] M. Lippmaa, M. Kawasaki, A. Ohtomo, T. Sato, M. Iwatsuki, and H. Koinuma. Observation of $\mathrm{SrTiO}_{3}$ step edge dynamics by real-time high-temperature STM. Applied Surface Science 130, 582 (1998). 
[27] M. Lippmaa, N. Nakagawa, T. Kinoshita, T. Furumochi, M. Kawasaki, and H. Koinuma. Growth dynamics of oxide thin films at temperatures above $1000^{\circ} \mathrm{C}$. Physica C Superconductivity 335, 196 (2000).

[28] K. Iwaya, T. Ohsawa, R. Shimizu, T. Hashizume, and T. Hitosugi. Atomically resolved surface structure of $\mathrm{SrTiO}_{3}(001)$ thin films grown in step-flow mode by pulsed laser deposition. Applied Physics Express 3, 075701 (2010).

[29] R. Shimizu, T. Ohsawa, K. Iwaya, S. Shiraki, and T. Hitosugi. Epitaxial growth process of $\mathrm{La}_{0.7} \mathrm{Ca}_{0.3} \mathrm{MnO}_{3}$ thin films on $\mathrm{SrTiO}_{3}(001)$ : thickness-dependent inhomogeneity caused by excess Ti atoms. Crystal Growth \& Design 14, 1555 (2014).

[30] G. Binnig, C. F. Quate, and C. Gerber. Atomic force microscope. Physical Review Letters 56, 930 (1986).

[31] G. Binnig, H. Rohrer, C. Gerber, and E. Weibel. Surface studies by scanning tunneling microscopy. Physical Review Letters 49, 57 (1982).

[32] L. Sudheendra, V. Moshnyaga, E. D. Mishina, B. Damaschke, T. Rasing, and K. Samwer. Direct imaging of lattice-strain-induced stripe phases in an optimally doped manganite film. Physical Review B: Condensed Matter and Materials Physics 75, 172407 (2007).

[33] J. J. Broekmaat. In situ growth monitoring with scanning force microscopy during pulsed laser deposition. PhD thesis University of Twente Enschede, The Netherlands (April 2008).

[34] J. Broekmaat, A. Brinkman, D. H. A. Blank, and G. Rijnders. High temperature surface imaging using atomic force microscopy. Applied Physics Letters 92, 043102 (2008).

[35] M. J. Rost, L. Crama, P. Schakel, E. van Tol, G. B. E. M. van Velzen-Williams, C. F. Overgauw, H. ter Horst, H. Dekker, B. Okhuijsen, M. Seynen, A. Vijftigschild, P. Han, A. J. Katan, K. Schoots, R. Schumm, W. van Loo, T. H. Oosterkamp, and J. W. M. Frenken. Scanning probe microscopes go video rate and beyond. Review of Scientific Instruments 76, 053710 (2005).

[36] G. E. Fantner, G. Schitter, J. H. Kindt, T. Ivanov, K. Ivanova, R. Patel, N. HoltenAndersen, J. Adams, P. J. Thurner, I. W. Rangelow, and P. K. Hansma. Components for high speed atomic force microscopy. Ultramicroscopy 106, 881 (2006).

[37] L. M. Picco, L. Bozec, A. Ulcinas, D. J. Engledew, M. Antognozzi, M. A. Horton, and M. J. Miles. Breaking the speed limit with atomic force microscopy. Nanotechnology 18, 044030 (2007).

[38] G. Schitter and M. J. Rost. Scanning probe microscopy at video-rate. Materials Today 11, 40 (2008).

[39] T. Ando, T. Uchihashi, and T. Fukuma. High-speed atomic force microscopy for nanovisualization of dynamic biomolecular processes. Progress in Surface Science 83, 337 (2008).

[40] Y. K. Yong, S. O. R. Moheimani, B. J. Kenton, and K. K. Leang. High-speed flexure-guided nanopositioning: mechanical design and control issues. Review of Scientific Instruments 83, 121101 (2012).

[41] J. W. Reiner, F. J. Walker, and C. H. Ahn. Atomically engineered oxide interfaces. Science 323, 1018 (2009). 
[42] D. G. Schlom, L. Q. Chen, X. Pan, A. Schmehl, and M. A. Zurbuchen. A thin film approach to engineering functionality into oxides. Journal of the American Ceramic Society 91, 2429 (2008).

[43] R. Ramesh, K. Luther, B. Wilkens, D. L. Hart, E. Wang, J. M. Tarascon, A. Inam, X. D. $\mathrm{Wu}$, and T. Venkatesan. Epitaxial growth of ferroelectric bismuth titanate thin films by pulsed laser deposition. Applied Physics Letters 57, 1505 (1990).

[44] G. Koster, G. J. H. M. Rijnders, D. H. A. Blank, and H. Rogalla. Imposed layer-by-layer growth by pulsed laser interval deposition. Applied Physics Letters 74, 3729 (1999).

[45] A. Borowiak, G. Niu, V. Pillard, G. Agnus, P. Lecoeur, D. Albertini, N. Baboux, B. Gautier, and B. Vilquin. Pulsed laser deposition of epitaxial ferroelectric $\mathrm{Pb}(\mathrm{Zr}, \mathrm{Ti}) \mathrm{O}_{3}$ films on silicon substrates. Thin Solid Films 520, 4604 (2012).

[46] R. J. Spah, H. F. Hess, H. L. Stormer, A. E. White, and K. T. Short. Parameters for in situ growth of high Tc superconducting thin films using an oxygen plasma source. Applied Physics Letters 53, 441 (1988).

[47] D. G. Schlom, J. N. Eckstein, E. S. Hellman, S. K. Streiffer, J. S. Harris, M. R. Beasley, J. C. Bravman, T. H. Geballe, C. Webb, K. E. von Dessonneck, and F. Turner. Molecular beam epitaxy of layered Dy-Ba-Cu-O compounds. Applied Physics Letters 53, 1660 (1988).

[48] J. N. Eckstein, , and I. Bozovic. High-temperature superconducting multilayers and heterostructures grown by atomic layer-by-layer molecular beam epitaxy. Annual Review of Materials Science 25, 679 (1995).

[49] C. Theis, J. Yeh, D. Schlom, M. Hawley, and G. Brown. Adsorption-controlled growth of $\mathrm{PbTiO}_{3}$ by reactive molecular beam epitaxy. Thin Solid Films 325, 107 (1998).

[50] U. Poppe, J. Schubert, R. Arons, W. Evers, C. Freiburg, W. Reichert, K. Schmidt, W. Sybertz, and K. Urban. Direct production of crystalline superconducting thin films of $\mathrm{YBa}_{2} \mathrm{Cu}_{3} \mathrm{O}_{7}$ by high-pressure oxygen sputtering. Solid State Communications 66, 661 (1988).

[51] P. Muralt, T. Maeder, L. Sagalowicz, S. Hiboux, S. Scalese, D. Naumovic, R. G. Agostino, N. Xanthopoulos, H. J. Mathieu, L. Patthey, and E. L. Bullock. Texture control of PbTiO and $\mathrm{Pb}(\mathrm{Zr}, \mathrm{Ti}) \mathrm{O}_{3}$ thin films with $\mathrm{TiO}_{2}$ seeding. Journal of Applied Physics 83, 3835 (1998).

[52] N. K. Pervez, P. J. Hansen, and R. A. York. High tunability barium strontium titanate thin films for rf circuit applications. Applied Physics Letters 85, 4451 (2004).

[53] H. Koinuma, M. Kawasaki, M. Funabashi, T. Hasegawa, K. Kishio, K. Kitazawa, K. Fueki, and S. Nagata. Preparation of superconducting thin films of $\left(\mathrm{La}_{1-x} \mathrm{Sr}_{x}\right)_{y} \mathrm{CuO} \mathrm{O}_{4}$ by sputtering. Journal of Applied Physics 62, 1524 (1987).

[54] J. Triscone, L. Frauchiger, M. Decroux, L. Miéville, O. Fischer, C. Beeli, P. Stadelmann, and G. A. Racine. Growth and structural properties of epitaxial $\mathrm{Pb}\left(Z r_{x} T i_{1-x}\right) \mathrm{O}_{3}$ films and $\mathrm{Pb}\left(\mathrm{Zr}_{x} \mathrm{Ti}_{1-x}\right) \mathrm{O}_{3}$ cuprate heterostructures. Journal of Applied Physics 79, 4298 (1996).

[55] S. D. Bu, M. K. Lee, C. B. Eom, W. Tian, X. Q. Pan, S. K. Streiffer, and J. J. Krajewski. Perovskite phase stabilization in epitaxial $\mathrm{Pb}\left(\mathrm{Mg}_{1 / 3} \mathrm{Nb}_{2 / 3}\right) \mathrm{O}_{3}-\mathrm{PbTiO}_{3}$ films by deposition onto vicinal (001) $\mathrm{SrTiO}_{3}$ substrates. Applied Physics Letters 79, 3482 (2001). 
[56] J. Neave, B. Joyce, P. Dobson, and N. Norton. Dynamics of film growth of GaAs by $M B E$ from RHEED observations. Applied Physics A: Materials Science \& Processing 31, 1 (1983).

[57] T. Shitara, D. D. Vvedensky, M. R. Wilby, J. Zhang, J. H. Neave, and B. A. Joyce. Morphological model of reflection high-energy electron diffraction intensity oscillations during epitaxial growth on GaAs(001). Applied Physics Letters 60, 1504 (1992).

[58] D. H. A. Blank, G. Koster, G. Rijnders, E. van Setten, P. Slycke, and H. Rogalla. Imposed layer-by-layer growth by pulsed laser interval deposition. Applied physics A: Materials science \& processing 69, S17 (1999).

[59] G. Eres, J. Z. Tischler, M. Yoon, B. C. Larson, C. M. Rouleau, D. H. Lowndes, and P. Zschack. Time-resolved study of $\mathrm{SrTiO}_{3}$ homoepitaxial pulsed-laser deposition using surface $x$-ray diffraction. Applied Physics Letters 80, 3379 (2002).

[60] J. H. Lee, I. C. Tung, S.-H. Chang, A. Bhattacharya, D. D. Fong, J. W. Freeland, and H. Hong. In situ surface/interface $x$-ray diffractometer for oxide molecular beam epitaxy. Review of Scientific Instruments 87, 013901 (2016).

[61] M. J. Rost, G. J. C. van Baarle, A. J. Katan, W. M. van Spengen, P. Schakel, W. A. van Loo, T. H. Oosterkamp, and J. W. M. Frenken. Video-rate scanning probe control challenges: setting the stage for a microscopy revolution. Asian Journal of Control 11, 110 (2009).

[62] T. Ando. High-speed atomic force microscopy coming of age. Nanotechnology 23, 062001 (2012).

[63] T. Ando. High-speed atomic force microscopy. Microscopy 62, 81 (2013).

[64] B. P. Brown, L. Picco, M. J. Miles, and C. F. J. Faul. Opportunities in high-speed atomic force microscopy. Small 9, 3201 (2013).

[65] B. Schlecker, M. Dukic, B. Erickson, M. Ortmanns, G. Fantner, and J. Anders. Singlecycle-PLL detection for real-time FM-AFM applications. IEEE Transactions on Biomedical Circuits and Systems 8, 206 (2014).

[66] J. Adams, B. Erickson, J. Grossenbacher, J.and Brugger, A. Nievergelt, and G. Fantner. Harnessing the damping properties of materials for high-speed atomic force microscopy. Nature Nanotechnology 11, 147 (2015).

[67] V. E. Henrich and P. A. Cox. The Surface Science of Metal Oxides. Cambridge University Press (1996).

[68] S. E. Park and T. R. Shrout. Ultrahigh strain and piezoelectric behavior in relaxor based ferroelectric single crystals. Journal of Applied Physics 82, 1804 (1997).

[69] I. Vrejoiu, G. Le Rhun, L. Pintilie, D. Hesse, M. Alexe, and U. Gösele. Intrinsic ferroelectric properties of strained tetragonal $\mathrm{PbZr}_{0.2} \mathrm{Ti}_{0.8} \mathrm{O}_{3}$ obtained on layer-by-layer grown, defectfree single-crystalline films. Advanced Materials 18, 1657 (2006).

[70] B. T. Matthias, R. M. Bozorth, and J. H. Van Vleck. Ferromagnetic interaction in EuO. Physical Review Letters 7, 160 (1961). 
[71] A. Maignan, C. Simon, V. Caignaert, and B. Raveau. Giant magnetoresistance ratios superior to 1011 in manganese perovskites. Solid State Communications 96, 623 (1995).

[72] G. Petrich, S. von Molnár, and T. Penney. Exchange-induced autoionization in Eu-rich EuO. Physical Review Letters 26, 885 (1971).

[73] R. J. Soulen, J. M. Byers, M. S. Osofsky, B. Nadgorny, T. Ambrose, S. F. Cheng, P. R. Broussard, C. T. Tanaka, J. Nowak, J. S. Moodera, A. Barry, and J. M. D. Coey. Measuring the spin polarization of a metal with a superconducting point contact. Science 282, 85 (1998).

[74] Crystec GmbH.

[75] S. S. A. Seo and H. N. Lee. Strain-coupled ferroelectric polarization in $\mathrm{BaTiO}_{3}-\mathrm{CaTiO}_{3}$ superlattices. Applied Physics Letters 94, 232904 (2009).

[76] F. García-Alvarado, E. Morán, and M. Alario-Franco. The modulation of the monoclinic $\mathrm{Bi}_{2} \mathrm{Sr}_{2} \mathrm{CuO}_{6+x}$ phase. Journal of Solid State Chemistry 98, 245 (1992).

[77] X. Q. Pan, J. C. Jiang, C. D. Theis, and D. G. Schlom. Domain structure of epitaxial $\mathrm{Bi}_{4} \mathrm{Ti}_{3} \mathrm{O}_{12}$ thin films grown on (001) $\mathrm{SrTiO}_{3}$ substrates. Applied Physics Letters 83, 2315 (2003).

[78] A. W. McConnell, R. A. Hughes, A. Dabkowski, H. A. Dabkowska, J. S. Preston, J. E. Greedan, and T. Timusk. Evaluation of $\mathrm{LaSrGaO}_{4}$ as a substrate for $\mathrm{YBa}_{2} \mathrm{Cu}_{3} \mathrm{O}_{7-x}$. Physica C Superconductivity 225, 7 (1994).

[79] L. Ceresara, F. Fuso, E. Arimondo, and P. Scardi. Pulsed laser deposition of YBCO thin films on metal substrates with YSZ buffer layer. Il Nuovo Cimento D 19, 1033 (1997).

[80] R. Nechache, C. Harnagea, L. Gunawan, L. p. Carignan, C. Maunders, D. Menard, G. A. Botton, and A. Pignolet. Growth, structure, and properties of $\mathrm{BiFeO}_{3}-\mathrm{BiCrO}_{3}$ films obtained by dual cross beam PLD. IEEE Transactions on Ultrasonics, Ferroelectrics, and Frequency Control 54, 2645 (2007).

[81] Y. Weng, X. Huang, Y. Tang, and S. Dong. Magnetic orders of LaTiO 3 under epitaxial strain: a first-principles study. Journal of Applied Physics 115, 17E108 (2014).

[82] J. H. Lee, L. Fang, E. Vlahos, X. Ke, Y. W. Jung, L. F. Kourkoutis, J.-W. Kim, P. J. Ryan, T. Heeg, M. Roeckerath, et al. A strong ferroelectric ferromagnet created by means of spin-lattice coupling. Nature 466, 954 (2010).

[83] Y. Shimakawa, Y. Kubo, Y. Nakagawa, S. Goto, T. Kamiyama, H. Asano, and F. Izumi. Crystal structure and ferroelectric properties of $A \mathrm{Bi}_{2} \mathrm{Ta}_{2} \mathrm{O}_{9}(A=\mathrm{Ca}, S r$, and $B a)$. Physical Review B: Condensed Matter and Materials Physics 61, 6559 (2000).

[84] MTI Corporation.

[85] F. Johann, A. Morelli, D. Biggemann, M. Arredondo, and I. Vrejoiu. Epitaxial strain and electric boundary condition effects on the structural and ferroelectric properties of $\mathrm{BiFeO}_{3}$ films. Physical Review B: Condensed Matter and Materials Physics 84, 094105 (2011).

[86] H. M. Christen, G. E. Jellison, I. Ohkubo, S. Huang, M. E. Reeves, E. Cicerrella, J. L. Freeouf, Y. Jia, and D. G. Schlom. Dielectric and optical properties of epitaxial rare-earth scandate films and their crystallization behavior. Applied Physics Letters 88, 262906 (2006). 
[87] X. Fan, W. Zheng, X. Chen, and D. J. Singh. 2DEGs at perovskite interfaces between $\mathrm{KTaO}_{3}$ or $\mathrm{KNbO}_{3}$ and stannates. PLOS ONE 9, e91423 (2014).

[88] B. Noheda, D. E. Cox, G. Shirane, J. A. Gonzalo, L. E. Cross, and S.-E. Park. A monoclinic ferroelectric phase in the $\mathrm{Pb}\left(Z r_{1-x} T i_{x}\right) \mathrm{O}_{3}$ solid solution. Applied Physics Letters 74, 2059 (1999).

[89] S. Trolier-McKinstry, M. D. Biegalski, J. Wang, A. A. Belik, E. Takayama-Muromachi, and I. Levin. Growth, crystal structure, and properties of epitaxial $\mathrm{BiScO}_{3}$ thin films. Journal of Applied Physics 104, 044102 (2008).

[90] G. Li, B. Yan, R. Thomale, and W. Hanke. Topological nature and the multiple Dirac cones hidden in Bismuth high-Tc superconductors. Scientific Reports 5, 10435 (2015).

[91] M. E. Klausmeier Brown, J. N. Eckstein, I. Bozovic, and G. F. Virshup. Accurate measurement of atomic beam flux by pseudo double beam atomic absorption spectroscopy for growth of thin film oxide superconductors. Applied Physics Letters 60, 657 (1992).

[92] A. Cho and J. Arthur. Molecular beam epitaxy. Progress in Solid State Chemistry 10, 157 (1975).

[93] D. G. Schlom. Perspective: Oxide molecular-beam epitaxy rocks! APL Materials 3, 062403 (2015).

[94] R. A. Betts and C. W. Pitt. Growth of thin-film lithium niobate by molecular beam epitaxy. Electronics Letters 21, 960 (1985).

[95] J. Kwo, M. Hong, D. J. Trevor, R. M. Fleming, A. E. White, R. C. Farrow, A. R. Kortan, and K. T. Short. In situ epitaxial growth of YBCO films by molecular beam epitaxy with an activated oxygen source. Applied Physics Letters 53, 2683 (1988).

[96] H. Nonaka, T. Shimizu, and K. Arai. Preparation of NBCO films in ultrahigh vacuum with a $\mathrm{NO}_{2}$ supersonic molecular beam. Applied Physics Letters 57, 2850 (1990).

[97] V. Achutharaman, K. Beauchamp, N. Chandrasekhar, G. Spalding, B. Johnson, and A. Goldman. Fabrication of high-Tc superconductors using ozone-assisted molecular beam epitaxy. Thin Solid Films 216, 14 (1992).

[98] J. P. Locquet, C. Gerber, A. Cretton, Y. Jaccard, E. Williams, and E. Mächler. Electrochemical oxidation of $\mathrm{La}_{2} \mathrm{CuO}_{4}$ thin films grown by molecular beam epitaxy. Applied Physics A: Materials Science \& Processing 57, 211 (1993).

[99] R. A. McKee, F. J. Walker, J. R. Conner, E. D. Specht, and D. E. Zelmon. Molecular beam epitaxy growth of epitaxial barium silicide, barium oxide, and barium titanate on silicon. Applied Physics Letters 59, 782 (1991).

[100] T. Tsurumi, T. Suzuki, M. Yamane, and M. Daimon. Fabrication of barium titanate/strontium titanate artificial superlattice by atomic layer epitaxy. Japanese Journal of Applied Physics 33, 5192 (1994).

[101] R. A. McKee, F. J. Walker, and M. F. Chisholm. Crystalline oxides on silicon: the first five monolayers. Physical Review Letters 81, 3014 (1998). 
[102] C. D. Theis, J. Yeh, D. G. Schlom, M. E. Hawley, G. W. Brown, J. C. Jiang, and X. Q. Pan. Adsorption-controlled growth of $\mathrm{Bi}_{4} \mathrm{Ti}_{3} \mathrm{O}_{12}$ by reactive $\mathrm{MBE}$. Applied Physics Letters $\mathbf{7 2}$, 2817 (1998).

[103] S. Migita, H. Ota, H. Fujino, Y. Kasai, and S. Sakai. Epitaxial Bi ${ }_{4} \mathrm{Ti}_{3} \mathrm{O}_{12}$ thin film growth using Bi self-limiting function. Journal of Crystal Growth 200, 161 (1999).

[104] G. M. Roesler, Jr., M. E. Filipkowski, P. R. Broussard, Y. U. Idzerda, M. S. Osofsky, and R. J. Soulen, Jr. Epitaxial multilayers of ferromagnetic insulators with nonmagnetic metals and superconductors. Proc. SPIE 2157, 285 (1994).

[105] N. Iwata, G. Pindoria, T. Morishita, and K. Kohn. Preparation and magnetic properties of $\mathrm{EuO}$ thin films epitaxially grown on $\mathrm{MgO}$ and $\mathrm{SrTiO}_{3}$ substrates. Journal of the Physical Society of Japan 69, 230 (2000).

[106] S. A. Chambers. Epitaxial growth and properties of thin film oxides. Surface Science Reports 39, 105 (2000).

[107] J. Lettieri, V. Vaithyanathan, S. K. Eah, J. Stephens, V. Sih, D. D. Awschalom, J. Levy, and D. G. Schlom. Epitaxial growth and magnetic properties of EuO on (001) Si by molecular-beam epitaxy. Applied Physics Letters 83, 975 (2003).

[108] L. Maritato and A. Y. Petrov. High metal-insulator transition temperature LSMO thin films grown in low oxygen partial pressure by molecular beam epitaxy. Journal of Magnetism and Magnetic Materials 272, 1135 (2004).

[109] R. W. Ulbricht, A. Schmehl, T. Heeg, J. Schubert, and D. G. Schlom. Adsorption-controlled growth of EuO by molecular-beam epitaxy. Applied Physics Letters 93, 102105 (2008).

[110] S. Imada, S. Shouriki, E. Tokumitsu, and H. Ishiwara. Epitaxial growth of ferroelectric $\mathrm{YMnO}_{3}$ thin films on Si (111) substrates by molecular beam epitaxy. Japanese Journal of Applied Physics 37, 6497 (1998).

[111] Y. Chye, T. Liu, D. Li, K. Lee, D. Lederman, and T. H. Myers. Molecular beam epitaxy of $\mathrm{YMnO}_{3}$ on c-plane GaN. Applied Physics Letters 88, 132903 (2006).

[112] J. Kabelac, S. Ghosh, P. Dobal, and R. Katiyar. RF oxygen plasma assisted molecular beam epitaxy growth of $\mathrm{BiFeO}_{3}$ thin films on $\mathrm{SrTiO}_{3}$ (001). Journal of Vacuum Science \& Technology B 25, 1049 (2007).

[113] J. F. Ihlefeld, N. J. Podraza, Z. K. Liu, R. C. Rai, X. Xu, T. Heeg, Y. B. Chen, J. Li, R. W. Collins, J. L. Musfeldt, X. Q. Pan, J. Schubert, R. Ramesh, and D. G. Schlom. Optical band gap of $\mathrm{BiFeO}_{3}$ grown by molecular-beam epitaxy. Applied Physics Letters 92, 142908 (2008).

[114] J. A. Moyer, C. Eaton, and R. Engel-Herbert. Highly conductive SrVO $\mathrm{V}_{3}$ as a bottom electrode for functional perovskite oxides. Advanced Materials 25, 3578 (2013).

[115] I. Bozovic, J. Eckstein, and G. Virshup. Superconducting oxide multilayers and superlattices: physics, chemistry, and nanoengineering. Physica C: Superconductivity 235, 178 (1994).

[116] K. Hubbard and D. Schlom. Thermodynamic stability of binary oxides in contact with silicon. Journal of Materials Research 11, 2757 (1996). 
[117] V. Vaithyanathan, J. Lettieri, W. Tian, A. Sharan, A. Vasudevarao, Y. L. Li, A. Kochhar, H. Ma, J. Levy, P. Zschack, J. C. Woicik, L. Q. Chen, V. Gopalan, and D. G. Schlom. $\mathrm{C}$-axis oriented epitaxial $\mathrm{BaTiO}_{3}$ films on (001) Si. Journal of Applied Physics 100, 024108 (2006).

[118] R. A. McKee, F. J. Walker, and M. F. Chisholm. Physical structure and inversion charge at a semiconductor interface with a crystalline oxide. Science 293, 468 (2001).

[119] Y. Liang, J. Kulik, T. C. Eschrich, R. Droopad, Z. Yu, and P. Maniar. Hetero-epitaxy of perovskite oxides on GaAs(001) by molecular beam epitaxy. Applied Physics Letters 85, 1217 (2004).

[120] H. S. Craft, J. F. Ihlefeld, M. D. Losego, R. Collazo, Z. Sitar, and J. P. Maria. MgO epitaxy on GaN (0002) surfaces by molecular beam epitaxy. Applied Physics Letters 88, 212906 (2006).

[121] W. Tian, V. Vaithyanathan, D. G. Schlom, Q. Zhan, S. Y. Yang, Y. H. Chu, and R. Ramesh. Epitaxial integration of (0001) $\mathrm{BiFeO}_{3}$ with (0001) GaN. Applied Physics Letters 90, 172908 (2007).

[122] H. M. Smith and A. F. Turner. Vacuum deposited thin films using a ruby laser. Applied Optics 4, 147 (1965).

[123] H. Schwarz and H. A. Tourtellotte. Vacuum deposition by high-energy laser with emphasis on barium titanate films. Journal of Vacuum Science \& Technology 6, 373 (1969).

[124] J. Desserre and J. Eloy. Interaction d'un faisceau de lumière cohérente pulsée avec une cible complexe: application à l'élaboration de composés en couches minces. Thin Solid Films 29, 29 (1975).

[125] S. Zaitsev-Zotov, A. Martynyuk, and E. Protasov. Superconductivity of $\mathrm{BaPb}_{1-x} \mathrm{Bi}_{x} \mathrm{O}_{3}$ films prepared by laser evaporation method. Sovjet Physics - Solid State 25, 100 (1983).

[126] R. Groenen, J. Smit, K. Orsel, A. Vailionis, B. Bastiaens, M. Huijben, K. Boller, G. Rijnders, and G. Koster. Research Update: Stoichiometry controlled oxide thin film growth by pulsed laser deposition. APL Materials 3, 070701 (2015).

[127] H. M. Christen and G. Eres. Recent advances in pulsed-laser deposition of complex oxides. Journal of Physics: Condensed Matter 20, 264005 (2008).

[128] V. A. Shchukin and D. Bimberg. Spontaneous ordering of nanostructures on crystal surfaces. Rev. Mod. Phys. 71, 1125 (1999).

[129] W. Hong, H. N. Lee, M. Yoon, H. M. Christen, D. H. Lowndes, Z. Suo, and Z. Zhang. Persistent step-flow growth of strained films on vicinal substrates. Physical Review Letters 95, 095501 (2005).

[130] J. Tersoff, A. W. Denier van der Gon, and R. M. Tromp. Critical island size for layer-bylayer growth. Physical Review Letters 72, 266 (1994).

[131] C. M. Brooks, L. F. Kourkoutis, T. Heeg, J. Schubert, D. A. Muller, and D. G. Schlom. Growth of homoepitaxial $\mathrm{SrTiO}_{3}$ thin films by molecular-beam epitaxy. Applied Physics Letters 94, 162905 (2009). 
[132] S. Stoyanov and M. Michailov. Non-steady state effects in MBE: oscillations of the step density at the crystal surface. Surface Science 202, 109 (1988).

[133] S. Das, L. LeBrun, P. Sewell, and T. Tyrie. UHV RHEED system for in situ studies of sputtered films. Thin Solid Films 270, 314 (1995).

[134] J. Haeni, C. Theis, and D. Schlom. RHEED intensity oscillations for the stoichiometric growth of $\mathrm{SrTiO}_{3}$ thin films by reactive molecular beam epitaxy. Journal of Electroceramics 4, 385 (2000).

[135] A. Soukiassian, W. Tian, V. Vaithyanathan, J. Haeni, L. Chen, X. Xi, D. Schlom, D. Tenne, H. Sun, X. Pan, K. Choi, C. Eom, Y. Li, Q. Jia, C. Constantin, R. Feenstra, M. Bernhagen, P. Reiche, and R. Uecker. Growth of nanoscale $\mathrm{BaTiO}_{3} / \mathrm{SrTiO}_{3}$ superlattices by molecularbeam epitaxy. Journal of Materials Research 23, 1417 (2008).

[136] I. K. Robinson and D. J. Tweet. Surface X-ray diffraction. Reports on Progress in Physics 55, 599 (1992).

[137] E. Vlieg. The role of surface and interface structure in crystal growth. Progress in Crystal Growth and Characterization of Materials 62, 203 (2016).

[138] D. K. Saldin and V. L. Shneerson. Direct methods for surface crystallography. Journal of Physics: Condensed Matter 20, 304208 (2008).

[139] M. Takahasi, Y. Yoneda, H. Inoue, N. Yamamoto, and J. Mizuki. Time-resolved Xray diffraction study on surface structure and morphology during molecular-beam epitaxy growth. Journal of Crystal Growth 251, 51 (2003).

[140] M. C. Sullivan, M. J. Ward, A. Gutiérrez-Llorente, E. R. Adler, H. Joress, A. Woll, and J. D. Brock. Complex oxide growth using simultaneous in situ reflection high-energy electron diffraction and $x$-ray reflectivity: When is one layer complete? Applied Physics Letters 106, 031604 (2015).

[141] J. Ferguson. In-situ X-ray measurements during pulsed laser deposition of complex oxide materials. PhD thesis Cornell University Ithaca, NY, United States of America (August 2010).

[142] J. Z. Tischler, G. Eres, B. C. Larson, C. M. Rouleau, P. Zschack, and D. H. Lowndes. Nonequilibrium interlayer transport in pulsed laser deposition. Physical Review Letters 96, 226104 (2006).

[143] P. R. Willmott, R. Herger, C. M. Schlepütz, D. Martoccia, and B. D. Patterson. Energetic surface smoothing of complex metal-oxide thin films. Physical Review Letters 96, 176102 (2006).

[144] M. I. Larsson, W. X. Ni, and G. V. Hansson. Manipulation of nucleation by growth rate modulation. Journal of Applied Physics 78, 3792 (1995).

[145] J. D. Weeks and G. H. Gilmer. Dynamics of crystal growth. Advances in Chemical Physics 40, 344 (1979).

[146] V. S. Achutharaman, N. Chandrasekhar, O. T. Valls, and A. M. Goldman. Origin of RHEED intensity oscillations during the growth of $(Y, D y) \mathrm{Ba}_{2} \mathrm{Cu}_{3} \mathrm{O}_{7-x}$ thin films. Physical Review B: Condensed Matter and Materials Physics 50, 8122 (1994). 
[147] P. A. Maksym. Fast Monte Carlo simulation of MBE growth. Semiconductor Science and Technology 3, 594 (1988).

[148] B. Kuiper. Size effects in epitaxial oxide thin films. PhD thesis University of Twente Enschede, The Netherlands (January 2014).

[149] Q. Zhang, J. Zhu, J. Tan, G. Yu, J. Wu, J. Zhu, and D. Xiao. Monte Carlo simulation of the growth of $\mathrm{SrTiO}_{3}$ thin film with molecular source. Vacuum 81, 539 (2006).

[150] Y. Guanglong, Z. Jiangguo, Z. Jiliang, Z. Qinglei, L. Wei, T. Junzhe, and X. Dingquan. The growth simulation of $\mathrm{ABO}_{3}$ type epitaxial thin films. Integrated Ferroelectrics $\mathbf{7 8}, 85$ (2006).

[151] X. J. Zheng, B. Yang, Z. Zhu, B. Wu, and Y. L. Mao. Kinetic Monte Carlo simulation of growth of $\mathrm{BaTiO}_{3}$ thin film via pulsed laser deposition. Transactions of Nonferrous Metals Society of China 17, 1441 (2007).

[152] Z. Zhu, X. J. Zheng, and W. Li. Submonolayer growth of $\mathrm{BaTiO}_{3}$ thin film via pulsed laser deposition: a kinetic Monte Carlo simulation. Journal of Applied Physics 106, 054105 (2009).

[153] Z. Zhu, X. Zheng, and W. Li. Multilayer growth of $\mathrm{BaTiO}_{3}$ thin films via pulsed laser deposition: an energy-dependent kinetic Monte Carlo simulation. Applied Surface Science 256, 5876 (2010).

[154] S. B. Lee. Scaling in film growth by pulsed laser deposition and modulated beam deposition. Physical Review E: Statistical, Nonlinear, Biological, and Soft Matter Physics 83, 041605 (2011).

[155] F. J. Giessibl. Advances in atomic force microscopy. Reviews of Modern Physics 75, 949 (2003).

[156] Y. Seo and W. Jhe. Atomic force microscopy and spectroscopy. Reports on Progress in Physics 71, 016101 (2008).

[157] R. Garcia and R. Perez. Dynamic atomic force microscopy methods. Surface Science Reports 47, 197 (2002).

[158] A. S. Paulo and R. García. Tip-surface forces, amplitude, and energy dissipation in amplitude-modulation (tapping mode) force microscopy. Physical Review B: Condensed Matter and Materials Physics 64, 193411 (2001).

[159] L. Kuipers, M. Hoogeman, and J. Frenken. Jump to contact and neck formation between Pb surfaces and a STM tip. Surface Science 340, 231 (1995).

[160] F. Eghiaian, F. Rico, A. Colom, I. Casuso, and S. Scheuring. High-speed atomic force microscopy: imaging and force spectroscopy. FEBS Letters 588, 3631 (2014).

[161] R. C. Barrett and C. F. Quate. High-speed, large-scale imaging with the atomic force microscope. Journal of Vacuum Science \& Technology B 9, 302 (1991).

[162] T. Sulchek, R. Hsieh, J. D. Adams, S. C. Minne, C. F. Quate, and D. M. Adderton. High-speed atomic force microscopy in liquid. Review of Scientific Instruments 71, 2097 (2000). 
[163] T. Sulchek, R. Hsieh, J. D. Adams, G. G. Yaralioglu, S. C. Minne, C. F. Quate, J. P. Cleveland, A. Atalar, and D. M. Adderton. High-speed tapping mode imaging with active $Q$ control for atomic force microscopy. Applied Physics Letters 76, 1473 (2000).

[164] A. D. L. Humphris, M. J. Miles, and J. K. Hobbs. A mechanical microscope: high-speed atomic force microscopy. Applied Physics Letters 86, 034106 (2005).

[165] F. Tabak, E. Disseldorp, G. Wortel, A. Katan, M. Hesselberth, T. Oosterkamp, J. Frenken, and W. van Spengen. MEMS based fast scanning probe microscopes. Ultramicroscopy 110, 599 (2010).

[166] G. Fantner, R. Barbero, D. Gray, and A. Belcher. Kinetics of antimicrobial peptide activity measured on individual bacterial cells using high-speed atomic force microscopy. Nature Nanotechnology 5, 280 (2010).

[167] M. B. Viani, T. E. Schäffer, G. T. Paloczi, L. I. Pietrasanta, B. L. Smith, J. B. Thompson, M. Richter, M. Rief, H. E. Gaub, K. W. Plaxco, A. N. Cleland, H. G. Hansma, and P. K. Hansma. Fast imaging and fast force spectroscopy of single biopolymers with a new atomic force microscope designed for small cantilevers. Review of Scientific Instruments 70, 4300 (1999).

[168] M. Kitazawa, K. Shiotani, and A. Toda. Batch Fabrication of Sharpened Silicon Nitride Tips. Japanese Journal of Applied Physics 42, 4844 (2003).

[169] T. Ando, N. Kodera, E. Takai, D. Maruyama, K. Saito, and A. Toda. A high-speed atomic force microscope for studying biological macromolecules. Proceedings of the National Academy of Sciences 98, 12468 (2001).

[170] B. W. Hoogenboom, P. L. T. M. Frederix, J. L. Yang, S. Martin, Y. Pellmont, M. Steinacher, S. Zäch, E. Langenbach, H. J. Heimbeck, A. Engel, and H. J. Hug. A Fabry-Perot interferometer for micrometer-sized cantilevers. Applied Physics Letters $\mathbf{8 6}$, 074101 (2005).

[171] G. E. Fantner, P. Hegarty, J. H. Kindt, G. Schitter, G. A. G. Cidade, and P. K. Hansma. Data acquisition system for high speed atomic force microscopy. Review of Scientific Instruments 76, 026118 (2005).

[172] S. Hosaka, K. Etoh, A. Kikukawa, and H. Koyanagi. Megahertz silicon atomic force microscopy (AFM) cantilever and high-speed readout in AFM-based recording. Journal of Vacuum Science \& Technology B 18, 94 (2000).

[173] J. L. Yang, M. Despont, U. Drechsler, B. W. Hoogenboom, P. L. T. M. Frederix, S. Martin, A. Engel, P. Vettiger, and H. J. Hug. Miniaturized single-crystal silicon cantilevers for scanning force microscopy. Applied Physics Letters 86, 134101 (2005).

[174] T. R. Rodriguez and R. Garcia. Theory of $Q$ control in atomic force microscopy. Applied Physics Letters 82, 4821 (2003).

[175] J. Kokavecz, Z. L. Horváth, and d. Mechler. Dynamical properties of the Q-controlled atomic force microscope. Applied Physics Letters 85, 3232 (2004).

[176] H. Hölscher and U. D. Schwarz. Theory of amplitude modulation atomic force microscopy with and without Q-Control. International Journal of Non-Linear Mechanics 42, 608 (2007). 
[177] M. Jaafar, D. Martinez-Martin, M. Cuenca, J. Melcher, A. Raman, and J. Gomez-Herrero. Drive-amplitude-modulation atomic force microscopy: from vacuum to liquids. Beilstein Journal of Nanotechnology 3, 336 (2012).

[178] T. R. Albrecht, P. Grutter, D. Horne, and D. Rugar. Frequency modulation detection using high-Q cantilevers for enhanced force microscope sensitivity. Journal of Applied Physics 69, 668 (1991).

[179] T. E. Schäffer, J. P. Cleveland, F. Ohnesorge, D. A. Walters, and P. K. Hansma. Studies of vibrating atomic force microscope cantilevers in liquid. Journal of Applied Physics 80, 3622 (1996).

[180] J. Kokavecz, Z. Tóth, Z. L. Horváth, P. Heszler, and . Mechler. Novel amplitude and frequency demodulation algorithm for a virtual dynamic atomic force microscope. Nanotechnology 17, S173 (2006).

[181] K. S. Karvinen and S. O. R. Moheimani. A high-bandwidth amplitude estimation technique for dynamic mode atomic force microscopy. Review of Scientific Instruments 85, 023707 (2014).

[182] B. Schlecker, M. Ortmanns, J. Anders, and G. Fantner. PLL-based high-speed demodulation of FM signals for real-time AFM applications. (2013).

[183] G. Schitter, F. Allgöwer, and A. Stemmer. A new control strategy for high-speed atomic force microscopy. Nanotechnology 15, 108 (2004).

[184] V. Fokkema. Real-time scanning tunneling microscopy studies of thin film deposition and ion erosion. PhD thesis University of Leiden Leiden, The Netherlands (November 2011).

[185] C. T. Herbschleb, P. C. van der Tuijn, S. B. Roobol, V. Navarro, J. W. Bakker, Q. Liu, D. Stoltz, M. E. CaÃśas-Ventura, G. Verdoes, M. A. van Spronsen, M. Bergman, L. Crama, I. Taminiau, A. Ofitserov, G. J. C. van Baarle, and J. W. M. Frenken. The ReactorSTM: atomically resolved scanning tunneling microscopy under high-pressure, high-temperature catalytic reaction conditions. Review of Scientific Instruments 85, 083703 (2014).

[186] S. B. Roobol, M. E. Canas-Ventura, M. Bergman, M. A. van Spronsen, W. G. Onderwaater, P. C. van der Tuijn, R. Koehler, A. Ofitserov, G. J. C. van Baarle, and J. W. M. Frenken. The ReactorAFM: non-contact atomic force microscope operating under high-pressure and high-temperature catalytic conditions. Review of Scientific Instruments 86, 033706 (2015).

[187] N. Kodera, D. Yamamoto, R. Ishikawa, and T. Ando. Video imaging of walking myosin V by high-speed atomic force microscopy. Nature 468, 72 (2010).

[188] N. Kodera, M. Sakashita, and T. Ando. Dynamic proportional-integral-differential controller for high-speed atomic force microscopy. Review of Scientific Instruments 77, 083704 (2006).

[189] S. P. Wadikhaye, Y. K. Yong, and S. O. Reza Moheimani. A serial-kinematic nanopositioner for high-speed atomic force microscopy. Review of Scientific Instruments 85, 105104 (2014).

[190] P. K. Hansma, G. Schitter, G. E. Fantner, and C. Prater. High-speed atomic force microscopy. Science 314, 601 (2006). 
[191] A. Solmaz, M. Huijben, G. Koster, R. Egoavil, N. Gauquelin, G. Van Tendeloo, J. Verbeeck, B. Noheda, and G. Rijnders. Domain selectivity in $\mathrm{BiFeO}_{3}$ thin films by modified substrate termination. Advanced Functional Materials 26, 2882 (2016).

[192] J. D. Craggs and J. M. J. M. Meek. Electrical breakdown of gases. Chichester, New York, Wiley (1978).

[193] S. Phark and Y. Chang. Nucleation and growth of primary nanostructures in $\mathrm{SrTiO}_{3}$ homoepitaxy. Nanoscale Research Letters 10, 1 (2015).

[194] W. A. Wessels, J. J. Broekmaat, R. J. L. Beerends, G. Koster, and G. Rijnders. Fast and gentle side approach for atomic force microscopy. Review of Scientific Instruments $\mathbf{8 4}$, 123704 (2013).

[195] F. B. Segerink, J. P. Korterik, and H. L. Offerhaus. Vibration transfers to measure the performance of vibration isolated platforms on site using background noise excitation. Review of Scientific Instruments 82, 065111 (2011).

[196] B. Gotsmann and H. Fuchs. Dynamic AFM using the FM technique with constant excitation amplitude. Applied Surface Science 188, 355 (2002).

[197] J. A. Jensen, K. B. Rider, Y. Chen, M. Salmeron, and G. A. Somorjai. High pressure, high temperature scanning tunneling microscopy. Journal of Vacuum Science \& Technology B 17, 1080 (1999).

[198] M. DiBattista, S. V. Patel, J. F. Mansfield, and J. W. Schwank. In situ elevated temperature imaging of thin films with a microfabricated hot stage for scanning probe microscopes. Applied Surface Science 141, 119 (1999).

[199] M. L. Trawick, D. E. Angelescu, P. M. Chaikin, M. J. Valenti, and R. A. Register. A replaceable, low thermal mass hot stage for scanning probe microscopy. Review of Scientific Instruments 74, 1390 (2003).

[200] H. Amick, M. Gendreau, T. Busch, and C. Gordon. Evolving criteria for research facilities: vibration (2005).

[201] J. Mertens, E. Finot, T. Thundat, A. Fabre, M. Nadal, V. Eyraud, and E. Bourillot. Effects of temperature and pressure on microcantilever resonance response. Ultramicroscopy $\mathbf{9 7}$, 119 (2003).

[202] S. H. Lim, M. Murakami, J. H. Yang, S.-Y. Young, J. Hattrick-Simpers, M. Wuttig, L. G. Salamanca-Riba, and I. Takeuchi. Enhanced dielectric properties in single crystal-like $\mathrm{BiFeO}_{3}$ thin films grown by flux-mediated epitaxy. Applied Physics Letters 92, 012918 (2008).

[203] H. Béa, B. Ziegler, M. Bibes, A. Barthélémy, and P. Paruch. Nanoscale polarization switching mechanisms in multiferroic $\mathrm{BiFeO}_{3}$ thin films. Journal of Physics: Condensed Matter 23, 142201 (2011).

[204] T. Kanashima, J. M. Park, D. Ricinschi, and M. Okuyama. Columnar growth of $\mathrm{BiFeO}_{3}$ films prepared by magnetic-field-assisted pulsed laser deposition. Ferroelectrics 466, 63 (2014). 
[205] N. Deepak, P. Carolan, L. Keeney, P. F. Zhang, M. E. Pemble, and R. W. Whatmore. Bismuth self-limiting growth of ultrathin $\mathrm{BiFeO}_{3}$ films. Chemistry of Materials 27, 6508 (2015).

[206] J. Broekmaat, F. Roesthuis, D. Blank, and G. Rijnders. Side approach. European Patent EP 06076925.4 (2006).

[207] J. Fraxedas, F. Pérez-Murano, F. Gramazio, M. Lorenzoni, E. Rull Trinidad, and U. Staufer. Continuous monitoring of tip radius during atomic force microscopy imaging (2015).

[208] H. J. W. Zandvliet. Energetics of Si(001). Reviews of Modern Physics 72, 593 (2000).

[209] N. C. Bartelt, J. L. Goldberg, T. L. Einstein, and E. D. Williams. The equilibration of terrace width distributions on stepped surfaces. Surface science 273, 252 (1992).

[210] H. J. W. Zandvliet. The Ge(001) surface. Physics Reports 388, 1 (2003).

[211] K. Sotthewes and H. J. W. Zandvliet. Universal behaviour of domain wall meandering. Journal of Physics: Condensed Matter 25, 205301 (2013).

[212] H. J. W. Zandvliet, H. B. Elswijk, E. J. Loenen, and D. Dijkkamp. Equilibrium structure of monatomic steps on vicinal Si(001). Physical Review B: Condensed Matter and Materials Physics 45, 5965 (1992).

[213] P. M. Lam, S. J. Liu, and C. H. Woo. Monte Carlo simulation of pulsed laser deposition. Physical Review B: Condensed Matter and Materials Physics 66, 045408 (2002).

[214] H. J. W. Zandvliet, B. Poelsema, and H. B. Elswijk. Fluctuations of monatomic steps on Si(001). Physical Review B: Condensed Matter and Materials Physics 51, 5465 (1995).

[215] T. Nishimura, A. Ikeda, H. Namba, T. Morishita, and Y. Kido. Structure change of $\mathrm{TiO}_{2}$ terminated $\mathrm{SrTiO}_{3}(001)$ surfaces by annealing in $\mathrm{O}_{2}$ atmosphere and ultrahigh vacuum. Surface science 421, 273 (1999).

[216] J. G. Connell, B. J. Isaac, G. B. Ekanayake, D. R. Strachan, and S. S. A. Seo. Preparation of atomically flat $\mathrm{SrTiO}_{3}$ surfaces using a deionized-water leaching and thermal annealing procedure. Applied Physics Letters 101, 251607 (2012).

[217] F. Sanchez, C. Ocal, and J. Fontcuberta. Tailored surfaces of perovskite oxide substrates for conducted growth of thin films. Chemical Society Reviews 43, 2272 (2014).

[218] R. Bachelet, F. Sánchez, F. J. Palomares, C. Ocal, and J. Fontcuberta. Atomically flat SrO-terminated $\mathrm{SrTiO}_{3}$ (001) substrate. Applied Physics Letters 95, 141915 (2009).

[219] J. Canny. A computational approach to edge detection. Pattern Analysis and Machine Intelligence 8, 679 (1986).

[220] N. Nakagawa, H. Y. Hwang, and D. A. Muller. Why some interfaces cannot be sharp. Nature Materials 5, 204 (2006).

[221] C. J. Howard, B. J. Kennedy, and B. C. Chakoumakos. Neutron powder diffraction study of rhombohedral rare-earth aluminates and the rhombohedral to cubic phase transition. Journal of Physics: Condensed Matter 12, 349 (2000). 
[222] W. W. Mullins. Theory of thermal grooving. Journal of Applied Physics 28, 333 (1957).

[223] W. W. Mullins. Flattening of a nearly plane solid surface due to capillarity. Journal of Applied Physics 30, 77 (1959).

[224] W. W. Mullins. In metal surfaces: structure, energetics and kinetics. American Society for Metals, Metals Park, OH, USA (1963).

[225] A. Pimpinelli, J. Villain, D. E. Wolf, J. J. Métois, J. Heyraud, I. Elkinani, and G. Uimin. Equilibrium step dynamics on vicinal surfaces. Surface Science 295, 143 (1993).

[226] F. Wu, S. G. Jaloviar, D. E. Savage, and M. G. Lagally. Roughening of steps during homoepitaxial growth on Si(001). Physical Review Letters 71, 4190 (1993).

[227] G. S. Bales and A. Zangwill. Morphological instability of a terrace edge during step-flow growth. Physical Review B: Condensed Matter and Materials Physics 41, 5500 (1990).

[228] S. Venkatesan, B. J. Kooi, J. T. M. De Hosson, A. H. G. Vlooswijk, and B. Noheda. Substrate influence on the shape of domains in epitaxial $\mathrm{PbTiO}_{3}$ thin films. Journal of Applied Physics 102, 104105 (2007).

[229] C. E. Ekuma, M. Jarrell, J. Moreno, and D. Bagayoko. First principle electronic, structural, elastic, and optical properties of strontium titanate. AIP Advances 2, 012189 (2012).

[230] O. L. Alerhand, D. Vanderbilt, R. D. Meade, and J. D. Joannopoulos. Spontaneous formation of stress domains on crystal surfaces. Physical Review Letters 61, 1973 (1988).

[231] E. E. Gruber and W. W. Mullins. On the theory of anisotropy of crystalline surface tension. Journal of Physics and Chemistry of Solids 28, 875 (1967).

[232] G. Ballabio, M. Bernasconi, F. Pietrucci, and S. Serra. Ab initio study of yttria-stabilized cubic zirconia surfaces. Physical Review B: Condensed Matter and Materials Physics 70, 075417 (2004).

[233] S. Woo, H. Jeong, S. A. Lee, H. Seo, M. Lacotte, A. David, H. Y. Kim, W. Prellier, Y. Kim, and W. S. Choi. Surface properties of atomically flat poly-crystalline $\mathrm{SrTiO}_{3}$. Scientific reports 5, $8822(2015)$.

[234] B. Kuiper, J. L. Blok, H. J. W. Zandvliet, D. H. A. Blank, G. Rijnders, and G. Koster. Self-organization of $\mathrm{SrRuO}_{3}$ nanowires on ordered oxide surface terminations. MRS Communications 1, 17 (2011).

[235] C. Baeumer, C. Xu, F. Gunkel, N. Raab, R. A. Heinen, A. Koehl, and R. Dittmann. Surface termination conversion during $\mathrm{SrTiO}_{3}$ thin film growth revealed by $X$-ray photoelectron spectroscopy. Scientific reports 5, 11829 (2015).

[236] S. K. Hau, K. H. Wong, P. W. Chan, and C. L. Choy. Intrinsic resputtering in pulsed laser deposition of lead-zirconate-titanate thin films. Applied Physics Letters 66, 245 (1995).

[237] D. Kan, T. Shimizu, and Y. Shimakawa. Influence of cation off-stoichiometry on transport properties of metal/Nb-SrTiO 3 junctions. Journal of Applied Physics 117, 205305 (2015).

[238] H. Ning, Y. Lin, X. Hou, and L. Zhang. High thermally stable $\mathrm{BiFeO}_{3}-\mathrm{PbTiO}_{3}-\mathrm{BaTiO}_{3}$ ceramics with improved ferroelectric properties. Journal of Materials Science: Materials in Electronics 25, 1162 (2014). 
[239] A. Zakutayev, T. R. Paudel, P. F. Ndione, J. D. Perkins, S. Lany, A. Zunger, and D. S. Ginley. Cation off-stoichiometry leads to high p-type conductivity and enhanced transparency in $\mathrm{Co}_{2} \mathrm{ZnO}_{4}$ and $\mathrm{Co}_{2} \mathrm{NiO}_{4}$ thin films. Physical Review B: Condensed Matter and Materials Physics 85, 085204 (2012).

[240] S. Yilmaz, T. Venkatesan, and R. Gerhard-Multhaupt. Pulsed laser deposition of stoichiometric potassium-tantalate-niobate films from segmented evaporation targets. Applied Physics Letters 58, 2479 (1991).

[241] H. M. Christen, L. A. Boatner, J. D. Budai, M. F. Chisholm, L. A. Géa, P. J. Marrero, and D. P. Norton. The growth and properties of epitaxial $\mathrm{KNbO}_{3}$ thin films and $\mathrm{KNbO}_{3} / \mathrm{KTaO}_{3}$ superlattices. Applied Physics Letters 68, 1488 (1996).

[242] J. Smit. On the sticking of species; investigation of the off-stoichiometry caused by processes during PLD growth of perovskites containing volatile species. Master's thesis University of Twente Enschede, The Netherlands (June 2015).

[243] Z. Dai, A. Miyashita, S. Yamamoto, K. Narumi, and H. Naramoto. Crystalline and nearly stoichiometric vanadium nitride thin film by PLD. Thin Solid Films 347, 117 (1999).

[244] T. Michely and J. Krug. Islands, mounds and atoms: patterns and processes in crystal growth far from equilibrium. Springer-Verlag Berlin Heidelberg (2004). 



\section{Summary}

Atomic force microscopy (AFM) belongs to the selection of scanning probe microscopes (SPM). Since its invention in 1986, AFM has been applied for studying surfaces under a variety of conditions. The principle of the microscope is based on the interaction force measured between AFM tip and sample surface. While rasterscanning the AFM tip over the sample surface, the interaction force (and therefore distance) between AFM tip and sample surface is kept constant by using a feedback loop (in constant force mode). By acquiring the (X, Y and $\mathrm{Z}$ ) piezo positions, this data can be translated into a topographical image. Conventional AFM's have low sample throughput determined by the bandwidths (which are fairly low) of the AFM scanner, cantilever, detection system and electronics. However, in order to monitor the oxide pulsed laser deposition (PLD) growth kinetics, an increase in AFM bandwidth is required. Throughout this thesis, an AFM design, containing a high resonance frequency AFM scanner together with high bandwidth SPM electronics is demonstrated, with focus on growth monitoring during PLD of oxides. Moreover, perovskite oxide growth (related) studies are explored using ex situ AFM and reflection high-energy electron diffraction (RHEED).

The majority of this work is focused on the developed AFM. This AFM contains a flexure scanner, typically used in the field of high speed AFM, and high bandwidth SPM electronics. The AFM is capable of operating under conditions ranging from pressures $10^{-6}-1$ mbar $\mathrm{O}_{2}$ at temperatures ranging from $\mathrm{RT}$ up to $700{ }^{\circ} \mathrm{C}$. A proof of principle is shown by imaging of the growth of $\mathrm{BiFeO}_{3}$ islands on a $\mathrm{SrTiO}_{3}$ substrate under PLD conditions with (sub)nanometer height resolution. Furthermore, neck formation is limiting imaging of mixed terminated $\mathrm{SrTiO}_{3}$ using a Si AFM tip. Now the AFM acquisition rate under oxide PLD conditions is limited by the large response time of the cantilever. The cantilever response time can be lowered by increasing both, the damping and resonance frequency of the cantilever. Moreover, an increase in bandwidth of the detection system is needed for quasi real-time growth observations. 
In order to quasi real-time monitor the oxide growth kinetics, a tip-sample (side) approach method using a faster transfer stage has been demonstrated. This tipsample approach method, reducing the tip-sample approach time, can be applied when deposition and growth are separated in time. Both transfer stage motion time and reposition repeatability are important for monitoring PLD growth of islands using AFM. The experimental results reveal that the transfer stage motion time between the PLD and AFM position can be reduced from several seconds to (sub)seconds with a reposition repeatability error of $\pm 60 \mathrm{~nm}$. Moreover, no signatures of loss in AFM spatial resolution are found even after more than hundred repetitive side approaches.

Diagnostic tools, such as RHEED can be utilized to extract the activation energy barrier for diffusion however, it does not allow extracting the nearest neighbor interaction energy $E_{\mathrm{N}}$. The last is an important parameter for describing perovskite oxide thin film growth by kinetic Monte Carlo (KMC) simulations. The $E_{\mathrm{N}}$ values reported in literature however, show a wide spread. In this thesis, perovskite oxide vicinal surfaces in thermodynamic equilibrium are analyzed, analogue to earlier introduced analysis performed on STM images of semiconductor surfaces, after imaging ex situ AFM topography images. From perovskite oxide surfaces in thermodynamic equilibrium, the nearest neighbor interaction energy is extracted from AFM image analysis, which is impossible to extract with reciprocal diagnostic tools. The nearest neighbor interaction energy for $\operatorname{SrTiO}_{3}(001)$ and $\mathrm{DyScO}_{3}(110)$ is determined. This value is consistent with reported literature values. Most likely, this value is found to be constant for perovskite oxides due to the similar local oxygen coordination.

Furthermore, a study has been presented investigating growth of perovskite oxides and sticking of volatile species. Both KMC simulations and experiments of $\mathrm{SrTiO}_{3}$ homoepitaxial growth reveal that the amplitude of RHEED specular intensity oscillations can be increased using temperature modulation pulsed laser deposition (TM-PLD). The increase of RHEED specular intensity oscillations is explained by the fact that the step density modulation during $2 \mathrm{D}$ growth is increased using a TM-PLD sequence, where approximately the first half of the monolayer (ML) is grown at lower $T$, while the second half of the ML is grown at higher $T$. An increased $\mathrm{Pb}$ :Ti ratio is found for $\mathrm{PbTiO}_{3}$ films grown using TM-PLD compared to deposition at constant temperature. TM-PLD can be utilized most likely to enhance sticking of volatile elements during PLD growth. More research is needed in order to relate the increased $\mathrm{Pb}$ :Ti ratio to the increased step density during growth. 
In the case of PLD, the development of an AFM suitable for monitoring growth by the use of e.g. the side approach together with (relevant) AFM growth studies, demonstrating the need for quasi real-time measurement of local perovskite oxide growth are presented. A high resonance frequency AFM scanner and high bandwidth SPM electronics are developed. Some efforts are recommended increasing the bandwidth of both the cantilever and (optical) detection system in order to increase the maximum achievable AFM acquisition rate under oxide PLD conditions. This is required for enabling AFM monitoring of growing perovskite oxide islands during PLD. Moreover, increasing both the AFM feedback bandwidth and transfer stage speed will result in a faster tip-sample approach time using the side approach method. 



\section{Samenvatting}

Atomic force microscopy (AFM) behoort tot de groep van scanning probe microscopes (SPM). Sinds de uitvinding van AFM in 1986 is de microscoop techniek toegepast om oppervlakken te bestuderen onder diverse omstandigheden. Het werkingsprincipe is gebaseerd op een wisselwerkingskracht tussen de AFM naald en monsteroppervlak. Tijdens het rasterscannen van de AFM naald over het monsteroppervlak wordt de wisselwerkingskracht constant gehouden door gebruik te maken van een terugkoppelingslus. Doordat de X, Y, Z posities worden opgenomen kan een topografisch plaatje worden gereconstrueerd. Gebruikelijke AFM's hebben een lage data verwerkingscapaciteit die op zijn beurt door de bandbreedte van de AFM scankop, bladveer, detectiesysteem en elektronica wordt bepaald. Echter, een toename van AFM bandbreedte is nodig om in staat te zijn om de groei kinetische processen tijdens gepulste laser depositie (PLD) te volgen. In dit proefschrift, een AFM ontwerp, bestaande uit een hoge resonantie frequentie AFM scankop en elektronica, is gedemonstreerd met doel de groei van oxidische materialen met behulp van PLD te volgen. Daarnaast zijn perovskiet oxidische gerelateerde groei studies beschreven die zijn onderzocht door gebruik te maken van technieken zoals ex situ AFM en reflectie hoge energie electronendiffractie (RHEED).

Het grootste deel van het werk is gericht op de ontwikkelde AFM. Deze AFM bevat een hoge bandbreedte AFM elektronica en een flexure scankop die typisch gebruikt wordt in hoge snelheids AFM toepassingen. De microscoop is in staat om afbeeldingen te maken van oxidische oppervlakken op temperaturen van kamer temperatuur $\mathrm{t} / \mathrm{m} 700{ }^{\circ} \mathrm{C}$ in drukken varierend van $10^{-6} \mathrm{t} / \mathrm{m} 1 \mathrm{mbar} \mathrm{O}_{2}$. Dit principe is bewezen door (sub) nanometer resolutie afbeeldingen op te nemen van $\mathrm{BiFeO}_{3}$ eilanden op een $\mathrm{SrTiO}_{3}(001)$ monster in PLD condities. Daarnaast is geobserveerd dat nek formatie de oorzaak is dat dubbel getermineerde oppervlakken niet kunnen worden afgebeeld wanneer gebruik wordt gemaakt van een Si AFM naald. Op dit moment is de AFM snelheid in oxidische PLD condities beperkt door de lange reactietijd van de bladveer. De bladveer reactietijd kan worden verkort door zowel de demping en resonantie frequentie te verhogen. Daarnaast 
zou de detectie bandbreedte moeten worden verhoogd om quasi real-time groei observaties te bewerkstelligen.

Een laterale naald-monster naderingsmethode is gedemonstreerd door gebruik te maken van een snellere verplaatsings mechanisme om oxidische groei kinetische processen te volgen. Deze naald-monster naderingsmethode, met een veel kortere naderingstijd, kan toegepast worden wanneer depositie en groei zijn gescheiden in tijd. Zowel de monster verplaatsingstijd als repositioneringsherhaaldelijkheid zijn belangrijk voor het volgen van groeiende PLD gedeponeerde eilanden met AFM. De experimentele resultaten onthullen dat de naderingstijd kan worden verkort van enkele seconden tot subseconden met een repositioneringsherhaaldelijkheid van $\pm 60 \mathrm{~nm}$. Verder zijn er geen sporen gevonden van een verlies in AFM resolutie zelfs niet na meer dan honderd herhaaldelijke laterale naderingen.

Diagnostische technieken zoals RHEED kunnen worden gebruikt voor het bepalen van de activatie barriere voor diffusie, maar niet voor het bepalen van de naaste nabuur energie $E_{\mathrm{N}}$. De laatste is een belangrijke parameter voor het beschrijven van perovskiet oxidische dunne film groei met behulp van kinetische Monte Carlo (KMC) simulaties. Echter, de $E_{\mathrm{N}}$ waardes beschreven in literatuur vertonen een grote spreiding. In dit proefschrift zijn perovskiet oxidische oppervlakken in thermodynamisch evenwicht geanalyseerd vergelijkbaar met een eerder geintroduceerde analyse toegepast op halfgeleider oppervlakken, nadat oppervlakte afbeeldingen zijn gemaakt met ex situ AFM. Het bepalen van $E_{\mathrm{N}}$ is onmogelijk met reciproke technieken zoals RHEED. De $E_{\mathrm{N}}$ waardes zijn bepaald voor zowel het $\mathrm{SrTiO}_{3}(001)$ als $\mathrm{DyScO}_{3}(110)$ oppervlak. De gevonden waardes zijn vrijwel vergelijkbaar met de waardes beschreven in literatuur. Zeer waarschijnlijk is deze waarde constant voor alle perovskiet oxidische materialen met dezelfde vergelijkbare oxidische coordinatie aan het oppervlak.

Daarnaast wordt er een studie gepresenteerd waarin de groei en sticking van (vluchtige) perovskiet oxidische materialen zijn bestudeerd. Zowel KMC simulaties als $\mathrm{SrTiO}_{3}$ homoepitaxiale groei experimenten onthullen dat de amplitude van RHEED intensiteits oscillaties kunnen worden vergroot door gebruik te maken van temperatuur gemoduleerde gepulste laser depositie (TM-PLD). De toename in amplitude van de RHEED intensiteits oscillaties is verklaard door het feit dat de stap dichtheid modulatie is toegenomen tijdens 2D groei en een TM-PLD volgorde waarbij de eerste helft van de monolaag is gegroeid op een lagere temperatuur en de tweede helft op hogere temperatuur. Een toegenomen $\mathrm{Pb}: \mathrm{Ti}$ verhouding is gevonden voor $\mathrm{PbTiO}_{3}$ dunne lagen die gegroeid zijn met TM-PLD wanneer ze vergeleken worden met depositie op constante temperatuur. TM-PLD kan hoogstwaarschijnlijk worden gebruikt om de sticking van vluchtige elementen in 
perovskiet oxidische PLD groei te verhogen. Meer onderzoek is nodig om te bewijzen dat de toegenomen $\mathrm{Pb}$ :Ti verhouding is gerelateerd aan de toegenomen stap dichtheid tijdens de groei.

De ontwikkeling van een AFM geschikt voor de volgen van groei met behulp van de laterale naald-monster naderingsmethode en AFM gerelateerde groei studies in het geval van PLD zijn gepresenteerd. Dit werk demonstreert de noodzaak om quasi real-time metingen te kunnen doen aan lokale perovskiet oxidische groei. Zowel een hoge resonantie frequentie scankop als hoge bandbreedte AFM elektronica zijn ontwikkeld in dit werk. Er worden werkzaamheden aangeraden om de maximale haalbare AFM acquisitie snelheid in PLD condities te verhogen door zowel de bandbreedte van de bladveer als detectiesysteem te verhogen. Dit is noodzakelijk om de lokale groei van perovskiet oxidische eilanden te kunnen volgen met AFM. Daarnaast zou de laterale naald-monster naderingsmethode snelheid kunnen worden verhoogd door de AFM terugskoppelingslus bandbreedte en monster verplaatsingssnelheid te verhogen. 



\section{Publications}

- W.A. Wessels, G. Koster \& G. Rijnders, Sticking of volatile species in perovskite oxides, in preparation.

- W.A. Wessels, G. Koster, H.J.W. Zandvliet \& G. Rijnders, Energetics of vicinal perovskite oxide surfaces, under review.

- W.A. Wessels, T.R.J. Bollmann, G. Koster \& G. Rijnders, Real-time afbeelden van dunne film groei, NEVACBLAD 53, 6 (2015).

- W.A. Wessels, J.J. Broekmaat, R.J.L. Beerends, G. Koster \& G. Rijnders. Fast and gentle side approach for atomic force microscopy, Review of Scientific Instruments 84, 123704 (2013).

- M. Bayraktar, W.A. Wessels, C.J. Lee, F.A. van Goor, G. Koster, G. Rijnders \& F. Bijkerk, Active multilayer mirrors for reflectance tuning at extreme ultraviolet (EUV) wavelengths, Journal of Physics D: Applied Physics 45, 494001 (2012). 



\section{Curriculum Vitae}

Werner Wessels was born in Markelo on December 4, in 1986. He started to study Applied Physics at the Saxion Hogeschool Enschede in 2005. In 2009, he received a B. Eng. degree in Applied Physics. Thereafter, he continued to study Applied Physics at the University of Twente. In 2012, he received a M.Sc. degree in Applied Physics from the University of Twente Enschede, The Netherlands. After obtaining a M.Sc. degree, Werner accepted a Ph.D. position in the Inorganic Materials Science group at the University of Twente under supervision of Prof. dr. ing. Guus Rijnders and Prof. dr. ir. Gertjan Koster. Werner has continued his career as R\&D engineer at Twentsche Kabelfabriek in Lochem, The Netherlands. 



\section{Dankwoord}

Aangekomen bij het laatste (en meest gelezen) hoofdstuk, besef ik me dat dit niet in enkele minuten kan worden geschreven. De reden is dat ondanks dat ik het volledige proefschrift heb geschreven, de totstandkoming niet mogelijk was zonder de hulp en inspiratie van andere mensen. Daarnaast hebben diverse mensen mij geholpen en de mogelijkheid geboden om überhaupt aan een promotietraject te beginnen. Terugkijkend concludeer ik dat ik voor het eerst in contact kwam met de vakgroep Inorganic Materials Science (IMS) in het jaar 2009, het jaar waarin ik begon aan mijn HBO afstudeerstage. De laatste vier jaar heb ik besteed aan een "uitdagend" promotieonderzoek. In de zeven jaar bij IMS heb ik vele leuke mensen leren kennen binnen en buiten deze vakgroep.

Allereerst wil ik mijn begeleiders bedanken. Guus, bedankt voor je vertrouwen om mij een "hands on" promotieproject als dit te laten uitvoeren. Jouw ondersteuning, kritische houding en enthousiasme heb ik vanaf dag één zeer gewaardeerd. Daarnaast heb je me veel vrijheid gegeven om het onderzoek zelfstandig uit te voeren. Vervolgens wil ik mijn dagelijkse begeleider Gertjan (Koster) bedanken voor zijn idëeen, discussies en de correcties op het schrijfwerk. Jouw textuele correcties zorgden ervoor dat geschreven teksten toch wat fijner lezen. Ik wens jullie beiden veel succes in de toekomst.

Joska, jij hebt eraan bijgedragen dat het project van de grond kwam. Samen reden we op en neer naar Philips om de experimenten van hoofdstuk 4 uit te voeren en naar Leiden voor de bezoeken aan Leiden Probe Microscopy BV. Onze gesprekken waren voor mij leerzaam en gezellig. Bij deze bedankt voor jouw enthousiasme om mij te helpen. Tjeerd wil ik bedanken voor de samenwerking en hulp in de tweede helft van mijn promotieonderzoek. De discussies en de correcties met betrekking tot mijn proefschrift hebben gezorgd dat er uiteindelijk een "boekje" is gekomen. Daarnaast wil ik Dominic in het bijzonder bedanken. Zonder jou was er geen vacuüm setup geweest en had het project niet uitgevoerd kunnen worden. Mede doordat we dezelfde humor hebben was het ook prettig samenwerken! 
Hoofdstuk 3 is de beschrijving van de ontwikkelde opstelling waaraan de mensen van Leiden Probe Microscopy BV een hele grote bijdrage aan hebben geleverd. Veel dank ben ik verschuldigd aan Gertjan (van Baarle) en Alexei. In deze samenwerking hebben we de AFM ontwikkeld die beschreven staat in hoofdstuk 3. Daarnaast heb ik jullie gastvrijheid zeer gewaardeerd. Gertjan stond mij bij elk telefoontje te woord! Gertjan, ik wens je veel succes met jouw bedrijf en dank je voor het plaatsnemen in mijn commissie. Hoofdstuk 4 beschrijft de side approach experimenten die zijn uitgevoerd bij Philips Miplaza. Patrick Markus en Rene Beerends wil ik bedanken voor het mogelijk maken om de AFM bij Philips te gebruiken voor onze experimenten. Ksenia, thank you for the fabricated nanocross samples. Voor de samenwerking met betrekking tot enkele experimenten uit Hoofdstuk 6 wil ik Rik noemen in dit dankwoord.

Hoofdstuk 5 is ontstaan uit een hele fijne samenwerking met Harold Zandvliet. Je was altijd bereid om te discussiëren, te corrigeren (binnen een dag nagekeken) en ook in voor praatjes over zaken buiten de wetenschap. Veel dank voor dit alles, voor het plaatsnemen in mijn Ph.D. commissie en veel succes in de toekomst met het leiden van de PIN groep. Ook wil ik de andere commissieleden Prof. dr. ir. B.J. Kooi en Prof. dr. J. Aarts bedanken voor de moeite die zij hebben genomen. Cowboy projecten, zoals hetgene beschreven in dit proefschrift, zijn (nagenoeg) niet mogelijk op de UT zonder Dave. Ook jou wil ik bedanken voor jouw interesse en het plaatsnemen in mijn commissie.

I want to thank the people with who I shared the office: Michelle, Boota, Nirupam, Alim and Jun. Henk Veldhuis is inmiddels met pensioen, maar ik wil je bedanken voor jouw hulp in verschillende fases bij IMS waardoor ik weer verder kon. Daarnaast namen we de "stand van zaken" door wanneer we als eerste in de koffieruimte waren. Frank, Dick, Karin en Laura stonden ook altijd klaar voor de technische ondersteuning. Al weet ik niet of Frank altijd blij met mij was omdat ik de multimeter van het sputterlab ruim een jaar heb "geleend". Marion, bedankt voor alle administratieve zaken die je voor mij geregeld hebt. Je zorgde ervoor dat alles zo spoedig mogelijk geregeld was! Door José heb ik de nodige apparatuur en/of spullen kunnen bestellen waardoor ik de ruimte had om het "één en ander" te testen. Martin Siekman, Frans Segerink en Mark Smithers wil ik noemen aangezien zij altijd klaar stonden om mij zo snel mogelijk te helpen. Zo kreeg ik dus hulp van meerdere technici uit andere groepen!

Daarnaast wil ik de huidige IMS leden Bernard, André, Evert, Mark, Kees, Lin, Zhaoliang, Minh Nguyen, Deepak, Ruud, Ben, Huiyu, Gerard, Daniel, Minh Thanh Do, Petra, David, Jaap, Ron, Jun bedanken als mede de oud IMS leden Anirban, Kurt, Pablo, Rik, Alim, Sjoerd, Michelle, Maarten, Nirupam, Peter 
Brinks, Brian, Rogier, Bouwe, Wouter, Xin, Josée, Peter de Veen. De studietour met IMS naar Oxford was erg gezellig. Op conferenties en uitjes, waaronder de MRS Boston en de workshop in Ameland, heb ik me kostelijk vermaakt met Tom, Kai, Anirban, Kurt en anderen.

Kai en Tom, ik wil jullie verder nog bedanken voor jullie ondersteuning bij de verdediging (paranimfen) en voor alle "dram" die we gehad hebben. Samen begonnen als HBO studenten in 2005 en nu allemaal minimaal in de laatste fase van het promotieonderzoek. Kai, met jou was het altijd fijn samenwerken aangezien ik wist waar ik aan toe was en we het beste in elkaar naar boven haalden. In combinatie met het nodige "ouwehoeren" werd het resultaat er niet minder om. Ondanks dat we de promotie niet in dezelfde vakgroep uitoefenden bleven we samen pauzes houden. In deze pauzes werden allerlei discussies gevoerd over onze promoties, sport, bier en waarover eigenlijk niet. Ik wil jou veel succes wensen met jouw verdere loopbaan in de wetenschap en veel geluk met Shirley en Felien. Tom, jouw leercurve in de "dram" is ongekend maar maakte het er niet minder gezellig op. $\mathrm{Nu}$ hoop ik wel dat je een keer gaat beginnen aan het schrijven van jouw thesis/draft, want ik heb wel zin in jouw promotiefeestje! Kortom, ik wens jou ook veel succes in de toekomst met wat je doet en gaat doen. Buiten het werk om wil ik mijn vrienden bedanken, in het bijzonder Leon. Er zaten herkenningspunten in onze promotieonderzoeken, hoewel jij het onderzoek uitvoerde in een ander veld. Bedankt voor de gesprekken en discussies.

Bijzonder dankbaar ben ik voor de steun van mijn ouders en mijn (schoon)familie. Mijn ouders (Jan en Giny) hebben mij altijd gesteund en gestimuleerd om wat te bereiken in het leven. Zij hebben mij bijgebracht om gedisciplineerd te werken zowel op het werk, sport en op ander gebied. Ik wil mijn zus(je) en broer (en aanhang/kinderen) bedanken voor de steun. Er zijn raakvlakken, ondanks dat we alle drie op totaal verschillende vakgebieden aktief zijn. Ik wil mijn schoonfamilie bedanken voor hun gastvrijheid en de gezelligheid. Tot slot wil ik mijn vrouw Marije bedanken. Jouw liefde heeft ervoor gezorgd dat ik thuiskwam als "hotelgast", tijd had voor het schrijven van het proefschrift en voor andere dingen. Ik weet van binnen dat jij zo hebt verlangd naar het moment dat het proefschrift geschreven is. Het is zover! Mede dankzij jouw geduld ben ik zover gekomen. Ik heb zin in onze toekomst samen met onze pasgeboren dochter Nora!

Werner Wessels, Enschede, December 2016. 


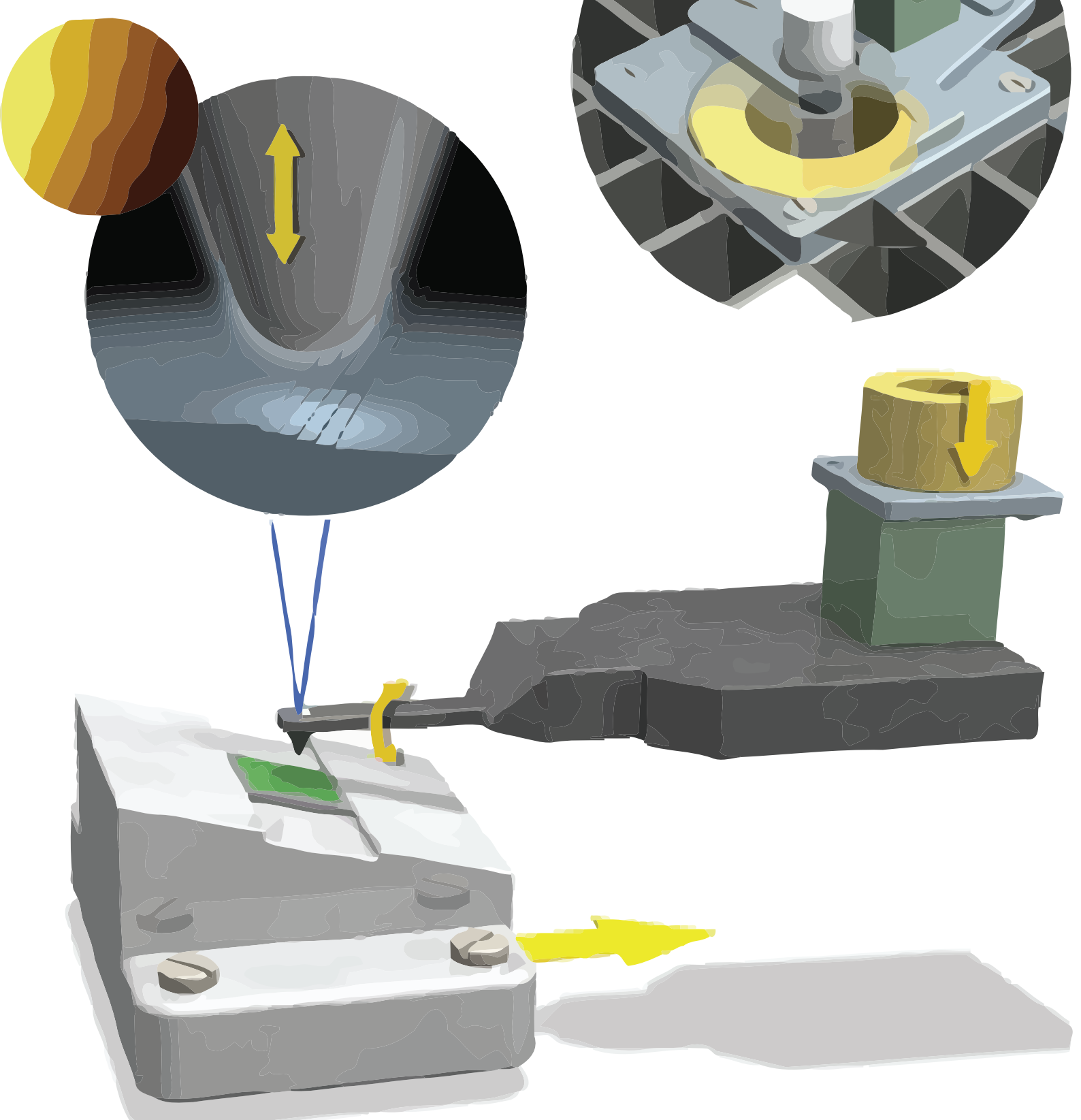

ISBN: 978-90-365-4219-7 